Review

\title{
Human matrix metalloproteinases: An ubiquitarian class of enzymes involved in several pathological processes
}

\author{
Diego Sbardella, Giovanni Francesco Fasciglione, Magda Gioia, Chiara Ciaccio, \\ Grazia Raffaella Tundo, Stefano Marini*, Massimo Coletta* \\ Department of Experimental Medicine and Biochemical Sciences, University of Roma Tor Vergata, Via Montpellier 1, I-00133 Roma, Italy \\ Interuniversity Consortium for the Research on the Chemistry of Metals in Biological Systems, Via C. Ulpiani 27, I-70126 Bari, Italy
}

\section{A R T I C L E I N F O}

\section{Article history:}

Available online 10 November 2011

\section{Keywords:}

Matrix metalloproteinases

Endopeptidases

Structure

Function

Pathological role

Cellular compartmentalization

\begin{abstract}
A B S T R A C T
Human matrix metalloproteinases (MMPs) belong to the M10 family of the MA clan of endopeptidases. They are ubiquitarian enzymes, structurally characterized by an active site where a $\mathrm{Zn}^{2+}$ atom, coordinated by three histidines, plays the catalytic role, assisted by a glutamic acid as a general base. Various MMPs display different domain composition, which is very important for macromolecular substrates recognition. Substrate specificity is very different among MMPs, being often associated to their cellular compartmentalization and/or cellular type where they are expressed. An extensive review of the different MMPs structural and functional features is integrated with their pathological role in several types of diseases, spanning from cancer to cardiovascular diseases and to neurodegeneration. It emerges a very complex and crucial role played by these enzymes in many physiological and pathological processes.
\end{abstract}

(c) 2011 Elsevier Ltd. All rights reserved.

\section{Contents}

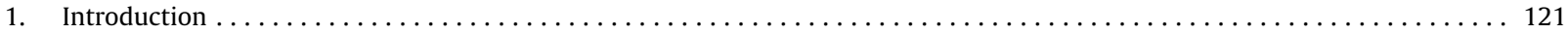

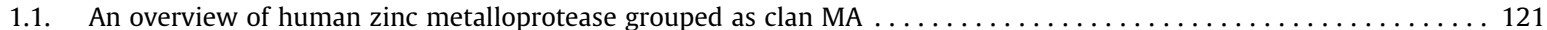

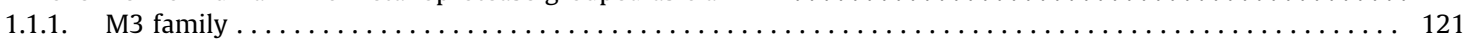

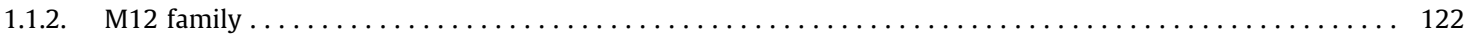

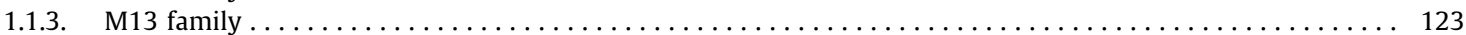

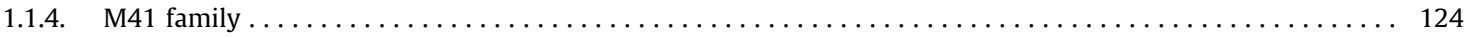

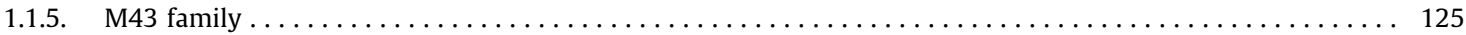

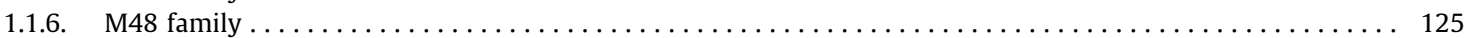

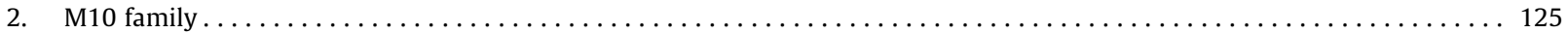

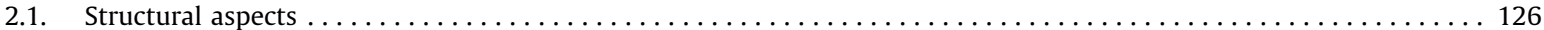

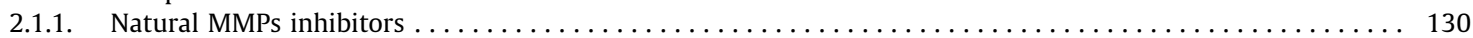

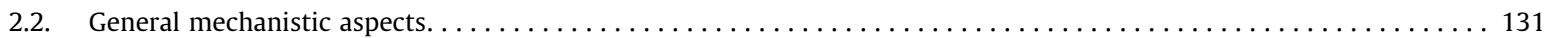

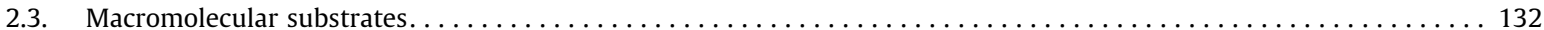

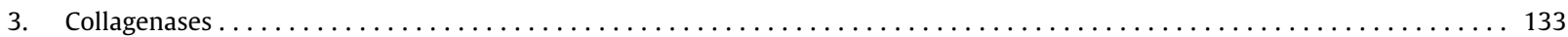

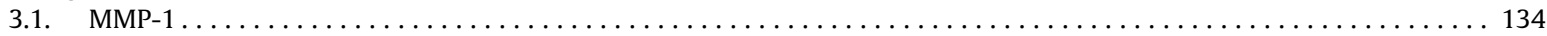

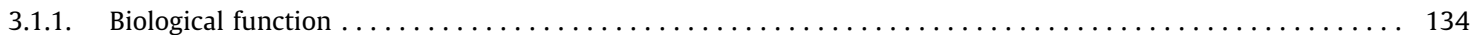

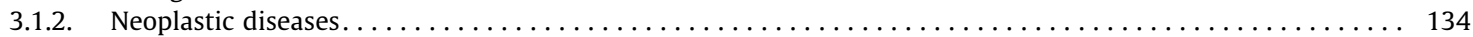

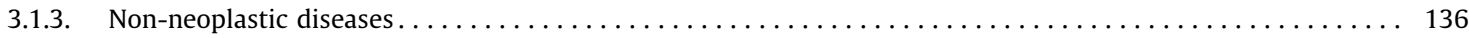

\footnotetext{
* Corresponding authors at: Department of Experimental Medicine and Biochemical Sciences, University of Roma Tor Vergata, Via Montpellier 1, I-00133 Roma, Italy. Tel.: +39 06 72596354; fax: +39 0672596353 (S. Marini), tel.: +39 06 72596365; fax: +39 0672596353 (M. Coletta).

E-mail addresses: stefano.marini@med.uniroma2.it (S. Marini), coletta@seneca.uniroma2.it, coletta@uniroma2.it (M. Coletta).
} 


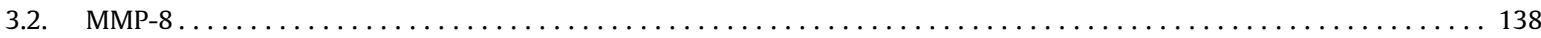

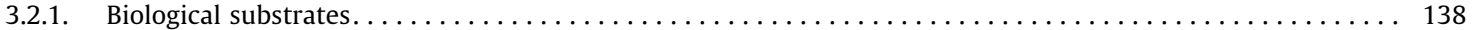

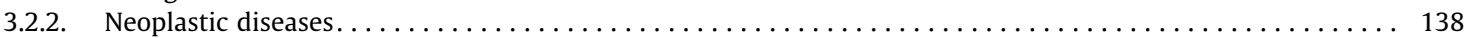

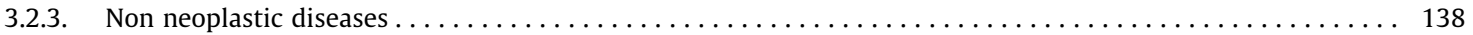

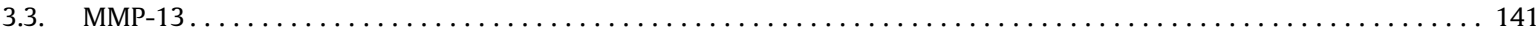

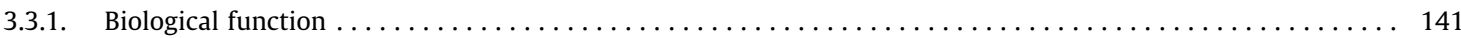

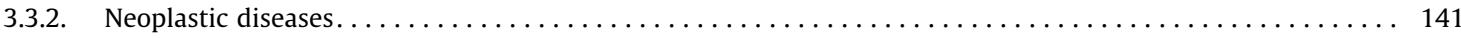

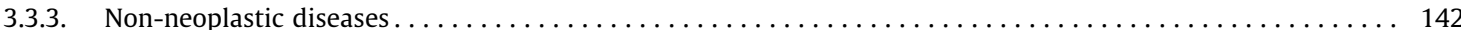

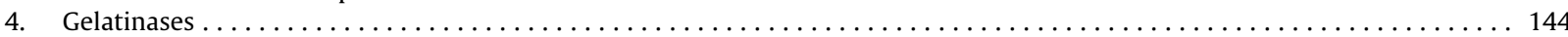

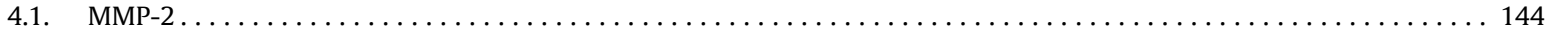

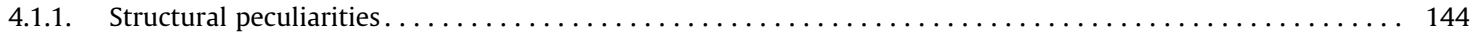

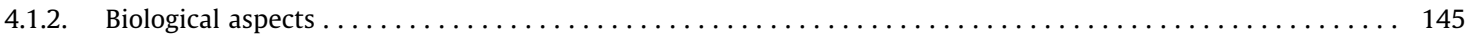

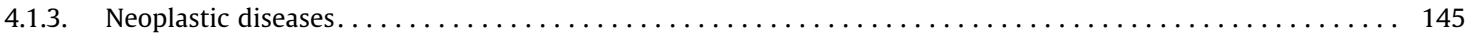

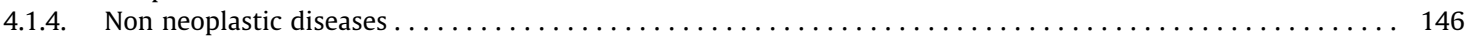

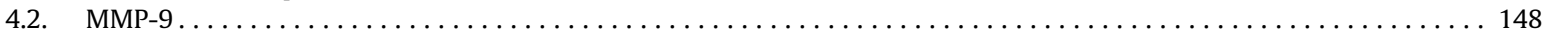

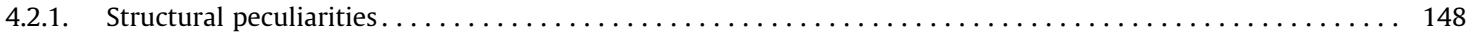

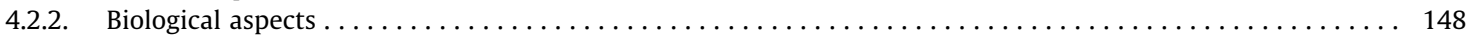

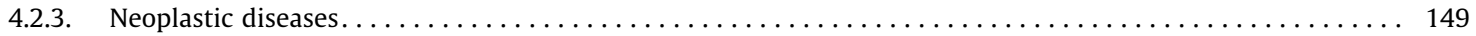

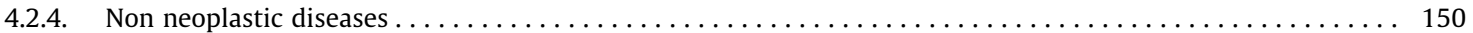

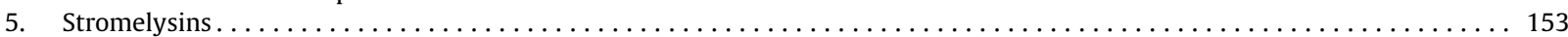

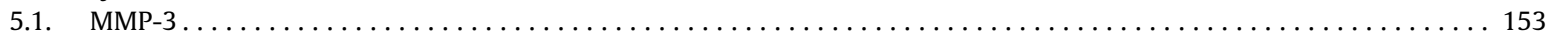

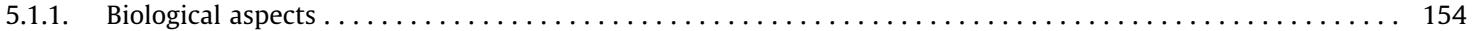

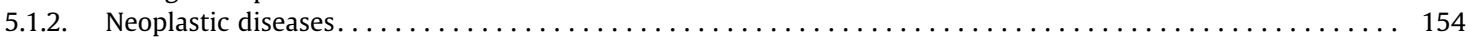

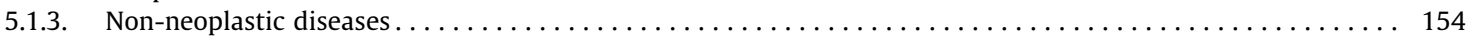

5.2. $\quad$ MMP-10 . . .

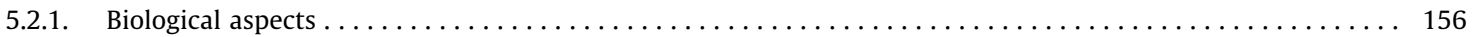

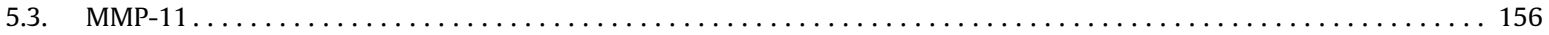

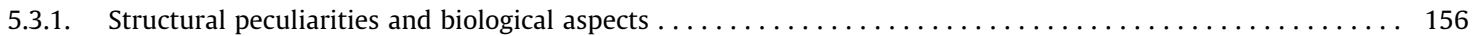

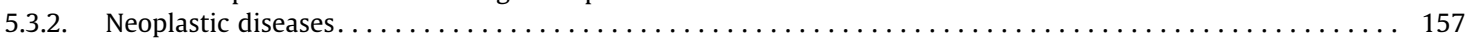

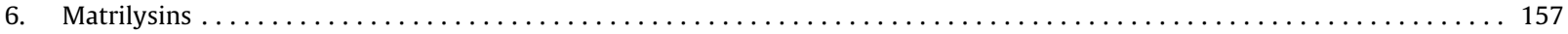

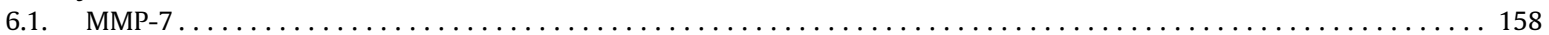

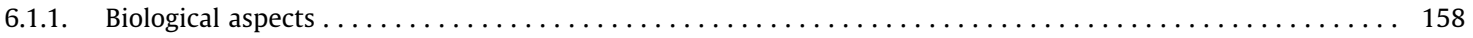

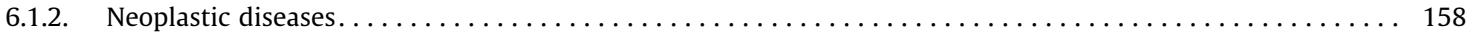

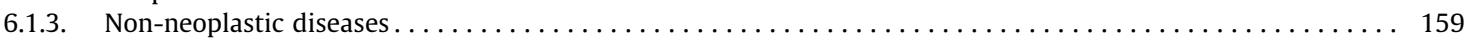

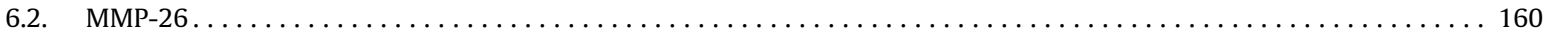

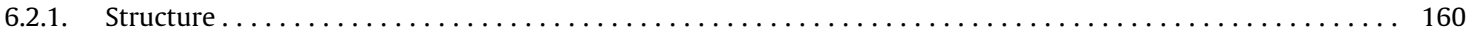

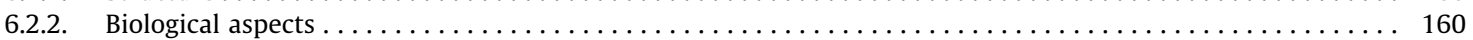

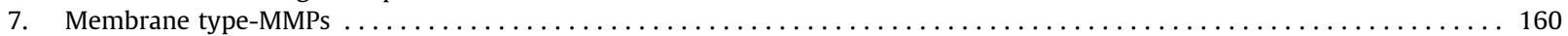

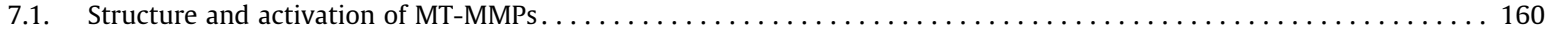

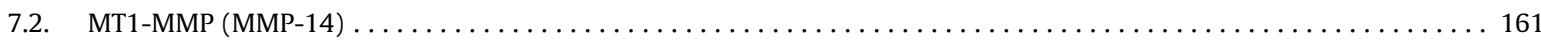

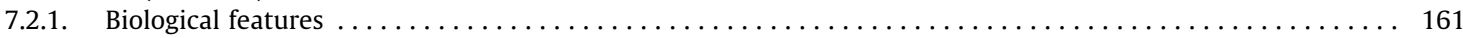

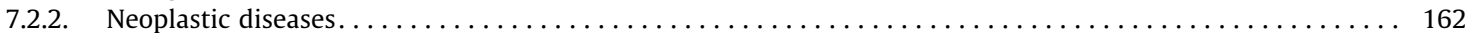

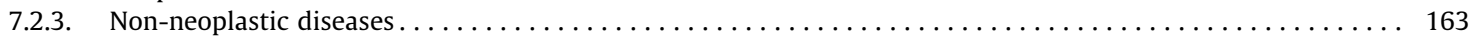

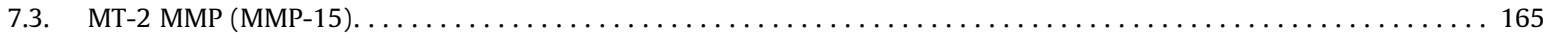

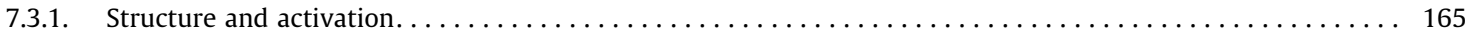

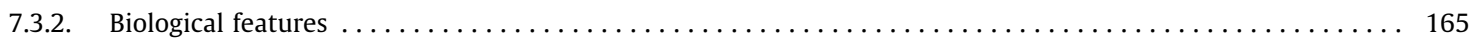

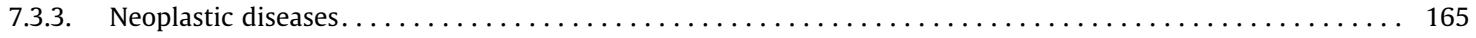

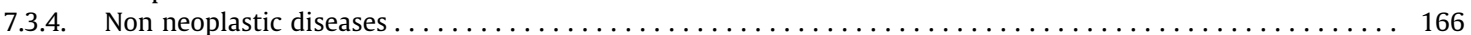

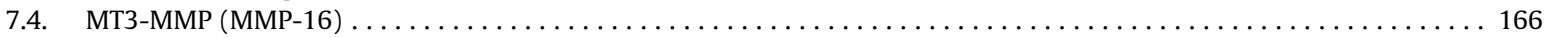

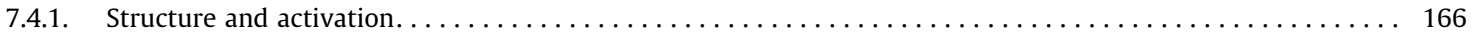

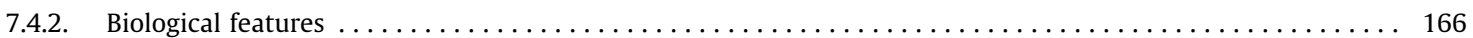

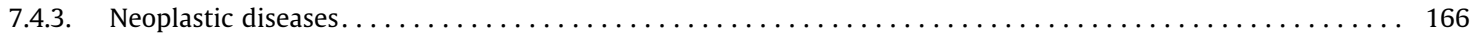

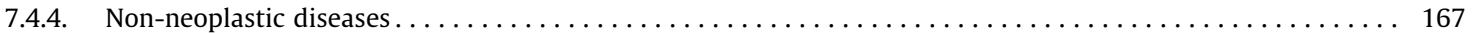

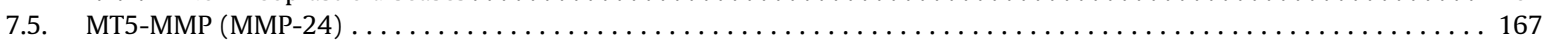

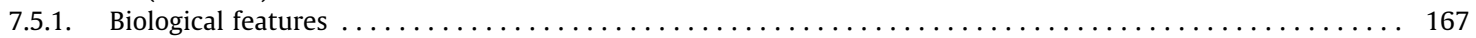

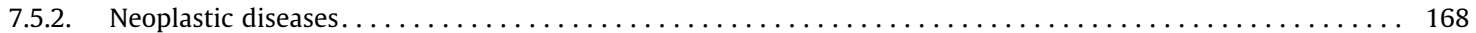

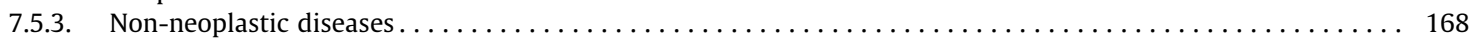

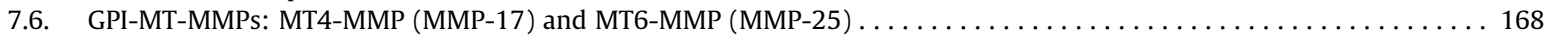

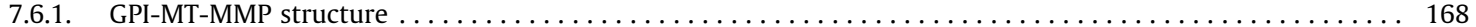

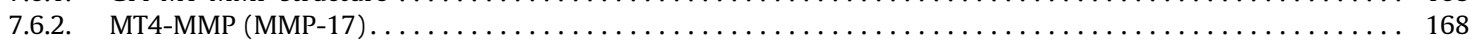

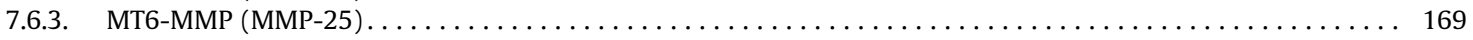

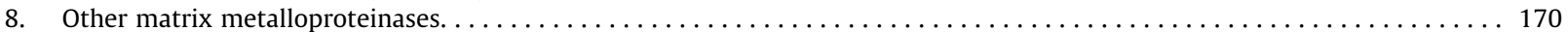

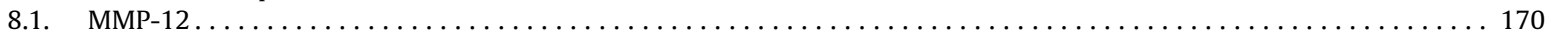

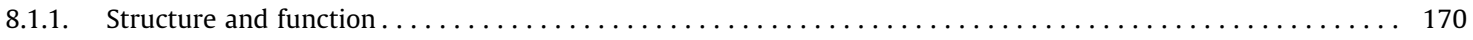

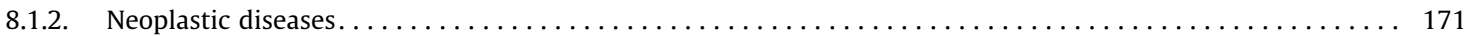

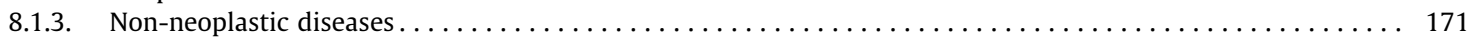




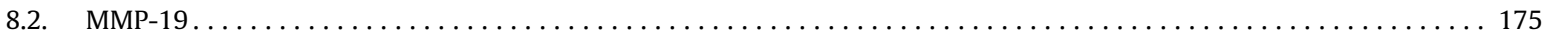

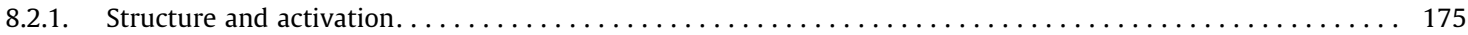

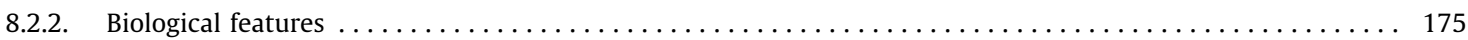

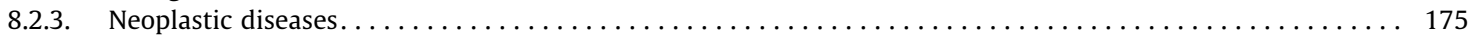

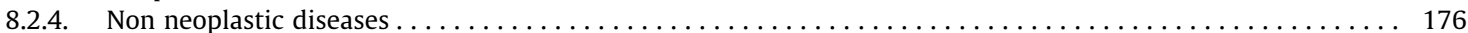

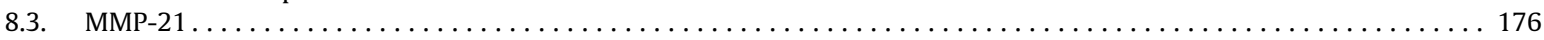

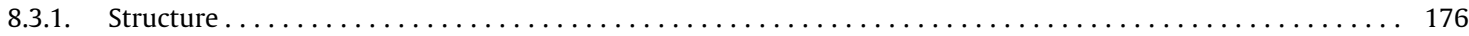

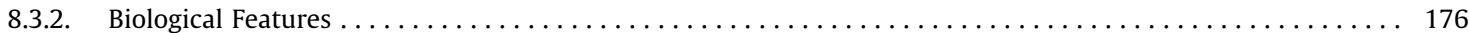

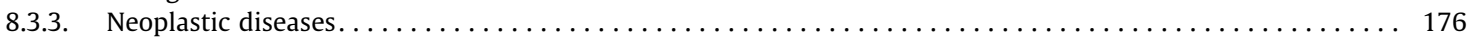

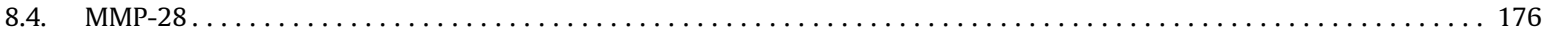

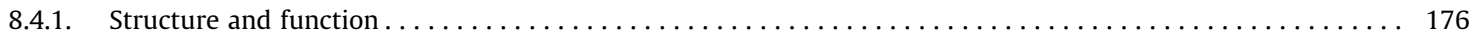

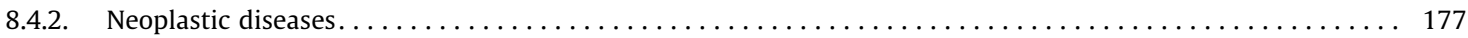

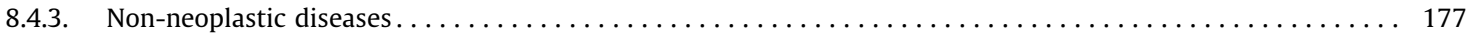

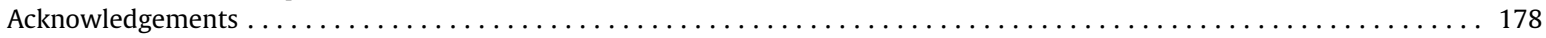

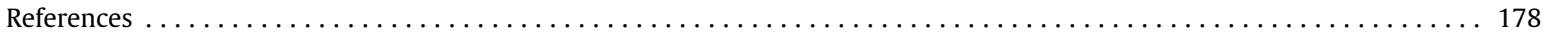

\section{Introduction}

The term metallo-proteases encompasses esopeptidase and endopeptidase involved in many biological processes, such as morphogenesis, metabolism of biologically active peptides and hormones, development, regulation of cell cycle, cell proliferation, migration, adhesion and antibiotics metabolism (Nagase, 2001). In the MEROPS database, metallo-protease families are grouped into 14 different clans, namely MA, MC, MD, ME, MJ, MK, MM, MO, MP (these requiring only one catalytic metal ion) and MF, MG, MH, MQ (these containing two metal ions acting co-catalytically). The divalent metal ion contained in the active site is in the vast majority of cases a zinc ion, but cobalt, manganese or nickel are also represented. In humans, the majority of metalloproteinases are zinc metallo-endopeptidases distributed in: clan MA (i.e., M3, M10, M12, M13, M41, M43 and M48 families), clan ME (i.e., M16 family), clan MJ (i.e., M19 and M38 families), clan MK (i.e., M22 family), clan MM (i.e., M50 family). Further, there are three families, namely M49, M76 and M79, which have not been assigned yet to a specific clan. Human metallo-peptidases play important roles in a variety of biological processes and the unbalance of their activity and expression is often at the basis of diseases like cancer, neurodegeneration, inflammation, arthritis, cardiovascular diseases. For this reason, they have been historically represented an intriguing drug targets, even though their therapeutical inhibition have raised many questions, since (i) the activity of these enzymes is pleiotropic so that their inhibition could negatively modulate some cellular functions; (ii) a large number of inhibitors are not selective for a single enzyme (Nagase, 2001). For a detailed properties of a single enzyme, it is possible to refer to the "Handbook of Proteolytic enzymes" (Barret et al., 2004).

This chapter will focus on human matrix metalloproteases, grouped as M10 family. In particular, in the first part this review briefly summarizes other families of MA clan, while in the second part attention will be concentrated on the present knowledge of members of M10 family, with a major focus on their biological functions and involvement in human diseases.

\subsection{An overview of human zinc metalloprotease grouped as clan MA}

Clan MA is the main clan of metalloproteinases and it is characterized by the "HEXXH" zinc-binding motif with two histidines acting as ligands of the catalytic $\mathrm{Zn}^{2+}$ and the glutamate as the general basis. Proto-typical proteases with this motif are: neurolysin (M3), matrix metalloproteinases (MMP, subfamily A of family M10). Here, we furnish a brief overview of this human family, discussing only representative examples of their functions.

\subsubsection{M3 family}

Human proteins included in M3 family are the soluble metallopeptidases neurolysin and thimet oligopeptidase (TOP), which reveal similar biological and biochemical features, and mitochondrial-processing-peptidase (MIP) (Table 1) (see for review Shrimpton et al., 2002; Ferro et al., 2004). Although neurolysin and TOP are well characterized biochemically, their physiopathological role has yet to be established. Both peptidases seem to play important roles in reproduction, nociception and cardiovascular homeostasis (Ferro et al., 2004). In particular, several studies indicate an involvement of neurolysin and TOP in neurotensin degradation (by exerting a broad range of endocrine and cardiovascular effect, such as hypotension, analgesia and hypothermia) and in bradykinin degradation, postulating a direct involvement in blood pressure regulation (Chabry et al., 1990; Checler et al., 1995; Davis et al., 1992; Genden and Molineaux, 1991; Kadonosono et al., 2007; Norman et al., 2003). Additionally TOP inactivates opioids and the gonadotrophin-releasing hormone, suggesting a putative role in the modulation of nociception and reproductive physiology (Cyr et al., 2010; Kest et al., 1991; Shrimpton et al., 2002). 
Table 1

Association of family grouped as clan MA with human diseases.

\begin{tabular}{|c|c|c|c|c|}
\hline Family & Subfamily & $\begin{array}{l}\text { Human } \\
\text { proteins }\end{array}$ & Pathology association & References \\
\hline M3 & & $\begin{array}{l}\text { TOP, neurolysin, } \\
\text { MIP }\end{array}$ & $\begin{array}{l}\text { Cancer }^{\mathrm{a}} ; \text { Schizophrenia } \\
\text { disease }^{\mathrm{c}} ; \text { Mithocondrial Disease }^{\mathrm{d}}\end{array}$ & $\begin{array}{l}\text { a Paschoalin et al. (2007); ' } \text { Widerlöv et al. (1982); }{ }^{\text {CPollio }} \\
\text { et al. (2008); }{ }^{\text {d}} \text { Gakh et al. (2002) }\end{array}$ \\
\hline \multirow[t]{3}{*}{ M12 } & A & Astacins & $\begin{array}{l}\text { Roles in ECM formation and morphogenesis }{ }^{\mathrm{a}} \text {; } \\
\text { Antifibrotic-target }^{\mathrm{a}} \text {; Renal failure }{ }^{\mathrm{b}} \text {; Diabetic } \\
\text { nephropahty }^{\mathrm{c}}\end{array}$ & $\begin{array}{l}{ }^{a} \text { Hopkins et al. (2007); bi et al. (2008); }{ }^{\mathrm{c}} \text { Oneda et al. } \\
\text { (2008) }\end{array}$ \\
\hline & $\mathrm{B}$ & ADAMs & 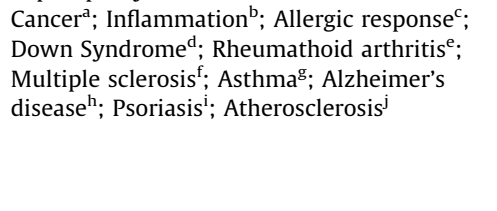 & 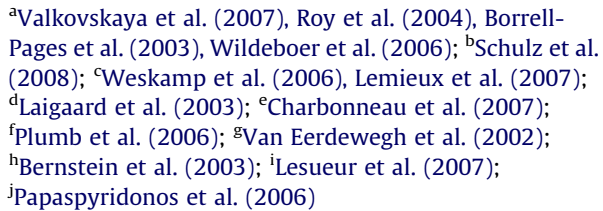 \\
\hline & & ADAMTS & $\begin{array}{l}\text { Arthritis }^{\mathrm{a}} \text {; Atherosclerosis } \\
\text { Thrombocytopenic Purpura }{ }^{\mathrm{c}} \text {; Ehler-Danlos } \\
\text { syndrome type } 7 C^{\mathrm{d}} \text {; } \text { Cancer }^{\mathrm{e}}\end{array}$ & $\begin{array}{l}\text { a'Song et al. (2007), Stanton et al. (2005), Luan et al. } \\
\text { (2008); 'Jonsson-Rylander et al. (2005), Wågsäter et al. } \\
\text { (2008), Moriguchi-Goto et al. (2009); 'Moake (2004), } \\
\text { Shenkman (2006); ' }{ }^{\text {C}} \text { Colige et al. (2004); ' }{ }^{2} \text { Dunn et al. } \\
\text { (2006), Rocks et al. (2008) }\end{array}$ \\
\hline M13 & & $\begin{array}{l}\text { Neprilysin, PEX, } \\
\text { KELL, ECE1-ECE2, } \\
\text { ECEL1 }\end{array}$ & 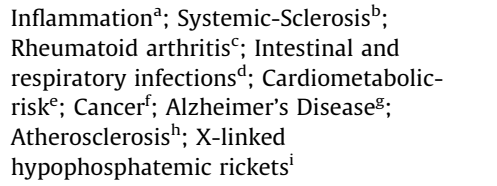 & 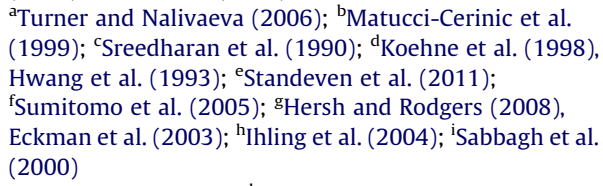 \\
\hline M41 & & Paraplegin, AFG3L2 & $\begin{array}{l}\text { Hereditary Spastic Paraplegia }{ }^{\mathrm{a}} \text {; Autosomal- } \\
\text { dominant Spinocerebellum Ataxia } 28^{\mathrm{b}}\end{array}$ & ${ }^{\mathrm{a}}$ Koppen et al. (2007); ${ }^{\mathrm{b}}$ Di Bella et al. (2010) \\
\hline M43 & & Pappalysin 1 and 2 & $\begin{array}{l}\text { Down Syndrome-marker }{ }^{\mathrm{a}} ; \text { Atherosclerosis } \\
\text { marker }^{\mathrm{b}} \text {; Pre-eclampsia }\end{array}$ & $\begin{array}{l}\text { a Van Heesch et al. (2010); }{ }^{b} \text { Consuegra-Sanchez et al. } \\
\text { (2009b); ' Carty et al. (2008) }\end{array}$ \\
\hline M48 & A & $\begin{array}{l}\text { Farnesylated- } \\
\text { protein converting } \\
\text { enzyme } 1\end{array}$ & $\begin{array}{l}\text { Hutchinson-Gilford Progeria Syndrome } \\
\text { Mandibuloacral Dysplasia }{ }^{\mathrm{b}} \text {; Restrictive } \\
\text { Dermopathy }^{\mathrm{c}}\end{array}$ & $\begin{array}{l}{ }^{a} \text { Capell et al. (2007); b Agarwal et al. (2003a,b); }{ }^{\mathrm{C}} \text { Capell } \\
\text { and Collins (2006) }\end{array}$ \\
\hline
\end{tabular}

\subsubsection{M12 family}

Human members of M12 family are classified in two subfamilies, A and B, which include astacins, ADAM and ADAMTS proteases, respectively (see for review Edwards et al., 2008; Klein and Bischoff, 2011; Mochizuki and Okada, 2007; Murphy, 2008; Tousseyn et al., 2006; Van Goor et al., 2009).

$A D A M$ (a disintegrin and metalloprotease) proteases are type 1 transmembrane proteins, defined by a modular structure encompassing (i) a prodomain, whose removal in the trans-Golgi network by pro-protein convertases is a key step in protease activation (with the exception of ADAM8 and ADAM28, which are activated by an autocatalytic cleavage), (ii) a Znbinding domain, (iii) a disintegrin domain implicated in ADAMs-integrin interaction, (iv) a cysteine-rich domain which can interact with ECM proteins like fibronectin (as in the case of ADAM13) and also binds to syndecan cell surface proteoglycans (as for ADAM12), (v) a epidermal growth factor (EGF)-like domain, (vi) a transmembrane and (vii) a cytoplasmatic tail that acts as binding site for SH3-domain containing proteins (Gaultier et al., 2002; Howard et al., 2000; Lum et al., 1998; Schlomann et al., 2002; Seals and Courtneidge, 2003; Thodeti et al., 2003; White, 2003; White et al., 2005). Only half of known ADAMs are enzymatically active, since some of them lack one or more critical catalytic residues (Van Goor et al., 2009). ADAM biological functions have been generally linked to fertility, cell adhesion and fusion, cell fate determination in nervous system, aspects of immunity (Edwards et al., 2008). Several data support the roles of at least three ADAMs in the fertilization process; in particular, ADAM2 (fertilin $\beta$ ) and ADAM3 (cyritestin) knockout mice show reduced spermatozoon binding to oolemma and also poor adherence to the zona pellucida, while ADAM1-deficient mice show reduced egg migration from uterus to the oviduct (Cho et al., 1998; Nishimura et al., 2001, 2004). ADAM1 and ADAM3 are apparently non functional in humans, since their absence does not impair fertility because it is presumably compensated by other enzymes, such ADAM21 and ADAM30 (Grzmil et al., 2001; Jury et al., 1997). One of the major ADAM functions is the ectodomain shedding of a broad spectrum of transmembrane proteins substrates, including epidermal growth factor (EGFR) ligands, tumor necrosis factor $\alpha$ (TNF- $\alpha$ ), cell adhesion molecules like CD44 and cadherins (Blobel, 2005; Nagano et al., 2004; Reiss et al., 2005). Although several ADAMs have sheddase function, the archetypal activity is shown by ADAM17 (known as TNF- $\alpha$-converting-enzyme, TACE), which is involved in immune and inflammatory response trough activation of TNF- $\alpha$ and plays a relevant role in the development via EGFR activation (Blobel, 2005). ADAM10 is the main ADAM sheddase involved in Regulated Membrane Proteolysis (RIP), the latter being defined as a pathway characterized by an ectodomain shedding on transmembrane protein followed by a cleavage within membrane itself, with critical role in Notch/Delta and Eph/ephrin signaling (Edwards et al., 2008; Saftig and Hartmann, 2005). In this context, ADAMs seem to be involved in neurogenesis, axon extension, adipogenesis and lung, heart and pancreas morphogenesis (Tousseyn et al., 2006; Van Goor et al., 2009). 
Furthermore, ADAM family has been implicated in a number of human diseases, such as cancer, asthma, infection and inflammation, neurodegeneration, thus representing attractive targets for novel therapies (Mochizuki and Okada, 2007) (Table 1). As a matter of fact, several ADAMs have been associated with cancer development and progression; for example ADAM12 is upregulated in hematologic, breast and gastric malignancies (Carl-McGrath et al., 2005; Lendeckel et al., 2005; Wu et al., 1997) and ADAM10 is highly expressed in pheochromocytoma and neuroblastoma (Yavari et al., 1998). ADAMs involvement in tumor biology can be explained by different mechanisms, since (i) ADAMs (i.e., ADAM9) could cleave ECM components, such as laminin, and promote cell invasion, similarly to MMPs; (ii) the shedding of adhesion molecules could affect cell adhesion to vasculature; (iii) the shedding of growth factors and their receptors may alter cell growth, as documented for ADAM17 and TNF- $\alpha$ in breast cancer (Kenny and Bissell, 2007; Mazzocca et al., 2005; Tousseyn et al., 2006). The shedding of adhesion molecules directly links ADAMs with inflammatory response, being involved in leukocyte recruitment (Garton et al., 2006; Hafezi-Moghadam and Ley, 1999; Schulz et al., 2008). Linkage studies have also demonstrated that ADAM33 is a candidate asthma-susceptibility locus, even though its function in asthma is unknown (Van Eerdewegh et al., 2002). Interestingly, the identification of ADAM9, ADAM10 and ADAM17 as $\alpha$-secretases, involved in non-amyloidogenic processing of $\beta$-amyloid precursor protein (APP), highlights a new therapeutical approach for Alzheimer's disease based on the modulation of ADAMs functional profile (Fahrenholz and Postina, 2006; Kojro and Fahrenholz, 2005).

ADAMTS (a disintegrin and metalloprotease with thrombospondin motif) are a group of proteins closely related to ADAMs (see for a review Apte et al., 2009; Jones and Riley, 2005; Li and Liu, 2010; Porter et al., 2005; Salter et al., 2010; Shiomi et al., 2010; Tortorella et al., 2009; Wagstaff et al., 2011).

Unlike ADAMs, ADAMTS are secreted proteins and possess: (i) a thrombospondin type-like (TS) repeat between the disintegrin-like and the cysteine-rich domain; (ii) several thrombospondin-like repeats in the C-terminal region, which seem to be important to bind ECM components (Apte, 2009; Hashimoto et al., 2004; Kuno et al., 2000). These enzymes play roles in development, angiogenesis and coagulation and their dysregulation have been implicated in many disease processes, such as inflammation, cancer, arthritis and atherosclerosis ( $\mathrm{Li}$ and $\mathrm{Lu}, 2010$ ) (Table 1). ADAMTS13 has been identified as the von Willebrand factor (VWF)-cleaving protease, whose substrate is a carrier protein for clotting factor VIII, which mediates platelet adhesion to areas of vascular damage (Fujikawa et al., 2001; Levy et al., 2001; Sadler et al., 2000). ADAMTS13 deficiency, determined by anti-ADAMTS13 autoantibody formation or gene mutations, can lead to thrombotic thrombocytopenic purpura (TPP), a condition characterized by microvascular thrombosis with renal failure, anemia and neurological disorder (Levy et al., 2001; Furlan, 1996). In addition, members of this family (such as ADAMTS1 and ADAMTS8) show a powerful angioinhibitory activity, since they suppress fibroblast growth factor-2 and vascular endothelial growth factor (VEGF)-mediated angiogenic effect on endothelial cells (Vazquez et al., 1999). Mutations in ADAMTS1 have been also associated with an increased risk of coronary artery disease and ADAMTS4, ADAMTS7 and ADAMTS8 have been implicated in atherosclerotic plaque formation (Moriguchi-Goto et al., 2009; Sabatine et al., 2008; Wågsäter et al., 2008). A subgroup of ADAMTS, also referred as "aggrecanases" (i.e., ADAMTS4 and ADAMTS5), have been involved in the degradation of the aggrecan, which forms a major component of cartilage and plays a key role in protecting collagen from degradation (Malfait et al., 2002; Pratta et al., 2003). In this context, several recent discoveries have connected ADAMTS to arthritic disease, rendering these enzymes an important target in arthritis treatment (Li and Liu, 2010).

Astacins family includes several hundred protein detected in species ranging from hydra to humans (for a review see Ge and Greenspan, 2006; Hopkins et al., 2007; Sterchi et al., 2008).

In humans, there are six astacin genes, including two meprins, three bone morphogenetic protein1 (BMP1)/Tolloid like (TLD) proteases and one ovastacin (Sterchi et al., 2008) (Table 1). BMP1 was shown to provide the procollagen C-protease activity involved in the cleavage of C-propeptide from collagen I-III (Kessler et al., 1996; Li et al., 1996). It has then become the prototype of a small subgroup of the astacin family with a similar protein domain architecture, which includes Tolloid-like 1 and Tolloid-like 2. These enzymes play a key role in ECM formation and development through the processing of a variety of precursor proteins in mature functional form (such as procollagen V, VII, XI and laminin 5) and the activation of a subset of transforming growth factor- $\beta$ (TGF- $\beta$ ) proteins. The multiple roles of BMP1/TLD-related proteins in collagen fibrillogenesis render them interesting anti-fibrotic targets and their inhibition represents a possible approach for the treatment of muscular dystrophies (Hopkins et al., 2007; Wolfman et al., 2003). Meprin proteins are distinguished from other astacins since they include a transmembrane domain and have been discovered only in vertebrates. Meprin subunits $\alpha$ and $\beta$ cleave a variety of biologically active peptides; in particular, we want to point out gastrin and cholecystokin on one side (which are substrates for meprin $\beta$ ) and substance P and many cytokines on the other side (which are substrates for meprin $\alpha$, see Sterchi et al., 2008). Meprins are expressed abundantly in epithelial cells of kidney, intestine and skin and recently a number of studies indicate their potential functions in pathological conditions, such as acute and chronic renal failures, diabetic nephropathy, colon carcinoma (Bylander et al., 2008; Lottaz et al., 1999; Red Eagle et al., 2005).

\subsubsection{M13 family}

M13 family is group of neutral endopeptidases including neprilysin (NEP), endothelin-converting enzyme (ECE1), endothelin-converting-enzyme-like 1 (ECEL1), the erythrocyte surface antigen KELL and the PHEX gene product (for a review see Kiryu-Seo and Kiyama, 2004; Lee et al., 2000; Turner, 2003; Turner and Nalivaeva, 2006). They are involved in a great number of biological processes, such as neurotransmission, reproduction, cancer progression, control of blood pressure; therefore, they are considered potential therapeutic targets in cardiovascular and inflammatory disorders (Bland et al., 2008; Turner et al., 2001) (Table 1). M13 enzymes display a short N-terminal cytoplasmic domain, a single transmembrane helix 
and a C-terminal extracellular domain containing the active site and they are generally selective inhibited by Streptomyces product phosphoramidon (Emoto and Yanagisawa, 1995; Turner and Nalivaeva, 2006).

NEP involvement in the inactivation of a variety of physiologically active peptides (such as enkephalin, substance P, bradykinin, oxytocin, neurotensin, bombesin, atrial-natriuretic-peptide (ATR) and $\beta$-amyloid) reflects its broader spectrum of physiopathological functions. It is now accepted that NEP functions in the turn off of neuropeptide signals (as for enkephalins) in a fashion similar to acetylcholinesterase at cholinergic synapses. It is located on pre- and post-synaptic membranes and on axonal membrane, where it inactivates the neuropeptide release, acting after their interaction with respective receptors (Matsas et al., 1983; Roques et al., 1980; Turner, 2003). Similarly to angiotensin-converting-enzyme (ACE), NEP inactivates ATR and thus enhances vasodilatation and natriuresis. NEP/ACE inhibitors, the so called "vasopeptidase inhibitors", are considered a novel therapeutic approach in hypertension, heart and renal disease treatment (Bralet and Schwartz, 2001; Kenny and Stephenson, 1988). Recently, a third class of inhibitors, also including the ECE inhibitor, has been developed: the dual and triple inhibitors, in addition to their ability to effectively lower blood pressure in hypertensive patients, also display antinflammatory and antifibrotic activities. However, this therapy is not free of concerns, since clinical data suggest that the incidence of angioedema may increase with vasopeptidase inhibition (Campbell, 2003; Daull et al., 2007; Quaschning, 2005). Possible NEP implication in cancer mechanism has been postulated on the basis of the NEP identity with the common acute lymphoblastic leukemia antigen CD10, a leukemia associated antigen expressed in lymphoid precursors and germinal B cells, and of an alteration of its expression and activity in a variety of malignancies (Carrel et al., 1983; Tran-Paterson et al., 1989). In particular, NEP plays a pivotal role in the development and progression of androgen-independent-prostate cancer (PC) (Osman et al., 2004; Papandreou et al., 1998; Usmani et al., 2000), since its substrates, such as bombesin and endothelin-1, are implicated at various stages of PC (Albrecht et al., 2004; Freedland et al., 2003). In recent years, NEP has also emerged as an important tumor suppressor gene product and its biological role has been not only related to its enzymatic function, but also to a direct protein-protein interaction (Sumitomo et al., 2005). For example, NEP cytoplasmic tail directly associates with the tumor suppressor PTEN, leading to a negative regulation of downstream cell growth and cell survival pathways, thereby regulating Akt/PKB signaling (Sumitomo et al., 2004). In vitro and in vivo evidences show NEP involvement in $\beta$-amyloid $(\beta A)$ catabolism and NEP is now considered one of the most important proteases targeting $\beta A$ in the extracellular space, raising the concept of its use as therapeutic target for Alzheimer's disease (Hersh and Rodgers, 2008; Iwata et al., 2000; Kanemitsu et al., 2003).

NEP homologue ECE1 has been also characterized as an A $\beta$-degrading enzyme that appears to act intracellularly, thus limiting the amount of $A \beta$ available for secretion (Eckman et al., 2001, 2003; Shirotani et al., 2001). ECE1 is also known to catalyze the final step in the biosynthesis of the potent endogenous vasoconstrictor peptide, endothelin-1, which acts in a paracrine fashion to regulate vascular tone (Matsumura et al., 1990; Xu et al., 1994). Since the upregulation of ET-1/ECE1 is present at different stages of atherosclerotic plaque evolution, it has been also suggested as a target in atherosclerosis therapy (Ihling et al., 2001, 2004). A number of other peptides are cleaved in vitro by ECE1, such as substance P, neurotensin and bradykinin, even though is not clear whether these peptides are its physiological substrates (Turner et al., 2001). Despite the structural similarities between ECEL1, ECE and NEP, ECEL1 does not cleave ECE and NEP substrates and its physiological function and substrate specificity remain unknown (Kiryu-Seo et al., 2000; Turner et al., 2001). ECEL1, specifically expressed in neurons of the central and peripheral nervous system since early developmental stages, seems to play an important role in neuronal development (Nagata et al., 2006). ECEL1 expression has been also highly associated with nerve injury in CNS and PNS, since peripheral and optic nerve transections induce ECEL1 expression in nerve-injured neurons (Kato et al., 2002; Kiryu-Seo et al., 2000).

Mutations in the PHEX gene have been identified as responsible for X-linked hypophosphatemic rickets (XLH), the most common form of inherited rickets characterized by growth retardation and rachitic and osteomalacic bone disease (Sabbagh et al., 2000; Tenenhouse, 1999). Although no endogenous substrates for PEX protein have been identified, it has been suggested that PEX could inactivate paracrine or autocrine factors involved in bone and teeth mineralization and/or circulating factors which mediate renal phosphate reabsorption and vitamin D metabolism (Lipman et al., 1998; Tenenhouse, 1999).

Kell protein is a component of the highly polymorphic Kell/XK complex, expressing over 25 antigens, that can induce severe reactions in mismatched blood-transfused patients and severe fetal anemia in sensitized mothers (Lee et al., 2000). The physiological functions of Kell and XK have not been fully elucidated, but Kell is a zinc endopeptidase with endothelin-3converting enzyme activity and XK has the structural characteristics of a membrane transporter (Redman et al., 1999; Sha et al., 2006).

\subsubsection{M41 family}

M41family includes paraplegin and its homologous AFGR3L2 (Table 1) (for a review see Rugarli and Langer, 2006; Salinas et al., 2008). Paraplegin is part of the mitochondrial AAA + protein complex which forms cylindrical hexamers on the inner mitochondrial membrane. It is implicated in the cleavage of the mitochondrial targeting sequence, in ribosome maturation, in the degradation of proteins misfolded after mitochondrial membrane transport and antioxidant defense (Esser et al., 2002; Karlberg et al., 2009; Koppen et al., 2007; Nolden et al., 2005). Mutations in paraplegin gene (SPG7) bring about around 5\% of autosomal recessive hereditary spastic paraplegia (HSP), an heterogeneous group of conditions characterized by the presence of lower limb plastic and weakness (Rugarli and Langer, 2006; Salinas et al., 2008). HSP-related mutations in SPG7-gene are associated to axonal degeneration and correlate with the onset of motor impairment (Ferreirinha et al., 2004). Recent investigations show that also AFGR3L2, which forms supercomplex with paraplegin on the inner mitochondrial membrane, 
is essential for axonal development (Maltecca et al., 2008). Interestingly, genetic and functional data demonstrate that missense mutations of AFGR3L2 lead to dominant hereditary spinocerebellar ataxia type 28 (SCA28), which is a neurological disorder characterized by cerebellar dysfunction related to Purkinje cell degeneration (Di Bella et al., 2010). This result highlights the emerging concept of mitochondrial quality control machinery in protecting human cerebellum from neurodegeneration (Di Bella et al., 2010).

\subsubsection{M43 family}

Pappalysin family includes the pregnancy-associated plasma protein-A (PAPPA) and pregnancy-associated plasma protein-E (PAPPA2) (Table 1) (for a review see Boldt and Conover, 2007; Conover, 2010; Consuegra-Sanchez et al., 2009a; Kirkegaard et al., 2010). PAPPA, originally isolated as one of the proteins synthesized in the placenta, circulating at high concentrations in pregnant women, is commonly used as biochemical marker in the screening of Down's syndrome during first-trimester of pregnancy (Lin et al., 1974; Van Heesch et al., 2010; Wald et al., 1999). PAPPA seems to be a critical determinant of growth and development through the proteolysis of insulin-growth-factor-binding proteins (IGFBPs), which bind IGFI and IGFII, thus preventing their interaction with IGF receptors (Oxvig, 2001). PAPPA-mediated IGFBP cleavage "releases" IGF for receptor activation, modulating the local availability of IGF (Byun et al., 2001; Durham et al., 1994; Laursen et al., 2000). Emerging preclinical, clinical and histopathological evidences sustain that PAPPA may serve as a marker of cardiovascular risk, reflecting the atherosclerotic plaque instability (Bayes-Genis et al., 2001; Lund et al., 2003; Consuegra-Sanchez et al., 2009a). In this context, it has been proposed that cell-associated PAPPA, enhancing local IGF actions, could amplify atherosclerosis plaque formation (Conover, 2010). Little is known on PAPPA1 homologue, PAPPA2, even though in a recent study the analysis of tissue expression patterns and biological consequences of KO gene indicate distinct physiological roles for PAPP-A2 and PAPP-A in mice (Conover et al., 2011).

\subsubsection{M48 family}

Farnesylated-protein converting enzyme 1, also known as ZMPSTE24, is a membrane protease with seven predicted membrane spans (for a review see Barrowman and Michaelis, 2009; Liu and Zhou, 2008; Young et al., 2006). It performs a critical step in the processing of prelamin $A$ in lamin $A$, a nuclear intermediate filament which provides nuclear structure and participates to heterochromatin organization and cell cycle control, removing the farnesyl tail of prelamin A (Mattout et al., 2006; Ramirez et al., 2007). ZMPSTE24 is associated directly and indirectly to human progeroid disorders (Capell and Collins, 2006; Young et al., 2005). The failure of prelamin A cleavage by ZMPSTE24 due to the absence of the cleavage site results in one form of laminopathy, Hutchinson-Gilford Progeria Syndrome (HGPS), which appear to mimick accelerated aging, being characterized by bone abnormalities, slow growth and atherosclerosis (Capell et al., 2007). Mutations in ZMPSTE24 gene also induce two secondary laminopathies, Restrictive Dermopathy (RD) and Mandibuloacral Dysplasia (MD) (Capell and Collins, 2006). RD generally results in death in uterus, since it is associated to total loss of ZMPSTE24 function, while MD is a mild condition with one partially active ZMPSTE24 allele, characterized by skeletal abnormalities, such as hypoplasia of mandible, cutaneous atrophy and progeroid feature (Agarwal et al., 2003a,b; Ahmad et al., 2010; Denecke et al., 2006; Lombardi et al., 2008; Miyoshi et al., 2008).

\section{M10 family}

M10 family includes human Zn-endopeptidases known as "Matrix metalloproteinases" (MMPs) (see Table 2), which are involved in a great variety of physiopathological processes like skeletal growth and remodeling, wound healing, cancer, arthritis and multiple sclerosis (Bafetti et al., 1998; Hirose et al., 1992; Matrisian et al., 1986; Vu et al., 1998; Wysocki et al., 1993; Nagase and Woessner, 1999; Sternlicht and Werb, 2001; Vu and Werb, 2000; Opdenakker et al., 2003; Fingleton, 2007).

Since MMPs are able to degrade basement membranes and extracellular matrix components (see Table 3 ), they have traditionally been viewed as effectors of late stage of cancer evolution. However, MMPs also have an important regulatory role, as they can modulate cytokine and chemokine activity by proteolytic processing (McQuibban et al., 2000; Opdenakker et al., 2001; Schönbeck et al., 1998; Van den Steen et al., 2000).

Up to now, at least 24 MMPs have been found to be expressed in human tissues. MMPs share high protein sequence homology and have defined domain structure; thus, according to their structural properties can be divided into eight groups, namely: (1) minimal domain-MMPs (i.e., MMP-7 and MMP-26), (2) simple hemopexin-containing domain MMPs (i.e., MMP1, MMP-8, MMP-13, MMP-18, MMP-10, MMP-12, MMP-19, MMP-20 and MMP-27), (3) gelatin-binding MMPs (i.e., MMP-2 and MMP-9), (4) furin-activated secreted MMPs (i.e., MMP-11 and MMP-28), (5) vitronectin-like insert MMP (i.e., MMP-21), (6) type I transmembrane MMPs (i.e., MMP-14, MMP-15, MMP-16 and MMP-24), (7) glycosylphosphatidylinositol-anchor linked MMPs (i.e., MMP-17 and MMP-25), (8) type II transmembrane MMPs (i.e., MMP-23A and MMP-23B) (see Fig. 1 and Table 2).

According to their substrate specificity and structural organization, MMPs are usually also classified into five main subgroups, which will be used in this work, namely:

(i) Collagenases (i.e., MMP-1, MMP-8 and MMP-13), which are able to preferentially cleave fibrillar collagen, recognizing the substrate through the haemopexin-like domain (Gioia et al., 2002; Hirose et al., 1993; Murphy et al., 1992). 
Table 2

Domain structure and classification of human MMPs.

\begin{tabular}{|c|c|c|c|}
\hline MMP group & MMP subgroup & MMP & Alternative name \\
\hline \multicolumn{4}{|c|}{ Simple hemopexin-containing MMPs } \\
\hline & \multirow[t]{3}{*}{ Collagenases } & MMP-1 & Interstitial collagenase \\
\hline & & MMP-8 & Neutrophil collagenase \\
\hline & & MMP-13 & Collagenase-3 \\
\hline & \multirow[t]{2}{*}{ Stromelysines } & MMP-3 & Stromelysin-1 (transin-1) \\
\hline & & MMP-10 & Stromelysin-2 \\
\hline & \multirow[t]{4}{*}{ Other MMPs } & MMP-12 & Macrophage metalloelastase \\
\hline & & MMP-19 & RASI-1 \\
\hline & & MMP-20 & Enamelysin \\
\hline & & MMP-27 & None \\
\hline \multicolumn{4}{|c|}{ Gelatin-binding MMPs } \\
\hline & \multirow[t]{2}{*}{ Gelatinases } & MMP-2 & Gelatinase-A \\
\hline & & MMP-9 & Gelatinase-B \\
\hline \multicolumn{4}{|c|}{ Furin-activated secreted MMPs } \\
\hline & & MMP-11 & Stromelysin-3 \\
\hline & & MMP-28 & Epilysin \\
\hline \multicolumn{4}{|c|}{ Vitronectin-like insert MMPs } \\
\hline & & MMP-21 & None \\
\hline \multicolumn{4}{|c|}{ Minimal domain MMPs } \\
\hline & \multirow[t]{2}{*}{ Matrylisins } & MMP-7 & Matrilysin-1 (Pump-1) \\
\hline & & MMP-26 & Matrilysin-2 (endometase) \\
\hline \multicolumn{4}{|c|}{ Type I-transmembrane MMPs } \\
\hline & \multirow[t]{4}{*}{ MTs-MMPs } & MT1-MMP & MMP-14 \\
\hline & & MT2-MMP & MMP-15 \\
\hline & & MT3-MMP & MMP-16 \\
\hline & & MT5-MMP & MMP-24 \\
\hline \multicolumn{4}{|c|}{ GPI-linked MMPs } \\
\hline & \multirow[t]{2}{*}{ MTs-MMPs } & MT4-MMP & MMP-17 \\
\hline & & MT6-MMP & MMP-25 (leucolysin) \\
\hline \multicolumn{4}{|c|}{ Type II-transmembrane MMPs } \\
\hline & \multirow[t]{2}{*}{ MTs-MMPs } & MMP-23A & Femalysin \\
\hline & & MMP-23B & None \\
\hline
\end{tabular}

(ii) Gelatinases (i.e., MMP-2 and MMP-9), which are able to enzymatically process various substrates of the extracellular matrix (ECM), such as collagen I and collagen IV. Beside the haemopexin-like domain, gelatinases are characterized by the presence of a "collagen binding domain" (CBD), located in their catalytic domain. CBD is made of three fibronectin II-like repeats and represents the preferential binding domain for fibrillar collagen I (Steffensen et al., 1995).

(iii) Stromelysins (i.e., MMP-3, MMP-10 and MMP-11), which are able to hydrolyse collagen IV, but do not cleave fibrillar collagen I (Nagase and Woessner, 1999).

(iv) Matrilysins (i.e., MMP-7 and MMP-26), which lack the haemopexin-like domain and are able to process collagen IV but not collagen I (Marchenko et al., 2001; Wilson and Matrisian, 1996).

(v) Membrane-type I (MT-MMPs) (i.e., MMP-14, MMP-15, MMP-16, MMP-17, MMP-24 and MMP-25), which contain at the C-terminal an additional domain, represented by an intermembrane region, completed by a short cytoplasmic tail. Only MMP-14 and MMP-16 has been shown to be able to cleave fibrillar collagen I (Ohuchi et al., 1997; Sabeh et al., 2009).

Finally, there are few MMPs, such as metalloelastase (MMP-12), RASI-1 (MMP-19), MMP-21 and MMP-28, which cannot be included in any of the above mentioned classes, and which will be treated separately.

In view of the MMP involvement in several pathological processes (see Table 4), the development of powerful and selective inhibitors might represents an important therapeutic goal, this being especially relevant for cancer therapy (Konstantinopoulos et al., 2008; Martin and Matrisian, 2007). However, recent failures in cancer clinical trials of MMP inhibitors (Coussens et al., 2002) have raised a question on MMPs druggability, mostly because of their multiple and often contradictory role in cancer disease (Overall and Kleifeld, 2006a; Fingleton, 2007).

In spite of these problematic aspects, the relevance of the physiopathological role of MMPs is unquestionable, keeping unaltered their importance as potential druggable targets, even though a better knowledge is needed to understand which (and when) a MMP is actually involved in a specific pathological process (Overall and Kleifeld, 2006a).

\subsection{Structural aspects}

Sequence comparison of MMPs reveals consistent similarities in their domain organization (Fig. 1), which is usually made by: (i) the pre-peptide involved in the pro-enzyme secretion process, (ii) the auto-inhibitory pro-domain, (iii) the 
Table 3

MMPs macromolecular substrates.

\begin{tabular}{|c|c|c|c|}
\hline \multirow[t]{2}{*}{ MMP group } & \multirow[t]{2}{*}{ MMP } & \multicolumn{2}{|l|}{ Substrates } \\
\hline & & Matrix substrates & Bioactive substrates \\
\hline \multirow[t]{13}{*}{$\begin{array}{l}\text { Simple hemopexin } \\
\text { containing MMPs }\end{array}$} & MMP-1 & $\begin{array}{l}\text { Collagens I, II, III, VII,VIII, X; gelatin; aggrecan; link } \\
\text { protein; entactin; tenascin; perlecan }\end{array}$ & $\begin{array}{l}\alpha 2-\mathrm{M} ; \alpha-\mathrm{PI} ; \alpha 1 \text {-antichymotrypsin; IGFBP- } 2 \text {, } \\
3,5 ; \text { proIL-1 } \beta ; \text { CTGF }\end{array}$ \\
\hline & MMP-8 & $\begin{array}{l}\text { Collagens I, II, III, V, VII, VIII, X; Fn; entactin; tenascin; } \\
\text { gelatin; aggrecan; link protein }\end{array}$ & $\alpha-\mathrm{PI}$ \\
\hline & MMP-13 & $\begin{array}{l}\text { Collagens I, II, III, IV, VII, IX, X, XIV; aggrecan; gelatin; Fn; } \\
\text { tenascin; osteonectin; Ln; perlecan }\end{array}$ & $\begin{array}{l}\text { CTGF; ProTGF- } \beta \text {; MCP-3; } \alpha 1- \\
\text { antichymotrypsin; plasminogen }\end{array}$ \\
\hline & MMP-3 & $\begin{array}{l}\text { Aggrecan; decorin; gelatin; Fn; Ln; collagens III, IV, V, IX, } \\
\text { X, XI; tenascin; link protein; perlecan; osteonectin; } \\
\text { entactin }\end{array}$ & $\begin{array}{l}\text { IGFBP-3; proIL- } 1 \beta ; \text { HB-EGF; CTGF; E- } \\
\text { cadherin; } \alpha 1 \text {-antichymotrypsin; } \alpha 1 \text {-PI; } \alpha 2- \\
\text { M; plasminogen; uPA; pro-MMP-1, } 7,8,9,13\end{array}$ \\
\hline & MMP-10 & $\begin{array}{l}\text { Aggrecan; Ln; Fn; gelatin; collagens III, IV, V, IX, X, XI; } \\
\text { tenascin; link protein; }\end{array}$ & Pro- $1,8,10$ \\
\hline & MMP-12 & $\begin{array}{l}\text { Elastin; aggrecan; Fn; collagen IV; gelatin; vitronectin; } \\
\text { entactin; osteonectin; Ln; nidogen }\end{array}$ & Plasminogen; apoliprotein(a) \\
\hline & MMP-19 & $\begin{array}{l}\text { Collagen IV; gelatin; Fn; tenascin; aggrecan; enatctin; } \\
\text { COMP; Ln; nidogen }\end{array}$ & $\begin{array}{l}\text { IGFBP-3; proIL-1 } \beta \text {; HB-EGF; CTGF; E- } \\
\text { cadherin; } \alpha 1 \text {-antichymotrypsin; } \alpha 1 \text {-PI; } \alpha 2- \\
\text { M; plasminogen; uPA; pro-MMP-1, 7, } 8,9,13\end{array}$ \\
\hline & MMP-20 & Amelogenin; aggrecan; gelatin; COMP & Unknown \\
\hline & MMP-27 & Unknown & Unknown \\
\hline & MMP-2 & $\begin{array}{l}\text { Gelatin; collagens I, IV, V, VII, X, XI, XIV; Ln; Fn; } \\
\text { elastin;aggrecan; osteonectin; link protein }\end{array}$ & $\begin{array}{l}\text { ProTGF- } \beta \text {; FGF receptor I; MCP-3; IGFBP-5; } \\
\text { proIL-1 } \beta \text {; galectin- } 3 \text {; plasminogen }\end{array}$ \\
\hline & MMP-9 & $\begin{array}{l}\text { Gelatin; collagens I, III, IV, V, VII, X, XII; elastin; entactin; } \\
\text { aggrecan; Fn; link protein; vitronectin; N-telopeptide of } \\
\text { collagen I }\end{array}$ & $\begin{array}{l}\text { ProTGF- } \beta \text {; IL-2 receptor a; Kit-L; IGFBP-3; } \\
\text { proIL-1 } \beta \text {; ICAM-1; } \alpha 1 \text {-PI; galectin-3; } \\
\text { plasminogen }\end{array}$ \\
\hline & MMP-11 & Fn; Ln; aggrecan; gelatins & $\alpha 1-\mathrm{PI} ; \alpha 2-\mathrm{M} ;$ IGFBP-1 \\
\hline & MMP-28 & Unknown & Casein \\
\hline $\begin{array}{l}\text { Vitronectin-like insert } \\
\text { MMPs }\end{array}$ & MMP-21 & Unknown & Unknown \\
\hline \multirow[t]{2}{*}{ Minimal domain MMPs } & MMP-7 & $\begin{array}{l}\text { Aggrecan; gelatin; Fn; Ln; elastin; entactin; collagens, III, } \\
\text { IV, V, IX, X, XI; tenascin; decorin; link protein; } \\
\text { vitronectin }\end{array}$ & $\begin{array}{l}\text { Pro } \alpha \text {-defensin; Fas-L; } \beta 4 \text { integrin; E- } \\
\text { cadherin; proTNF- } \alpha \text {; CTGF; HB-EGF; RANKL; } \\
\text { IGFBP-3; plasminogen }\end{array}$ \\
\hline & MMP-26 & Gelatin; collagen IV; Fn; fibrinogen; vitronectin & pro-MMP-9; $\alpha 1-\mathrm{PI}$ \\
\hline \multirow[t]{4}{*}{$\begin{array}{l}\text { Type I-transmembrane } \\
\text { MMPs }\end{array}$} & MT1-MMP & $\begin{array}{l}\text { Collagen I, II, III; gelatin; aggrecan; Fn; Ln; fibrin; } \\
\text { vitronectin; entactin; proteoglycans; Ln-5 }\end{array}$ & $\begin{array}{l}\text { Pro-MMP-2; Pro-MMP-13; CD44; MCP-3; } \\
\text { tissue transglutaminase }\end{array}$ \\
\hline & MT2-MMP & $\begin{array}{l}\text { Fn; tenascin; nidogen; aggrecan; entactin; collagen; } \\
\text { gelatin; perlecan; Ln; vitronectin }\end{array}$ & Pro-MMP-2; tissue transglutaminase \\
\hline & MT3-MMP & Collagen III; aggrecan; gelatin; Fn; vitronectin. & Pro-MMP-2; tissue transglutaminase \\
\hline & MT5-MMP & Gelatin;fibronectin; vitronectin; collagen, aggrecan; PG & Pro-MMP-2 \\
\hline GPI-linked MMPs & $\begin{array}{l}\text { MT4-MMP } \\
\text { MT6-MMP }\end{array}$ & $\begin{array}{l}\text { Gelatin; fibrinogen } \\
\text { Gelatin; collagen IV; fibrin; Fn; Ln }\end{array}$ & $\begin{array}{l}\text { Unknown } \\
\text { ProMMP-2 }\end{array}$ \\
\hline \multirow{2}{*}{$\begin{array}{l}\text { Type II-transmembrane } \\
\text { MMPs }\end{array}$} & MMP-23A & Unknown & Unknown \\
\hline & MMP-23B & Gelatin & Unknown \\
\hline
\end{tabular}

catalytic domain and (iv) the C-terminal haemopexin-like domain often involved in the recognition/positioning of substrates (Fig. 1).

The pro-domain, composed of about 80 residues, extends from the $\mathrm{N}$-terminus to the catalytic domain and it is responsible for the enzyme latency. A cysteine sulphydryl group present in the N-terminal pro-domain (i.e., "Cys-switch": Pro-ArgCys-Gly-X-Pro-Asp) interacts with the $\mathrm{Zn}^{2+}$ ion and blocks the active site. The pro-domain of some MMPs shows a recognition sequence for furin-like serine proteinases, which is needed for the pro-domain cleavage and, consequently, for the MMPs activation (Skiles et al., 2001).

All MMPs structures contain in the active site the common sequence motif His-Glu-X-Gly-His-X-X-Gly-X-X-His, where three His residues coordinate the catalytic $\mathrm{Zn}^{2+}$ ion (Bode et al., 1999) (Fig. 2).

The overall topology of the MMP catalytic domain is spherical, with (i) the catalytic $\mathrm{Zn}^{2+}$ ion coordinated by three His residues, (ii) a structural $\mathrm{Zn}^{2+}$ coordinated by three His and one Asp residues, and (iii) at least two structural calcium atoms octahedrally coordinated, which stabilize the whole architecture (Borkakoti, 2000).

In unliganded MMPs, the catalytic zinc residing in its center is coordinated by three imidazole $\mathrm{N}_{\varepsilon 2}$ atoms of the three His and by a fixed water molecule, which lies at a hydrogen bond distance to the carboxylate group of the catalytic Glu residue, conserved in all MMPs (see Fig. 2). Conversely, in the case of MMP complexes with bidentate inhibitors (i.e., those with an hydroxamic acid function) this water is replaced by two oxygen atoms, which together with the three imidazoles bind the 
A Minimal domain-MMPs

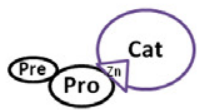

MMP7 and MMP26
Simple hemopexin domain containig MMPs

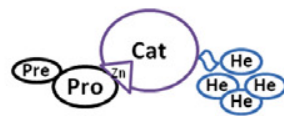

MMP1, MMP8, MMP13, MMP18,MMP10, MMP12, MMP19, MMP20 and MMP27

Gelatin- binging MMPs

Furin activated secreted MMPs

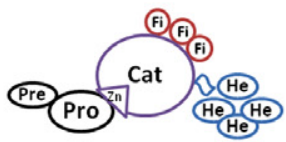

MMP2 and MMP9

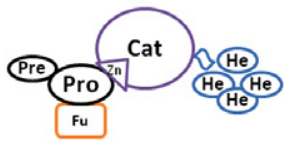

MMP11 and MMP28

Type I transmembrane MMPs

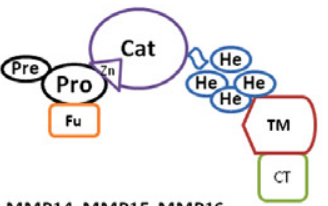

MMP14, MMP15, MMP16 and MMP24

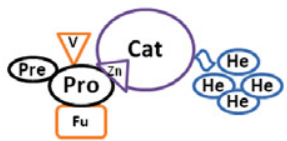

MMP21

Typell transmembrane MMPs
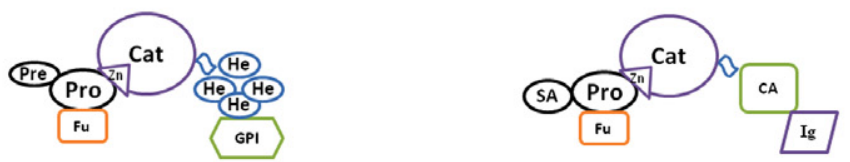

MMP17 and MMP25

MMP23A and MMP23B

legend

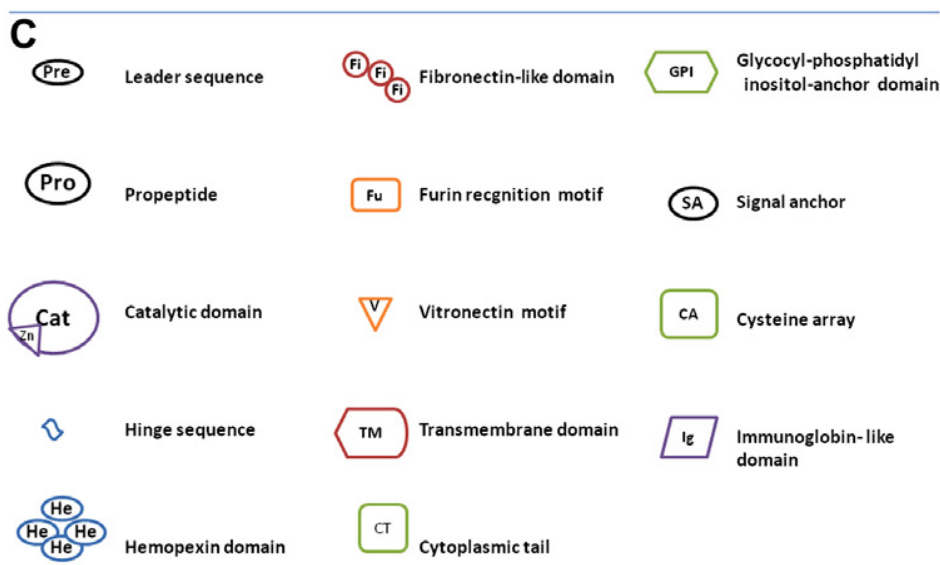

Fig. 1. Classification of human MMPs. Human MMPs are classified according to their domain composition into eight groups. Each MMP is illustrated in the latent zymogen form with the catalytic zinc $(\mathrm{Zn})$ binding to the cystein of the prodomain. 
Table 4

Involvement of different MMPs in various pathological processes.

\begin{tabular}{|c|c|c|}
\hline System & Disease & MMP \\
\hline \multicolumn{3}{|c|}{ Cardiovascular } \\
\hline & Aortic aneurysm & MMP-1 MMP-2 MMP-3 MMP-7 MMP-8 MMP-9 MMP-12 MMP-13 MMP-14 \\
\hline & Atherosclerosis & $\begin{array}{l}\text { MMP-1 MMP-2 MMP-3 MMP-8 MMP-9 MMP-10 MMP-11 MMP-12 MMP-13 } \\
\text { MMP-14 }\end{array}$ \\
\hline & Myocardial infarction & MMP-2 MMP-3 MMP-7 MMP-9 MMP-13 МMP-14 \\
\hline & Left ventricular hypertrophy & MMP-1 MMP-7 MMP-13 MMP-14 \\
\hline & Stroke & MMP-2 MMP-3 MMP-9 MMP-12 MMP-13 MMP-14 \\
\hline \multicolumn{3}{|l|}{ Nervous } \\
\hline & Alzheimer & MMP-1 MMP-2 MMP-9 MMP-24 \\
\hline & Blood-brain barrier (BBB) disruption & MMP-2 MMP-7 MMP-9 MMP-14 \\
\hline & Brain injury & MMP-7 MMP-13 \\
\hline & Encephalomyelitis & MMP-8 MMP-9 MMP-12 MMP-25 \\
\hline & HIV-dementia & MMP-7 \\
\hline & Intracerebral hemorrhage (ICH) & MMP-12 \\
\hline & Meningitis & MMP-8 MMP-12 \\
\hline & Multiple sclerosis & MMP-2 MMP-7 MMP-9 MMP-12 MMP-19 MMP-25 \\
\hline & Parkinson & MMP-3 \\
\hline & Spinal chord injury & MMP-12 \\
\hline & Vascular dementia & MMP-1 \\
\hline \multicolumn{3}{|c|}{ Joint and muscular } \\
\hline & Dupuytren & MMP-7 \\
\hline & Inflammatory myopathies & MMP-7 \\
\hline & Medial collateral ligament (MCL) injury & MMP-12 \\
\hline & Osteoarthritis (OA) & $\begin{array}{l}\text { MMP-1 MMP-2 MMP-3 MMP-7 MMP-8 MMP-9 MMP-13 MMP-14 MMP-16 } \\
\text { MMP-17 }\end{array}$ \\
\hline & Polymyositis & MMP-9 \\
\hline & Rheumatoid arthritis (RA) & MMP-1 MMP-2 MMP-3 MMP-8 MMP-9 MMP-12 MMP-13 MMP-14 MMP-19 \\
\hline \multicolumn{3}{|c|}{ Respiratory } \\
\hline & Acute respiratory distress syndrome (ARDS) & MMP-3 \\
\hline & Asthma & MMP-1 MMP-8 MMP-9 MMP-12 MMP-25 \\
\hline & $\begin{array}{l}\text { Emphysema/chronic obstructive pulmonary } \\
\text { (COP) }\end{array}$ & MMP-1 MMP-8 MMP-9 MMP-12 MMP-14 MMP-25 \\
\hline & Idiopatic pulmonary fibrosis (IPF) & MMP-1 MMP-7 MMP-12 \\
\hline & Lung fibrosis & MMP-1 MMP-7 MMP-8 MMP-12 \\
\hline \multicolumn{3}{|c|}{ Digestive } \\
\hline & Liver fibrosis & MMP-1 MMP-8 MMP-12 MMP-13 MMP-15 \\
\hline & Liver cirrhosis & MMP-8 MMP-9 \\
\hline & & MMP \\
\hline \multicolumn{3}{|c|}{ Infectious diseases } \\
\hline & Helicobacter pylori & MMP-1 \\
\hline & Mycobacterium tuberculosis & MMP-1 MMP-7 \\
\hline & Schistosoma mansoni & MMP-12 \\
\hline \multicolumn{3}{|c|}{ Autoimmune diseases } \\
\hline & Autoimmune inner ear & MMP-9 \\
\hline & Bullosus pemphigoid & MMP-9 \\
\hline & C-protein induced myocarditis & MMP-9 \\
\hline & Lupus erythematosus & MMP-2 MMP-9 \\
\hline & Sjögren Syndrome & MMP-9 \\
\hline \multicolumn{3}{|c|}{ Other diseases } \\
\hline & Diabetic nephropathy & MMP-24 \\
\hline & Endometriosis & MMP-1 MMP-2 MMP-3 MMP-7 MMP-13 МMP-24 \\
\hline & Periodontal inflammation & MMP-2 MMP-8 MMP-9 MMP-13 MMP-25 \\
\hline \multicolumn{3}{|c|}{ Wound healing } \\
\hline & & $\begin{array}{l}\text { MMP-1 MMP-2 MMP-3 MMP-8 MMP-9 MMP-10 MMP-11 MMP-13 MMP-14 } \\
\text { MMP-28 }\end{array}$ \\
\hline
\end{tabular}

catalytic $\mathrm{Zn}^{2+}$ ion in a trigonal-bipyramidal (pentacoordinate) manner. Additionally, a conserved Met residue, forming a "Met-turn", contributes to protect the catalytic zinc (Bode et al., 1999).

The catalytic domain is connected to the haemopexin-like domain by a segment, called linker (see Fig. 1) or hinge region (Maskos, 2005). This connecting linker might fulfill important functional roles rather than simply acting as physical spacer, since it turns out to be important for the enzyme stability and for the substrate degradation (Overall and Butler, 2007), this being especially true for the collagenase family (Tsukada and Pourmotabbed, 2002). 


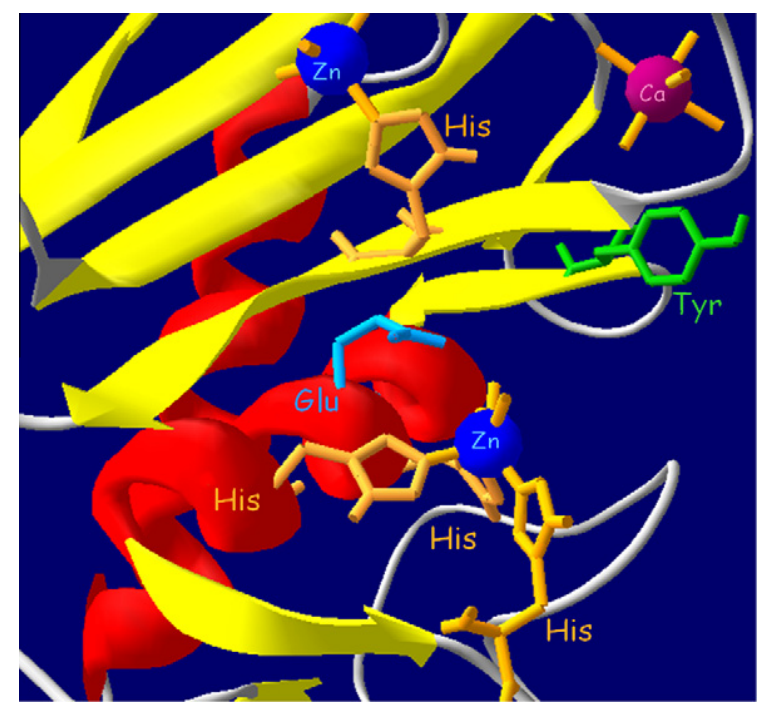

Fig. 2. Ribbon representation of the catalytic site of a MMP (in particular of MMP-8). In orange are the three histidines (His197, His201, His207 in MMP-8), coordinating the catalytic $\mathrm{Zn}^{2+}$ atom (blue sphere), and in blue the glutamate residue (Glu198 in MMP-8) involved in the proton shuttle catalytic mechanism. The magenta sphere is a structural $\mathrm{Ca}^{2+}$ atom, in green is a tyrosyl residue (Tyr189 in MMP-8) and in orange is a histidyl residue (His162 in MMP-8), which coordinates a structural $\mathrm{Zn}^{2+}$ atom (blue sphere). (For interpretation of the references to color in this figure legend, the reader is referred to the web version of this article.)

The active site cleft consists of several recognition pockets, half of which are located on the right side of the catalytic $\mathrm{Zn}^{2+}$ ion (called primed side, with pockets named $S n^{\prime}$ and $n=1,2, \ldots n$ ) and the rest on the left side of the catalytic $\mathrm{Zn}^{2+}$ ion (called unprimed side, with pockets named $S n$ and $n=1,2, \ldots n$ ). By analogy $\mathrm{P}^{\prime}$, referring to substrate and/or inhibitor, shows a generic chemical group able to fit into the $S 1^{\prime}$ pocket.

Usually, the most important recognition site (at least for small synthetic substrates and inhibitors) is the primary hydrophobic $S_{1}^{\prime}$ pocket, which is characterized by a variable length amino acid composition and conformation (according to the different MMPs), followed by shallow S2' and $\mathrm{S} 3^{\prime}$ pockets, which are primarily exposed to the solvent. For all MMPs, the size and shape of the $\mathrm{S} 1^{\prime}$ specific pocket critically regulates the ligand binding selectivity, such that MMPs generally fall in two classes (Borkakoti, 2000; Skiles et al., 2001, 2004):

1. those with a large and open S1' pocket, such as MMP-2, MMP-3, MMP-8, MMP-9, MMP-12 and MMP-13;

2. those with a small S1' pocket, such as MMP-1 and MMP-7.

In MMPs belonging to the first class, the $\mathrm{S} 1^{\prime}$ pocket is preformed, wide and more able to accommodate large P1' groups. Conversely, in MMPs belonging to the second class the $S 1^{\prime}$ pocket is smaller and not preformed, since it is occluded by either $\operatorname{Arg}^{214}$ or Tyr ${ }^{214}$, respectively (Skiles et al., 2001). Although large P1' groups are not excluded to be recognized by the socalled small S1' pocket, their binding requires an induced-fit open conformation, as observed in MMP-1 (see below).

The haemopexin-like $C$-terminal domain is composed of four $\beta$-sheets, each forming a blade of the four-bladed $\beta$-propeller structure (Bode et al., 1999). On the other hand, MMP-7 and MMP-26 are lacking the haemopexin domain, while MMP-23 has an immunoglobulin-like domain replacing the haemopexin repeat (Skiles et al., 2001).

Further, MMP-2 and MMP-9 show an additional domain, called fibronectin-like domain (see Fig. 1), which is inserted within the catalytic region and involved in collagen recognition (Skiles et al., 2001).

Moreover, some MT-MMPs contain either a membrane anchor (such as MMP-17 and MMP-25, which are anchored to the membrane through a C-terminal glycosylphosphatidylinositol moiety, and MMP-23, which is anchored to the membrane at the N-terminus) or a cytoplasmic domain at the C-terminus (such as MMP-14, MMP-15, MMP-16 and MMP-24) (Skiles et al., 2001).

The growing availability of MMP 3D structures has contributed to develop and speed up the drug design process, which can now exploit realistic molecular modeling procedures (Aureli et al., 2008; Pirard, 2007).

\subsubsection{Natural MMPs inhibitors}

Primary function of MMPs is tissue growth and remodeling by selective proteolytic degradation. Therefore, in order to avoid uncontrolled ECM turnover, inflammation, cell growth and migration, under physiological conditions, the activity of matrix metalloproteinases is accurately regulated at the levels of transcription, zymogen activation and inhibition by endogenous inhibitors.

Tissue inhibitors of metalloproteinases (TIMPs) are natural MMPs endogenous inhibitors involved in MMP-mediated ECM turnover, tissue remodeling and cellular behavior (Bourboulia and Stetler-Stevenson, 2010; Brew and Nagase, 2010). 
In humans four different TIMPs have been found, namely TIMP-1, TIMP-2, TIMP-3 and TIMP-4, which share about 40\% homology in sequencer (see Table 5). All mammalian TIMPs show two distinct domains, a C-terminal domain with about 65 residues and a N-terminal domain of about 125 amino acid residues. Each of their $\mathrm{N}$ - and C-terminal domains contains six conserved cysteine residues that form three disulfide loops. The N-terminal region binds the MMP catalytic domain and inhibits MMP activity; in particular, the TIMP inhibition mechanism is due to a bidentate coordination of the metal ion in MMPs by the N-terminal $\alpha$-amino group, thus impairing the hydrolase activity. Even though TIMP-3 is unique among human (and mammalian) TIMPs in inhibiting also several members of the ADAM and ADAMTS families and all TIMPs show a relatively low affinity for the membrane-type MMPs (i.e., MMP-14, MMP-16, MMP-24 as well as MMP-19), most of TIMPs are, under physiological conditions, broad-spectrum inhibitors, although some differences in specificity can be observed.

Furthermore, the TIMP C-terminal region interacts with pro-MMP-2 and pro-MMP-9 C-terminal hemopexin domain to stabilize the pro-enzyme. The interaction of TIMP-2 with pro-MMP-2 is part of the pro-MMP-2 activation mechanism which is observed on cell membrane surface where both membrane-type-1 MMP (MT1-MMP or MMP-14) and TIMPs play a key role in MMP-2 autolytic cleavage and activation (Itoh et al., 2001).

It should be underlined that, although TIMPs were originally characterized as MMP inhibitors, their activities only partially arise from metalloproteinase inhibition: in fact, they have various biological activities, including the modulation of cell proliferation, cell migration and invasion, anti-angiogenesis, anti- and pro-apoptosis and synaptic plasticity (Stetler-Stevenson, 2008).

\subsection{General mechanistic aspects}

MMPs catalyze the cleavage of a peptide bond by a mechanism which is similar to that reported for serine and cysteine proteinases, although different groups accomplish the electrophilic attack. In MMPs, the catalytic $\mathrm{Zn}^{2+}$ ion is coordinated by the three His residues; in the absence of a substrate or an inhibitor, a water molecule (i.e., the catalytic water) is coordinated by the $\mathrm{Zn}^{2+}$ ion as a fourth ligand, being entrapped between the metal and the catalytic Glu residue belonging to the His-GluXxx-Xxx-His-Xxx-Xxx-Gly-Xxx-Xxx-His motif (see Fig. 2). In all MMPs, the carbonyl group of the scissile peptide bond points towards the catalytic $\mathrm{Zn}^{2+}$ ion and therefore it can be polarized (Fig. 2). The peptide hydrolysis is assisted also by the relatively high $\mathrm{p} K_{\mathrm{a}}$ of the carboxyl group of the catalytic Glu residue (see Fig. 2), which becomes protonated at neutral $\mathrm{pH}$, increasing the hydroxyl character of the catalytic water molecule (Stocker and Bode, 1995). This feature facilitates the nucleophilic attack of the water molecule on the carbonyl carbon atom of the scissile peptide bond, which has a carbocationic character due to the polarization induced by the $\mathrm{Zn}^{2+}$ ion. This reaction gives rise to the tetrahedral intermediate, stabilized by both the $\mathrm{Zn}^{2+}$ ion and a carbonyl group of a nearby peptide bond, leading to the cleavage of the scissile peptide bond when a second residue (usually a residue of His, which decreases its $\mathrm{p} K_{\mathrm{a}}$ ) donates a hydrogen atom to the nitrogen atom of the imido portion of the peptide bond (Harrison et al., 1992). This sequence of events is confirmed by the dramatic reduction of the MMP activity following the Glu residue mutation (Cha and Auld, 1997). A consequence of this protonation/deprotonation sequence of events is a complex $\mathrm{pH}$-dependence of the enzymatic mechanism, which has been investigated in several MMPs (Fasciglione et al., 2000; Stack and Gray, 1990); in particular, site-directed mutagenesis has allowed to identify in MMP-3 the crucial role of $\mathrm{His}^{224}$ as a proton donor (Holman et al., 1999; Johnson et al., 2000).

A large fraction of functional studies on the enzymatic activity by MMPs has been carried out employing synthetic fluorogenic peptidic substrates (Stack and Gray, 1989; Weingarten et al., 1985). These peptides were usually based on the amino acid sequences of natural substrates, such as collagens, aiming to identify the substrate specificity pocket (see Nagase and Fields (1996) for a review). Although a comprehensive overview of all these studies is off the scope for this review, it is important to underline that this approach turned out to be very useful for the identification of the substrate recognition and the cleavage process in the immediate environment of the active site. In this respect, as an example of the relevant informations obtained, we can mention that (i) in the case of the two collagenases MMP- 1 and MMP- 8 the residue in position $\mathrm{P}^{\prime}{ }_{1}$ has been shown to play a crucial discriminatory effect, since an aromatic residue in this position renders the synthetic peptide a much better substrate for MMP-8 with respect to MMP-1 (Fields et al., 1987); (ii) in the case of the two gelatinases MMP-2 and MMP-9 a substrate selectivity (in favor of MMP-2, see Chen et al., 2002) could be only achieved by the simultaneous presence of a rather hydrophobic residue in the $\mathrm{P}_{1}^{\prime}{ }_{1}$ position and a positively charged residue (e.g., Arg) in the $\mathrm{P}_{2}$ position.

Further, investigations on the cleavage of structured synthetic substrates, derived from short triple-helical collagen-like peptides (Ottl et al., 2000), have clearly shown that the thermal stability of triple-helical synthetic substrates is a crucial aspect of the catalytic and recognition mechanism of MMP (Minond et al., 2006).

Table 5

Properties of human TIMPS.

\begin{tabular}{llll}
\hline Property & TIMP1 & TIMP2 & TIMP3 \\
\hline No. of residues & 184 & 194 & 188 \\
Approximated $M_{\mathrm{r}}$ & 21 & 22 & 22 \\
MMP inhibition & Weak for MMP-14, -16, -19, -24 & All & All \\
Pro-MMP interaction & Pro-MMP-9 & Pro-MMP-2 & Pro-MMP-2 and Pro-MMP-9 \\
\hline
\end{tabular}


Although these investigations indeed have been of the utmost importance for (i) the definition of the mechanistic steps of the proteolytic action by MMPs, (ii) the characterization of protonating groups $\mathrm{p} K_{\mathrm{a}} \mathrm{s}$ involved in the peptide bond cleavage (Fasciglione et al., 2000; Stack and Gray, 1990) and (iii) the identification of substrate recognition pockets in the close proximity of the active site, this information is not enough to account for the MMPs enzymatic activity toward macromolecular substrates. As an example of this, proteolytic cleavage of triple-helical collagen I-like peptides by collagenases is much more efficient than for stromelysins (e.g., MMP-3, see Nagase and Fields, 1996), but this difference did not explain the inability of MMP-3 to process macromolecular collagen I. Thus, functional observations on natural macromolecular substrates, such as collagens, clearly indicated that additional domains (though not directly involved in the enzymatic cleavage chemistry) are very important for substrate recognition and the enzymatic activity on macromolecules (Chung et al., 2000; Gioia et al., 2002; Murphy et al., 1992).

\subsection{Macromolecular substrates}

In the last years, evidence has been emerging that when MMPs interact with macromolecular substrates the correct active-site positioning is achieved through the interaction of exosites of MMPs with the substrates (Chung et al., 2000; Gioia et al., 2002; Murphy et al., 1992). Exosites are binding sites topologically distinct from the catalytic site (where the substrate cleavage process takes place), they can be located also in additional domains of the MMPs, playing a crucial role in the dynamic unfolding of inaccessible regions of substrates (otherwise uncleavable).

The enzymatic activity of several MMPs on different natural substrates, such as collagen I, II and IV has been investigated by several groups (Gioia et al., 2002, 2007; Patterson et al., 2001; Tam et al., 2002, 2004; Xu et al., 2004a), unraveling various functional features, which imply an important role of different domains in the macromolecular substrate proteolytic processing. Notably, MMP cleavage of bioactive substrates is temperature-dependent (Fasciglione et al., 2000; Gioia et al., 2002; Salsas-Escat et al., 2010), as also supported by the experiments on triple-helical synthetic substrates (Minond et al., 2006). Therefore, in order to derive useful informations for "in vivo" studies we think that $37^{\circ} \mathrm{C}$ should be the best experimental temperature to employ.

Collagen I is the major component of the extracellular matrix (ECM), consisting in its monomeric form of a triple helix formed by two $\alpha-1$ chains and one $\alpha-2$ chain (Prockop and Kivirikko, 1995). Collagenolytic MMPs bind native collagen I via their exosites and these interactions allow to accommodate the individual collagen $\alpha$ chain into the active site cleft of the enzyme for the cleavage process, since the intact triple helix cannot enter the active site. In the case of collagenases (i.e., MMP-1, MMP-8 and MMP-13) and of MT-MMPs (such as MMP-14) the haemopexin-like C-terminal domain is important for collagen I binding (Gioia et al., 2007; Tam et al., 2004), such that its removal (leaving only the catalytic domain) brings about an altered collagen I cleavage process (Gioia et al., 2002; Murphy et al., 1992). However, as pointed out recently, this occurrence seems to be evident only at $37^{\circ} \mathrm{C}$ (likely due to the kinetic activation process) (Salsas-Escat et al., 2010). Further, the binding affinity can be either closely similar for the two chains (as in MMP-8, see Gioia et al., 2007) or somewhat higher for the $\alpha-2$ chain (as in MMP-1 and MMP-13, see Chung et al., 2004; Coletta et al., unpublished results). On the other hand, the haemopexin-like domain does not seem to play a relevant role in substrate recognition by gelatinases, since its removal does not alter appreciably the collagen I binding (Patterson et al., 2001), whereas substrate recognition for these MMPs occurs preferentially through the fibronectin-like domain (Tam et al., 2004). On the other hand, the haemopexin-like domain of gelatinases (and in particular of MMP-2) appears to be connected instead to the lateral mobility of the enzyme along the collagen fibril surface (Collier et al., 2001). Unlike the haemopexin-like domain of collagenases (see above), the fibronectin-like domain of gelatinases shows a much higher affinity for the $\alpha-1$ chain (Gioia et al., 2007; Tam et al., 2004), clearly indicating that the structural bases for the interaction of the two types of MMPs with collagen I are different, likely involving different regions of the substrate molecule. In addition, while the interaction of the haemopexin-like domain does not bring about gross conformational changes of the triple helix of collagen I (Marini et al., 2000; Tam et al., 2004), fibronectin-like domain binding induces a substantial unwinding of the triple helix (Gioia et al., 2007; Tam et al., 2004). At $37{ }^{\circ} \mathrm{C}$ these features result in a dramatically different enzymatic mechanism between collagenases and gelatinases (in particular MMP-2) for collagen I (Gioia et al., 2007), which leads for collagenases to a specific cleavage site (giving rise to the formation of two fragments), corresponding to about 1/4 and 3/4 of the collagen chain length, (Mallya et al., 1990), while for gelatinases a widespread multiple fragmentation of the collagen molecule takes place (Gioia et al., 2007).

It is very important to point out that the interaction of MMPs and macromolecular substrates occurs in vivo in different districts and in different environmental conditions (see Table 3). Thus, during inflammation or cancer evolution pH of tissues changes quite drastically, bringing about either acidification and/or alkalinization. This may have important consequences for the interaction and the enzymatic properties of MMPs toward macromolecular substrates, as it has been observed in the case of collagen I (Gioia et al., 2010), putting in evidence as the change of $\mathrm{pH}$ may induce increase or decrease of the activity of different MMPs classes.

An additional structural element, which comes into play only for macromolecular substrates, is represented by the linker region, which does not affect the collagen recognition process, but the enzymatic cleavage process (Tsukada and Pourmotabbed, 2002). This feature indeed suggests that after the substrate recognition by collagenases through the haemopexin-like domain the enzymatic activity requires a flexible linker region to correctly position the catalytic domain onto the substrate cleavage site. This requirement seems also confirmed by the fact that the unability of MMP-3 to enzymatically process 
collagen I can be bypassed by substituting the region at the edge between the catalytic domain and the linker region with MMP-1 amino acid sequence (Chung et al., 2000).

Also in the case of collagen IV (the major component of the basement membrane) (Kühn, 1995) the role of MMPs additional domains (beside the catalytic one) seems to be very important, since binding of substrate by gelatinases indeed occurs via the interaction of the substrate with the fibronectin-like domain (Monaco et al., 2006).

In the case of macromolecular substrates an additional aspect should be taken into account; thus, unlike a synthetic substrate, a macromolecule can be processed simultaneously by more than one type of enzyme at distinct topological sites. This point may have very important consequences, as it has been shown in the case of collagen IV when it is enzymatically attacked by MMP-2 and MMP-9, which are two of the most efficient enzymes on this macromolecular substrate. In this case, for instance, the interaction site has been shown to be different and allosterically modulated (Gioia et al., 2009), such that the interaction of MMP-2 with its fibronectin-like domain brings about a dramatic increase of the MMP-9 action on the same molecule, a feature likely related to an enzyme-linked conformational change of collagen IV.

In conclusion, the functional modulation of MMPs with macromolecular substrates (as it occurs in vivo in cells and tissues) is very complex and can lead to paradoxical results. In the rest of the review we will deal with an overview of the pathological processes in which MMPs are involved. However, for the sake of clarity it must be pointed out that the investigation on and the roles of different MMPs varies very much from enzyme to enzyme, rendering very difficult a purely systematic report of the role of different MMPs. Although we have divided the review on various chapters corresponding to the classification reported above, many crossing points among different chapters will be unavoidable.

\section{Collagenases}

The name of this class of MMPs refers to the capability of its components to enzymatically process native collagen molecules without unwinding the triple helical assembly of the substrate. Their structural arrangement in the active enzyme is made by a catalytic domain (where the catalytic $\mathrm{Zn}^{2+}$ is present and the proteolytic cleavage takes place) and a hemopexinlike domain, made by a typical four-blade propeller structure; they are connected by an intermediate hinge region, which seems to play a relevant role in modulating the interplay between the catalytic and hemopexin-like domain (Tsukada and Pourmotabbed, 2002). In collagenases usually the hemopexin-like domain plays an important role in substrate recognition and correct positioning of the MMP molecule on the macromolecular substrate (Gioia et al., 2002), even though also collagenases devoid of the hemopexin-like domain are able to cleave collagen I at the primary site (Salsas-Escat et al., 2010). Thus, collagenases can degrade fibrillar collagens in their triple-helical structural assembly, bringing about the formation of thermally unstable molecules, which unwind to form gelatin to be then degraded by other proteases. The first cleavage step of type I fibrillar collagen occurs between Gly775/lle776 residues of the $\alpha 1$ (I) chain and between Gly/Leu of the $\alpha 2$ (I) chain, both corresponding to about three quarters of the distance from the $\mathrm{NH}_{2}$-terminus; after the first cleavage by collagenases we obtain the 3/4 and 1/4 fragments of the whole collagen I molecule (Mallya et al., 1990; Salsas-Escat et al., 2010).

There are three enzymes historically belonging to this class, namely (i) MMP-1 or fibroblast collagenase, (ii) MMP-8 or neutrophil collagenase, and (iii) MMP-13 or collagenase-3 (see Table 2). They are structurally similar, displaying also similar functional enzymatic properties (Nagase and Woessner, 1999), even though some slight differences can be detected for substrate specificity (Aureli et al., 2008).

Their physiological role seems to be the remodeling of the collagenous component of the extracellular matrix (ECM), thus being also involved in the wound healing process (see Table 4). As a matter of fact, wound tissues in humans and in experimental animals overexpress multiple MMPs, including mostly the three collagenases (i.e., MMP-1, MMP-8 and MMP-13, see Gill and Parks, 2008), even though some specific role can attributed to each of them (see below). Furthermore, since collagen is the predominant ECM deposit in fibrotic organs, collagenases are believed to be the main proteases responsible for the resolution of fibrosis and restoration of the normal ECM environment.

Alterations of the structure and composition of cardiomyocyte and noncardiomyocyte compartments of the myocardium appear to play a central role in the pathogenesis of heart failure (HF) associated with a number of cardiac diseases (see Table 4). Among these alterations, changes in the quantity and quality of the extracellular matrix, including the collagen network, have been characterized that induce remodeling of the myocardium and ultimately deteriorate left ventricular (LV) function and facilitate the development of HF (Lopez et al., 2010).

A very important role is played by collagenases in the atherosclerotic process, an inflammatory process that affects the vessel wall of large and medium-sized arteries, which is the primary cause of heart disease in the Western world. MMP-8 and MMP-13 collagenases are believed to be ultimately responsible for plaque destabilization and rupture as the play physiological role in the control of collagen degradation in blood vessels; conversely, MMP-1 plays a protective role in human atherosclerosis (Galis et al., 1995). In addition, all collagenases seem involved in abdominal aortic aneurysm (AAA), which is characterized by degradation of extracellular matrix, chronic inflammatory cell infiltration and extensive neovascularization (Choke et al., 2005).

MMP-1 and MMP-13 collagenases are predominantly involved in arthritic diseases. Accumulated lines of evidence have demonstrated that all collagenases are also responsible for the degradation of type II collagen (a major fibrillar collagen in the cartilage), whereas aggrecan (a major proteoglycan in the cartilage) is mostly degraded by MMP-8 and MMP-13 (Okada, 
2009). Thus, cartilage is principally degraded by MMP- 1 and MMP-13 collagenases, whereas MMP-8 shows a protective role in arthritis.

All three MMP collagenases play a role in liver fibrosis processes. Different diseases of the respiratory system are often associated with aberrant tissue remodeling by MMP-1 and MMP-8 but not by MMP-13.

Although several MMPs play a crucial role in tumor invasion and building of metastatic formations because of their ability to degrade extracellular matrix proteins, collagenases do not seem to play especially relevant roles in tumorigenesis. A variety of human neoplastic diseases have been examined for their ability to produce collagenolytic enzymes in culture, and some types of tumors of epithelial origin demonstrated a very high level of collagenolytic activity, while neoplastic diseases of mesenchymal origin, non neoplastic tissues, and other types of epithelial tumors only seldom produced collagenases. MMP-1 plays a role in initiating tumorigenesis by degrading the stroma and releasing growth factors. MMP-8 can promote tumor growth, but it also plays an anti-metastatic role in cancer. On the other hand, since MMP-13 is able to degrade basement membrane components, indeed it promotes tumor invasion and progression. MMP-13 high levels of expression in breast cancer make it a candidate as a potential tumor marker for breast cancer diagnosis. Nevertheless, MMP-1 and MMP-8 are candidates as potential markers for atypical ductal hyperplasia, respectively (Chang et al., 2009; Poola et al., 2005).

Hereafter, the three collagenases structural-functional features and their specific roles in pathological processes will be reviewed.

\subsection{MMP-1}

MMP-1 is also known as collagenase-1, fibroblast collagenase and interstitial collagenase. As many other MMPs, MMP-1 is undetectable in normal resting tissues, although in vitro is produced by a wide variety of normal cells, such as fibroblasts, macrophages, endothelial and epithelial cells. MMP-1 is mainly expressed in vivo during physiological and pathological tissue remodeling, suggesting a broad-based role in biology (Woessner and Nagase, 2000).

MMP-1 is synthesized as a single polypeptide and is secreted as a proenzyme. It comprises a major unglycosylated form of $57 \mathrm{kDa}$, and a minor glycosylated species of $61 \mathrm{kDa}$.

In normal adult tissues the levels of MMP-1 are usually low. By contrast, its expression is elevated when the system faces a disturbance, such as wound healing, repair, or remodeling processes as occur in several pathological conditions (Brinckerhoff and Matrisian, 2002).

The expression of collagenase- 1 is transcriptionally regulated by growth factors, hormones, and cytokines, and the proteolytic activity is accurately controlled through activators and inhibitors, such as $\alpha$-macroglobulins and tissue inhibitors of metalloproteinases (TIMPs) (Woessner and Nagase, 2000).

\subsubsection{Biological function}

Beside the enzymatic processing of collagen I, reported above and common to other collagenases, other matrix molecules are substrates for MMP-1, including aggrecan, versican, perlecan, casein, nidogen, serpins, and tenascin-C (McCawley and Matrisian, 2001). Therefore, MMP-1 should play a pivotal role in the physiological remodeling of extracellular matrix. Notably, MMP-1 may regulate the function of biologically active molecules by releasing them from ECM stores; for example, through degradation of perlecan MMP-1 can release bound FGF (Whitelock et al., 1996).

In the last years it has become evident that MMP-1 is also able to cleave cell surface molecules and other non-matrix substrates. Some of them comprise antichymotrypsin, antitrypsin, insulin-growth factor binding protein (IGFBP)-3, IGFBP-5, ILI $\beta$, L-selectin, ovostatin, tumor necrosis factor- $\alpha$ and stromal cell-derived factor- 1 (McCawley and Matrisian, 2001). This wide variety of substrates assigns MMP-1 as a multifunctional molecule (see Table 3). For example, MMP-1 may participate in the regulation of insulin-like growth factor (IGF) for its ability to degrade IGFBPs which bind IGF with high affinity. Likewise, MMP-1 appears to play a pivotal role in epithelial morphogenesis which depends on specific movement of epithelial cells. Thus, keratinocyte migration on collagen-1 requires specific cleavage of the collagen molecule by collagenase (Pilcher et al., 1997).

The creation of knock-out mice that are null for specific genes has been useful in attributing functions to a number of MMP family members (see Table 6). Nevertheless, this approach has not been possible in the case of MMP-1, since the human enzyme does not have a clear-cut ortholog in the adult mouse. Interestingly, the mouse ortholog (McolA) for human MMP-1 shows only 58\% amino-acid identity and is distantly related, suggesting a rapid evolution in one or both lineages (Balbin et al., 2001).

\subsubsection{Neoplastic diseases}

MMP-1 is up-regulated in a wide variety of advanced cancers, and in nearly all instances, a significant negative correlation between its expression and survival has been found (Brinckerhoff et al., 2000). Animal studies have suggested that overexpression of MMP-1 protein has a role in initiating mammary tumorigenesis by degrading stroma and releasing growth factors and other mitogens for epithelial cells (Duffy et al., 2000; Hojilla et al., 2003).

Single-nucleotide polymorphisms (SNPs) of MMP-1 seems to increase cancer risk of digestive tract tumors (Langers et al., 2011) and recently a -1607 1G-to-2G MMP-1 polymorphism has been associated with susceptibility to both development and progression of lung cancer (Liu et al., 2011). 
Table 6

Phenotypes of MMP knock-out mice.

\begin{tabular}{|c|c|c|}
\hline MMP & Knock-out mice & Phenotype \\
\hline MMP-1 & None & \\
\hline MMP-2 & $M m p 2^{-1-}$ & $\begin{array}{l}\text { Reduced angiogenesis } \\
\text { Reduced hepatocyte apoptosis and necrosis in TNF-induced } \\
\text { hepatitis } \\
\text { Increased immune-complex-induced arthritis } \\
\text { Reduced functional recovery from spinal-cord injury } \\
\text { Delayed mammary gland differentiation } \\
\text { Increased allergen-induced asphyxiation } \\
\text { Reduced intraluminal leukocytes }\end{array}$ \\
\hline MMP-3 & $M m p 3^{-I-}$ & $\begin{array}{l}\text { Accelerated mammary gland adipogenesis } \\
\text { Resistance to contact dermatitis } \\
\text { Decreased immune-complex-induced lung injury } \\
\text { Reduced hepatocyte apoptosis and necrosis in TNF-induced } \\
\text { hepatitis } \\
\text { Impaired ex vivo herniated disc resorption } \\
\text { Reduced neutrophil counts } \\
\text { Delayed incisional wound healing }\end{array}$ \\
\hline MMP-7 & $M m p 7^{-1-}$ & $\begin{array}{l}\text { Increased MMP-8 levels } \\
\text { Decreased MMP-13 levels } \\
\text { Defective innate intestinal immunity } \\
\text { Impaired tracheal wound repair } \\
\text { Impaired migration of neutrophils } \\
\text { Defective prostate involution } \\
\text { Impaired ex vivo herniated disc resorption } \\
\text { Lack of activated antimicrobial peptides } \\
\text { Decreased reorganization of cell functions }\end{array}$ \\
\hline MMP-8 & $M m p 8^{-1-}$ & $\begin{array}{l}\text { Defective inflammatory response } \\
\text { ApoE }{ }^{-l-} \text { developed smaller lesions with increased collagen } \\
\text { deposition in atherosclerotic plaques } \\
\text { More resistant to autoimmune encephalomyelitis } \\
\text { Differenet evolution of wound healing process } \\
\text { Protection against TNF-induced lethal hepatitis }\end{array}$ \\
\hline MMP-9 & $M m p 9^{-1-}$ & $\begin{array}{l}\text { Defect in endochondral bone formation } \\
\text { Resistance to bullosus pemphigoid } \\
\text { Resistance to aortic aneurysms } \\
\text { Prolonged contact dermatitis } \\
\text { Abnormal embryonic implantation } \\
\text { Delayed apoptosis of hypertrophic chondrocytes at the skeletal } \\
\text { growth plates } \\
\text { Deficient vascularization } \\
\text { Diminished neutrophil infiltrate in glomerular nephritis } \\
\text { Reduced respiratory failure and lung enlargement induced by IL- } \\
\text { 13, increased BAL leukocyte numbers } \\
\text { Protection from cardiac rupture after infarction } \\
\text { Lack of alveolar brochiolization in fibrosis } \\
\text { Increased BAL-cell recruitment after allergic challenge } \\
\text { Decreased lung injury by immune complexes } \\
\text { Decreased immune-complex-induced arthritis } \\
\text { Resistance to experimental autoimmune encephalitis } \\
\text { Aggressive form of a systemic autoimmune lymphoproliferative } \\
\text { syndrome }\end{array}$ \\
\hline
\end{tabular}

\footnotetext{
MMP-10 None

MMP-11 $\quad$ Mmp11 $^{-1}$

Accelerated neointima formation after vessel injury Increased fat excess and adipogenesis

MMP-12 Mmp12-1-

Reduced elastinolytic capacity of macrophages

Reduced macrophage recruitment into aneurysmall lesions to migrate through matrix

Resistance to cigarette-smoke-induced emphysema

Reduced $\mathrm{CaCl}_{2}$-induced aortic aneurysm

Reduced atherosclerotic lesions

MMP-13 Mmp13-1-

Abnormal skeletal growth plate development

Resistance to osteoarthritic cartilage erosion

Reduced liver fibrosis induced by cholestasis
}

Reduced intestinal adenoma formation Attenuation of cardiac hypertension and hypertrophy

Increased skin carcinogenesis in mice

Reduced skin carcinogenesis

Reduced pancreatic carcinogenesis

Reduced experimental metastasis

Reduced mammary carcinogenesis Decreased tumor cell survival and growth Increased number of metastasis 
Table 6 (continued)

\begin{tabular}{|c|c|c|c|}
\hline MMP & Knock-out mice & Phenotype & Effect on tumor development \\
\hline MMP-14 & Mmp14 $14^{-1-}$ & $\begin{array}{l}\text { Severe abnormalities in bone and connective tissue formation } \\
\text { Altered remodeling of atherosclerotic lesion } \\
\text { Resistance to aortic aneurysm formation } \\
\text { Abnormal space-temporal modulation of growth plate } \\
\text { development } \\
\text { Defective angiogenesis } \\
\text { Premature death } \\
\text { Spontaneous retarded lung alveolar development and diminished } \\
\text { surface area }\end{array}$ & \\
\hline MMP-15 & None & & \\
\hline MMP-16 & None & & \\
\hline MMP-17 & None & & \\
\hline MMP-19 & Mmp19-1- & Spontaneous adipocyte hypertrophy & $\begin{array}{l}\text { Increased early angiogenetic response and } \\
\text { tumor invasiveness } \\
\text { Decreased clinical carcinogenesis }\end{array}$ \\
\hline MMP-21 & None & & \\
\hline MMP-23A & None & & \\
\hline MMP-23B & None & & \\
\hline MMP-24 & Mt5-Mmp $p^{-1-}$ & Decreased nerve-fiber sprouting and neural invasion & \\
\hline MMP-25 & None & & \\
\hline MMP-26 & None & & \\
\hline MMP-27 & None & & \\
\hline MMP-28 & None & & \\
\hline
\end{tabular}

In breast cancer expression levels of MMP-1 have been found altered, suggesting some role for this collagenase (Köhrmann et al., 2009). Finally, since MMP-1 is one of the most highly upregulated mRNAs in atypical ductal hyperplasia cancer (ADHC), MMP-1 could potentially be used as a diagnostic marker for screening atypical ductal hyperplasia (ADH) and nonADH benign tissues and identifying patients with lesions that may develop into cancer (Poola et al., 2005).

\subsubsection{Non-neoplastic diseases}

3.1.3.1. Diseases of the cardiovascular system. MMP-1 is able to degrade insulin-like growth factor-binding proteins (IGFBPs) and the effect of its inhibition was investigated in cardiac hypertrophy induced by beta-adrenergic stimulation (Miura et al., 2003). Moreover, reduction of MMP-1 activity by the MMP inhibitor SI-27 induces a regression in the myocyte hypertrophy and the suppression of IGFBP-3 degradation.

Further, using a transgenic mouse model expressing human MMP-1 in cardiomyocytes, Kim and coworkers demonstrated that a direct disruption of the ECM of the heart results in loss of collagen and a deterioration of systolic and diastolic function, mimicking the progression of human heart failure (Kim et al., 2000).

3.1.3.1.1. Atherosclerosis. MMP-1 was detected in the macrophages, smooth muscle cells and endothelial cells of the atheroma (Galis and Khatri, 2002). Transgenic overexpression of human MMP-1 in macrophages of ApoE knockout mice surprisingly resulted in less advanced atherosclerosis, suggesting that MMP-1 has a protective role in human atherosclerosis, probably through tissue repair (Lemaitre et al., 2001).

3.1.3.1.2. Aneurysm. MMP-1 over-expression within AAA wall has been observed in association with infiltrates and neovascularization (Knox et al., 1997) in luminal endothelial cells (EC), aortic medial smooth muscle cells (SMC) and SMC of mature neovessels (Reeps et al., 2009).

3.1.3.2. Diseases of the nervous system. It has been reported that MMP-1 genotypes (MMP-1 2G2G, and MMP-1 $1 \mathrm{G} 2 \mathrm{G}$ ) were significantly and independently associated with vascular dementia (VAD). MMP-1 2G2G genotype is also associated with increased risk of Alzheimer disease (AD). Interestingly, the odds of VAD and AD was further increased in persons concomitantly carrying more than one MMP gene variation, compared to individuals that only had one high-risk genotype (Flex et al., 2006).

3.1.3.3. Diseases of joints and muscular system. In arthritic disease, it has been demonstrated a correlation between the increase in MMP-1 and collagen degradation suggesting that cleavage of cartilage collagen is directly related to the activity of this enzyme (Vincenti and Brinckerhoff, 2002). Cartilage destruction correlates with the concentration of MMP-1 and can be blocked by broad-range MMP inhibitors in a dose-dependent manner (Neidhart et al., 2003). 
3.1.3.3.1. Joint diseases. (a) Osteoarthritis (OA). In human OA cartilage, MMP-1 is overexpressed by chondrocytes (Okada, 2009), likely playing a direct role in collagen degradation because of its collagenolytic activity.

(b) Rheumatoid arthritis $(R A)$. The mechanism of articular cartilage destruction by proteinases is more complex in RA than $\mathrm{OA}$, and composed of three pathways: destruction from the surface of the articular cartilage by proteinases present in the synovial fluid, destruction through direct contact of proteolytic synovium and/or pannus tissue to the articular cartilage and intrinsic destruction by proteinases derived from chondrocytes (Okada, 2009). Rheumatoid synovial tissue exhibits the overproduction of all collagenases together with TIMP-1, 2 and 3 (Okada, 2009). They are secreted into the synovial fluid in the joint cavity, and they are believed to degrade the cartilage from the surface based on the imbalance between MMP and TIMP in favor of MMP (Yoshihara et al., 2000). Rheumatoid synovium is highly proteolytic and chondrocytes in RA also express MMP-1, previous findings indicated that several closely linked single-nucleotide polymorphisms (SNPs) in the MMP-1 loci have an important role in determining its circulating levels in RA (Chen et al., in press).

3.1.3.4. Wound healing. During human skin wound healing, MMP-1 released by keratinocytes is critical for re-epithelialization by promoting cell migration through binding to integrin and type I collagen (Dumin et al., 2001).

\subsubsection{Diseases of the respiratory system.}

3.1.3.5.1. Emphysema/chronic obstructive pulmonary disease. The understanding of the pathogenesis of emphysema in patients deficient in $\alpha 1$-antitrypsin provided the initial idea that a proteinase/antiproteinase imbalance was responsible for the disease (Lomas and Mahadeva, 2002). Since neutrophil elastase is inhibited by $\alpha 1$-antitrypsin, excess elastase activity was thought to be responsible for the destruction of the lung leading to emphysema. However, the development of emphysema in MMP-1 transgenic mice expanded the original concept of a trypsin/antitrypsin unbalance to a proteinase/antiproteinase imbalance theory (D'Armiento et al., 1992). Indeed, bronchoalveolar lavage (BAL) fluid from patients with emphysema contains more MMP-1 compared to fluid from healthy volunteers, and this is associated with increased secretion of active MMP-1 (Finlay et al., 1997). Moreover, immunohistochemistry on emphysematous lung tissue has shown that MMP-1 is expressed in epithelial cells, especially type II pneumocytes. Increased MMP-1 expression in the epithelial cells of smokers may result from a prolonged activation of the ERK mitogen-activated protein kinase pathway (Mercer et al., 2004). These data suggest that inflammatory cells may not be exclusively responsible for lung tissue destruction, but that MMP- 1 expression in epithelial cells can cause emphysema (Imai et al., 2001).

3.1.3.5.2. Interstitial pulmonary fibrosis. In human lung fibrosis, studies on gene and protein expression have unexpectedly shown a strong up-regulation of MMP-1 (Selman et al., 2000; Zuo et al., 2002). A paradox emerges as excessive accumulation of collagens is associated with over-expression of the cleaving enzyme not with the absence of it, as it might have been expected. Although there is no a clear explanation for this paradox, the location of the enzyme in the fibrotic lung may partially explain it. Thus, although MMP-1 was highly expressed in fibrotic lung tissue, the localization of the enzyme was noticed mainly in reactive alveolar epithelial cells (Selman et al., 2000). Furthermore, the so called fibroblast-type collagenase was virtually absent in fibroblasts from the interstitial fibrotic areas where collagens are being deposited. The role of epithelial MMP-1 is presently unknown but it might be related with cell migration as occurs with keratinocytes (Pilcher et al., 1997).

In chronic progressive fibrotic lung diseases, there are evidences that MMP-1 may be exploited as a marker, since MMP-1 and MMP-7 over-expression in the lung microenvironment allows to distinguish idiopathic pulmonary fibrosis (IPF) from other chronic lung diseases (Rosas et al., 2008).

3.1.3.5.3. Bronchial asthma. The reasons for enhanced deposition of extracellular matrix in the airways of asthmatic patients and the subsequent consequences on lung function are uncertain (Todorova et al., 2010). MMP-1 has been shown to be upregulated in patients' sputum (Rajah et al., 1996).

3.1.3.5.4. Tuberculosis. Elkington and coworkers have shown that MMP-1 is highly upregulated in tuberculosis (Elkington et al., 2011), leading to the degradation of the major lung ECM components, such as type I collagen and elastin. MMP-1 expression in epithelial cells in culture is driven by a monocyte-dependent network. Mycobacterium tuberculosis therefore drives a matrix degrading phenotype by both a direct infection of macrophages and an intercellular network that increases MMP secretion by epithelial cells. Elkington and coworkers provide evidence that $M$. tuberculosis drives the expression of MMP-1, which in turn promotes the collagen breakdown that leads to alveolar destruction in TB. These findings identify putative therapeutic targets for the prevention of TB (Elkington et al., 2011).

3.1.3.6. Diseases of the digestive system. The opposite process to the destructive degradation observed in arthritis occurs in fibrotic disorders that are characterized by exaggerated extracellular matrix accumulation. Here, at least theoretically, fibrillar collagen deposition in the scarring tissue might be partially related to a decreased collagenolytic activity (Iredale et al., 1998; Ruiz et al., 2003). These findings open new therapeutic avenues for fibrotic disorders, including gene therapy. In this context, it has been shown that gene delivery of human MMP-1, with transient over-expression of the enzyme in the liver attenuated established experimental hepatic fibrosis (Iimuro et al., 2003). However, analysis of the expression of MMP-1 in fibrotic disorders has given contradictory results, and the possibility of inhibition should be considered carefully.

Helicobacter pylori has been shown to induce MMP-1 expression in gastric epithelial cells, contributing to tissue destruction of the gastric mucosa (Krueger et al., 2006). 


\section{2. $M M P-8$}

One of the most intriguing MMPs, MMP-8, also known as collagenase-2 or neutrophil collagenase, was long thought to be expressed solely in neutrophil precursors during late myeloid maturation. However, it has become evident that MMP-8 can be expressed in a wide range of cells (e.g., maturing neutrophils, peripheral neutrophils, macrophages, plasma cells, T cells, bronchial epithelial cells, oral epithelial cells, corneal epithelial cells, colon mucosal cells, keratinocytes, endothelial cells, fibroblasts, colon myofibroblasts, smooth muscle cells, chondrocytes, mainly in the course of different inflammatory conditions). Many studies report that MMP-8 is indeed associated with a wide range of inflammatory disorders, as well as cancer progression.

Apart from the PMN-derived full-length MMP-8 (80 kDa) and its 75-kDa activated form devoid of the prodomain, several other MMP-8 species with a much smaller molecular weight (40-60 kDa) have been identified. These forms are poorly glycosylated compared to PMN-derived MMP-8 (Bergmann et al., 1998; Devarajan et al., 1991; Hanemaaijer et al., 1997; Kiili et al., 2002). Further experiments revealed that this high degree of glycosylation of PMN-derived MMP-8 is associated with the storage of the enzyme in the intracellular granules of those neutrophils. This explains why poorly glycosylated MMP-8, which is produced by other cell types, is not retained within the cell but is promptly secreted in the extracellular compartment after synthesis (Hasty et al., 1990; Smith et al., 1994). Owen and coworkers have described a PMN membrane-bound MMP-8 form (Owen et al., 2004). Membrane-associated MMP-8 appears to be more stable and more resistant to TIMP inhibition than the soluble secreted form.

The conformation of the $\mathrm{N}$-terminal portions (consequences of pro enzyme activation) is of extreme importance to the regulation of MMP-8 proteolytic activity. In fact two different forms of the MMP-8 result from activation alternately cleavage at two cleavage sites, leaving either Met-80 or Phe-79 as the N-terminal residue (Reinemer et al., 1994). The latter form is 'superactivated', as Phe-79 as forms a salt bridge with a Asp-232 and thereby prevents the N-terminal sequence from transient or other interference with active site. The result is a 3-fold increase in activity compared with activation cleavage at Met-80 (Knäuper et al., 1993). This feature is retained also for macromolecular substrates, such as collagen I (Gioia et al., 2002).

\subsubsection{Biological substrates}

Once activated, MMP-8 can cleave a wide range of substrates (see Table 3 ). Besides being an efficient collagen degrading protease (collagen type I > type III > type II), MMP-8 also cleaves a wide range of non-collagenous substrates, such as serine protease inhibitors and several chemokines. This allows MMP-8 to influence the biological activities of many of these substrates, since cleavage can either lead to their inactivation (Van den Steen et al., 2003a) or to an increase in their biological activities (Van den Steen et al., 2003b). MMP-8 is a potent collagenolytic enzyme that is involved in the pathogenesis of several inflammatory conditions.

\subsubsection{Neoplastic diseases}

Several studies support the notion that MMP-8 promotes tumor growth and metastasis and identify MMP-8 as a marker for tumor progression. For example, MMP-8 is expressed in head and neck squamous cell carcinomas (Moilanen et al., 2002), and patient serum levels correlate positively with the tumor stage (Kuropkat et al., 2004). In ovarian cancers, its expression level was found to be correlated with tumor grade and stage, as well as with poor prognosis (Stadlmann et al., 2003; Stenman et al., 2003). These data, which indicate that MMP-8 blockade might be beneficial for cancer patients, support the use of MMP-8 inhibitors in therapy. On the other hand, recent findings indicate that inhibiting MMP-8 could be counterproductive and might promote initial tumor formation and tumor spreading. Experimental carcinogenesis experiments revealed that MMP-8 deficient male mice are more prone to developing carcinogen-induced skin tumors (Balbin et al., 2003). The same study also revealed that the fibrosarcomas derived from MMP-8 deficient animals were more aggressive than fibrosarcomas isolated from wild-type animals (Balbin et al., 2003).

Additionally, MMP-8 expression levels were negatively correlated with the metastatic potential of tumor cell lines derived from breast tumors (Agarwal et al., 2003a,b). Furthermore, MMP-8 provides a protective effect in the metastatic process, decreasing the metastatic potential of breast cancer cells when it is over-expressed (Decock et al., 2008). Similarly, MMP-8 expression in the squamous cell carcinoma of the tongue is correlated with improved survival of patients and it is proposed that this protective action is probably correlated with the role of estrogen in the growth of tongue squamous cell carcinomas (Ardi et al., 2007; Korpi et al., 2008). A direct anti-metastatic role for MMP-8 was confirmed by Montel and coworkers, who observed that overexpressing MMP-8 in breast cancer cell lines leads to a decrease in metastasis, whereas knocking-down of MMP-8 using ribozyme technology results in increased metastasis (Montel et al., 2004).

\subsubsection{Non neoplastic diseases}

3.2.3.1. Diseases of the cardiovascular system. An increasing amount of clinical data supports the notion that MMP-8 is involved in the development of atherosclerosis, being ultimately responsible for plaque destabilization and rupture (Galis et al., 1995). Thus, MMP-8 can be found in atherosclerotic lesions, specifically in macrophages, smooth muscle cells and endothelial cells, and rupture prone plaques clearly displayed increased MMP-8 expression compared to stable plaques (Herman et al., 2001). Further studies have shown that vulnerable plaques also had an increased amount of activated MMP-8 (Molloy et al., 2004), and that patients with vulnerable plaques had higher MMP-8 plasma levels than patients with stable 
plaques (Turu et al., 2006). However, studies using genetically modified mice have revealed a more complex role of MMP-8 in atherosclerosis (see Table 6). apolipoprotein-E (ApoE) deficient mice develop atherosclerotic lesions similar to those observed in humans, although they do not spontaneously rupture or erode. Deficiency of MMP-8 in ApoE-null mice resulted in smaller lesions with increased collagen deposition (Laxton et al., 2009), and absence of MMP-13 (see below) led to thinner and less aligned collagen fibers in the plaque (Deguchi et al., 2005).

Although the levels of MMP-8 in biopsies from patients with abdominal aortic aneurysms (AAA) were higher than in normal aortas, there was no significant correlation between MMP-8 concentrations and AAA size (Wilson et al., 2005). A possible direct role for MMP-8 in AAA was tested in an experimental animal model. The study revealed that although neutrophil depletion, which was accompanied by a decrease in MMP-8, could diminish the probability for AAA formation, MMP-8 deficiency by itself could not affect the process, indicating that MMP-8 might be useful as a marker rather than as a target for AAA treatment (Eliason et al., 2005).

Among matrix-unrelated effects of MMPs, MMP-8 plays a very important role in the control of inflammation through chemokine cleavage (McQuibban et al., 2000; Page-McCaw et al., 2007). Of particular interest is the MMP-8 proteolytic activity on angiotensin (Ang) I (Mallat, 2009). The control of blood pressure is achieved through the modulation of Ang I cleavage by MMP-8 (Van Lint and Libert, 2006). Rupture of atherosclerotic plaques with subsequent thrombus formation is the most significant mechanism underlying clinically important ischemic events. Specific localization of elevated mRNA, protein, and activity levels of MMP-8 within atherosclerotic lesions, particularly at the shoulder regions of the fibrous cap, implies that MMP-8 plays a role in fibrous cap weakening by degrading extracellular matrix components, such as interstitial collagens. Raised intra-plaque levels of active MMP-8 were observed in patients with plaques showing histological evidence of rupture (Cheng et al., 2009). Interestingly, angiotensin II (ATII)-mediated hypertension promotes a "stable" fibrotic phenotype by inducing severe intra-plaque hemorrhages, characterized by increased degradation of interstitial collagen I via an MMPmediated (MMP-8 and MMP-13) mechanism (Cheng et al., 2009).

3.2.3.2. Diseases of the nervous system. Both human and animal studies indicate that MMP-8 is involved in several inflammatory diseases of the central nervous system (CNS). MMP- 8 was found to be up-regulated in the spinal cord of animals with experimental autoimmune encephalomyelitis (EAE) (Nygardas and Hinkkanen, 2002). A later study found a significant correlation between the expression of MMP-8 and the severity of symptoms. This study also revealed that granulocytes, and to a lesser extent macrophages and T cells, are the originating cell type (Toft-Hansen et al., 2004). Lopez-Otin and coworkers report that MMP- $8^{-I-}$ mice are more resistant to EAE than their wild-type counterparts, showing a marked reduction in CNSinfiltration cells and demyelinating lesions (Folgueras et al., 2008).

Finally, MMP-8 was found to be up-regulated in the cortex of animals with experimental bacterial meningitis (BM) (Leib et al., 2000). In line with these data, significantly higher levels of MMP-8 were found in the cerebrospinal fluid of human patients suffering from BM (Leppert et al., 2000).

3.2.3.3. Diseases of joints and muscular system. Osteoarthritis (OA) is a chronic disease of synovial joints. Although the etiology and pathogenesis of $\mathrm{OA}$ are poorly understood, it has been well established that degradation of the extracellular matrix (ECM) in articular cartilage is a major cause of joint destruction. Cartilage degradation is mediated by matrix metalloproteinases (MMPs), principally MMP-1, MMP-3, and MMP-13 (Klatt et al., 2009). However, in human OA cartilage, MMP-8 is overexpressed by chondrocytes similarly to MMP-1 (Okada, 2009).

Several findings suggest that MMP-8 has a role in rheumatoid arthritis (RA) pathogenesis. It is expressed in serum and synovial fluid from patients with RA. Fibroblast-like synoviocyte cultures from RA patients produce MMP-8 after TNF- $\alpha$ stimulation (Tchetverikov et al., 2003; Hanemaaijer et al., 1997). In addition, MMP-8 regulates the activity of several chemokines implicated in RA (Van den Steen et al., 2003a,b; Balbin et al., 2003). In addition, MMP-8 protect against inflammatory synovitis and bone erosion in the $\mathrm{K} / \mathrm{BxN}$ serum-transfer arthritis model. Its protective role in arthritis derived from the ability of this metalloprotease to induce changes in a series of inflammatory mediators namely, IL-1, pentraxin-3, and prokineticin receptor 2 in arthritic mice joints (Garcia et al., 2010).

The mechanism of articular cartilage destruction by proteinases is more complex in RA than OA, and composed of three pathways: destruction from the surface of the articular cartilage by proteinases present in the synovial fluid, destruction through direct contact of proteolytic synovium and/or pannus tissue to the articular cartilage and intrinsic destruction by proteinases derived from chondrocytes (Okada, 2009). Rheumatoid synovial tissue exhibits the over-production of MMP-8 together with TIMPs (Okada, 2009).

3.2.3.4. Wound healing. MMP-8 is upregulated in tissue remodeling processes (Balbin et al., 1998). However, activated MMP8 is more prominent in chronic than in healing wounds (Nwomeh et al., 1999), a difference that was linked to the large number of neutrophils present in chronic wounds (Impola et al., 2005). Using MMP-8 knockout mice (see Table 6), Balbin and coworkers showed that MMP-8 indeed influences the process of wound healing, because the inflammatory reaction after a subcutaneous injection of a carcinogen evolves differently in MMP-8 deficient animals (Balbin et al., 1998). Thus, MMP8 deficient mice initially display a neutrophil influx that is more diffuse, as compared to their wild-type counterparts; further, these animals are also unable to clear out the neutrophils present, resulting in an abnormally sustained inflammatory response (Balbin et al., 2003). 
Wound tissues in humans and in experimental animals over-express all collagenolytic MMPs including MMP-8. An increased neutrophil accumulation was found, however, during wound healing in mice lacking MMP-8 (Gutierrez-Fernandez et al., 2007). This collagenase expectedly plays a role in the complex process of wound healing that includes acute inflammatory reaction, regeneration of parenchyma cells, cell migration and proliferation, angiogenesis, contraction and tissue remodeling (Gill and Parks, 2008). As indicated by the sustained presence of PMNs in MMP-8 knock-out animals during the process of wound healing, MMP- 8 can also influence the immune response in the course of a chronic inflammation. Mice lacking MMP-8 show a delayed wound healing due to a persistent inflammatory infiltrates (see Table 6). Additionally, a decrease of collagen deposition has been observed in the wounds of these animals (Gutierrez-Fernandez et al., 2007). These studies indicate that MMP-8 can influence neutrophil recruitment during an acute inflammatory response, but at the same time it can help to resolve the inflammation at a later stage, as shown by the sustained number of neutrophils in MMP- 8 deficient animals during the course of a chronic inflammation. A very recent work demonstrates that exceeding physiological levels of the proteinase MMP-8 can result in a decreased collagen content, leading to impaired wound healing (Danielsen et al., 2011).

3.2.3.5. Diseases of the respiratory system. Clinical data from asthma patients suggest that levels of activated MMP-8 in bronchoalveolar lavage fluid (BALF) are an indicator of disease severity and are correlated with airway obstruction (Prikk et al., 2002). Also, MMP-8-deficient mice developed more severe inflammation than wild type mice in an allergen-induced airway inflammation model and showed more neutrophils in the bronchoalveolar lavage fluid (Gueders et al., 2005). Using an ovalbumin-sensitized (OVA) mouse model of asthma, Gueders and coworkers studied the role of MMP-8 during allergen-induced lung inflammation. Prevalence of neutrophils and eosinophils after allergen-induced bronchial inflammation was greater in MMP-8-deficient mice than in wild-type mice (Gueders et al., 2005). Interestingly, these animals also displayed increased IL4 , increased anti-OVA-specific IgEs, and enhanced Th2 recruitment, indicating that MMP-8 absence promotes a Th2 profile.

The progression of pulmonary emphysema, which is characterized by loss of lung elasticity and enlargement of alveolar airspaces, is also associated with MMP-8 presence. Whereas MMP-8 levels are significantly increased in BALF from patients with subclinical emphysema (Betsuyaku et al., 1999), levels of MMP-8 in the sputum of patients with emphysema correlate negatively with lung function if the patients have developed chronic obstructive pulmonary disease (COPD) (Culpitt et al., 2005). Finally, MMP-8 levels in BALF also correlate with the severity of bronchiectasis, a disease characterized by a permanent dilation of the bronchi due to chronic inflammation, leading to irreparable deformation of bronchial wall components (Sepper et al., 1995). Other studies identified activated epithelial cells (as well as infiltrating neutrophils and macrophages) as the cell types responsible for the de novo MMP-8 expression in the course of this pathology (Prikk et al., 2001; Zheng et al., 2002).

However, high MMP-8 levels, originating mostly from accumulated PMNs, are also often correlated with the degree of fibrosis. In patients suffering from cystic fibrosis (CF), MMP-8 levels correlate with PMN counts (Ratjen et al., 2002) and in patients with a more advanced CF disease state there is also correlation with disease severity (Power et al., 1994). High levels of neutrophils and accompanying MMP-8 also correlate with the fibrotic state in patients suffering from hypersensitivity pneumonitis (Pardo et al., 2000). Other groups have shown that MMP-8 is down-regulated during the course of lung fibrosis (Ruiz et al., 2003), which could be attributed to increased levels of TGF- $\beta$, being associated to a decrease of MMP- 8 expression (Moilanen et al., 2002; Palosaari et al., 2000). These data indicate that a shift from highly regulated MMP-8 expression to exaggerated, mainly PMN-driven, MMP-8 release might trigger the pathogenesis of fibrosis by disturbing the delicate balance that exists in matrix turnover.

The accumulation of collagen fibers in the lung interstitium is a form of abnormal repair in some respiratory diseases. Lung fibrosis can be secondary to an injury which leads to chronic inflammation. In this context, knocked-down experiments have demonstrated that the absence of MMP-8 results in antifibrotic effects by increasing IL-10 levels (Garcia-Prieto et al., 2010).

3.2.3.6. Diseases of the digestive system. Liver fibrosis develops as a result of chronic liver wound repair following diverse insults. The ultimate outcome of liver fibrosis is the formation of hepatocyte nodules encapsulated by a fibrillar scar matrix. In the liver, hepatic stellate cells and Kupffer cells are thought to be the main source of MMPs (Arthur, 2000). Infiltrated neutrophils produce MMP-8, which can contribute to the remodeling process. In liver fibrosis, hepatic stellate cells are converted to myofibroblastic cells and produce fibrillar collagens in the space of Disse.

Van Lint and colleagues showed that MMP-8-deficient mice were protected against TNF-induced lethal hepatitis (Van Lint et al., 2005). Livers of knockout mice did not show the massive influx of neutrophils seen in wild type mice, probably due to the functional link between MMP-8 and lipopolysaccharide-induced CXC chemokine, a PMN chemokine. Their work suggests that MMP-8 is involved in lipopolysaccharide-induced CXC chemokine release and, in turn, in neutrophil recruitment during inflammation. Likewise, the pivotal role of MMP-8 in lipopolysaccharide-induced CXC chemokine, CXCL5 and CXCL8 activation was reported (Tester et al., 2007).

The role of MMP-8 in regulating an innate immune response has been confirmed by several recent studies. One study showed that indeed MMP-8 affects the acute recruitment of neutrophils in a model of TNF/GalN-induced liver inflammation, in which animals develop a neutrophil-dependent lethal hepatitis. Livers of MMP-8 deficient animals did not show the characteristic massive neutrophil influx observed in wild-type animals, which enhanced survival (Van Lint and Libert, 2006). Surprisingly, MMP-8 deficiency increased neutrophil recruitment in the lung in response to intratracheal LPS administration 
(Owen et al., 2004). This indicates that the influence of MMP-8 on neutrophil migration is complex, and might depend on the stimulus, tissue and timeframe.

Reperfusion injury of the liver is believed to share similar mechanisms as seen in the brain, heart and lungs with regard to MMPs (Viappiani et al., 2006). Stimuli induced by cytokines and reactive oxygen species due to ischemic-reperfusion injury cause a release of MMP-8 from infiltrated neutrophils. This enzyme acts as inducer of cell apoptosis and ECM remodeling.

Because collagen is the predominant ECM deposit in fibrotic organs, collagenases are believed to be the main proteases responsible for the resolution of fibrosis and restoration of a natural ECM environment. It has been shown that repair after a cholestatic liver injury indeed correlates with MMP-8 activity (Harty et al., 2005). Furthermore, Siller-Lopez and coworkers showed in a very elegant fashion that adenoviral delivery of MMP-8 improves the recovery from liver cirrhosis in rats (SillerLopez et al., 2004). The increased liver cell proliferation, seen after adenoviral MMP-8 delivery (Siller-Lopez et al., 2004), indicates that MMP-8 also contributes to tissue regeneration itself, possibly by releasing ECM associated growth factors, such as HGF (Schuppan et al., 1998).

Gene therapy with MMP-8 displays improvement in liver cirrhosis, since MMP-8 regulates the degradation of excessive matrix deposition (Siller-Lopez et al., 2004). A very recent study using a combinatorial gene therapy strategy (i.e., Adenovirus-MMP-8 plus Adenovirus-human plasminogen activator) proved the therapeutical effect of MMP-8 on liver fibrosis regression, demonstrating that MMP-8 gene insertion improves neuroanatomical and neurochemical conditions in hepatic encephalopathy (Galvez-Gastelum et al., 2011).

\section{3. $M M P-13$}

MMP-13, also known as collagenase-3, is expressed in hypertrophic chondrocytes and osteoblasts during human fetal development (Johansson et al., 1997a; Ståhle-Bäckdahl et al., 1997). Increased levels of MMP-13 have been associated not only with arthritis and tumors, but also with other pathologies, including adult periodontitis (Kiili et al., 2002), ocular surface diseases (Li de et al., 2003), chronic ulcers (Vaalamo et al., 1997), and chronic inflammation of oral mucosal epithelium (Uitto et al., 1998) (see Table 4).

\subsubsection{Biological function}

There is little or no expression of MMP-13 in normal adult tissues, but the enzyme is expressed in diseases where there is a need for tissue repair or remodeling. The very restricted distribution of MMP-13 in normal tissues, coupled with its ability to degrade type II collagen and other extracellular matrix components (see Table 3), make this enzyme an interesting pharmaceutical target in a number of diseases (see below).

MMP-13 preferentially hydrolyzes type II collagen, the major collagen type of cartilage. This enzyme is 5-10 times more efficient than MMP-1 at cleaving this collagen type II but is five times less active on types I and III collagens (Knäuper et al., 1996). In contrast, MMP-1 preferentially cleaves type III collagen and MMP-8 type I collagen. MMP-13 is also active on other matrix components such as types IV, IX, X, and XIV collagens, and gelatin, fibronectin, and aggrecan (Billinghurst et al., 1997; Fosang et al., 1996; Freije et al., 1994; Knäuper et al., 1997; Mitchell et al., 1996). It is noteworthy that the gelatinolytic activity of MMP-13 is about 44 times greater than that of MMP-1. Other works have broadened the list of molecules which are susceptible to digestion by MMP-13 to include connective tissue growth factor (CTGF) and fibrinogen.

Hashimoto and colleagues have found that MMP-13 could restore the angiogenic properties of one isoform (namely VEGF165) of the vascular endothelial growth factor (VEGF) by cleaving CTGF and releasing VEGF165 from the CTGF/VEGF165 complex (Hashimoto et al., 2002). This property may be important for VEGF-induced angiogenesis in arthritic tissues: the cleavage of the CTGF/VEGF165 complex by the MMPs produced in the diseased joint could result in synovial membrane pannus invasion of the cartilage.

Fibrinogen is a glycoprotein that participates in both the cellular and the fluid phases of blood clot formation. It has been shown that MMP-13 interacts with the clotting system by proteolytically processing fibrinogen and also by inactivating factor XII (Hiller et al., 2000).

\subsubsection{Neoplastic diseases}

MMP-13 was originally found in breast carcinoma, but it is now associated with other types of human cancer, such as chondrosarcomas (Uria et al., 1998), gastric cancer (Elnemr et al., 2003), basal cell carcinomas (Hattori et al., 2003), and squamous cell carcinomas of the head and neck, larynx, vulva, and epithelium of the skin (Airola et al., 1997; Cazorla et al., 1998; Johansson et al., 1997b, 1999). An extensive overview of MMP-13 expression in malignant tumors has been recently published (Pendas et al., 2000). Because of its ability to degrade the components of the basement membrane, MMP-13 promotes tumor invasion and progression; thus, MMP-13 expression enhances invasion of human squamous cell carcinomas (Ala-aho et al., 2002a) and of human fibrosarcoma HT-1080 cells (Ala-Aho et al., 2002b). Some factors relevant to tumor progression are also regulators of MMP-13 expression, such as endostatin, a 20-kDa collagen XVIII fragment, which inhibits in vivo tumor growth. Nyberg and colleagues suggested that this factor's inhibitory activity on human tongue carcinoma cell invasion occurs by blocking the activation and catalytic activity of some MMP, including MMP-13 (Nyberg et al., 2003). Another factor, named relaxin, appears to enhance the invasiveness of some breast cancer cell lines by up-regulating MMP-13 mRNA expression in these tissues (Binder et al., 2002). Tumor hypoxia is a factor which contributes to the progression of a more malignant 
phenotype. Koong and coworkers analyzed the changes induced by hypoxia in cell lines derived from squamous cell carcinomas and found that MMP-13 was among the induced genes (Koong et al., 2000).

Glioblastoma, the most malignant type of primary brain tumor, has been shown to contain a small population of cancer stem cells, whose highly invasive potential depends on MMP-13 enzymatic activity (Inoue et al., 2010).

Dynamic reciprocal interactions between a tumor and its microenvironment affects both the establishment and progression of metastases. These interactions are in part mediated through proteolytic sculpting of the microenvironment, particularly by the matrix metalloproteinases (MMPs), with both tumors and stroma contributing to the proteolytic milieu. The influence of breast cancer metastases on the bone microenvironment that is executed via the induction of osteoblast MMP-13 with potential to enhance metastases growth is reported (Morrison et al., 2011).

A recent paper reports that MMP-13 is over-expressed in breast cancer tissue and confirmed to be a secreted protein by Western blot analysis. The high expression level of MMP-13 was also measured by immunohistochemical staining suggesting that MMP-13 has potential to be a new tumor marker for breast cancer diagnosis (Chang et al., 2009).

\subsubsection{Non-neoplastic diseases}

3.3.3.1. Diseases of the cardiovascular system.

3.3.3.1.1. Remodeling of the myocardium. The communication and chemical gradient between the endothelium to myocyte and among different myocytes is very important in maintaining the function of the organ, such as the heart. Extracellular matrix (ECM) connects the myocyte to myocyte, and to the endothelial cells (Henderson and Tyagi, 2006). A cause-effect relationship has been established between matrix metalloproteinases (MMPs) and left ventricular (LV) myocardial remodeling through the use of pharmacologic MMP inhibitors (Chapman et al., 2003). There is a disconnection/uncoupling between cardiomyocytes and endothelial cells by MMP-mediated collagen breaks (Henderson et al., 2007a). MMP-13 expression and collagen synthesis/degradation may play a role in pressure-overload induced cardiac remodeling (Henderson et al., 2007b).

3.3.3.1.2. Atherosclerosis. An enhanced expression of MMP-13 mRNA has been detected in aortas of apolipoprotein E-deficient mice with advanced atherosclerosis (Jeng et al., 1999). Yoon and colleagues reported a similar link between atherosclerosis and MMP-13 (Yoon et al., 2002). These authors discovered two polymorphisms in the MMP-13 promoter associated with atherosclerosis in the abdominal aorta of young black males. One polymorphism was associated with the PEA-3 site, but the mechanism for the polymorphism's action remains unknown.

Interstitial fibers of collagen type I in the plaque can be degraded by MMP-13 (Cheng et al., 2009) and recent studies demonstrated that MMP-13 regulates collagen content in the atheroma artery walls. Pharmacological MMP-13 inhibition yields collagen accumulation in plaques (a feature associated in humans with resistance to rupture), even in established plaques (Quillard et al., 2011).

3.3.3.1.3. Aneurysm. In human abdominal aortic aneurysms, which are lesions with the potential to rupture (Choke et al., 2005), MMP-13 is the most expressed MMP (Tromp et al., 2004). Moreover, there are differences in sex for collagen regulation during rodent experimental abdominal aortic aneurysm formation. Some studies suggest that alterations in extracellular matrix collagen turnover may be responsible for altered abdominal aortic aneurysm formation between sexes, proving that a decrease in types I and III collagen with a concurrent increase in MMP-13 in males compared with females (Cho et al., 2010).

Angiotensin II (ATII)-mediated hypertension increases the risk for acute coronary events, which may be induced by augmented collagen degradation. Interstitial fibers of collagen type I in the plaque can be degraded by MMP-13 specifically. Indeed high MMP-8 levels have been correlated with ruptured plaques in patients. Angiotensin II (ATII)-mediated hypertension increases the risk for acute coronary events, which may be caused by augmented collagen degradation.

3.3.3.2. Diseases of the nervous system. Neurovascular perturbations occurring after stroke lead to blood-brain barrier leakage, edema, hemorrhage, leukocyte infiltration, and progressive inflammatory reactions to brain injury over hours or even days after the initial stroke. Brain endothelium, astrocytes, neurons, and inflammatory-activated cells, such as neutrophils, may release MMP-13. High levels of MMP-9 and MMP-13 are involved in infarct growth in spite of thrombolytic therapy suggesting its ultra-early role in brain injury.

The use of specific brain biomarkers might aid stroke diagnosis and that approach might permit rapid referral of stroke patients to hospitals with acute treatments. It has been recently described that MMP-13 may be a promising marker of stroke outcome, as it exerts an ultra-early role in brain injury and it is very well correlated with infarct expansion evaluated by Diffusion-Weighted Image (DWI) MRI and have the potential to drive triage for thrombolysis therapy (Montaner, 2009). Synthesized MMP inhibitors and several compounds used for stroke secondary prevention, such as anti-inflammatory drugs, might decrease MMPs and improve the acute treatment of human brain ischemia without compromising the beneficial effects of matrix plasticity during stroke recovery (Morancho et al., 2010). MMPs are involved in tissue destruction produced by the neuroinflammatory response that follows ischemic stroke. In particular, within the neuroinflammatory response, high levels of MMP-13 is involved in brain tissue damage despite thrombolytic therapy, suggesting its ultra-early role in brain injury (Rosell et al., 2005). 
3.3.3.3. Diseases of joints and muscular system.

3.3.3.3.1. Joint diseases. MMP-13 is expressed by chondrocytes and synovial cells in human OA and RA and is thought to play a critical role in cartilage destruction. The recent development of a MMP-13 knockout mouse has documented the important role played by this enzyme in cartilage formation and further studies under pathological conditions should reveal the function of this enzyme in the progress of the disease (Takaishi et al., 2008) (see Table 6). Several in vitro studies have demonstrated the importance of MMP-13 in human arthritis (Bau et al., 2002; Billinghurst et al., 1997; Mitchell et al., 1996; Reboul et al., 1996). In vivo studies with animal models have confirmed the role of MMP-13 in osteoarthritis (OA) progression. In one of these studies, Otterness and coworkers injected active human recombinant MMP-13 intra-articularly into the knee joint of hamsters (Otterness et al., 2000). Collagen cleavage was limited to a thin superficial band at the cartilage surface, probably because of the strong binding of the hemopexin-domain for collagen and gelatin. The injection of MMP-13 led to cartilage collagen damage with the release of measurable collagen fragments. In another study, Neuhold and coworkers showed that the expression of a constitutively active human MMP-13 into hyaline cartilages and joints of transgenic mice resulted in erosion of the articular cartilage with excessive cleavage of type II collagen, loss of proteoglycan, and synovial hyperplasia, which are changes similar to those observed in human OA (Neuhold et al., 2001). Immunohistochemistry and in situ hybridization methodologies have been used to localize MMP-13 in normal and OA cartilage, and variable results have been obtained. Shlopov and colleagues showed that chondrocytes from areas surrounding OA lesions expressed more MMP-13 than the cells located further from the lesion (Shlopov et al., 1997). At the protein level, Moldovan and coworkers found that the MMP-13 protein was preferentially localized in the lower intermediary and deep layers (deep zone) of human OA cartilage (Moldovan et al., 1997), while Tetlow and colleagues associated the presence of MMP-13 at the superficial cartilage zone characterized by fibrillations and degenerative matrix changes (Tetlow and Woolley, 1998, 2001). Freemont and coworkers used in situ zymography to demonstrate that the distribution and amount of collagen II degrading activity varied with the extent of cartilage damage, being greatest in the deep zone and in cartilage lesions, and that MMP-13 mRNA expression distributed in parallel with the collagenase activity (Freemont et al., 1999). The MMP-13 localization in diseased cartilage is in the deep zone where type II collagen fibers are largest and chondrocytes are most able to reconstitute extracellular matrix (Fernandes et al., 1998).

Collagen II can modulate the gene expression profile of primary human chondrocytes inducing pro-inflammatory cytokines and MMP-13; thus, one way of inducing MMP-13 is by interleukin-1 $\beta$ via inflammatory signaling cascades, such as the MAPK and NFKB cascade (Klatt et al., 2009).

Others works showed that OA chondrocytes and synovial fibroblasts have impaired the receptor-mediated removal of MMP-13, a mechanism that may lead to enhanced local degradation of cartilage (Barmina et al., 1999; Walling et al., 1998). Internalization of MMP-13 is mediated by a 170-kDa receptor both in rodent cells and in human chondrocytes (Walling et al., 2003). As mentioned above, MMP-13 is expressed in RA, an arthritic disease characterized by chronic inflammation and cartilage destruction. In this disease, MMP-13 has been detected in some synovial membranes (Konttinen et al., 1999a,b; Lindy et al., 1997; Petrow et al., 2002; Tetlow and Woolley, 1998; Wernicke et al., 1996; Westhoff et al., 1999), and its expression correlated with elevated levels of systemic markers of inflammation, including erythrocyte sedimentation rate and C-reactive protein. In these diseased synovial membranes, MMP-13 was localized at the lining and sublining layers and also at the membrane/cartilage interface (Konttinen et al., 1999b; Lindy et al., 1997; Petrow et al., 2002; Westhoff et al., 1999).

The employment of an highly selective non-competitive inhibitor of MMP-13 (Johnson et al., 2007) significantly reduced the cartilage erosion in animal models of RA (Jüngel et al., 2010). The chondroprotective effects of micro-RNA inhibition of the MMP-13 expression was proved in human cartilage explants in vitro and through the development of inflammatory arthritis in mouse model in vivo (Akhtar et al., 2010).

3.3.3.4. Wound healing. Physiological wound repair is a highly regulated, complex process, which leads to formation of new tissue after injury. Proteolytic degradation of extracellular matrix is a crucial step in the healing of incisional skin wounds. Thus, healing of skin wounds is delayed by treatment with the broad-spectrum metalloproteinase (MP) inhibitor Galardin. Several matrix metallo proteinases (MMPs), including MMP-13, are expressed in the leading-edge keratinocytes of skin wounds (Juncker-Jensen and Lund, 2011). In mouse wound healing, MMP-13 plays a key role in keratinocyte migration, angiogenesis through digestion of connective tissue growth factor and contraction by activation of latent TGF- $\beta$ (Hattori et al., 2009). However, the healing process is not perfect and healing impairments can occur. Delayed healing and formation of chronic wounds has been linked to the excessive production of proteolytic enzymes leading to reduced amounts of growth factors and successive destruction of the extracellular matrix. It has been implied that there is an alteration in the normal control mechanisms regulating the levels of these enzymes. The study presented provides data on the concentration of proteases and cytokines in wound fluid from chronic when compared with acute wounds. Levels of proteases such as MMP-13 is found to be profoundly elevated in chronic when compared with acute wound fluids (Wiegand et al., 2010).

3.3.3.5. Diseases of the digestive system. Liver fibrosis develops as a result of chronic liver wound repair following diverse insults. The ultimate outcome of liver fibrosis is the formation of hepatocyte nodules encapsulated by a fibrillar scar matrix. In the liver, hepatic stellate cells and Kupffer cells are thought to be the main source of MMPs (Arthur, 2000). Hepatocytes produce MMP-13 upon injury and loss of MMP-13 has lead to attenuation of fibrosis in a mouse model of liver disease (Uchinami et al., 2006). 
A newly designed hyaluronic acid (HA)-shielded gene delivery system for systemic administration of plasmid DNA encoding MMP-13 is a promising therapeutic antifibrotic strategy. In fact, the enhanced expression of MMP-13 reduces collagen deposition and ameliorates liver fibrosis in mice. Moreover, the serum levels of aspartate transaminase were reduced to levels approaching those in untreated normal mice (Kim et al., 2011a).

\section{Gelatinases}

Gelatinases is a class of MMPs historically defined according to their affinity for denatured collagen (i.e., gelatin). This class includes two members, namely (i) gelatinase A or MMP-2 (72 and $62 \mathrm{kDa}$ for the pro-enzyme and the active enzyme, respectively); (ii) gelatinase B or MMP-9 (92-85-82 kDa for the pro-enzyme, the intermediate form and active enzyme, respectively) (see Table 2 ).

The domain composition shares with other MMPs the presence of a pro-peptide, a highly conserved N-terminal catalytic domain and a C-terminal hemopexin-like domain; however, unlike other MMPs, there is a peculiar additional exosite, inserted in the catalytic domain, which is called Collagen Binding Domain (CBD) (Nagase and Woessner, 1999) (see Fig. 1).

Gelatinases are deeply involved both in inflammatory processes and in tumor progression, being considered for long time one of the most important antitumoral target (Overall and Lopez-Otin, 2002). However, this potentiality has been recently questioned, drastically reducing its attraction as a pharmaceutical target.

In the last decades, concomitantly to the identification of novel substrates and physiopathological processes (see Tables 3 and 4), gelatinases have been identified as involved in several non-neoplastic pathologies (Rogriduez et al., 2009).

As a matter of fact, it is widely recognized that gelatinases participate to the aetiology of a plethora of pathologies and mainly in cardiovascular and auto-immune diseases which, taken altogether, represent a leading cause of mortality and morbility in Western countries. More specifically, in the case of cardiovascular diseases, gelatinases participate both to the genesis of the atherosclerotic lesions and to the acute event (i.e. stroke or myocardial infarction), which follows the fibrous cap rupture, whereas in the case of auto-immune diseases gelatinases are involved both in the generation of remnant epitopes and in the modulation of cross-talking between immune system compartments.

In conclusion, MMP-2 and MMP-9 role is supported by several in vivo and in vitro evidences, even though they follow distinct and even opposite patterns, consistently with the notion that MMP-9 has a pro-inflammatory property, whereas MMP2 has a pro-homeostatic one (Ram et al., 2006; Van den Steen et al., 2002). As a matter of the fact, MMP-9 takes part on the onset of a wider panel of pathologies (Sanchez et al., 2005).

\section{1. $M M P-2$}

\subsubsection{Structural peculiarities}

Crystallographic analysis of pro-MMP-2 and of pro-MMP-2 in complex with TIMP-2 have been solved, furnishing deep insights on the structure and on the activation mechanism (Morgunova et al., 1999, 2002). The S1 site (notation according to Schechter and Berger, 1967) in the catalytic cleft is deep and recognizes the classic substrate motif $\mathrm{PXXXH}_{\mathrm{Hy}}$, consistently with the notion that hydrophobic residues are likely to adapt in the S1 site and proline in the S3 site (Chen et al., 2003). Three other conserved motifs have been identified: they are the L/IXXX $\mathrm{Hy}_{\mathrm{y}}$, the $\mathrm{X}_{\mathrm{Hy}} \mathrm{SXL}$ and the HXXX $\mathrm{Hy}$, which, reasonably, account for the enzyme specificity (Chen et al., 2003). On the other hand, substrate specificity of MMP-2 versus the highly related MMP-9 is mainly determined by a glutamine (Gln412) residue in the S2 site: hence, targets are mainly discriminated for residues in P2 position (Chen et al., 2003; Nagase and Fields, 1996).

The CBD is composed by three type II fibronectin-like tandem modules with closely similar conformation (Morgunova et al., 1999). The fibronectin-like modules folds separately from the rest of the molecule and display peculiar sequence motifs, including four disulfide bonded cysteines and four aromatic residues which envisage a fixed conformation for the overall domain (Trexler et al., 2003). The CBD has a substantial area of contact with the catalytic domain, mainly involving the second FnII domain. That FnII domain binds preferentially to synthetic peptides with a high proportion of aromatic residues and no acidic side chains, and, being a major structural determinant of MMP-2, represents an intriguing pharmacological target (Briknarova et al., 1999; Trexler et al., 2003). The CBD is the main domain involved in substrates recognition (Gioia et al., 2007; Monaco et al., 2006; Patterson et al., 2001); as a matter of fact, MMP-2 mutants lacking the CBD lose their ability to bind to gelatin (Murphy et al., 1994; Xu et al., 2007). Therefore, the CBD-mediated recognition of specific binding sites is essential to drive the correct positioning of the catalytic domain to the adjacent cleavage site.

Differently from other MMPs, the hemopexin-like domain contribution to substrate recognition is minimal, whereas it is essential for the partial unwinding of substrates facilitating the access to hindered cleavage sites as in the case of collagen IV (Bode, 1995; Gioia et al., 2009; Monaco et al., 2006).

The propeptide domain displays a three-helix fold structure and the cystein of the conserved cystein-switch motif is the fourth ligand of the catalytic $\mathrm{Zn}^{2+}$ ion in the inactive enzyme (Morgunova et al., 2002). The activation mechanisms of MMP-2 has been described in detail. The pro-domain displacement occurs in presence of TIMP-2 and MT1-MMP at the cell surface, even though alternative mechanisms involving the MT2-MMP, thrombin and uPAR have been proposed (Koo et al., 2010; Morrison et al., 2001; Nishida et al., 2008; Visse and Nagase, 2003). The first model proposes that the C-terminal domain of a TIMP-2 molecule bound to a cell surface MT1-MMP acts as a receptor for the hemopexin-like domain of pro-MMP-2: 
a second MT1-MMP then cleaves the pro-peptide releasing an intermediate MMP-2 which is fully activated by another soluble MMP-2.

\subsubsection{Biological aspects}

MMP-2 is constitutively expressed in almost all human tissues and mainly by fibroblasts, endothelial and epithelial cells. The enzyme is detectable at significant serum concentration in physiological condition: its activity has been, insofar, linked to homeostatic functions (Van den Steen et al., 2002). MMP-2 expression is inducible by stimuli to a lesser extent with respect to other proteases, reflecting the absence of promoter elements for specific transcriptional factors activated under inflammatory conditions (Xu et al., 2004b).

The MMP-2 activity is tightly regulated by TIMPs; in particular, TIMP-2, TIMP-3 and TIMP-4 (see Table 5) display relevant affinity for the MMP-2 and their adequate secretion is required for a balanced MMP-2/TIMP ratio. Intriguingly, MMP-2 can further undergo PKC-dependent phosphorylation: this recent observation opens important perspectives about MMP-2 interaction with substrates (Sariahmetoglu et al., 2007).

MMP-2, also known as type IV collagenase, cleaves a vast repertoire of substrates, including cytokines, growth factors, receptors or binding factors (see Table 3). Therefore, the biological activity of the enzyme is associated to a plethora of physio-pathological processes and mainly: (i) neoplastic diseases (ii) cardiovascular diseases (iii) auto-immune diseases.

\subsubsection{Neoplastic diseases}

4.1.3.1. Epidemiological aspects. The identification as leading ECM degrading protease and the up-regulation in almost all cancers had historically linked MMP-2 to tumorigenesis and in particular to invasiveness and dissemination (Björklund and Koivunen, 2005; Fingleton, 2006; Liotta et al., 1980). Several attempts have been carried out to design specific inhibitors. Unfortunately they all failed in their therapeutic purpose: the redundancies of biological activities and the daedalian network of molecular interactions that have been, at least partially, unveiled likely accounts for the unsuccessful approach (Gioia et al., 2009; McCawley and Matrisian, 2001; Sato and Takino, 2010).

Even though MMP-2 is weakly inducible, it is aberrantly up-regulated in the tumor microenvironment and serum concentration is, then, dramatically high (Colotti et al., 2007): this last aspect has been addressed for the MMP-2 screening as a relevant diagnostic and prognostic marker (Patel et al., 2011). Epidemiologic studies suggest MMP-2 quantification as prognostic marker in patients with colorectal cancer, ovarian cancer and thyroid cancer (Kwon et al., 2011; Rajoria et al., 2011). Up-regulated levels of gelatinase A are observed in patients with breast, gastric, bone and melanoma tumors (Daniele et al., 2010; Gerstein et al., 2009; Kushlinsky et al., 2010; Nikkola et al., 2005; Ranogajec et al., in press; Stankovic et al., 2010). Furthermore, MMP-2 strongly contributes to prostate carcinogenesis (Littlepage et al., 2010) and in the vast majority of cases MMP-2 up-regulation is associated to poor prognosis (Offersen et al., 2010; Zhong et al., 2008). MMP-2 and cancer further registers a validation from genetic and epigenetic factors, since polymorphisms (SNPs) in the MMP-2 gene directly correlates with the pathology (Chaudhary et al., 2010; Kang et al., 2011; Scherf et al., 2010).

4.1.3.2. Tumorigenesis. In vivo and in vitro experimental models have revealed multiple and overlapping mechanisms through which MMP-2 contributes to tumorigenesis. However, unlike MMP-9 (see Section 4.1), there is no evidence about the unequivocal association of MMP-2 to a specific phase of the tumor development. MMP-2, consistently with the broad spectrum of targets, behaves as a multifunctional enzyme able to modulate the initial as well as the advanced phases of the pathology (Björklund and Koivunen, 2005). In the early phases MMP-2 is crucial in creating a favorable microenvironment for cancer cells growth and contributes to the epithelial-mesenchimal transition (EMT), as demonstrated for renal tubular epithelial cells and breast epithelial cells (Bourboulia and Stetler-Stevenson, 2010; Kim et al., 2011b, 2007). The acquisition of a mesenchimal phenotype is a hallmark of cancer cells, which lose their tissue-specific morphology in favor of a un-differentiated phenotype suitable for migration and invasiveness (Min et al., 2008; Tang et al., 2011). The transition requires the proteolytic shedding of adhesion molecules, such as cadherin and integrin, and a profound rearrangement of the cytoskeleton, which allows malignant cell to detach from the original tissue. These are all processes directly or indirectly linked to MMP-2 activity (Aresu et al., 2011; Ribeiro et al., 2010).

Tumor growth is sustained through multiple mechanisms. MMP-2 solubilizes TGF- $\beta$ by degrading decorin and is further able to process the inactive precursor of the cytokine (Imai et al., 1997; Yu and Stamenkovic, 2000); TGF- $\beta$, while carrying homeostatic and pro-apoptotic stimuli in physiological condition, is a positive regulator of tumor progression favoring a more invasive phenotype (Munger et al., 1999; Yu and Stamenkovic, 2000). In turn, TGF- $\beta$ induces MMP-2 gene transcription, creating a vicious cycle which definitely promotes tumor growth (Binker et al., 2011; Kim et al., 2007). MMP-2 activates a plethora of cytokines, growth factors and further inactivates adhesion molecules, as TNF- $\alpha$, MCP-3 (McQuibban et al., 2000), IGF, $\beta$-dystroglycan and FGFR (Fowlkes et al., 1994; Bozzi et al., 2009), modulating tumor cells metabolism, immune responses, receptor turnover (Levi et al., 1996) and cell resistance to apoptotis (Gondi et al., 2009). As matter of the fact, glioma cells treated with anti-MMP-2 siRNA trigger apoptosis (Badiga et al., 2011). IGF-BPs bind IGF, limiting its mitogenic activity: MMP-2 cleaves IGF-BPs enhancing IGF availability (Fowlkles et al., 1994). Further, MMP-2 inhibition stimulates autophagy-associated cell death in cancer cells (Jo et al., 2011).

The source of MMP-2 are malignant, stromal and immune cells which surround the neoplastic lesion (Coussens et al., 2000; Egeblad and Werb, 2002). The involvement of MMP-2 in the pathological tumorigenic process stems from its 
dysregulation due to release by malignant cells of molecules which trigger secretion of MMP-2 from their not-malignant neighbors (Himelstein et al., 1994; Du et al., 2008). As an example, we can refer to EMMPRIN, a class of glycoprotein secreted by tumor cells, which stimulates MMP-2 expression by fibroblasts (Biswas et al., 2010). Nonetheless, factors accounting for the unbalanced regulation of the enzyme are continuously identified; this is the case of the L1CAM adhesion molecule in a model of lung metastasis and of an E3 uniquitin ligase (c-Cbl) (Hauser et al., 2011; Lee and Tsygankov, 2010; Song et al., 2010).

The aberrant activity of MMP-2 originates also from the unbalanced activation of pro-MMP-2 (Sims et al., 2011); several evidences put the emphasis on the upstream over-expression or dys-regulated compartmentalization of MMP-2 activating enzymes (Sato and Takino, 2010). In this respect, novel in vivo mechanisms of pro-MMP-2 activation by macromolecules highly up-regulated in almost all tumors have been identified as for the HSP-90 and ATP-dependent activation the proMMP-2 at the cell membrane (Walsh et al., 2011).

The contribution of MMP-2 to invasiveness includes different aspects, namely (i) degradation of BM component (such as collagen IV, laminin and fibronectin) which assists cells migration to the bloodstream (Björklund and Koivunen, 2005), even though recent discoveries about amoeboid movement of cancer cells question the BM breakdown; (ii) release of bio-active factors; and (iii) exposition of hidden adhesion sites that drive cell motility (Reichel et al., 2008).

A precise spatial and chronological dys-regulation of MMP-2 is another important pre-requisite for efficient invasiveness (Stefanidakis and Koivunen, 2006). MMP-2 is often concentrated at the leading edge of migrating cells invadopodia in association with the vitronectin receptor alphaV beta3 integrin (Brooks et al., 1996). The complex occurs between the C-terminal hemopexin like domain of the enzyme and a integrin site distinct from the RGD binding site (Stetler-Stevenson, 1999). This feature allows the cells to keep unaltered the ability to adhere to ECM molecules, taking in the same time advantage of a highly targeted proteolytical activity.

4.1.3.3. Angiogenesis. MMP-2 contribution to angiogenesis is supported by evidences mainly on MMP-2 knock out mice (see Table 6), which show deficient vascular architecture and altered angiogenesis in the microenvironment of cerebral tumors. Modulation of VEGF availability and pericytes homeostasis should represent key aspects (Badiga et al., 2011; Du et al., 2008; Itoh et al., 1999; Rojiani et al., 2010). Even though molecular mechanisms are still elusive, the previously mentioned compartmentalization of MMP-2 at the leading edge of invadopodia seems to be a pre-requisite for efficient angiogenesis, facilitating endothelial cell invasion and migration and, thus, the realization of the pre-metastatic niche (Brooks et al., 1996; Chetty et al., 2008, 2010; Erler et al., 2009).

On the other hand, it is widely known that by cleaving the collagen IV NC1 domain MMP-2 generates fragments with antitumoral properties, such as arrestin, canstatin and tumstatin, whereas cleavage of laminin-5 exposes cryptic pro-migratory binding sites (Giannelli et al., 1997).

\subsubsection{Non neoplastic diseases}

4.1.4.1. Disease of the cardiovascular system.

4.1.4.1.1. Atherosclerosis. Atherosclerosis is a pathological thickness of artery wall due to fatty acids and cholesterol deposition (Caceres et al., 2010). An early event in the onset of the disease is the altered permeability of the endothelial layer to LDL and fatty acids which then are loaded in the sub-endothelial space (Hansson, 2005). The chemical modification of these macromolecules, such as oxidization, triggers inflammation with recruitment of monocytes/macrophages which invade the atherosclerotic lesion (Libby, 2002). Foam cells, a peculiar histological marker of the disease, are macrophages which uptake oxidized LDL (Shashkin et al., 2005). In the advanced stage, a fibrous cap, made up by smooth muscle cells and ECM components, surrounds the lesion: its rupture generally triggers the thrombus-mediated life-threatening acute event.

Gelatinase A is generally constitutively expressed in almost all human tissues and is weakly inducible. Anyway, in the inflammatory context of atherosclerotic lesion the enzyme is particularly abundant.

Experimental models on mice have highlighted its role in atherosclerosis progression, whereas epidemiological investigations in patients reveal that genetic variants of MMP-2 gene and in particular of the promoter region facilitate the onset of the disease (Fiotti et al., 2010; Volcik et al., 2010; Wågsäter et al., 2011).

MMP-2 expression strongly correlates with plaque rupture, by both degrading components of the fibrous cap and modulating the bioavailability of cytokines that contribute to plaque instability, as in the case of TNF- $\alpha$ (Heo et al., 2011; Kaneko et al., 2011).

Furthermore, MMP-2 plays a pivotal role in smooth muscle cells migration, an event which occurs at an advanced stage of the disease and has a strong impact on the formation of the fibrous cap itself (Butoi Dragomir et al., 2011; Jung et al., 2010). Nonetheless, MMP-2 source is just represented by these cells which secrete the enzyme in response to stimuli present in the atherosclerotic lesion (Kodali et al., 2006). As an example, several factors, such as thrombospondin and angiotensin, which promote atherosclerosis progression positively modulate MMP-2 expression by smooth muscle cells. ROS and oxidized LDL further induce gelatinase A secretion (Lee et al., 2003; Luchtefeld et al., 2005).

Notably, oxidized LDL can modify metabolisms and the proteomical pattern of cells surrounding atherosclerotic lesion and should further contribute to the epigenetic modification of MMP-2 gene which ultimately lead to up-regulation of the enzyme (Chen et al., 2011a). 
4.1.4.1.2. Stroke. In the central nervous system MMP-2 is normally expressed by neurons, microglia and astrocytes and presumably drives physiological remodeling of BBB (Del Zoppo et al., 2007). It is worth pointing out that MMP-2 is up-regulated within $1 \mathrm{~h}$ after stroke (Del Zoppo et al., 2007) and that local hyperthermia and flogistic stimuli enhance the MMP-2 content. This condition leads to an increased degradation of basement membrane components, in particular collagen IV and laminin (Alam et al., 2011). Moreover, MMP-2 should contribute to the activation of glial cells, facilitating the consequent immune modulation that sustains flogosis (Nakaji et al., 2006).

The correlation of MMP-2 with stroke is also reinforced by genetic evidences, namely (i) specific MMP-2 variants correlate with stroke and disease outcome (Manso et al., 2010), (ii) the enhanced toxicity for endothelial cells and the increased risk of BBB breakdown observed in some patients can originate from the MMP-2 dependent proteolytical shedding of a toxic soluble variant of $A \beta$-peptide (Hernandez-Guillamon et al., 2010).

4.1.4.1.3. Myocardial infarction. The outcome of myocardial infarction (MI) closely depends on a adequate ventricular architecture remodeling. The process is mainly driven by the deposition of ECM macromolecules in a tightly regulated manner in order to prevent stiffness and/or other pathological conditions that often impairs myocardial viscoelasticity ending up with a heart failure (Mountain et al., 2007). The role of MMP-2 in cardiac tissue encompasses critical steps in heart and cardiovascular system development during embryogenesis: nonetheless, the enzyme is well represented in pathological conditions that require ECM remodeling and restoration of cardiomyocytes homeostasis (Linask et al., 2005).

MMP-2 is a key player in the recovery phases after MI: its appearance follows a defined pattern, being maximally induced within the first 3 days (Secchiero et al., 2010). MMP-2 is mainly secreted by endothelial cells in response to cytokines and EMMPRIN, which are the typical inflammatory stimuli in the infarcted myocardium (Nie et al., 2009). IL-1 $\beta$ should be the main MMP-2 inducer in the post-infarction area, but also TNF- $\alpha$ has a major relevance (Bujak et al., 2008; Herrmann et al., 2011; Zitta et al., 2010).

The activity of MMP-2 deals with two different aspects. It is necessary to prevent fibrosis, promoting ECM molecules breakdown and this aspect is further reinforced by the evidence that mesenchimal stem cells stimulate secretion of MMP-2 and delay fibrosis (Giricz et al., 2006; Mias et al., 2009). However, recently reported evidence indicates that MMP-2 activity could trigger the pathological tenascin-C deposition upon fibronectin degradation, suggesting that a MMP-2 inhibition therapeutical strategy should improve the MI outcome (Ma et al., 2011).

4.1.4.2. Diseases of the nervous system. Multiple sclerosis is characterized, from an histological point of view, by focal lesions with a dense infiltrate of macrophages and lymphocytes which massively attack myelin of brain and spinal cord axons. Therefore, the disease is determined by progressive and disseminated destruction of myelin layer.

The involvement of MMP-2 on these pathologies is not particularly significant, at least on the basis of the up to now reported evidences.

Experimental findings illustrate an elevated concentration of MMP-2 in serum of patients with MS, SLE, and a putative correlation between SNPs in MMP-2 gene and disease onset, but the molecular rationale is mys-leading even though myelin basic protein indeed can be in vitro degraded by MMP-2 and early axonal injury might be induced by the enzyme (Avolio et al., 2003; Benesova et al., 2008, 2009; Chang et al., 2008; Comabella et al., 2009; Diaz-Sanchez et al., 2006; Fainardi et al., 2009; Shiryaev et al., 2009a).

4.1.4.3. Diseases of joints and muscular system. Rheumatoid arthritis is a chronic muscolo-skeletal disease of the synovial lining. The progress of the disease after the antigenic stimulus evolves through an acute inflammation which rapidly becomes chronic: the consequent proliferative erosive synovitis brings about bone and cartilage destruction inducing severe pain and inability.

Diagnostic markers of the disease are the rheumatoid factor and anti-citrullinated antibodies: the first one is an antibody that binds the Fc portion of the IgG, promoting the formation of immune-complexes, whereas anti-citrulline antibodies recognize proteins which expose the post-translationally modified aminoacidic residue (Van Vollenhoven, 2011).

The inflammatory condition leads to the formation of a "pannus", an aberrant layer of fibrotic or granulated tissue deposited by fibroblasts and immune cells lining the lesion. The "pannus", in turn, stimulates the expression and secretion of proinflammatory cytokines, such as IL-1 $\beta$, TNF- $\alpha$ and MCP- 1 by chondrocytes, macrophages and other cells type that complete the vicious cycle by triggering the expression of several bone and cartilage-degrading proteases (Wang et al., 2010a). Hence, many enzymes are over-represented in this context including MMP-2 and MMP-9 (see below), whose high serum concentrations are commonly detectable in patients with RA (Chang et al., 2008; Kim et al., 2011b; Yang et al., 2008).

MMP-2 contribution to rheumatoid arthritis is illustrated by several experimental findings on animal models and gets stronger evidence from the elevated levels in the serum and in the synovial fluid of patients at an advanced stage of the disease (Chang et al., 2008; Goldbach-Mansky et al., 2000). Even though MMP-2 is a matrix degrading enzyme and ECM remodeling is a key pathological event in the onset and progression of RA, many evidences concur in defining a suppressive role of the enzyme, consistently with the notion that it displays a systemic pro-homeostatic function.

The idea of a suppressive role for MMP-2 originates from animal models: the most relevant evidence comes from genetically modified MMP- $2^{-1-}$ and MMP-9 $9^{-1-}$ mice where the gelatinase A ablation results in a dramatic increase of circulating autoantibodies (Itoh et al., 2002). 
Subsequently, other experimental findings have casted light on a differential regulation of gelatinases in the progression of the disease, which reinforce the hypothesis of the elevated concentration of MMP-2 as a consequence of the effort in down-regulating the inflammatory stimulus.

However, the involvement of MMP-2 in the progression of the disease cannot be ruled out, since it is otherwise evident that MMP-2 up-regulation stimulated by TLRs pathways in synovial tissue modulates endothelial cells migration and new vessel formation, a pre-requisite for subsequent flogosis onset and joint destruction while the ECM degrading activity further promotes cartilage and bone breakdown (Saber et al., 2011; Sacre et al., 2007). Elevated levels of the enzyme are determined by cytokines stimulation of macrophages and, mainly, of synovial fibroblasts, the main source of the enzyme.

MMP-2 expression is triggered by macrophages migration inhibitory factor and other cytokines or soluble factors, such as IL-1 $\beta$ and cyclophillin A, which stimulate the expression of the glycoprotein EMMPRIN (CD147) on the cell surface of macrophages, thus inducing MMPs expression (Ahmed et al., 2006; Pakozdi et al., 2006; Zhou et al., 2008; Zhu et al., 2006).

Nonetheless, the ability of MMP-2, once secreted, to degrade and inactivate cyto- and chemokines may account for its protective role in the molecular basis of RA aetiology.

\subsection{MMP-9}

\subsubsection{Structural peculiarities}

MMP-9 (gelatinase B) (92-85-82 kDa for the pro-enzyme, the intermediate form and the active enzyme, respectively) domain composition has an high homology degree with that of the related MMP-2 with the exception of a type V collagen-like extensively O-glycosylated linker domain (see Fig. 1) (OG-domain) (Elkins et al., 2002). Insights on MMP-9 structure come out from resolution of mutants lacking the hemopexin and the type $\mathrm{V}$ collagen domains since their presence impairs crystals formation. Anyway, overall MMP-9 structure has been deduced using atomic force microscopy combined with small angle Xrays scattering and analytical ultracentrifugation (Rosenblum et al., 2007a).

The catalytic domains of MMP-2 and MMP-9 share the same structural organization; however, the S1 site is particularly deep in MMP-9 and the main difference with the gelatinase A concerns the presence of a Asp410 instead of a Glu in the S2 subsite (Chen et al., 2003).

The Collagen Binding Domain (CBD) is located at one terminus of the catalytic domain and it shares with MMP-2 the presence of conserved cysteines and aromatic residues which limit domain flexibility. The CBD of MMP-9 has a different orientation with respect to the catalytic site and no interaction occurs between these domains (Rosenblum et al., 2007b).

The hallmark of MMP-9 structure is the unusually long linker domain (54 residues) which displays a particularly high content in Ser and Thr residues with a variable pattern of O and N-glycosylation (Van den Steen et al., 2002). The domain is inserted between the catalytic and the hemopexin-like domain, rendering very unlikely their tight interaction, and confers an extraordinary flexibility to the enzyme (Rosenblum et al., 2007a; Overall and Butler, 2007). This feature has suggested the intriguing "inchworm model" of MMP-9 movement along collagen fibrils, which furnish an explanation for those still obscure aspects concerning MMPs interaction with large ECM substrate (Overall and Butler, 2007). The OG domain further participates in the MMP-9 dimerization and regulates the trafficking in the intracellular compartments and the secretion (Van den Steen et al., 2006).

The hemopexin-like domain carries out substrate binding functions together with the CBD. Furthermore PEX domain should be involved in MMP-9 homodimerization. Differently from MMP-2, MMP-9 physiologically exists as monomer, dimers or associated to the transport protein lipocalin (Van den Steen et al., 2002): each conformation is generally represented in tissues and they can be released by the same cell type in association to a specific MMP-9 biological activity (Bolignano et al., 2010; Dufour et al., 2010). As for MMP-2, the hemopexin-like domain can be targeted by TIMPs and in particular by TIMP-1 and TIMP-4.

The pro-domain has the classic $\alpha$-helix composition and the Cys-99 of the conserved cysteine switch motif is the fourth ligand of the $\mathrm{Zn}^{2+}$ atom in the enzymatic pro-form. Displacement of the pro-peptide is in vivo exerted by many soluble proteases, mostly by MMP-3, MMP-2, MMP-7, MMP-13 and other soluble proteases, such as kallikrein or trypsin or uPA (Van den Steen et al., 2002). Studies on the stepwise activation of pro-MMP-9 by kallikrein have recently suggested a novel mechanism which replaces the cysteine-switch dogma based on the mere degradation of the propeptide domain. The model proposes that the dissociation of Cys-99 from the $\mathrm{Zn}^{2+}$ atom is early driven by protein-protein interaction, while the proteolytical cleavage of the propeptide occurs only at a later stage (Kotra et al., 2001; Rosenblum et al., 2007b).

\subsubsection{Biological aspects}

Substrate specificity of MMP-9 clearly overlaps with that of MMP-2, even though with different affinities (see Table 3): basement membrane components are mainly targeted, but cytokines, growth factors, receptors are highly represented (Björklund and Koivunen, 2005) (see Table 3). MMP-9 is physiologically expressed by a limited cohort of cell types, mainly immune cells, and it is highly inducible (Van den Steen et al., 2002). Therefore its distribution is spatially and chronologically defined, making it a prototypical target in inflammatory events: hence, the proteolytical breakdown of substrates often occurs in association to anti-homeostatic conditions (Van den Steen et al., 2002). Consistently with the identification of cis-acting promoter elements, transcriptional activation of MMP-9 is regulated by a multitude of factors which render highly complex the plethora of stimuli that finely tune its biological activity (Lauricella-Lefebvre et al., 1993; Munaut et al., 1999). MMP-9 is induced by adhesion molecules, mainly activated integrins, cytokines and growth factors (Beliveau et al., 
2010; Zhao and Benveniste, 2008). As an example, cell binding to vitronectin or fibronectin trigger MMP-9 expression, IL-1 $\beta$, TNF- $\alpha$ or TGF- $\beta$ induce MMP-9 in a autocrine and paracrine fashion, EGF release or the aberrant activation of the EGFR result in over-expression of MMP-9 (Coulson-Thomas et al., 2010; Ehrenfeld et al., 2011; Ikari et al., 2011; Lin et al., 2009; Yu and Stamenkovic, 2000).

Several molecular pathways, once activated, regulate MMP-9 expression: MMP-9 is controlled by the Raf/MEK/ERK cascade and the transcription factors SLUG and NF- $\kappa B$ induce MMP-9 expression (Beliveau et al., 2010; Sun et al., 2008; Moon et al., 2007). Furthermore, the transcriptional stability is maintained through the p38 MAPK/MAPK2 signaling and, interestingly tissue transglutaminase 2 is reported to promote MMP-9 expression (Cai et al., 2011; Kumar et al., 2010; Li et al., 2011).

The homeostasis of the vast majority of the previously mentioned factors that induce MMP-9 expression is compromised in malignancies; as a matter of the fact, MMP-9 is over-represented in almost all human cancer (Egeblad and Werb, 2002).

MMP-9 involvement is further described for cardiovascular, respiratory and auto-immune diseases (e.g., Systemic Lupus Erythematosus, Sjögren's syndrome, Multiple Sclerosis, Rheumatoid Arthritis, polymyositis, asthma, chronic obstructive pulmonary disease, $C$ protein-induced myocarditis, lupus nephritis, auto-immune inner ear disease and bullosus pemphigoid) (Du et al., 2008; Fenton et al., 2009; Hulkkonen et al., 2004; Ito et al., 2009; Ma et al., 2010; Matsumoto et al., 2009; Noseworthy et al., 2000; Pathak et al., 2011). In some cases, the molecular mechanisms underlying its pathogenic activity have been described in detail: therefore, the discussion of MMP-9 role in human pathologies will be limited to those more represented in literature (see Table 4).

\subsubsection{Neoplastic diseases}

4.2.3.1. Epidemiological aspects. MMP-9 and tumorigenesis is a long standing association which have extensively stimulated research over the past decades (Björklund and Koivunen, 2005; Deryugina and Quigley, 2006). Experimental models, mostly based on MMP-9 knock out mice, have provided unequivocable evidences about the pivotal role played by the enzyme in tumor growth, invasiveness and angiogenesis (Egeblad and Werb, 2002; Fingleton, 2006; Itoh et al., 1999; Wielockx et al., 2001): nonetheless, there is compelling evidence that biology of the enzyme is otherwise complex and a final convincing picture is far from being formulated. In this respect, many findings envisage anti-tumoral properties for MMP-9, as in the case of breast cancer regression in a MMP-9 deficient mouse model (Bendrik et al., 2008). Such a complex biology of the enzyme is further highlighted by the description of a anti-proliferative effects in prostate cancer cells and in colitis associated cancer (Biswas et al., 2010; Garg et al., 2010).

In accordance to the tissue-specific distribution of the enzyme, MMP-9 level in serum is almost undetectable under physiological conditions: therefore, the diagnostic and prognostic value of MMP-9 quantification appears even more significant (Turpeenniemi-Hujanen, 2005). A large number of epidemiological studies illustrate that MMP-9 concentration is abnormal in serum from patients with melanoma, gastric cancer, breast cancer, bladder cancer, colorectal cancer, brain cancer, pancreatic cancer and prostate (Chu et al., in press; Daniele et al., 2010; Jensen et al., 2010; Joergensen et al., 2010; Littlepage et al., 2010; Liu et al., 2010; Nikkola et al., 2005; Offersen et al., 2010; Ranogajec et al., in press; Buhmeida et al., 2009). Elevated MMP-9 concentrations are generally associated to poor prognosis.

4.2.3.2. Tumorigenesis. MMP-9 has been historically identified as a basement membrane degrading protease, due to its high affinity for collagen IV. Hence, pathogenicity had been initially limited to tumor invasiveness: it was anyway clear that MMP9 contribution was much broader. Matrisian and colleagues have first demonstrated that tumor cells over-expressing TIMP1 , once injected in the chorioallantoic membrane of chicken, invade the host tissue but fail to proliferate: this statement clearly indicated that the biological role of MMPs, and in particular of MMP-9 which is preferentially targeted by TIMP-1, was not strictly dependent on cancer cell invasion, but rather in tumor growth (Chambers and Matrisian, 1997; Khokha et al., 1992). MMP-9 was then shown to modulate the bioavailability of growth factors and to disrupt cell-cell contacts, dramatically affecting cell proliferation and survival. In this respect, the MMP-9 dependent release of TGF- $\beta$ is a key aspect (Yu and Stamenkovic, 2000); similarly, MMP-9 shedding of the membrane component of HB-EGF lead to increased concentration of the soluble EGF. Both these factors display positive properties for tumor growth and contribute to the epithelial-mesenchimal transition (Aresu et al., 2011; Ongusaha et al., 2004; Wu et al., 2011a). MMP-9 cleaves nexin-1 and adhesion molecules, such as the $\beta$-subunit of dystroglycan (Xu et al., 2010; Bozzi et al., 2009), enhancing tumor detachment from the primary site and tumor invasiveness. Furthermore, MMP-9 degrades collagen IV and other basement membrane components and exposes cryptic binding sites for cell migration or releases hidden bio-active factors (Schenk and Quaranta, 2003; Stetler-Stevenson and $\mathrm{Yu}, 2001$ ). Nonetheless, progress in MMP-9 research are currently revealing that MMP-9 biology is even more complex and that its pathogenic activity takes advantage of many undefined aspects, including interaction with enzymes which share the same tissue localization (Gioia et al., 2009). Interestingly, the MMP-9 source are not exclusively cancer cells, but also inflammatory cells (Coussens et al., 2000; Egeblad and Werb, 2002). In particular, polymorphonucleated cells (PMN), which store elevated concentrations of MMP-9 in their mature granules, provide a consistent reservoir of MMP-9 (Ardi et al., 2007). This statement is further reinforced by the evidence that PMN secrete MMP-9 devoid of TIMP-1, which can be readily activated (Egeblad and Werb, 2002; Du et al., 2008); on the other hand, fibroblasts and endothelial contribute to a much lesser extent.

Attention has recently focussed on the definition of the "tumor niche", a specific anatomical microenvironment adapt for cancer cells growth (Coulson-Thomas et al., 2010; Kaplan et al., 2006). In this context, MMP-9 contributes by controlling cell division and by conferring resistance to apoptosis and to immune surveillance. Mitotic phases are accompanied by a highly 
conserved and dynamic distribution of the enzyme as from studies in neuroblastoma cells and in macrophages (Sans-Fons et al., 2010; Ganji et al., 2011). Furthermore, MMP-9 affects apoptosis by degrading apoptotic molecules, as the Kit-ligand, hence promoting cells survival (Meyer et al., 2005). In addition, MMP-9 shedding of ICAM-1 confers protection to NK and Tcell mediated cytotoxicity (Fiore et al., 2002).

Another aspect carefully examined concerns the MMP-9 compartmentalization, which further implies intriguing therapeutical strategies (Ezhiralasan et al., 2009). MMP-9 activity is highly compartmentalized in invadopodia, the leading edge of invading cells. The enzyme interacts with trans-membrane glycoprotein, such as the heparan sulfate proteoglycan (CD44) and integrins (Yu and Stamenkovic, 2000). It is important to outline that such a localization is not a specific cancer cell property, since it is widely observed also in endothelial and immune cells (Mäkelä et al., 1998; Partridge et al., 1997); it is otherwise evident that tumor cells fail to balance this process (Björklund and Koivunen, 2005). The association of MMP-9 and CD44, whose cell membrane distribution is diffusely dys-regulated in cancer, promote growth and survival by optimizing the TGF- $\beta$ activation (Bourguignon et al., 1998).

The interaction between integrin and MMP-9 deserves a more extended comment: it has been previously reported that integrin activation induces MMP-9 expression upon binding to those ECM macromolecules displaying the RGD sequence. MMP-9 in turn can readily activate integrin promoting cell motility. Invadopodia of tumor cells show strong MMP-9 gelatinolytic activity and down-regulation of MMP-9 expression results in decreased invasion and decreased integrin expression on the cell surface (Lagarrigue et al., 2010; Veeravalli et al., 2010).

It is now evident that these molecules interact through the C-terminal hemopexin-like domain of the enzyme: this aspect is a challenging task in the comprehension of the molecular mechanisms underlying MMP-9 activity (Radjabi et al., 2008). The precise localization allows the cells to help proteolysis; thus, integrins do not lose their ability in recognizing its ECM partner, even when MMP-9 is bound, and MMP-9 might provide the helping hand in the amoeboid movement exposing the hidden binding sites, since the RGD sequence is often cryptic. Additionally, the hemopexin-like domain alone can act as a functional ligand for $\alpha_{\mathrm{iv}} \beta_{1}$ integrin, inducing intracellular signals which promote cell survival (Redondo-Muñoz et al., 2008). The existence of intracellular signals triggered upon binding of MMP-9 hemopexin-like domain with receptors is further highlighted by evidences on leukemia B cell survival (Muñoz et al., 2010). The administration of the hemopexin-like domain has been confirmed to disrupt angiogenesis and to delay the growth of glioblastoma in xenograft mice (Ezhilarasan et al., 2009).

4.2.3.3. Angiogenesis. Beside the involvement in tumor growth and in invasiveness, as above discussed, MMP-9 exclusively contributes to a critical step of tumorigenesis: the so called "angiogenic switch". The first evidence was obtained in the Rip1-Tag2 Mice (Bergers et al., 2000). Vascular endothelial growth factor (VEGF), the main stimulus for endothelial cells activation and for new vessel formation, is normally sequestered in the ECM (Ferrara et al., 2003). MMP-9 was identified as the main enzyme involved in VEGF release from the natural reservoir. In the last decade the role of MMP-9 in angiogenesis has represented an important matter of debate: experimental models both in vivo and in vitro have extended the original finding and are now revealing that the enzyme contribute is complex (Ahn and Brown, 2008). Even before originating a metastasis, tumor cells implanted in a mouse model attract VEGF receptor positive pro-inflammatory cells that create a niche wherein tumor cells can proliferate (Kaplan et al., 2005). MMP-9 secreted by these immune cells, by degrading basement membrane and by releasing VEGF and Kit-ligand promote recruitment of a large number of inflammatory cells that ultimately build up the niche and sustain pericytes correct positioning on newly formed blood vessels (Chantrain et al., 2004; Kaplan et al., 2005; Karroum et al., 2010; Zijlstra et al., 2006). Notably, MMP-9 modulates also vasculogenesis, helping the implant of stem cells progenitor of endothelial cells in tumor vasculature (Hiratsuka et al., 2006; Shojaei et al., 2007).

\subsubsection{Non neoplastic diseases}

4.2.4.1. Disease of the cardiovascular system.

4.2.4.1.1. Atherosclerosis. MMP-9 is over-represented in sera from patients with advanced lesions so that, even in this case, its quantification can be considered a suitable marker for diagnosis and treatment (Jefferis et al., 2010). Furthermore, from a clinical point of view, fragments of collagen III generated by MMP-9 are currently under investigations for their putative diagnostic relevance (Barascuk et al., 2011; Zeng et al., 2005).

Stimuli, such as b-FGF, TGF- $\beta$ and IL-1 $\beta$ and other soluble cytokines as well as oxidized compounds, indeed trigger MMP-9 expression (Chen et al., in press, 2011a,b; Seizer et al., 2010), whereas conditions that reduce secretion of these pro-inflammatory cytokines affects the MMP-9 content as well (Bhaskar et al., 2011; Sigala et al., 2010). Reactions by-products in the inflammatory context further induce the aberrant activation of proMMP-9 (Bonacci et al., 2011). Notably, neutrophils are not recruited in lesions, hence MMP-9 main source are presumably macrophages (Lusis, 2000).

MMP-9 plays a key role in facilitating the migration of smooth muscle cells into the lesion and then it is presumed to contribute to lesion expansion and fibrous cap formation (Galis et al., 2002; Wågsäter et al., 2011).

The MMP-9 mediated degradation of elastin and other components of the fibrous cap lead to its rupture (Gough et al., 2006; Rodriguez-Feo et al., 2008). Even though the strict association between elevated levels of MMP-9 and increased risk of acute event such as ischemia or stroke has been proved, a pro-fibrotic role of MMP-9 in plaque formation has been further described (Lemaitre et al., 2009), which is exerted through an elevated deposition of collagen more than a direct involvement in plaque rupture. 
Anyway, the evidence that pharmacological targeting of MMP-9 correlates with plaque stability and reduced incidence of coronary events highlight the role of the enzyme in the pathogenesis of atherosclerosis (Moon et al., 2007; Yamamoto and Takai, 2009).

4.2.4.1.2. Stroke. As previously discussed, the consequence of an atherosclerotic plaque rupture is the thrombus-dependent impaired perfusion of heart and/or brain. The MMP-9 serum concentration is elevated in patients with acute ischemic events and this generally correlates with a poor prognosis, being strictly associated to edema formation (Ramos-Fernandez et al., 2011). In the acute event, microglia cells are stimulated by released pro-inflammatory factors to secrete large amounts of MMP-9, which the contributes to the blood-brain barrier (BBB) breakdown (Chen et al., 2009; Copin et al., 2011; Del Zoppo et al., 2007). Occludin, the main component of astrocytes tight junctions, is targeted by the pathogenic activity of MMP-9 and this event usually ends up in alterations of BBB microvascular permeability (Liu et al., 2009). Interestingly, in a mouse experimental model, the gene therapy to inhibit MMP-9 improves the outcome when administered after acute events (Hu et al., 2010; Tu et al., 2011).

4.2.4.1.3. Myocardial Infarction. The recent identification of elevated levels in serum from patients with myocardial infarction (MI) has opened the perspective of a MMP-9 involvement not merely in the atherosclerotic plaque instability but also in the recovery phases (see also MMP-2) (Zeng et al., 2005). The dys-regulation of the enzyme further stems from the unbalanced MMP-9/TIMP-1 ratio and is generally associated with poor prognosis (Cao et al., 2011; Ghaderian et al., 2010; Hansson et al., 2011).

Two chronologically distinct events lead to MMP-9 increase in the infarctuated tissue: the first one corresponds to the neutrophils recruitment, while the second one, which occurs several days afterwards, is linked to macrophages and lymphocytes crowding in the flogistic microenvironment (Mukherjee et al., in press). A benign clinical outcome of MI strictly depends on the adequate remodeling of ventricular architecture: however, correct myocardial regeneration is often impaired by tenascin-C deposition which directly correlates with the the upstream degradation of fibronectin, readily secreted by fibroblasts and myo-fibroblasts. Fibronectin degradation by MMP-9 leads to the release of fragments unable to inhibit tenascin C accumulation (Ma et al., 2011). Therefore, elevated concentrations of MMP-9 favor the unbalanced distribution of these ECM components, leading to compromised ventricular architecture.

Interestingly, transplantation of mesenchymal stem cells down-regulates the expression of MMPs in fibroblast and this is accompanied by an improved outcome (Wang et al., in press), with ameliorated fibrosis and attenuated inflammation (Oyamada et al., 2011).

In conclusion, it is now recognized that the MMP-9 concentration in MI is a delicate equilibrium as its inhibition prevents ventricular stiffness and ECM remodeling, though impairing the otherwise necessary angiogenesis (Cleutjens and Creemers, 2002; Heymans et al., 1999, 2005; Lindsey et al., 2006).

\subsubsection{Diseases of the nervous system.}

4.2.4.2.1. Multiple sclerosis. Among proteases taking part into the disease onset and progression, MMP-9 has a 3-fold role, since it contributes to (i) the blood-brain barrier breakdown (BBB), (ii) the generation of auto-immune epitopes, and (iii) the modulation of cytokines bio-availability.

Epidemiologic investigations correlate MMP-9 concentration in serum with advanced stages of the disease, hence its quantification could represent an intriguing prognostic factor (Benesova et al., 2009; Glass-Marmor et al., 2009; Rosenberg, 2005). Moreover, studies on gene polymorphism reveal that SNPs on MMP-9 gene can facilitate its involvement in MS (Benesova et al., 2008; Fernandes et al., 2009; Garcia-Montojo et al., 2010; La Russa et al., 2010).

The molecular mechanisms of MMP-9 pathological activity have been deduced mostly through the highly related experimental autoimmune encephalomyelitis (EAE) in mouse model: thus, MMP-9 knock out confers protection against the onset of the disease (Dubois et al., 1999; Opdenakker et al., 2006; Du et al., 2010).

Gelatinase B is mainly expressed by immune cells. The need of cytokine-dependent inducibility of the MMP-9 gene promoter fits well with the dynamic ability of these cells to respond, in a spatially and chronologically defined manner, to autocrine and paracrine stimuli (Van den Steen et al., 2002): therefore, in a context of inflated flogosis, as observed in multiple sclerosis lesions, MMP-9 concentration is predictably aberrant. Nonetheless, therapeutical approaches, such as the administration of IFN- $\beta$ and quercetin, decrease the MMP-9 expression in monocytes isolated from patients, lowering the MMP-9/ TIMP-1 ratio (Bernal et al., 2009; Sternberg et al., 2008; Yen et al., 2010).

A plethora of stimuli concurs to the MMP-9 aberrant activation, namely (a) EMMPRIN, a glycoprotein dys-regulated in the pathology, which promotes secretion from resident or activated microglia cells (Agrawal et al., 2011; Fabriek et al., 2007; Shinto et al., 2011); (b) apoptotic neurons, which secrete MMP-3, the main pro-MMP-9 activator (Kim and Lee, 2005); (c) fibronectin and vitronectin of the basement membrane, which stimulate MMP-9 synthesis by activated microglia cells in a integrin-dependent manner (Milner et al., 2007).

There is compelling evidence that MMP-9 pathogenic activity further includes the modulation of cross-talk between immune system compartments (which hinders remission) and, intriguingly, the generation of auto-immune epitopes. About this last aspect, two proteins have been identified as the main source of auto-immunoreactivity: the small heat shock protein $\alpha$-B crystallin and the myelin basic protein. They are both degraded by MMP-9 (Shiryaev et al., 2009a; Van Noort and Amor, 1998) and fragments share the immunogenic property (Milward et al., 2008; Shiryaev et al., 2009a). Additionally, the therapeutical administration of not-glycosylated interferon- $\beta$ has been demonstrated to be detrimental for disease outcome, 
since the MMP-9 dependent cleavage of that cytokine form, initially adopted in clinical trials, has been shown to be a further source of auto-antibodies (Nelissen et al., 2003).

As a matter of the fact, the combination of therapies addressed toward a suppression of the immune system activation and the inhibition of MMP-9 activity are promising target for long-term remission of the pathology (Muroski et al., 2008).

4.2.4.2.2. Alzheimer's diseases. Alzheimer's disease is a neurodegenerative process characterized by senile plaques deposition and neurofibrillary tangles. The $\beta$-amyloid peptide generated upon dys-regulated shedding of the APP precursor displays a marked tendency to aggregate forming unsoluble complex leading to neurons death (Finder, 2010).

The existence of an in vivo degradation of $\beta$-amyloid plaques has not been proven yet, but a vast deal of knowledge indicates that MMP-9 could bear this cyto-protective role. In fact, MMP-9 in vitro degrades $\beta$-amyloid aggregates (Yan et al., 2006). Several in vivo reported findings, mostly on animal models, reinforces this observation: increased levels of MMP-9 have been found in the cerebrospinal fluid and in post mortem AD brain; MMP-9 and TIMP- 1 co-localize with $\beta$-amyloid plaques (Asahina et al., 2001; Lorenzl et al., 2003).

In transgenic mice models (see Table 6), MMP-9 expression is further triggered by neurons upon exposure to A $\beta$ derived peptides and macrophages induces MMP-9 expression to get A $\beta$ clearance (Deb et al., 2003; Guo et al., 2006; Zhao et al., 2009).

Recently, it has been shown that astrocytes and neurons are stimulated by $A \beta$, in particular the $A \beta_{25-35}$ fragment, to secrete, respectively, MMP-9 and TIMP-1 (Hernandez-Guillamon et al., 2009). This is a relevant aspect since it is otherwise known that un-balanced MMP-9 activity affects viability of neurons (Doi et al., 2009; Gu et al., 2002).

4.2.4.3. Diseases of joints and muscular system. The physical and chemical properties of cartilage are conferred by extracellular matrix proteins, such as collagens. In particular, cartilage is made up by type II collagen: its destruction, as it occurs in RA, is followed by replacement with type I collagen whose different properties compromise joint anatomy. Collagen II is mainly degraded by the neutrophil-secreted MMP-8 and the cleavage takes place with the typical $1 /$ 4 and 3/4 pattern (see Section 3.1). MMP-9 does not efficiently cleave the whole molecule, but it displays a high affinity for the 3/4 fragment. The event ends up with the release of remnant epitopes which are the source of the anti-type II collagen auto-antibodies which can be found in RA lesions (Burrage et al., 2006; Van den Steen et al., 2004, 2005).

It is intuitive that gelatinase B contributes to the disease onset by modulating the bio-availability of these soluble factors, sustaining flogosis; as a matter of the fact, MMP-9 distribution in lesions correlates with that of IL-8, a cytokine which in turn triggers secretion of the enzyme from neutrophils (Pelletier et al., 2001).

In the last decade experimental evidences have suggested that the MMP-9 role could not be restricted to inflammation promotion, but should include the generation of remnant epitopes and/or the extracellular activation of ADAMTS-4, the main aggrecan-degrading protease (Nagase and Kashiwagi, 2003; Opdenakker et al., 2006; Van den Steen et al., 1998).

\subsubsection{Diseases of the respiratory system.}

4.2.4.4.1. Asthma. Asthma is a chronic condition characterized by airflow obstruction and bronchospasm triggered by mechanical, antigenic or microbial insult which leads to epithelial layer erosion and, in advanced phases, to injured tissue remodeling (Araujo et al., 2008; Kaminska et al., 2009).

The acute event is followed by deposition of several ECM macromolecules, mainly collagen III by fibroblasts, in airways sub-endothelial space. The balance between accumulation and degradation of these components can variably affect physicochemical properties of the airway. Several proteases take part into this phenomenon and MMP-9 is the most represented one in pulmonary fluids from patients with asthma (Atkinson and Senior, 2003). Under physiological conditions resident cells in lungs do not express constitutively MMP-9, but the asthmatic process induces its secretion by recruited neutrophils and its release by epithelial, endothelial, fibroblasts, smooth muscle cells and alveolar type II cells (Broide, 2008; Mehra et al., 2010; Sands, in press).

The MMP-9/TIMP-1 ratio is a crucial aspect in disease progression. In vitro and in vivo studies provide discordant findings: anyway, it appears clear that a too low MMP-9/TIMP-1 ratio results in airways wall thickness and impaired respiratory functions, whereas a too high in an excessive collagen III deposition in the sub-endothelial space and reduced elasticity (Matsumoto et al., 2005; Sands et al., 2009; Swartz et al., 2001; Todorova et al., 2010; Watson et al., 2010).

MMP-9 pathogenic activity is not limited to ECM remodeling: two main aspects deserve some comments. (i) Degradation of claudin and occludin destabilizes the tight junctions, determining epithelial permeability and increased cell anoikis (Vermeer et al., 2009); (ii) modulation of soluble factors bio-availability influence response by other cell types (see Table 3 ). Interestingly, activation of TGF- $\beta$, triggers the fibroblasts-mediated deposition of collagen III and further represents a stimulus for epithelial-mesenchimal transition which drives epithelial cells transformation in myofibroblasts, whose aberrant airways colonization represents an histological marker of chronic asthma (Doerner and Zuraw, 2009; Hoshino et al., 1998).

4.2.4.5. Systemic Lupus Erythematosus. Systemic Lupus Erythematosus is a type III auto-immunity reaction that potentially affects any district of the body, with largely variable clinical and serological manifestations caused by auto-antibodies and complement activation dependent on immune complexes (Hahn, 1998, 2003; Muñoz et al., 2010).

Markers of the disease are in particular represented by (i) auto-antibodies against nuclear proteins (anti-dsDNA) (Hahn, 1998), and (ii) impaired leukocytes apoptosis; this last evidence is accompanied by an insufficient clearance of apoptotic 
bodies which, indeed, act as a reservoir of autoantigen in the extracellular compartments (Denny et al., 2006; Emlen et al., 1994).

In the case of Systemic Lupus Erythematosus epigenetic factors seems involved in MMP-9 dys-regulation (see Table 4) since in $\mathrm{CD}^{+}$lymphocytes the MMP-9 gene is hypomethylated, reflecting a greater expression of the protease (Jeffries et al., 2011).

Even though MMP-9 proteolytical activity is a major determinant in the generation of auto-immune fragments, its contribution to the onset of the disease is still matter of debate.

Two evidences, reported by the same authors, illustrate the MMP-9 pathogenic activity (Cauwe et al., 2009, 2011). The first one is the identification of several intra-cellular novel substrates, which enlarge the enzyme degradome and could be involved in the development of such a pathology. In this sense, the impaired clearance of apoptotic bodies could represent a dramatic reservoir of autoantigens (Cauwe et al., 2008). On the other hand, recent findings in a mouse experimental model illustrate a protective role of MMP-9 on the onset and the progression of the auto-immune disorder (Cauwe et al., 2011). Mice MMP-9 knock out with a systemic auto-immune lymphoproliferative syndrome, develop a pathology several fold more aggressive than that of the MMP- $9^{+/+}$counterpart.

Therefore, the aetiology of SLE is still unclear, but the identification of MMP-9 activity seems to be a pre-requisite for a successful approach to the disease treatment.

4.2.4.6. Bullosus pemphigoid. Bullosus pemphigoid (BP) is an autoimmune skin blistering disease, where antibodies antihemidesmosome trans membrane protein B180 (collagen XVII) lead to inflammation and, finally, to dermis-epidermis separation (Kasperkiewicz et al., in press). Mast cells physiologically colonize the connective tissues and, upon activation by antigens and allergenic stimuli, they recruit leukocytes and other cell types which secrete proteases with ECM-degrading properties (Walker et al., in press). It is well known that MMP-9 synergizes with neutrophil elastases in the breakdown of almost all components of the extracellular matrix, a pre-requisite for the two-layers separation (Verraes et al., 2001).

The first evidence of the MMP-9 dominant role in disease progression comes from studies on MMP-9 deficient mice which do not develop BP even when anti-B180 autoantibodies are injected into the dermal-epidermal junction (Liu et al., 1998). Furthermore, elevated concentration of MMP-9 can be in vivo detected in serum and in blister fluid of patients (Niimi et al., 2006).

MMP-9 is readily released by neutrophils, but a major contribute comes from other cell types which secrete the enzyme in response to soluble stimuli, mainly IL-1 $\beta$ (Gounni-Abdelilah et al., 2006). The cytokine stimulates the expression of a not yet identified pro-MMP-9 activating protease, and the plasminogen/plasmin system may have a significant role (Liu et al., 2005). An additional mechanism could involve the recently identified Mast cell protease-4, which displays MMP-9 activating properties (Lin et al., 2011a).

\section{Stromelysins}

The class of stromelysins encompasses three enzymes, namely MMP-3 (stromelysin-1), MMP-10 (stromelysin-2), and MMP-11 (stromelysin-3).

The overall structure includes the catalytic domain, the hemopexin-like domain and the pro-peptide, but significant differences with other soluble MMPs are also found. In particular, MMP-11 exhibits an additional pro-protein convertase recognition sequence, which envisages a peculiar activation mechanisms (Visse and Nagase, 2003).

Although stromelysins have been discovered many years ago, there is a limited knowledge of MMP-10 and MMP-11 biology, whereas more convincing findings are reported for MMP-3, even though several aspects still remain obscure, mainly concerning its pathogenic activity.

Natural substrates have been identified and they are mostly represented by ECM matrix components (see Table 3): in particular, stromelysins share the ability to degrade (although with significantly different affinities among them) types IV and IX collagens, laminin, fibronectin, elastin, and proteoglycans. Additional substrates include cytokines, growth factors and regulatory soluble molecules (Visse and Nagase, 2003).

Each stromelysin has a different physiological distribution in human tissue, hence the types of processes which are modulated are largely variable.

Cancer is a pathology wherein a contribution of the three stromelysins has been described quite extensively, even though with divergent molecular mechanisms (Gill et al., 2004; McCawley et al., 2004; Wilkins-Port et al., 2009). Other pathologies, for which a contribution from at least of one member of this family has been reported, mainly include cardiovascular, degenerative and auto-immune diseases.

\subsection{MMP-3}

MMP-3 or stromelysin-1 was first described in 1985 as a 51-kDa protein secreted by rabbit fibroblasts.

Stromelysin-1 is synthesized as a pro-enzyme which is activated upon removal of the N-terminal pro-domain by serine proteases trypsin-2 (Moilanen et al., 2003) and matriptase (Jin et al., 2006), yielding a 43-kDa active enzyme. The active enzyme contains a catalytic domain and a C-terminal hemopexin-like domain presumably involved in the macromolecular 
substrate recognition (see Fig. 1). The catalytic core is very similar for the pro- and the active enzyme forms and it has a high homology degree with the same domain of fibroblast and neutrophil collagenases, as determined through NMR analysis (Becker et al., 1995; Zhang et al., 2000). The prodomain is a separate folding unit containing three $\alpha$-helices and an extended portion that lies in the active site of the enzyme.

\subsubsection{Biological aspects}

Matrix metalloproteinase-3 is expressed by a variety of cells, e.g. keratinocytes, fibroblasts and chondrocytes, and it is often released in association with MMP-10 by fibroblastic cells and by normal epithelial cells in culture and in vivo (Sternlicht et al., 1999; Visse and Nagase, 2003).

The main physiological role of MMP-3 is the activation of other proMMPs during extracellular matrix turnover (Brinckerhoff et al., 1990; Visse and Nagase, 2003). In fact, stromelysin-1 efficiently activates collagenases, matrilysin, and gelatinase $\mathrm{B}$; in particular, the action of MMP-3 on a partially processed pro-MMP-1 is critical for the generation of fully active MMP-1 (Imai et al., 1995; Murphy et al., 1992; Shapiro et al., 1995). The enzyme differs from collagenases by the inability to cleave type I collagen, anyway it degrades a wide range of ECM proteins, e.g., type IV, V, IX, and X collagens, proteoglycans, gelatin, fibronectin, laminin, and fibrillin-1 (Chin et al., 1985).

Furthermore, MMP-3 cleaves $\alpha 1$-proteinase inhibitor, tumor necrosis factor (TNF)- $\alpha$ precursor, myelin basic protein and interleukin (IL)-1 $\beta$, inactivating it (see Table 3). Moreover, MMP-3 activates TGF- $\alpha$ which then stimulates MMP-9 expression by macrophages in a COX-2 dependent way (Steenport et al., 2009).

MMP-3 expression is upregulated by interleukin-1 $\beta$ and downregulated by retinoic acid, dexamethasone and progesterone which inhibits also its secretion (Osteen et al., 2003; Saus et al., 1988).

\subsubsection{Neoplastic diseases}

The previously discussed role as proMMPs-activating enzyme indicates that the unbalanced MMPs, and in particular MMP-9, distribution in solid tumors should be related to MMP-3 enhanced secretion. Nonetheless, although findings on skin tumors and the documented MMP-3-dependent release of angiostatin-like fragments from plasminogen suggest an anti-tumoral activity, a vast deal of knowledge concurs in defining a major role for MMP-3 in tumor progression and angiogenesis (Lijnen et al., 1998; McCawley et al., 2004).

As an example, phenotypically normal mammary epithelial cells in vivo form epithelial glandular structures in absence of stromelysin-1, whereas they transform into invasive mesenchymal-like tumors in the presence of the enzyme (Thomasset et al., 1998). Once initiated, tumor progression is not linked to MMP-3 expression anymore. Premalignant changes and malignant conversion in mammary glands regulated by MMP-3 have been reported in transgenic mice and, interestingly, the co-expression of TIMP1 reverts this process (Sternlicht et al., 2000). These findings clearly indicate that MMP-3 influences tumor initiation and alters the neoplastic risk (Sternlicht et al., 1999).

The MMP-3 pathogenic activity results in the modulation of growth factors and of cytokine availability which affect both tumor growth per se and neutrophils and macrophages recruitment that often provide a consistent reservoir of pro-angiogenic molecules. Noteworthy, TGF- $\beta$ bio-availability is strictly linked to MMP-3 activity and such an association further strengthens the recent suggestion of a MMP-3 involvement in the epithelial-mesenchimal transition (Blavier et al., 2010; Lochter et al., 1997; Maeda et al., 2002).

Another relevant aspect is the ability of MMPs to target substrates that modulate apoptosis of cancer cells, even though molecular mechanisms are still elusive. Anyway, it is known that MMP-3 exerts a pro-apoptotic action on epithelial cells and MMP-3-deficient mice have a lower degree of TNF- $\alpha$-induced of apoptosis (Wielockx et al., 2001; Witty et al., 1995).

\subsubsection{Non-neoplastic diseases}

\subsubsection{Diseases of cardiovascular system.}

5.1.3.1.1. Atherosclerosis. The primary cause of heart disease in western countries is atherosclerosis, an inflammatory process that affects the vessel wall of large and medium-sized arteries. Genetic and environmental factors associated to the disease include elevated circulating levels of low-density lipoprotein (LDL) and of very low-density lipoprotein (VLDL), obesity, diabetes, high blood pressure, a high-fat diet and smoking (Libby, 2002). The acute coronary syndrome, including myocardial infarction and stroke, results from the erosion or the rupture of the fibrous cap which surrounds the luminal side of the atherosclerotic lesion, leading to thrombus formation (Libby et al., 2006). Henney and coworkers first observed increased MMP3 expression in human atherosclerotic plaque where the enzyme might erode the connective tissue, leading to vessel wall fissuring and/or rupture of the plaque itself, and drive the aberrant activation of pro-MMP-9 and other MMPs. Cell-specific stromelysin expression in human atherosclerotic plaques was studied by in situ hybridization and immunocytochemistry (Henney et al., 1991). Stromelysin mRNA transcripts were localized in isolated individual cells, mainly smooth muscle cells, in the plaque cap, intima, and adventitia, but not in the media, and in foam cells (Okamoto et al., 2001). The isolated expression of stromelysin-1 by smooth muscle cells may reflect local connective tissue remodeling associated with plaque growth and formation, whereas the more extensive expression observed in macrophages may be of greater pathological significance, contributing to the destabilization of the extracellular matrix and eventually to plaque rupture (Henney et al., 1991).

On the other hand, different studies suggest an athero-protective role by MMP-3. An investigation on the potential role of stromelysin-1 (MMP-3) in the development and progression of atherosclerotic lesions and aneurysm formation has been undertaken, employing mice with a deficiency of apolipoprotein $\mathrm{E}(\mathrm{ApoE}(-/-)$ :MMP-3(+/+)) or with a combined deficiency 
of apoE and MMP-3 (ApoE(-/-):MMP-3(-/-)), which were fed with a cholesterol-rich diet (Silence et al., 2001). Atherosclerotic lesions throughout the thoracic aorta were significantly larger in $\mathrm{ApoE}(-\mid-)$ : $\mathrm{MMP}-3(-\mid-)$ than in $\mathrm{ApoE}(-\mid-)$ : MMP-3(+/+) mice and contained more fibrillar collagen. MMP-3 depletion was further associated to decreased incidence of abdominal aortas aneurysm. Histological analysis revealed enhanced accumulation of macrophages in atherosclerotic lesions of $\mathrm{ApoE}(-/-)$ :MMP-3(+/+) mice in association to an increased expression of urokinase-type plasminogen activator (u-PA) and of MMP-3. These findings suggest that plasmin activates the pro-MMP-3 pool released by macrophages and MMP-3 activity may then contribute to a reduction of plaque size by degrading matrix components, but at the same time it can affect artery wall integrity by cleaving elastin (Silence et al., 2001).

A common polymorphism in the promoter sequence (the $5 \mathrm{~A} / 6 \mathrm{~A}$ polymorphism) of the human stromelysin-1 gene associates the enzyme to an increased risk of coronary heart disease progression: in vitro experimental evidences indicated that these promoter variants can be recognized with different affinity by nuclear factors, leading to variable transcriptional efficiency of MMP-3 gene (Ye et al., 1996).

5.1.3.1.2. Aneurysm. MMP-3 overexpression has been detected in human abdominal aortic aneurysms (Knox et al., 1997; Thompson et al., 2002). In aneurysm a 40-fold increase in MMP-3 expression and a 200-fold increase in TIMP-3 concentration have been detected. The observation clearly suggests that upregulation of stromelysin-1 (MMP-3) and TIMP-3 may play a significant role in aneurysm genesis, but molecular mechanisms are far from being understood. It seems anyway plausible that an excessive production of stromelysin- 1 in the atherosclerotic aorta weakens the aortic wall and brings about additional proteolysis by activating other latent, constitutively expressed metalloproteinases (Choke et al., 2005). The origin of this increased stromelysin-1 expression in certain individuals prone to aneurysm formation is not known, but a combination of genetic and environmental factors may contribute (Carrell et al., 2002).

5.1.3.2. Diseases of the nervous system. Parkinson's disease (PD) is determined by a degeneration of the dopamine-synthesizing cells of the mesostriatal-mesocortical neuronal pathway (Vernier et al., 2004). Dopamin (DA) ergic neurons are particularly vulnerable being exposed to toxic by-products of DA catabolism.

Experimental findings associate MMP-3 to DAergic neurons degeneration: in MMP-3 deficient mice, the number of DAergic neurons in the substantia nigra was considerably higher than that of wild type mice (WT). Cultured mesencephalic neurons from MMP-3 KO showed higher [(3)H]DA uptake capability associated with a greater number of tyrosine hydroxylase-immunopositive neurons and an increased length of average dendritic branches (Kim et al., 2010).

MMP-3 is induced and activated in dopaminergic (DA) cells upon stress conditions. This has important consequences since MMP-3 cleaves $\alpha$-synuclein in vitro and in vivo and $\alpha$-synuclein and MMP-3 co-localize in Lewy bodies (LB), as observed in post-mortem brains of PD patients (Choi et al., 2011). Evidence from in vitro investigations suggests that the MMP-3 mediated C-terminal truncation of $\alpha$-synuclein releases fragments with the tendency to aggregate. These results strongly indicate that MMP-3 digestion of $\alpha$-synuclein in DA neurons could play a pivotal role in the progression of PD (Choi et al., in press).

\subsubsection{Diseases of joints and muscular system.}

5.1.3.3.1. Joint diseases. Excessive degradation of the cartilage ECM is a feature of two major joint diseases, osteoarthritis (OA) and rheumatoid arthritis (RA). In OA, the most common form of arthritis, an increased production of proteinases by chondrocytes leads to the breakdown of cartilage, while synovitis occasionally develops in early phases of the disease. On the other hand, RA, the most refractory and destructive form of arthritis, is characterized primarily by prominent and persistent synovitis and an expansion of inflammation which leads to cartilage and bone destruction. Articular cartilage breakdown by proteinases follows three mechanisms: (i) destruction from the surface of the articular cartilage by proteinases of the synovial fluid; (ii) destruction through direct contact of proteolytic synovium and/or pannus tissue with the articular cartilage; and (iii) intrinsic destruction by proteinases derived from chondrocytes (Okada, 2009).

In human OA cartilage MMP-3, together with other MMPs, is up-regulated by chondrocytes (Okada, 2009). The pathogenic activity relies on the activation of pro-MMP-1, pro-MMP-7, pro-MMP-8, pro-MMP-9 and pro-MMP-13 and digestion of ECM components, such as aggrecan and type IX collagen (Okada, 2009).

However, in an OA model of MMP-3 deficient mice, accelerated cartilage destruction occurred to a greater extent with respect to wild-type mice (Clements et al., 2003; Mudgett et al., 1998). The molecular mechanisms underlying MMP-3 pathogenic activity are not clear, since aggrecan cleavage could contribute to the generation of remnant epitopes (VDIPEN) (Mudgett et al., 1998; Sandy et al., 1991; Van Meurs et al., 1999).

Synovial fluid from RA patients shows aberrant concentration of MMP-3 and the MMP-3/TIMP ratio is also unbalanced (Okada, 2009; Yoshihara et al., 2000). MMP-3 is further detectable in serum samples from RA patients and is clinically accepted as a biomarker for RA diagnosis, for joint destruction prognosis and for RA treatment monitoring (Yoshihara et al., 2000).

5.1.3.4. Wound healing. Wound tissue in humans overexpresses multiple MMPs including MMP-3. This MMP may play a role in the complex process of wound healing that includes acute inflammatory reaction, regeneration of parenchyma cells, cell migration and proliferation, angiogenesis, contraction and tissue remodeling (Gill and Parks, 2008). As a matter of the fact MMP-3 knock out mice show impaired excisional reepithelization (Madlener et al., 1998). 
5.1.3.5. Diseases of the respiratory system. Acute respiratory distress syndrome (ARDS) is induced by an increase in the alveolar-capillary barrier permeability and the subsequent impairment of gas-exchange. The disease can progress to chronic fibrotic lung injury (Ware and Matthay, 2000). Since the alveolar basement membrane is primarily composed of type IV collagen, MMP-3 is likely involved in the extensive ECM remodeling observed in ARDS.

In order to determine the involvement of individual MMPs in the development of lung injury, MMP-3 and MMP-9 knockout mice and wild type mice were acutely injured with immunoglobulin $\mathrm{G}$ immune complexes and the intensity of the lung injury compared. In wild type mice upregulation of gelatinase B and stromelysin-1 was observed in the injured lungs. As a consequence, the MMP-3 and MMP-9 deficient mice had less severe lung injury, suggesting that both MMPs are involved in the lung injury pathogenesis. Nonetheless, the activity of MMP-3 and MMP-9 differs for some still obscure molecular aspects that lead to a reduced recruitment of neutrophils exclusively in the MMP-3 deficient mice (Warner et al., 2001a).

\section{2. $M M P-10$}

Stromelysin-2 or MMP-10 has been firstly identified in mouse and later in humans (Muller et al., 1988). The structure displays a $82 \%$ sequence homology with MMP-3 and it is secreted as a 53-kDa proenzyme (Sirum and Brinckerhoff, 1989). No structural details are available, even though it is postulated that it consists on a pro-domain, a catalytic domain and a hemopexin-like domain.

\subsubsection{Biological aspects}

MMP-10 digests a number of ECM molecules and participates in pro-MMPs activation, although the catalytic activity toward type IV and type V collagens is quite weak in comparison to that of the related MMP-3 (Murphy et al., 1992; Nagase et al., 2006). Inflammatory stimuli induce its release by synovial fibroblasts, whereas treatment with pro-catabolic elements stimulates expression by articular chondrocytes (Barksby et al., 2006). The physiopathological relevance of MMP-10 has been associated to vascular development, atherothrombosis (Montero et al., 2006; Rodriguez et al., 2008), skin wound healing and cell migration, since it is primarily found at the front of the migrating epithelial 'tongue' (Madlener and Werner, 1997) and it has been observed in migrating enterocytes in inflammatory bowel disease (Salmela et al., 2004; Vaalamo et al., 1998). MMP-10 is in vitro capable of processing laminin-5, which should be an additional mechanism through which the enzyme assists cell migration (Krampert et al., 2004). MMP-10 activity at sites of developing bones resorption has been demonstrated by histochemistry and by in situ casein zymography: this observation suggests a role in remodeling events occurring during ossification (Bord et al., 1998).

Elevated concentrations of the enzyme have been further detected in some human tumors in association to aggressiveness: a prognostic relevance for its quantification has also been proposed (Gill et al., 2004).

MMP-10 up-regulation in early phases of skin tumor growth is stimulated by TGF- $\beta$ and EGF: the resulting high collagenolytic activity correlates with invasiveness (Wilkins-Port et al., 2009). Increased levels of MMP-10 have been also found in lung cancer (Frederick et al., 2008).

\section{3. $M M P-11$}

\subsubsection{Structural peculiarities and biological aspects}

Stromelysin-3 (MMP-11) together with matrilysin (MMP-7) and metalloelastase (MMP-12) is often included in the stromelysin subgroup, although they are structurally less closely related to MMP-3 and MMP-10. MMP-11 was first identified in 1990 in a cDNA library extracted from human breast cancer cells and, though sharing with other MMPs the classic domain composition (see Fig. 1), it deserves some additional comment (Basset et al., 1990). Thus, the 56-kDa pro-MMP11 is activated in the intracellular compartment by furin and Golgi associated furin-like proteases (Pei and Weiss, 1995) or by paired basic amino acid cleaving enzyme-4 (PACE-4) (Bassi et al., 2000); it is then secreted as a 47-kDa active protease. This intracellular activation was shown to be dependent on a unique furin recognition motif RNRQRKR located at the junction between the pro- and the catalytic domain (Pei and Weiss, 1995). Furthermore, the relatively narrow and peculiar substrate specificity observed for MMP-11 probably stems from a mutation that occurs in the highly conserved methionine turn; thus, where all other MMPs contain an MxP sequence in the Met turn, in MMP-11 the proline is replaced by an alanine. This substitution has a profound effect on the structure of the S1' selectivity pocket leading to greatly changed substrate specificity (Noel et al., 1995).

The 3D-structure of the catalytic domain of mouse MMP-11 in a complex with a phosphinic peptide inhibitor has been solved (Gall et al., 2001). The S1' cavity appears as a long channel able to accommodate side chains longer than those present in natural amino acids. This observation fits perfectly with the ability of MMP-11 to cleave with high efficiency substrates containing in their P1' position unusual amino acid with very long side chains (Gall et al., 2001).

Beside the pro- and mature forms of human MMP-11, several other forms of MMP-11 have been also reported. In an in vitro stromal/epithelial cell co-culture model, a 35-kDa protein lacking enzymatic activity was found to be produced by normal pulmonary fibroblasts (Mari et al., 1998). Two forms of 35 and $28 \mathrm{kDa}$ were also observed in human atherosclerotic tissues (Schönbeck et al., 1999).

Matrix metalloproteinase-11 is widely expressed in both normal and pathological remodeling processes, such as embryonic implantation (Lefebvre et al., 1995), placentation, interdigitation, osteogenesis, spinal cord morphogenesis, epithelium 
growth, larval tissue resorption (Berry et al., 1998), morphogenesis, menstrual breakdown, post-partum involution, ovulation (Hägglund et al., 1999), post-weaning involution, inflammation, wound healing (Wolf et al., 1992), benign tumor proliferation, and tissue invasion (Rio, 2005; Rouyer et al., 1994; Thewes et al., 1999). In both normal and pathological conditions, MMP-11 is mostly secreted by fibroblasts.

MMP-11 expression is maximal in uterus, placenta, and involuting mammary gland (Visse and Nagase, 2003) and is negatively modulated by progesterone treatment, at least in isolated stromal cells in vitro (Osteen et al., 2003). Timing of MMP11 expression in tissue remodeling is well defined; thus, it is induced at an advanced stage, suggesting that it does not take part into the basement membrane breakdown at early phases, but rather in downstream events when the connective compartment remodeling is suitable for epithelial cells incoming and tissue regeneration. Intriguingly, MMP-11 exhibits an antiapoptotic function, an unusual feature for a MMP.

Despite the identification of possible in vitro substrates for MMP-11 (Matziari et al., 2007), there is lack of convincing evidences that support their in vivo cleavage (see Table 3). Identification of the in vivo physiological substrates of MMP-11, as well as the development of potent and specific inhibitors for this particular MMP family member would certainly provide valuable information about the physio-pathological roles of MMP-11.

However, several putative substrates mainly include protease inhibitors, such as $\alpha_{1}$ proteinase inhibitor and $\alpha_{2}$ macroglobulin (see Table 3). Further, MMP-11 has a weak caseinolytic activity and it has been shown to cleave insulin-like growth factor-binding protein-1 (IGF-BP-1) in a carcinoma cell line (Pei et al., 1994). Recent research has demonstrated that, although MMP-11 does not cleave many ECM proteins, degradation of type VI collagen could be physiologically related to adipogenesis inhibition (Motrescu et al., 2008).

\subsubsection{Neoplastic diseases}

It has been shown that MMP-11, among other MMPs, interacts with stromal components that contribute to malignancy in the early and late stages of human tumors progression (Basset et al., 1997; Coussens and Werb, 1996).

Clinical investigations show that an overexpression of MMP-11 is correlated to a lower survival rate in patients with breast, head, neck or colon cancer (Noel et al., 2000; Muller et al., 1993; Porte et al., 1995). Interestingly, as observed for MMP-9, MMP-11 is not exclusively released by malignant cells, but also by the surrounding mesenchymal cells (Thewes et al., 1999).

In carcinomas, both stromelysin-3 RNA and protein have been specifically detected in fibroblasts surrounding cancer cells. In agreement with this observation, carcinomas with the tendency to progress without inducing a prominent stroma are also those which usually do not express the ST3 gene. ST3 gene expression was also observed in non-invasive carcinomas of the breast, uterus cervix and bladder, where the probability of detecting ST3 RNA and protein positively correlated with invasiveness. Taken all together, these observations further support the hypothesis that ST3 may contribute to tissue-remodeling processes associated with carcinoma progression (Rouyer et al., 1994).

In addition, even though MMP-11 is essential for tumor development, it does not share any function with the other MMPs involved in malignant events. MMP-11 is not able to degrade any major ECM component, it does not modify epithelial cell proliferation or motility, and it does not appear to be a pro-angiogenic or a pro-apoptotic factor (Mari et al., 1998). MMP-11 may either control proteinase activity, survival factor bioavailability, or inflammatory reactions to favor cell survival in an environment initially not adequate for epithelial cell growth. Nonetheless, in MMP-11-deficient mice tumors exhibit higher levels of apoptosis, and implantation of experimental tumors is lower in MMP-11 null mice (Boulay et al., 2001). Therefore, it may be hypothesized that, in addition to proteinases, proteinase inhibitors, and insulin-like growth factor binding proteins (IGF-BPs), MMP-11 may hydrolyze yet uncharacterized specific substrates, and therefore, it could exerts the pathogenic activity through different mechanisms (Rio, 2002).

\section{Matrilysins}

Matrylisin family includes two enzymes: MMP-7 (also known as Pump-1) and MMP-26 (De Coignac et al., 2000; Quantin et al., 1989). These enzymes display significant sequence similarities with collagenases and stromelysins, even though the domain composition lacks the hemopexin-like domain (see Fig. 1) (Uria and Lopez-Otin, 2000).

A vast deal of knowledge defines a relevant physiopathological role for MMP-7, whereas biological aspects of MMP-26 should be restricted to extracellular matrix turn-over and remodeling in a limited cohort of tissues both in physiological and pathological conditions (Park et al., 2000; Marchenko et al., 2001).

MMP-7 is widely expressed in human tissues and mainly in the epithelial-derived ones. The catalytic activity drives proteolytical breakdown of several extracellular matrix molecules (see Table 3), such as cytokines, growth factors and receptors (Li et al., 2002). MMP-7 biological functions mainly concerns ECM remodeling and immune system modulation. Therefore a un-balanced activity of the enzyme is associated to many human pathological conditions, mostly including cancer and cardiovascular, respiratory and neuronal diseases. In particular, MMP-7 affects almost all phases of tumorigenesis through molecular mechanisms that, at least partially, overlap with that of other soluble MMPs, as widely discussed in the present review (Adachi et al., 1999; Li et al., 2002; Tan et al., 2005). This evidence suggests the existence of not yet identified interactions with other factors that contribute in defining a complex MMP-7 biology. 


\subsection{MMP-7}

\subsubsection{Biological aspects}

MMP-7 (generally called as matrilysin) was originally described as Pump-1 (putative uterine metalloprotease-1) in 1988 (Muller et al., 1988). The MMP-7 cDNA codes for a protein with significant sequence similarity to collagenases and stromelysins; however, it lacks the hemopexin-like domain (see Fig. 1). Pump-1 expressing COS cells secrete a 28 kDa pro-protein; activation by organomercurials agents, leads to two active Pump-1 species of 21 and $19 \mathrm{kDa}$ (Imai et al., 1995).

MMP-7 has been shown to be constitutively expressed in the ductal and glandular epithelium of normal mammary, peribronchial and parotid glands: it is further represented in liver, pancreas, prostate, and airways (Harrell et al., 2005). As other soluble MMPs, activity of MMP-7 is tightly regulated: in particular GAGs have been reported to be key modulators of MMP-7 autolytic activation, but several other not identified macromolecules should contribute (Ra et al., 2009).

Active MMP-7 cleaves ECM and basement membrane proteins, such as fibronectin, collagen IV, laminin, and particularly elastin, entactin, and cartilage proteoglycan aggregates (see Table 3); in addition, it degrades casein, gelatin, collagen of I, III and V, and can activate collagenases (Quantin et al., 1989). Furthermore, MMP-7 appears to mediate the proteolytic processing of other molecules, playing an important role in ectodomain shedding of cell-surface molecules, such as tumor necrosis factor- $\alpha$ (TNF- $\alpha$ ) precursor, Fas ligand (FasL), heparin binding epidermal growth factor (HB-EGF), E-cadherin, and $\beta 4$-integrin (Ii et al., 2006; Wilson and Matrisian, 1996).

Distribution of MMP-7 in the glandular epithelium is of particular biological relevance. The release in either the apical and/or the basolateral side is linked to different processes: apical MMP-7 activity is associated to proliferation induction (Harrell et al., 2005).

MMP-7 has also an important role in the homeostasis of innate immunity in lungs and intestine, where it proteolytically activates anti-bacterial peptides (e.g., pro-defensins) and mediates release of TNF- $\alpha$ by macrophages (Burke, 2004). Consistently with this role in innate immunity, MMP-7 is induced by microbial products and also by hypoxia (Burke, 2004; Wilson et al., 1999).

The influx of inflammatory cells to sites of injury is largely directed by signals from the epithelium, but how these cells form chemotactic gradients is not known. In MMP-7-null mice, neutrophils remained confined in the interstitium of injured lungs and do not advance into the alveolar space. Impaired transepithelial migration is accompanied by a lack of both shed syndecan-1, a heparan sulfate proteoglycan, and KC, a CXC chemokine, in the alveolar fluid. In vitro, MMP-7 cleaves syndecan-1 from the surface of cells; therefore, the matrilysin-mediated shedding of syndecan-1/KC complexes from the mucosal surface addresses neutrophil influx toward sites of injury (Li et al., 2002). Furthermore, since MMP-7 mediates shedding of E-cadherin ectodomain from injured lung epithelium both in vitro and in vivo, transfection of activated matrilysin in alveolar-like epithelial cells results in shedding of E-cadherin and accelerated cell migration (McGuire et al. 2003).

\subsubsection{Neoplastic diseases}

6.1.2.1. Epidemiological aspects. The role of MMP-7 in cancer has been well described. The MMP-7 source substantially differs from that of other MMPs, since it is almost exclusively released by cancerous cells (Ii et al., 2006). As with other MMPs, MMP7 has been identified in a wide range of tumors, and correlates with tumor aggressiveness.

MMP-7 is overexpressed in a variety of epithelial tumors, mesenchymal tumors and in invasive cancers of the digestive tract. Matrilysin expression in carcinoma cells at the invasive front was immuno-histochemically detected in patients with esophageal squamous cell carcinoma (SCC) and was associated to an advanced tumor stage (Yamamoto et al., 1999).

Matrilysin promotes in vitro invasiveness of cancer cells of esophagus, stomach, colon, liver and pancreas (Adachi et al., 1999, 2001; Jones et al., 2004; Tan et al., 2005; Yamamoto et al., 1999; Yamamoto et al., 2001).

MMP-7 is particularly overexpressed in early undifferentiated gastric carcinoma where it is considered a predictive factors for invasiveness and metastatization: hence, MMP-7 quantification could be of prognostic relevance (Adachi et al., 2001, 1999; Aihara et al., 2005).

MMP-7 shows a peculiar distribution in human pancreatic adenocarcinomas; its expression is usually compartmentalized at the leading edge of invading cells (Bolon et al., 1997).

In all these cases, a matrilysin-positive carcinoma predicts a significantly shorter survival, since it correlates with primary tumor growth, regional lymph nodes colonization and distant metastasis (Jones et al., 2004; Yamamoto et al., 2001).

Moreover, an interesting observation is that the functional polymorphism in the matrilysin promoter $(-181 \mathrm{~A} / \mathrm{G})$ has been shown to increase susceptibility to esophageal squamous cell carcinoma, gastric cardiac adenocarcinoma, and non-small-cell lung carcinoma (Zhang et al., 2005a).

6.1.2.2. Tumorigenesis. Modulation of soluble molecules availability is a major mechanism through which MMP-7 promotes early tumorigenesis. MMP-7 sheds the ectodomain of HB-EGF precursor (pro-HB-EGF) to yield mature HB-EGF, which, upon bindings to the ErbB4 receptor promotes cellular proliferation and inhibits apoptosis (Yu et al., 2002).

Furthermore, recent epidemiologic studies have focused on a correlation between high levels of circulating insulin-like growth factor (IGF-1) and low levels of IGF binding protein 3 (IGFBP-3) and the consequent risk of developing colon, breast, prostate, and lung cancer. MMP-7 cleaves IGFBP-3, hence playing a crucial role in modulating IGF-I bioavailability. This mechanism may promote tumorigenesis of MMP-7-producing IGF-I-receptor-expressing tumors in both primary and 
secondary sites (Miyamoto et al., 2004). ADAM28, a member of the disintegrin and metalloproteinase (ADAM) family (see Section 1.1.2), is also a substrate of MMP-7 and it has been reported that the secreted form of ADAM28, once activated by MMP-7, also digests IGFBP-3 even when complexed with IGF-I or IGF-II (Mochizuki et al., 2004).

MMP-7 further contributes to invasiveness by cleaving ECM substrates and by increasing the activation rate of pro-MMP2 and pro-MMP-9 (Wang et al., 2005, 2006). The function of adhesiveness-related molecules can be altered by cleavage and subsequent release of their ectodomains. The E-cadherin ectodomain is constitutively shed from the surface of MCF-7 and MDCK cells in culture. Matrilysin cleaves E-cadherin (E-CAD) releasing a $80 \mathrm{kDa}$ soluble E-cadherin fragment (sE-CAD) into the medium. The soluble E-cadherin fragment then inhibits E-cadherin functions in a paracrine way, impairing adhesiveness and promoting tumor cells migration and invasion (Noe et al., 2001).

Another interesting MMP-7-linked pathological phenomenon in advanced prostate tumors is the cancer-induced bone resorption: MMP-7 is produced by osteoclasts at the tumor-bone interface where it cleaves the receptor activator of nuclear factor kappa-B ligand (RANKL) whose soluble form promotes osteoclast activation. MMP-7-deficient mice demonstrated reduced prostate tumor-induced osteolysis and RANKL processing (Lynch et al., 2005).

A relevant mechanism through which MMP-7 affects tumor growth is the modulation of apoptosis. MMP-7 processes the recombinant and the cell-associated Fas ligand (FasL) to soluble FasL which induces apoptosis in epithelial cells (O'Connell et al., 1999; Powell et al., 1999). However, there are contradictory evidences about the association of MMP-7 and Fasmediated apoptosis which reflect the presence of different cleavage sites which are linked to variable biological effects (Hohlbaum et al., 2000; Schneider et al., 1998; Tanaka et al., 1998; Vargo-Gogola et al., 2002).

6.1.2.3. Angiogenesis. Angiogenesis is a process essential for tumor progression. MMP-7 mRNA and protein are found in vascular endothelial cells in close apposition to matrilysin-positive tumor cells (Nagashima et al., 1997). Furthermore, MMP-7 may accelerate the proliferation of human umbilical vein endothelial cells in a dose-dependent manner in vitro (Huo et al., 2002). Moreover, in a murine experimental model, matrilysin-induced angiogenesis was inhibited by the administration of a matrilysin-specific antisense oligonucleotide (Nishizuka et al., 2001).

On the other hand, there is evidence that MMP-7 activity further produces anti-angiogenic factors. In this respect, MMP-7 cleaves human plasminogen releasing an angiostatin-like fragment (Patterson and Sang, 1997). In addition, endostatin, an angiogenesis inhibitor, and neostatin-7, the C-terminal 28-kDa endostatin-spanning proteolytic fragment (Chang et al., 2005), are generated from collagen type XVIII by matrilysin in vitro (Heljasvaara et al., 2005) and in vivo (Lin et al., 2001). Matrilysin further cleaves a matrix-bound isoform of vascular endothelial growth factor (VEGF), releasing it from its natural reservoir (Lee et al., 2005).

\subsubsection{Non-neoplastic diseases}

6.1.3.1. Diseases of the cardiovascular system. MMP-7 deletion has been shown to improve survival after myocardial infarction (MI) (Chiao et al., 2010). In particular, MMP-7 is involved in N-cadherin cleavage and in vascular smooth muscle cell (VSMC) apoptosis, leading to fibrous cap thickness and to plaque instability (Williams et al., 2010). Nonetheless, MMP-7 is increased in patients with stable and unstable coronary artery disease (Nilsson et al., 2006).

Furthermore, MMP-7 modulates the transcription of ADAM12, the main protease implicated in cardiac hypertension and hypertrophy (Wang et al., 2009) (see Section 1.1.2): hence, knockdown of MMP-7 results in an attenuation of these pathological conditions.

MMP-7 involvement in hypertension is further mediated by signaling of adrenoreceptors and angiotensin receptors that lead to MMP-7 activation, which, in turn, sheds EGFR ligands (such as HB-EGF), thereby promoting EGFR-dependent vasoconstriction (Hao et al., 2004).

MMP-7 is also associated to histopathological alterations in human aneurysms and dissections of the thoracic ascending aorta; immunohistochemical investigations reveal MMP-7 over-expression in areas of mucoid degeneration within the medial layer. This pathological process correlates with areas of cell disappearance and disruption of extracellular matrix elastic and collagen fibers (Borges et al., 2009).

6.1.3.2. Diseases of the nervous system. In multiple sclerosis (MS) where transmigration of pathogenic T cells across the bloodbrain barrier (BBB) is facilitated by the expression of cell adhesion molecules and proteinases that degrade the ECM (Pachter et al., 2003), MMP-7 contributes by cleaving proteins that are essential for blood-brain barrier integrity and immune suppression. Thus, elevated levels of MMP-7 have been reported in human MS patients and in a delayed-type hypersensitivity model for MS (Anthony et al., 1998). MMP-7 may facilitate immune cell access or re-stimulation in perivascular areas, which are critical events in experimental autoimmune encephalomyelitis and multiple sclerosis, and provide a new therapeutic target to treat this disorder (Buhler et al., 2009).

Pathological evidence suggests that alterations of the blood-brain barrier (BBB) may occur in association with human immunodeficiency virus (HIV) dementia (HIVD). Also in this pathological process circulating levels of MMP-7 have been found to correlate with the severity of brain injury in infected subjects (Ragin et al., 2009). In this case, the cerebrospinal fluid (CSF) of patients with HIVD shows increased levels of pro-MMP-7, suggesting that elevated CSF levels of MMP-7 may reflect immune activation within the central nervous system (Conant et al., 1999). In general terms, CSF levels of MMP-7 are often significantly correlated with neural injury in multiple brain regions (Ragin et al., 2011). 
Interestingly, it has been reported that MMP-7 activity is linked to disruption of mature synapses in hippocampus: therefore, MMP-7 could be an effector of neuronal loss in each pathological conditions which determine its up-regulation (Bilousova et al., 2006).

6.1.3.3. Diseases of joints and muscular system. Pro-MMP-7 is overexpressed in osteoarthritic (OA) articular cartilage (Okada, 2009). A recent study on human OA cartilage shows that pro-MMP-7 is activated through the interaction with tetraspanin CD151, leading to cartilage destruction and/or chondrocyte cloning (Fujita et al., 2006).

Dupuytren's disease (DD) is a common fibrotic condition of the palmar fascia, leading to the deposition of collagen-rich cords and finger contractions. The expression of MMP-7 is increased in the DD nodule and remains equally expressed in the DD cord (Johnston et al., 2007).

In inflammatory myopathies a MMP-7 over-expression has been demonstrated in areas with high levels of expression for the major-histocompatibility-complex class I (MHC-I) in atrophic and invaded myofibers as well as in CD-8 positive lymphocytes and macrophages (Schoser et al., 2002).

6.1.3.4. Diseases of the respiratory system. Idiopathic pulmonary fibrosis (IPF) is a chronic, usually fatal, disorder characterized by excessive matrix degradation and interstitial and intraalveolar fibrosis leading to dyspnea, impaired oxygen transfer and alveolar collapse (Katzenstein and Myers, 1998). MMP-7 is identified as a potential target for therapy of patients with IPF, since MMP-7 gene is up-regulated, as from micro-array gene analyses (Cosgrove et al., 2002). Furthermore, matrilysin knockout mice were dramatically protected from pulmonary fibrosis in response to intratracheal bleomycin (Zuo et al., 2002).

MMP-7 may also be a modulator of pediatric lung disease; thus, MMP-7 levels are elevated in endotracheal tube aspirates from patients with different respiratory syndromes (ETAs) (Winkler et al., 2003).

A putative involvement of MMP-7 in tuberculosis has been demonstrated as well. Thus, MMP-7 (and MMP-1, see Section 3.1.3.2) gene expression and secretion are potently upregulated by M. tuberculosis, and no increase in tissue inhibitor of metalloproteinase expression occurs to oppose their activity. These two MMP can then degrade the major lung ECM components, such as type I collagen and elastin (Elkington et al., 2005).

\section{2. $M M P-26$}

\subsubsection{Structure}

MMP-26 (matrilysin-2, endometase) has been identified in an endometrial tumor (De Coignac et al., 2000). The isolated cDNA encodes a 261 amino acids polypeptide, the smallest MMP identified to date, which contains several structural features of MMPs including the signal sequence, the prodomain involved in enzyme latency, and the catalytic domain with the zinc-binding site (see Fig. 1). However, it lacks the hinge region and the hemopexin-like domain (Uria and Lopez-Otin, 2000). The amino acid sequence of matrilysin-2 also contains a threonine residue adjacent to the $\mathrm{Zn}$-binding site that has been defined as a specific feature of MMP-26 (Uria and Lopez-Otin, 2000). MMP-26 is unique among MMPs, since it is the only known MMP (up to now), which does not have a functional cysteine switch mechanism to maintain the pro-enzyme in its latent conformation. Loss of the cysteine switch is attributed to the presence of a histidine residue N-terminal to the cysteine, a feature that is exclusively observed in MMP-26 (Marchenko and Strongin, 2001; Marchenko et al., 2001).

\subsubsection{Biological aspects}

MMP-26 hydrolyzes type IV collagen, fibronectin, fibrinogen, and gelatin, but it cannot degrade laminin and elastin (Park et al., 2000); in addition, MMP-26 is able to activate progelatinase B (Uria and Lopez-Otin, 2000). Expression analyses revealed that matrilysin- 2 is detected in placenta, in endometrium and uterus, but is widely expressed in a wide range of malignant tumors in vivo and in vitro (Marchenko et al., 2001; Uria and Lopez-Otin, 2000). These data together with the broad spectrum of proteolytic activity, suggest that matrilysin-2 may play a role in tissue-remodeling events associated to tumor progression.

\section{Membrane type-MMPs}

The first human MT-MMP (MT1-MMP/MMP-14) (Sato et al., 1994) was cloned by a reverse transcriptase-polymerase chain reaction (RT-PCR) from the RNA of placental tissue. Later on, additional studies, focused on MT-MMPs, led to the discovery of five more members of this family, namely MT2-MMP (MMP-15), MT3-MMP (MMP-16), MT5-MMP (MMP-24) and two additional members, MT4-MMP (MMP-17) and MT6-MMP (MMP-25), which are anchored to the plasma membrane via a glycosylphosphatidylinositol (GPI) domain (Zucker et al., 2003).

\subsection{Structure and activation of MT-MMPs}

The MT-MMPs contain all protein domains characteristic of MMPs from the N-terminal to the C-terminal extremities, that is a signal peptide, a propeptide, a catalytic domain with the zinc binding site, a hinge region and a C-terminal hemopexin 
domain (Sternlicht and Werb, 2001). However, MT-MMPs differ from the other MMPs by the presence of a C-terminal domain rich in hydrophobic residues and involved in their association with the cell membrane (see Fig. 1).

According to the structure of this C-terminal extension, MT-MMPs can be classified into two sub-groups (see Fig. 1 and Table 2): (a) type I transmembrane proteins including MT1-, MT2-, MT3- and MT5-MMP characterized by a long hydrophobic sequence followed by a short cytoplasmic tail and (b) glycosylphosphatidylinositol (GPI)-type MT-MMPs (MT4- and MT6-MMP) containing a short hydrophobic signal anchoring to GPI. This sequence is not followed by a cytoplasmic tail. The cytoplasmic tail of type I transmembrane MT-MMPs is likely participating in numerous cellular events, such as cell signaling, sub-cellular localization, MT-MMP trafficking and dimerization (Gingras et al., 2001; Itoh et al., 2001; Rozanov et al., 2001; Sounni et al., 2004; Uekita et al., 2004; Wang et al., 2004a).

The prodomain of MT-MMPs contains a conserved motif, Tyr42- Gly43-Tyr44-Leu45 that acts as an intramolecular chaperone. It appears essential for adequate protease folding and enzymatic activities (e.g., proMMP-2 activation, substrate degradation and TIMP-2 binding) (Pavlaki et al., 2002). A basic tetrapeptidic sequence Arg108-Arg109-Lys110-Arg111 is inserted between the propeptide and the catalytic domain (see Fig. 1). This furin recognition motif is cleaved by proprotein convertases in the trans Golgi network during the trafficking of MT-MMPs from the endoplasmic reticulum to the plasma membrane (Hotary et al., 2000; Pei and Weiss, 1996; Rozanov et al., 2001; Yana and Weiss, 2000; Zucker et al., 2003). However, the activation of MT-MMPs by a furin-independent alternative pathway has been reported only in some types of cells. This alternative activation pathway depends on an autoproteolytic activation or on the action of non-furin proprotein convertases, or else on other proteases located at the plasma membrane (Cao et al., 1996; Rozanov et al., 2001; Sato et al., 1996; Yana and Seiki, 2002). In addition, an extracellular activation of proMT1-MMP by plasmin has been reported (Okumura et al., 1997). The propeptide is followed by the catalytic domain that contains the consensus zinc-binding motif HEBXHXBGBXH, where $\mathrm{X}$ is a variable residue and $\mathrm{B}$ is a bulky hydrophobic amino acid. The catalytic domain of MT-MMPs contains a characteristic 8 -amino acid insertion between stands bII and bIII named MT-loop. The 3D structure of the complex between the catalytic domain of MT1-MMP and TIMP-2 shows that the MT-loop generates a pocket in the MMP catalytic domain fold that interacts with the AB-loop of TIMP-2 (Fernandez-Catalan et al., 1998). The 3D structure of the complex between the MT3-MMP catalytic domain and a synthetic MMP inhibitor (namely batimastat) reveals similar features, but it shows unique properties, such as a modified MT-specific loop and a closed S1' pocket, which may be important for the substrate specificity and the efficient proMMP-2 activation (Lang et al., 2004). Although mutations or deletion of MT-loop of MT1-MMP do not affect its catalytic activity towards synthetic substrates, it impairs the enzymatic activation of proMMP-2 (English et al., 2001). Consistently, differences in the MT-loop structure between MT-MMPs are translated in their ability to activate proMMP-2. For example, MT4-MMP and MT6-MMP, which are lacking the MT-loop, are either unable or inefficient in activating proMMP-2 (English et al., 2001). Furthermore, human MT2-MMP is somewhat defective in cell-mediated activation of proMMP-2, whereas mouse MT2-MMP is very efficient in this activity. The replacement of two residues (Pro183 and Glu185) in the MT-loop of the human enzyme by the corresponding ones (Ser183 and Asp185) of mouse MT2MMP endowed the human enzyme with the capacity of an efficient activation of proMMP-2 (Miyamori et al., 2000).

Finally, MT-MMPs contain a C-terminal hemopexin-like domain that confers some degree of substrate specificity (Cataldo et al., 2003; Egeblad and Werb, 2002; Sounni et al., 2003).

\subsection{MT1-MMP (MMP-14)}

MMP-14, also referred to as MT1-MMP, which is the prototype membrane type MT-MMP, has been studied in the framework of renal development. This enzyme has intrinsic proteolytic capabilities and can also induce its effects by activating MMP-2 and MMP-13 (Itoh and Seiki, 2006).

\subsubsection{Biological features}

Although several data defined a role for MT1-MMP in renal development and suggested that its role was the cleavage ECM components in renal basal membranes (BM)s, the mechanisms by which the renal abnormalities occur is unclear. Pericellular cleavage of multiple BM components by MT1-MMP is also important for cell proliferation and migration and plays a critical role in normal kidney development (Riggins et al., 2010).

Numerous ECM components, including collagens I, II and III, fibronectin, vitronectin, laminins 111 and 332, fibrin and proteoglycans are substrates for MT1-MMP (Seiki, 2003). In addition, MT1-MMP can cleave other cell surface proteins, such as CD44 (Kajita et al., 2001), transglutaminase (Belkin et al., 2001), low-density lipoprotein receptor related protein (Rozanov et al., 2004), the integrin $\alpha \mathrm{v}$ subunit (Baciu et al., 2003; Deryugina et al., 2002a), and syndecan-1 (Endo et al., 2003). These highly divergent substrates (see Table 3) for MT1-MMP make this enzyme a critical regulator of the pericellular environment and allow it to regulate multiple cellular functions. The physiological importance of MT1-MMP was demonstrated by the multiple abnormalities observed in the MT1-MMP null mice, which die shortly after birth with severe musculoskeletal abnormalities characterized by decreased chondrocyte proliferation and decreased collagenolytic activity (Holmbeck et al., 1999; Zhou et al., 2000). More recent investigations on the musculoskeletal system have shown that reconstitution of MT1-MMP activity in the type II collagen expressing cells of the skeleton in MT1-MMP null mice rescues the diminished chondrocyte proliferation in these mice and ameliorates the severe skeletal dysplasia by enhancing bone formation (Szabova et al., 2009). In addition, these null mice have submandibular gland branching morphogenesis abnormalities (Oblander et al., 2005) as well as defects in lung development (Atkinson et al., 2005; Oblander et al., 2005), angiogenesis (Zhou et al., 2000) 
and myeloid cell fusion (Guadamillas et al., 2010). These deficiencies are ascribed to a lack of MT1-MMP catalytic ability, alterations in downstream pro-MMP-2 activation and alterations in cell functions regulated by the MT1-MMP cytoplasmic tail.

\subsubsection{Neoplastic diseases}

7.2.2.1. Epidemiologic aspects. Numerous studies have reported the expression of MT-MMPs, soluble MMPs activated through a MT-MMP-dependent process and their tissue inhibitors in various tumor types (Polette and Birembaut, 1998; Sato and Seiki, 1996; Seiki, 1999). MT1-MMP is mainly believed to be important for tumor invasion and metastasis. Its over-expression strongly promotes cellular invasion in vitro (Gilles et al., 2001; Hotary et al., 2000; Kajita et al., 2001; Sato et al., 1994; Seiki, 2003; Sounni et al., 2002a,b) and experimental metastasis (Habelhah et al., 1999; Shimada et al., 2000; Tsunezuka et al., 1996).

Clinical studies revealed that the expression of MT1-MMP and MMP-2 is associated with poor prognosis in patients with advanced neuroblastoma (Sakakibara et al., 1999), small cell lung cancer (SCLC) (Michael et al., 1999), tongue squamous cell carcinoma (Yoshizaki et al., 2001), head and neck carcinoma (Yoshizaki et al., 1997), bladder cancer (Kanayama et al., 1998), and ovarian cancer (Davidson et al., 2002). Low expression levels of MT1-MMP are considered as favorable survival markers in advanced colorectal carcinoma (Bendardaf et al., 2003). MT1-MMP has been detected in tumor cells and adjacent stromal cells in a variety of human tumors, including breast, cervical, colon, bladder, gastric, glioma, pancreatic, liver, ovarian, prostate and thyroid cancer (Nuttall et al., 2003; Zucker et al., 2003). Studies based on in situ hybridization analysis in lung (Polette et al., 1996) and breast carcinomas (Bisson et al., 2003; Okada et al., 1995) revealed that MT1-MMP mRNA is expressed by stromal cells surrounding tumor cells. Other studies have shown the expression of MT1-MMP in cancer cells rather than stromal cells of human thyroid, brain, head and neck cancer (Zucker et al., 2003).

There is clear evidence that MT1-MMP and MMP-2 are involved at different stages of tumor progression from the initial tumor development, growth and angiogenesis to invasion, metastasis and growth at secondary sites (Seiki et al., 2003; Sounni et al., 2003; Zucker et al., 2003). Many of these insights came from experiments with genetically modified animals or cells (see Table 6). For instances, MMP-14 over-expression in the mammary gland leads to the formation of a mammary carcinoma (Ha et al., 2001). Other studies in experimental cancer models demonstrated that human breast, melanoma and glioma cells transfected with MT1-MMP enhanced MMP-2 activation, invasion and in vivo tumor growth (Baramova et al., 1997; Deryugina et al., 2002b; Hotary et al., 2003; Iida et al., 2004; Itoh et al., 1998; Sounni et al., 2002a). It should be pointed out that MT1-MMP over-expression in human breast cancer can induce tumor growth, whereas the over-expression of MMP2 alone in the absence of MT1-MMP does not promote tumor development in nude mice (Sounni et al., 2002b; Tester et al., 2004). These observations suggest that MT1-MMP may contribute to the neoplastic progression independently of its capacity to activate pro-MMP-2. MT1-MMP is viewed as a tumor-derived growth factor that regulates proliferation by controlling cell geometry within the three-dimensional extracellular matrix. In fact, MMP-14 pericellular proteolysis confers a three-dimensional collagen matrix specific growth advantage. When MT1-MMP transfected cells are grown in gels composed of a mutant collagen that cannot be degraded, proliferation is blocked (Hotary et al., 2003).

MT1-MMP expression profile is likely to vary from one tumor type to another. Gliomas, the most common primary CNS malignancy in adults, is associated with upregulation of several MMPs (Kessenbrock et al., 2010). MT-MMPs appear to play a role of particular relevance for pericellular proteolysis, since MT1-MMP, MT2-MMP, MT5-MMP, and MT6-MMP are upregulated in glioma (Llano et al., 1999; Nuttall et al., 2003; Velasco et al., 2000). MT1-MMP plays a key role in glioma cell ability to spread and migrate on myelin (Paganetti et al., 1988; Amberger et al., 1994; Beliën et al., 1999). TIMP-2 could stimulate invasion through its interaction with MT1-MMP by promoting ERK signaling (Sounni et al., 2010).

Superficial spreading melanoma (SSM) and acral lentiginous melanoma (ALM) showed a moderate expression of MT1MMP. In metastatic melanoma, MT1-MMP is more intensely expressed (Ohnishi et al., 2001).

7.2.2.2. Tumorigenesis. The role of MT-MMPs as well as other MMPs in cancer metastasis and invasion could not be restricted to the ECM component degradation. Cell invasion is a multistep process involving ECM degradation and cell mobility. While ECM degradation can be orchestrated collectively by MMPs, cell migration is likely predominantly associated with MT1-MMP activity.

The ECM degradation is required for a cancer cell to degrade physical barriers during local expansion and intravasation at nearby blood vessels, extravasation and invasion at a distant location. During invasion, the localization of MMP proteolytic activity on specialized cell surface structures, called invadopodia, is a requisite for cancer cells ability to promote invasion. Invadopodia utilize transmembrane invadopodia-related proteinases, including MMP-14, for cancer cell proliferation: MMPs control proliferation signals through integrins because the shedding of E-cadherin results in $\beta$-catenin translocation to the nucleus, leading to cell proliferation. It is worth noting that the inactive pro-form of TGF- $\beta$, an important biomolecule in cancer, is proteolytically activated by MMP-14 (Mu et al., 2002).

Through its activity towards cell surface adhesion molecules (CD44, tTG and av integrin) (Belkin et al., 2001; Kajita et al., 2001; Ratnikov et al., 2002) or specific ECM protein such as laminin-5 (Gilles et al., 2001; Koshikawa et al., 2000), MT1-MMP can strongly modulate cell adhesion and cytoskeletal organization leading to cell mobility. The importance of endogenous MT1-MMP in cancer invasion was evidenced by downregulation of MT1-MMP in aggressive cancer cells using RNA silencing technology (Ueda et al., 2003). The specific silencing of MT1-MMP is sufficient to inhibit the invasion of highly invasive fibrosarcoma H1080 cells known to produce high levels of different MMPs. 
In carcinoma cancer cells, over-expression of MMP-14 has been also associated with epithelial to mesenchymal transition (EMT), a highly conserved and fundamental process of morphological transition (Egeblad and Werb, 2002; Gilles et al., 1996). In particular, during this event, epithelial cells actively down-regulate cell-cell adhesion systems, lose their polarity, and acquire a mesenchymal phenotype with reduced intercellular interactions and increased migratory capacity (Polyak and Weinberg, 2009).

MT1-MMP shares with MT3-MMP and MT5-MMP the capability of activating MMP-2 and MMP-9 providing a mechanism of control in cancer. These type of activation induce the inactivation of the human metastasis suppressor gene KiSS-1produced by cancer cells (Takino et al., 2003).

7.2.2.3. Angiogenesis. The key players of the MMP family that participate in tumor angiogenesis are mainly MMP-2, MMP-9 and MMP-14 (Rundhaug, 2003). Initially, the generation of cryptic peptides via degradation of ECM molecules, such as collagen type IV and laminin-5, promotes the migration of cancer cells (Koshikawa et al., 2000; Xu et al., 2001). Laminin-5 is a major component of the basement membrane, which regulates the function of epithelial cells. Several cancer breast epithelial cell lines constitutively express MT1-MMP and generate a unique fragment derived from the cryptic site of the c2-chain of laminin-5, directly affecting the cellular signal and promoting cell migration (Gilles et al., 2001; Koshikawa et al., 2000).The generation of a fragment from laminin-5 c2-chain by MT1-MMP in human uveal melanoma cells plays critical roles in the vasculogenic mimicry (Seftor et al., 2001).

MT1-MMP may modulate the ability of cancer cells to induce angiogenesis. Tumor cells expressing MT1-MMP grow rapidly and form highly vascularized tumors (Hotary et al., 2003; Sounni et al., 2002b). The proangiogenic activity of MT1-MMP is further supported by the effects of MT1-MMP overexpression on tumorigenicity, growth and vascularization of human melanoma (Maquoi et al., 2004; Sounni et al., 2002b), breast adenocarcinoma (Sounni et al., 2002a) or glioma (Deryugina et al., 2002b) cells transfected with MT1-MMP cDNA. Other studies provided evidence that this MT1-MMP pro-angiogenic effect is mediated at least by an up-regulation of VEGF at both mRNA and protein levels by activation of signaling pathway involving Src type tyrosine kinases (Sounni et al., 2002a, 2004). However, the expression of other angiogenic factors, such as VEGF-B, -C, -D, PIGF, angiopoietin or their receptors VEGF-R1, -R2, neuropilin, Tie-1 and Tie-2 is not affected by MT1-MMP over-expression (Sounni et al., 2004). Such a link between the expression of VEGF and MT1-MMP is further confirmed by immunohistochemical and RT-PCR analysis of human glioma tissue samples (Munaut et al., 2002). Correlation between MT1-MMP and VEGF-A has also been observed in early physiological cartilage formation (Sekiya et al., 2002).

MT-MMPs play important roles in the formation of new blood vessels in both physiological and pathological conditions. The activities of MT1-MMP, MT2-MMP, MT3-MMP and MT5-MMP are essential for microendothelial cell differentiation into tubes in a fibrin gel (Lafleur et al., 2002). TIMP-2 and TIMP-4, but not TIMP-1, are able to inhibit angiogenesis in vitro (Lafleur et al., 2002). In the absence of uPA/plasminogen system, MT1-MMP fibrinolytic activity supports the tubule formation of endothelial cells in a fibrin gel (Hiraoka and Furukawa, 1998). In 3D-collagen gel, expressions of MT1-MMP and MT3-MMP are required for MDCK and endothelial cell organization into a tubular network (Haas et al., 1998; Kadono et al., 1998; Kang et al., 2001). Although embryonic vasculogenesis and angiogenesis are normal in MT1-MMP-deficient mice, the formation of secondary ossification centers during bone formation is severely impaired due to defect of angiogenesis (Zhou et al., 2000).

Angiogenesis is severely impaired in an ex vivo assay using cornea issued from MT1-MMP-deficient mice, as compared to wild-type mice (Zhou et al., 2000). Corneal epithelial MT1-MMP displays an antiangiogenic activity, which does not require the catalytic domain (Azar et al., 2010). On the other hand, (MT1-MMP), acts as proangiogenic enzyme, cleaving an antiangiogenic protein named decorin (Mimura et al., 2009).

The control of VEGF bioactivity by MMPs has also been reported. The effects of synthetic MMP inhibitors on the expression of VEGF in MCF7 overexpressing MT1-MMP and in an in vivo T-cell lymphoma model, support the role of MT1-MMP in promoting tumor angiogenesis during cancer progression (Arlt et al., 2002; Sounni et al., 2004). MT1-MMP can induce tumor angiogenesis through up-regulation of VEGF expression in human breast carcinoma (Deng et al., 2009).

MMP-14 is a shedding protease for endoglin, a transforming growth factor- $\beta$ coreceptor with a crucial role in angiogenesis. MMP-14 cleaves endoglin at position 586 close to the transmembrane domain, releasing full-length endoglin extracellular domain. Endoglin inhibits angiogenesis and would therefore be a useful tool to inhibit tumor angiogenesis. In turn, inhibiting MMP-14 might be a therapeutic strategy for pathologic conditions with increased endoglin shedding, such as preeclampsia (Hawinkels et al., 2010).

7.2.2.4. Biomarker role. MT1-MMP is a candidate marker to predict distant metastasis among identified genes. MT1-MMP-positive expression in peripheral blood was associated with incidence of peritoneal dissemination, lymphatic permeation, vascular permeation, and lymphonode metastasis. MT1-MMP-positive expression in bone marrow was also significantly related to the incidence of distant metastasis and peritoneal dissemination (Mimori et al., 2008). MT1-MMP plays an important role in the progression of the laryngeal cancer, and may serve as a reliable marker in estimating invasive and metastatic potency of laryngeal cancer (Sun and Li, 2004).

\subsubsection{Non-neoplastic diseases}

7.2.3.1. Diseases of the cardiovascular system. A number of studies have shown that MMP-14 is involved in the remodeling of the myocardium in cardiac diseases including myocardial infarction, left ventricular hypertrophy and dilated cardiomyopathy (Spinale, 2007). MMP-14 is elevated during pressure overload hypertrophy (Spinale, 2007). 
Several studies indicate that MMP-14 collagenolytic activity contributes to collagen remodeling in the atherosclerotic lesion. Lesions of low-density lipoprotein receptor-deficient mice engrafted with MMP-14 knockout bone marrow contained more interstitial collagen than those receiving wild-type bone marrow (Schneider et al., 2008).

MMP-14 has been detected in human abdominal aortic aneurysms, which are lesions with the potential to rupture (Choke et al., 2005; Thompson et al., 2002), accompanied by a MMP-14 over-expression (Annabi et al., 2002). Elevated transplantation with bone marrow from MMP-14 null mice resulted in a resistance to aortic aneurysm formation, a finding which supports the importance of MT1-MMP/TIMP-2-driven proMMP-2 activation in aneurysm formation (Xiong et al., 2009).

7.2.3.2. Diseases of the nervous system. In the nervous system, relatively high mRNA and protein levels of MMP-14, MMP-15, and MMP-24 and TIMP-1 and TIMP-3 are found in the perinatal rodent central nervous system (CNS) but generally decline with age (Ayoub et al., 2005; Fager and Jaworski, 2000; Jaworski, 2000; Ranasinghe et al., 2009; Rivera et al., 1997; Ulrich et al., 2005; Vaillant et al., 1999).

Cerebral ischemia and hemorrhagy initiates a complex pattern of MMP expression that is important in both the injury and repair. Shortly after the initiation of an ischemic damage in animals, basal lamina disruption is observed (Hamann et al., 1995; Heo et al., 1999). Because MMPs are present in latent forms, mechanisms of activation must be brought into play. With the onset of oxygen deprivation, hypoxia inducible factor-1 (HIF-1) activates genes that are important in adapting the brain to the hypoxic conditions, including genes involved in anaerobic metabolism, angiogenesis, red blood cell production, and growth factor production. Notably, HIF-1 activates the proprotein convertase furin, which activates MMP-14, with the consequent formation of a trimolecular complex between MMP-14, TIMP-2, and pro-MMP-2, followed by the formation of active MMP-2. Thus, the initial phase of BBB disruption is mainly mediated by MMP-14. Ultimately, this combination affects the basal lamina and the tight junction proteins claudin and occludin (Furuse et al., 1998; Hawkins and Davis, 2005). After initial structural changes in tight junction proteins, there is resolution of blood-brain barrier (BBB) opening within several hours in the ischemic/reperfusion suture model (Yang et al., 2007a).

7.2.3.3. Diseases of joints and muscular system. Among the MMPs, MMP-13, MMP-9 and MMP-14 (MTl-MMP) appear to be the most important in regulating cellular migration, ECM protein transformation, ECM degradation and apoptosis in the growth plate (Werb and Chin, 1998). Although normal endochondral ossification is also dependent to a significant extent on the activities of members of the hedgehog protein family, parathyroid hormone-related peptide and parathyroid hormonerelated peptide receptor (Kobayashi et al., 2002) as well as specific stress-activated and tyrosine receptor protein kinases (Wang et al., 2004b), ablation of the MT1-MMP (Holmbeck et al., 1999) gene to produce MMP-specific null mice resulted in space-temporal modulation of growth plate development.

Accumulated lines of evidence have demonstrated that collagenolytic MMP (MMP-14) is responsible for the degradation of type II collagen (a major fibrillar collagen in the cartilage). In human osteoarthritis (OA) cartilage, MMP-1, MMP-8, MMP13 and MMP-14 are overexpressed by chondrocytes (Okada, 2009). Among these MMPs, MMP-14 may play a direct role in collagen degradation because of its collagenolytic activity and common expression in the cartilage. Importantly, MMP-14 potentially has a dual role in collagen degradation through both its collagenolytic activity and activation of pro-MMP-2 and pro-MMP-13.

The mechanism of articular cartilage destruction by proteinases is more complex in rheumatoid arthritis (RA) than OA, and composed of three pathways: destruction from the surface of the articular cartilage by proteinases present in the synovial fluid, destruction through direct contact of proteolytic synovium and/or pannus tissue to the articular cartilage and intrinsic destruction by proteinases derived from chondrocytes (Okada, 2009). Rheumatoid synovial tissue exhibits the overproduction of MMP-1, MMP-3, MMP-8, and MMP-14 together with TIMP-1, TIMP-2 and TIMP-3 (Okada, 2009).

Rheumatoid synovium is highly proteolytic, since it has additional activity generated by the activation of proMMP-2 by MMP-14, This activity was blocked when incubated with BB94, a specific MMP inhibitor (Yamanaka et al., 2000). MT1-MMP is expressed in all rheumatoid synovial tissue. Significant correlation is found between the mRNA expression level of MT1MMP and the activation ratio of proMMP-2 $(p<0.01)$. In situ hybridization indicated that the hyperplastic lining cells of rheumatoid synovium express MT1-MMP. Immunohistochemistry demonstrated that MT1-MMP was co-localized with MMP-2 and with a tissue inhibitor of metalloproteinase-2, and was mainly located in the rheumatoid synovial lining cells (Yamanaka et al., 2000). Direct contact of this synovium may induce cartilage destruction at the peripheral portion of articular surface (Yamanaka et al., 2000). Chondrocytes in RA also express MMP-1, 13, MT1-MMP and MT3-MMP.

7.2.3.4. Wound healing. Wound tissues in humans and in experimental animals over-express multiple MMP including MMP14. These MMPs expectedly play a role in the complex process of wound healing that includes acute inflammatory reaction, regeneration of parenchyma cells, cell migration and proliferation, angiogenesis, contraction and tissue remodeling (Gill and Parks, 2008). In particular, MMP-14 may contribute to re-epithelialization (Gill and Parks, 2008).

7.2.3.5. Diseases of the respiratory system. Membrane type 1 matrix metalloproteinase (MT1-MMP) expressed by airway epithelial cells is implicated in bronchial epithelial repair, involving keratinocyte growth factor receptor (KGFR) expression and epithelial cell proliferation after acute airway injury (Atkinson et al., 2007).

Induced mainly by cigarette smoking, chronic obstructive pulmonary disease (COPD) is a global public health problem characterized by progressive difficulty in breathing and increased mucin production. MMP-14 together with MMP-9 activity 
increases in COPD sputum; in particular, MMP-14 expression is induced in mucin production in COPD (Deshmukh et al., 2009).

\subsection{MT-2 MMP (MMP-15)}

\subsubsection{Structure and activation}

The insertion sequence-2 (IS-2) is functionally important, since transfection of the mouse membrane type-2 matrix metalloproteinase (MT2-MMP) gene into COS-1 cells resulted in the activation of progelatinase A; on the other hand, the same event did not produce any effect on the human gene. In particular, seven amino acid residues of the IS-2 (located between amino acid residues 155 and 271) are not conserved between human and mouse MT2-MMP (Miyamori et al., 2000). However, MT2-MMP has been demonstrated to be able to activate pro-MMP-2 in vitro (Sato et al., 1994).

\subsubsection{Biological features}

MT1-MMP and MT2-MMP are predominantly expressed in human placenta during the first trimester of pregnancy (Bjørn et al., 2000), exerting essential but interchangeable roles in placental vasculogenesis. The fetal portion of the placenta, in particular the labyrinth (LA), displays strong overlapping expression of MT1-MMP and MT2-MMP, which is critical for syncytiotrophoblast formation and for fetal vessels. Both MT1-MMP and MT2-MMP are crucial specifically during development of LA, showing a selective temporal and spatial MMP activity required for the development of the mouse embryo (Szabova et al., 2010). The trophoblast compartment of the placenta comprises various subpopulations with distinct functions. In addition, the first trimester cells of human trophoblast subpopulations-TNF- $\alpha$ stimulate MMP-15 expression (Hiden et al., 2007).

\subsubsection{Neoplastic diseases}

Although MT2-MMP expression in tumors has been found in cervical, breast, head, prostate, and neck cancer, glioblastoma, melanoma, lung adenocarcinoma and astrocytic cancers (Gilles et al., 1997; Giricz et al., 2010; Kobayashi et al., 2004; Nakada et al., 1999; Nuttall et al., 2003; Polette et al., 1996; Riddick et al., 2005), its expression has not been linked to disease progression (Sato and Seiki, 1996). Cancer experimental models have demonstrated that MT1-MMP is negatively regulated by MT2-MMP (Velasco-Loyden et al., 2004). Such a negative regulation of MT1-MMP may explain the lack of correlation between MT2-MMP expression and the aggressiveness of cancers (Velasco-Loyden et al., 2004).

In hepatocellular carcinomas, MT2-MMP displays a pivotal role in the MMP-2 activation process (Théret et al., 1998). Activation of matrix metalloproteinase-2 (MMP-2) by the membrane-type matrix metalloproteinases (MT-MMPs) has been associated with tumor progression. The MT2-MMP and MT3-MMP are MMP-2 activators in pancreatic tumor cell invasion and the development of the desmoplastic reaction characteristic of pancreatic cancer tissues (Ellenrieder et al., 2000), as well as between MMP-2 activity and MT2-MMP expression $(p=0.018)$. There is also an association between MT1-MMP and MT2MMP expression in tumor tissues $(p<0.001)$. MMP-2 activity and MT2-MMP expression in tumor tissues are statistically associated with high tumor stage ( $p=0.039$ and $p=0.014$, respectively), while the MMP-2 activity is associated with an increase in MT2-MMP expression and with lymph node metastasis (Mohammad et al., 2010).

Double immunofluorescence demonstrated a consistent colocalization of MT2-MMP/MMP-2 and MT3-MMP/MMP-2 in the nodular melanoma (NM) and metastatic melanoma cells. The colocalization of MT2,3-MMP and MMP-2 in nodular and metastatic melanoma cells suggests that MT-MMPs and MMP-2 co-operate in the invasive and metastatic process of melanoma cells (Ohnishi et al., 2001).

MT2-MMP is an effector of CXCR4 signaling in glioma cells, and they reveal the novel role of MT2-MMP in modulating tumor growth and metastasis (Zhang et al., 2005b). Significant elevations in the levels of mRNA expression of the matrix metalloproteases (MMPs) membrane type 1-MMP (MT1-MMP), MT2-MMP and MMP-9, as well as of TIMP-2 were detected in the muscle invasive transitional cell carcinoma (TCC) of the bladder (Chaffer et al., 2005).

In the mouse model of endometrial cancer, the commercial drug Avastin, a humanized antibody against vascular endothelial growth factor VEGFA, brings about a down-regulation of MMP-15 transcripts (Davies et al., 2011). The MMP-15 gene is identified as being commonly over-expressed in lung adenocarcinoma (AdC) cells in comparison with noncancerous peripheral lung epithelial cells, type II alveolar cells and bronchiolar epithelial cells. This strongly suggest that the MMP15 genes could be a novel marker for molecular diagnosis and therapy of lung AdC (Kobayashi et al., 2004). Increased expression of MMP-15 in malignant tissue, as compared to benign prostate tissue, is detected suggesting that MMP-15 gene involved in proteolysis can be a potential prognostic indicator (Riddick et al., 2005).

A crucial step in human breast cancer progression is the acquisition of invasiveness. The expression of matrix metalloproteinases MMP-15, and MMP-17 is up-regulated in the invasive cells. Small interfering RNA-based data demonstrated that MMP-15 and MMP-17 are required for the invasive phenotype of breast cells (Rizki et al., 2008).

7.3.3.1. Angiogenesis. The intensity of immunochemical staining of MT2-MMP was significantly correlated in a positive fashion to the intratumoral angiogenesis of esophageal cancer tissues. In addition, MT2-MMP immunochemical intensities were significantly correlated to tumor size, but not to patient's gender, age, invasion depth, lymphonode metastasis and distant metastasis. Moreover, MT2-MMP levels could not be applied for predicting patients' survival rate, although the H-score cut-off value showed the overall survival rate of patients with low MT2-MMP protein level to be better than those with high MT2-MMP protein level (Chen et al., 2010). 


\subsubsection{Non neoplastic diseases}

7.3.4.1. Diseases of the digestive system. In the inflammation processes of the bile ducts, up-regulation of MMP- 15 gene transcription contributes to the pathogenesis of liver tissue during chronic cholangitis disease (Nakken et al., 2007). MMP-15 gene is also significantly up-regulated after hepatic ischemia reperfusion lung injury (Zhao et al., 2009).

\subsection{MT3-MMP (MMP-16)}

\subsubsection{Structure and activation}

The structure of the catalytic domain (CD) of MT3-MMP/MMP-16 in complex with the hydroxamic acid inhibitor batimastat exhibits a classical MMP-fold with similarity to MT1-MMP (see Fig. 1 and Table 2). Nevertheless, it also shows unique properties, such as a modified MT-specific loop and a closed S1' specificity pocket, which might help to design specific inhibitors (Lang et al., 2004).

\subsubsection{Biological features}

Both MT3-MMP and its transmembrane domainless variant MT3-MMP-del hydrolyze gelatin and casein, indicating their broad substrate specificity. The transfection of rat MT3-MMP-del efficiently converts progelatinase A to the intermediate form but not to the mature one, indicating that the transmembrane domain is important for the complete processing of progelatinase A to maturation. Results of experiments with a synthetic MMP inhibitor suggests that MT3-MMP-del and MT3MMP are rapidly degraded immediately after maturation. The multiple forms of MMPs including MT3-MMP are involved in the matrix remodeling of blood vessels (Shofuda et al., 1997).

Inhibitor profile and MMP-16 mutant enzyme studies indicate that MT3-MMP is regulated on the cell surface by the autocatalytic processing and the ectodomain shedding (Zhao et al., 2004). MT1-MMP and MT3-MMP can process the membraneanchored proteoglycan betaglycan (sBG-120) that binds transforming growth factor- $\beta$ (TGF- $\beta$ ) via its core protein, generating a 90-kDa fragment (sBG-90). Notably, the cleavage appears to be mediated by a protease regulated by TIMP-2 (VelascoLoyden et al., 2004). Shedding of the transmembrane heparan sulfate proteoglycan syndecan-1 is not only produced by MT3-MMP but also induced by MT1-MMP, whereas other MT-MMPs are not able to carry out this process. Both shedding processes occur through the preferential cleavage of Gly245-Leu246 peptide bond stimulates cell migration (Endo et al., 2003).

Inhibition kinetic studies showed that TIMP-3 (see Table 5) is a high affinity inhibitor of MT3-MMP as compared with MT1-MMP (with a $K_{\mathrm{i}}=0.008 \mathrm{~nm}$ for MT3-MMP and a $K_{\mathrm{i}}=0.16 \mathrm{~nm}$ for MT1-MMP); conversely, TIMP-2 (see Table 5) is a better inhibitor of MT1-MMP. MT3-MMP requires TIMP-2 to accomplish full pro-MMP-2 activation and this process is enhanced in marimastat pretreated cells, consistent with regulation of active enzyme turnover by synthetic MMP inhibitors. TIMP-3 also enhances the activation of pro-MMP-2 by MT3-MMP but not by MT1-MMP. On the other hand, TIMP-4 cannot support pro-MMP-2 activation with either enzyme (Zhao et al., 2004). Pro-MMP-2 can assemble trimolecular complexes with a catalytic domain of MT3-MMP and TIMP-2 or TIMP-3 suggesting that pro-MMP-2 activation by MT3-MMP involves ternary complex formation on the cell surface. TIMP-3 is a major regulator of MT3-MMP activity and further underscores the unique interactions of TIMPs with MT-MMPs in the control of pericellular proteolysis (Zhao et al., 2004).

MMP-16 plays a roles in cartilaginous embryogenesis as a terminal marker induced by responses to Wnt/beta-catenin signaling. Studies have suggested that continuous Wnt/beta-catenin signaling in nascent cartilaginous skeletal elements blocks chondrocyte hypertrophy and endochondral ossification, whereas signaling starting at later stages stimulates hypertrophy and ossification, indicating that Wnt/beta-catenin roles are developmentally regulated (Tamamura et al., 2005).

\subsubsection{Neoplastic diseases}

Activation of pro-MMP-2 by the membrane-type matrix metalloproteinases (MT-MMPs) has been associated with tumor progression (Ellenrieder et al., 2000). MT3-MMP has been detected in breast and renal carcinomas, astrocytic tumors and melanoma (Kitagawa et al., 1999; Nakada et al., 1999; Takino et al., 1995; Ueno et al., 1997).

MT1, MT2, and MT3-MMP play an important role in the progression of the laryngeal cancer. Early suppression of the expressions of MT1, MT2, and MT3-MMP may inhibit the invasion and metastases of the laryngeal cancer (Sun and Li, 2004).

MicroRNAs (miRNAs) are a class of endogenous, small non-protein coding single-stranded RNA molecules, which are crucial post-transcriptional regulators of gene expression. Using miRNA microarray, miR-146b was identified as one of the miRNAs that is significantly dysregulated in human glioblastoma tissue. The miR-146b molecule is capable of inhibiting glioma cell migration and invasion by targeting MMP-16, underlying a relevant role by MT3-MMP in this tumor (Xia et al., 2009). A different molecule, named miR-146b-5p, by targeting MMP-16 significantly reduces the migration and invasion by MIA PaCa-2 pancreatic cancer cells. This molecule may be a potential therapeutic target for the pancreatic cancer (Lin et al., 2011b).

MT3-MMP possesses the potential to promote melanoma invasion and proteolysis and the formation of a complex between MT3-MMP and Melanoma chondroitin sulfate proteoglycan (MCSP) may be a crucial step in activating invasion (Lida et al., 2001).

Unlike for other carcinoma, the down-regulation of most MT-MMPs, mainly in epithelial cells. is typical for prostate carcinoma. In malignant tissue samples and their corresponding cell cultures, the expression of most MT-MMPs is down-regulated in comparison to the normal counterparts. There is no correlation between prostate tumor classification data and the MT-MMP expression results (Jung et al., 2003). 
Unexpectedly, several cancer progression modulators located upstream of MMP mRNA expression in human breast cancer cells, induce a decrease of MMP-16 mRNA expression, suggesting a signaling at a late stage in MMP-16 mRNA accumulation, such as MMP-16 mRNA stabilization or late mRNA processing (Delassus et al., 2011).

7.4.3.1. Angiogenesis. In the endometrium, angiogenesis is a physiological process, whereas in most adult tissues neovascularization is initiated only during tissue repair or pathological conditions. Pericellular proteolysis by MT-MMPs plays an important role in angiogenesis being required for endothelial cell migration, invasion, and tube formation. MT1-, MT3-, and MT4-MMPs are expressed in human endometrial microvascular endothelial cells (hEMVECs) and foreskin MVECs (hFMVECs). Immunohistochemistry data have shown that the presence of MT3-MMP in endothelial cells of endometrial tissue. In contrast to tube formation by foreskin MVECs (hFMVECs), which largely depends on MT1-MMP, capillary-like tube formation by hEMVECs is, at least in part, regulated by MT3-MMP (Plaisier et al., 2004).

7.4.3.2. Biomarker role. Microarray analysis of renal cell carcinoma showed an over-expression of MMP-16 in metastatic primary tumors, suggesting that MMP-16 is one of the potential biomarker for metastatic clear cell renal cell carcinoma (Sanjmyatav et al., 2011).

\subsubsection{Non-neoplastic diseases}

7.4.4.1. Diseases of joints and muscular system. Matrix degradation by metalloproteinases is considered a key feature in the loss of articular cartilage seen in many joint diseases. The comparison of the expression of gene of MT-MMP subfamily in normal cartilage and cartilage from patients with osteoarthritis (OA) showed increased expression of MMP-16, which can be a potential specific target to refine the specificity of antiproteolytic therapies (Kevorkian et al., 2004).

However, MT3-MMP expression is elevated in human cartilage at the end-stage osteoarthritis, whereas it is not significantly altered in both osteoarthritis and osteochondrosis cartilage. In addition, gene expression is significantly downregulated by the addition of recombinant human interleukin- $1 \beta$, oncostatin $M$, or tumor necrosis factor- $\alpha$ to normal cartilage explants (Garvican et al., 2008).

The expression of MT1-MMP, MT2-MMP, and MT3-MMP, and proMMP-2 production levels and activation ratios are found to be significantly higher in rheumatoid synovium, as compared with normal synovium $(p<0.01)$. supporting a positive correlation between the levels of MT-MMPs and the extent of proMMP-2 activation in rheumatoid synovial tissue (Yamanaka et al., 2000). Similarly to MT1-MMP, MT3-MMP is expressed in all rheumatoid synovial tissue, but the mean expression level of MT3-MMP is approximately 11-fold lower than that of MT1-MMP. In situ zymography of the rheumatoid synovium showed gelatinolytic activity, predominantly in the lining cell layer (Yamanaka et al., 2000).

\subsection{MT5-MMP (MMP-24)}

\subsubsection{Biological features}

In contrast to other MT-MMPs, MT5-MMP tends to shed from cell surface as a soluble enzyme, thus envisaging the possibility to work in extracellular matrix remodeling processes both as a cell bound and soluble proteinase (Pei, 1999a).

MT5-MMP can degrade several ECM components, such as inhibitory chondroitin sulfate proteoglycans, thereby promoting neurite outgrowth in vitro (Bar-Or et al., 2003; Hayashita-Kinoh et al., 2001; Jaworski, 2000; Wang et al., 1999a). MT5-MMP can degrade purified proteoglycans and gelatin substrates in vitro (Wang et al., 1999a) and can also relieve the inhibitory function of proteoglycans during axonal elongation on laminin-coated plates (Hayashita-Kinoh et al., 2001). Moreover, it appears capable of mediating the cleavage of cell-adhesion molecule $\mathrm{N}$-cadherin in heterologous cells (Monea et al., 2006).

MT5-MMP (MMP-24) is mainly expressed in neuronal cells of both central and peripheral nervous systems, although its presence has been also detected in inflammatory cells (Bar-Or et al., 2003; Hayashita-Kinoh et al., 2001; Jaworski, 2000; Llano et al., 1999; Pei, 1999a).

In the cerebellum, its expression was regulated developmentally and is closely associated with dendritic tree formation of Purkinje cells, suggesting that MT5-MMP may contribute to neuronal development (Sekine-Aizawa et al., 2001).

MT5-MMP expression is ubiquitous during the brain development while in adult it is restricted to hippocampus and cerebellum regions, displaying synaptic plasticity (Ross and Fillmore, 2007). More than one human MT5-MMP transcript may exist in the central nervous system (Monea et al., 2006; Ross and Fillmore, 2007).

MMP-24 contributes to synaptogenesis and activity-dependent synaptic remodeling, since AMPA receptor binding protein (ABP) and glutamate receptor interacting protein (GRIP) spatially regulate MT5-MMP localization and hence its degradation of ECM or cell adhesion molecules (Monea et al., 2006).

MMP-24 is an essential mediator of peripheral thermal nociception and inflammatory hyperalgesia, mediating the neuroimmune communication. Lopez-Otin and colleagues have demonstrated that the absence of MT5-MMP induces a cutaneous phenotype of hyperinnervation and a morphological and functional alteration in neuro-immune interactions (Folgueras et al., 2010). In contrast to basal nociception, MMP-24(-/-) mice do not develop thermal hyperalgesia during inflammation, a phenotype that appears associated with alterations in $\mathrm{N}$-cadherin-mediated cell-cell interactions between mast cells and sensory fibers (Folgueras et al., 2010) (see Table 6). Therefore, MT5-MMP plays an essential role of in the development of dermal neuro-immune synapses and may be a target for pain control (Folgueras et al., 2010). 
MT5-MMP is expressed by CGRP-containing peptidergic nociceptors in dorsal root ganglia and MMP-24-deficient mice display enhanced sensitivity to noxious thermal stimuli under basal conditions. Consistently, mutant peptidergic sensory neurons hyperinnervate the skin, a phenotype that correlates with changes in the regulated cleavage of the cell-cell adhesion molecule N-cadherin (Fariñas and Lopetz-Otin, 2009).

\subsubsection{Neoplastic diseases}

MMP-24 gene silencing by RNAi demonstrates that MMP-24 can suppress the invasiveness of ovarian cancer SKOV cells in vitro, which may provide a new therapeutic approach of ovarian cancer (Luo et al., 2009).

\subsubsection{Non-neoplastic diseases}

7.5.3.1. Diseases of the nervous system. MMP-24 is expressed in brain tissue after the development and colocalization with senile plaques in Alzheimer brain indicates possible roles in neuronal remodeling naturally occurring in adulthood and in regulating pathophysiological processes associated with advanced age (Sekine-Aizawa et al., 2001). MT5-MMP expressed in neurons may play a role in axonal growth that contributes to the regulation of neural network formation (Hayashita-Kinoh et al., 2001) and may promote structural remodeling after nerve injury (Komori et al., 2004).

7.5.3.2. Other diseases. The evidence of the overexpression of a MT5-MMP in diabetes suggests a role for this enzyme in the pathogenesis of renal tubular atrophy and end-stage renal disease (Romanic et al., 2001). MT5-MMP expression was localized by immunohistochemistry into the epithelial cells of the proximal and distal tubules, the collecting duct, and the loop of Henle. Furthermore, the tubular epithelial cells that expressed MT5-MMP are associated with tubular atrophy. Because renal tubular atrophy is a significant factor in the pathogenesis of diabetic nephropathy and renal failure, although the molecular mechanisms regulating this process remain unknown, it is hypothesized that the elevated expression of MT5-MMP contributes to the activation of pro-MMP-2, which participates in the remodeling of the proximal and distal tubules as well as in the collecting duct (Romanic et al., 2001).

Along with MMP-1, MMP-2, MMP-3, MMP-7, MMP-13, also MT5-MMP participates into the early phases in endometriotic lesion formation (Nap et al., 2004). Both the gene chip expression and PCR analyses indicated expression of MT5-MMP in normal human endometrium and strongly elevated transcript levels in most peritoneal endometriosis lesions analyzed. Moreover an enhanced MT5-MMP expression in the eutopic endometrium from patients suffering from endometriosis was detected, further supporting a role of MT5-MMP in the formation of endometriosis (Gaetje et al., 2007).

\subsection{GPI-MT-MMPs: MT4-MMP (MMP-17) and MT6-MMP (MMP-25)}

\subsubsection{GPI-MT-MMP structure}

The GPI-MT-MMPs subfamily includes MT4-MMP (MMP-17) (Kajita et al., 1999; Puente et al., 1996) and MT6-MMP (MMP-25) (Pei, 1999b; Velasco et al., 2000). The two members of the GPI-MT-MMP subfamily are anchored to the plasma membrane via a glycosylphosphatidylinositol (GPI) anchor, which confers these enzymes a unique set of regulatory and functional mechanisms that distinguishes them from the rest of the MMP family (Sohail et al., 2008) (see Fig. 2 and Table 2).

GPI-MT-MMPs are structurally and functionally distant from TM-MT-MMPs. Primary sequence alignment of the catalytic domain of MT4- and MT6-MMP shows they are 56\% identical and 77\% homologous. However, when compared to the MT1MMP catalytic domain, MT4-MMP displays only 37\% identity (50\% similarity) while MT6-MMP possesses $42 \%$ identity (58\% similarity).

With the exception of the anchoring region, GPI-MT-MMPs share the same basic domain organization of most MMPs, including a signal sequence, a propeptide domain, a zinc-containing catalytic domain, a hinge region (linker 1 ), and a hemopexin-like domain (see Fig. 1). Both the transmembrane and the GPI-MT-MMPs also contain a RXR/KR motif at the end of the propeptide domain that serves as a recognition site for pro-convertases, such as furin, which cleave the propeptide and activate the MTMMP zymogen (see Fig. 1).

On the other hand, the hemopexin-like domain of the GPI-MT-MMPs is followed by a hydrophilic region ( $\sim 35-45$ amino acids), also called stem (linker 2) region, and by a hydrophobic tail of $\sim 20-23$ amino acids. The C-terminal hydrophobic tail of the GPI-MT-MMPs is removed by a transamidase in the endoplasmic reticulum (ER) during incorporation of the GPI anchor, a process that is a unique characteristic of GPI-anchored proteins (Udenfriend and Kodukula, 1995).

\subsubsection{MT4-MMP (MMP-17)}

MT4-MMP was identified in peripheral blood leukocyte preparations and was first cloned from a human breast carcinoma cDNA library (Puente et al., 1996).

7.6.2.1. Structure. MT4-MMP is displayed on the cell surface as a mixed population of monomeric, dimeric, and oligomeric forms. Sucrose gradient fractionation experiments demonstrated that these forms of MT4-MMP are all present in lipid rafts. Mutational and computational analyses revealed that Cys564, which is present within the stem region, mediates MT4-MMP homodimerization by forming a disulfide bond. Substitution of Cys564 results in a more rapid MT4-MMP turnover, when compared to the wild-type enzyme, consistent with a role for dimerization in protein stability. Expression of MT4-MMP in MDCK cells enhanced cell migration and invasion of Matrigel, a process that requires catalytic activity. However, a serine 
substitution at Cys564 did not reduce MT4-MMP-stimulated cell invasion of Matrigel suggesting that homodimerization is not required for this process. Deglycosylation studies also show that MT4-MMP is modified by N-glycosylation (Sohail et al., 2011). Moreover, inhibition of N-glycosylation by tunicamycin diminished the extent of MT4-MMP dimerization, suggesting that N-glycans may confer stability to the dimeric form (Sohail et al., 2011).

7.6.2.2. Biological features. The constitutive expression of MMP-17 mRNA is detected in unstimulated eosinophils, lymphocytes, and monocytes (but not in neutrophils) and its expression is modulated by TNF- $\alpha$ in purified human blood eosinophils Consequently, MT4-MMP may be directly involved in the degradation of extracellular matrix components and/or in the modulation of the activity of other proteins implicated in eosinophil migration and tissue remodeling (Gauthier et al., 2003).

MT4-MMP exhibits minimal or no activity against many ECM proteins (see Table 3). Specifically, soluble MT4-MMP was found to be active only against gelatin, fibrin, and fibrinogen (English et al., 2000; Kolkenbrock et al., 1999; Rozanov et al., 2004; Wang et al., 1999b). As a matter of fact, MT4-MMP has been observed to display ADAM-17-like activity (see Section 1.1.2), since it can behave as a sheddase of tumor necrosis factor (TNF)- $\alpha$ when co-transfected with pro-TNF- $\alpha$ in Cos-7 cells (English et al., 2000). The MT4-MMP catalytic domain alone cleaves $\alpha 2$-macroglobulin (English et al., 2000) and low density lipoprotein receptor related protein (LPR) (Rozanov et al., 2004).

MMP-17 is selectively expressed by distinct subsets of direction-selective retinal ganglion cells, suggesting that it is involved in the molecular mechanisms which regulate the cellular motion in different directions (Kay et al., 2011).

7.6.2.3. Neoplastic diseases. MT4-MMP mRNA is consistently found to be over-expressed in breast cancer tissues (Chabottaux et al., 2006). On the basis of immunochemistry studies the levels of MT4-MMP were observed to be particularly high in metastatic lymphonodes where both tumor and inflammatory cells displayed strong staining (Chabottaux et al., 2006). This is consistent with the reported expression of MT4-MMP in leukocytes (Gauthier et al., 2003; Puente et al., 1996). While human breast cancer tissues express MT4-MMP (Chabottaux et al., 2006; Puente et al., 1996), the expression and function of MT4MMP in mouse mammary carcinoma are unclear. Over-expression of recombinant MT4-MMP in the breast cancer cell line MDA-MB-231 enhanced subcutaneous tumor growth when inoculated in RAG-1 immunodeficient mice (Chabottaux et al., 2006).

MT4-MMP mRNA expression was also examined in human gliomas (Nuttall et al., 2003) and it was found to decrease with advancing tumor grade. Studies in brain and breast tumors also suggest that the association of MT4-MMP expression with tumor progression may be cancer type specific. Interestingly, functional studies failed to reveal an effect of MT4-MMP overexpression on in vitro cell migration and invasion, pro-MMP-2 activation, VEGF production, and angiogenesis (Chabottaux et al., 2006).

\subsubsection{Non neoplastic diseases.}

7.6.2.4.1. Diseases of joints and muscular system. Recently, MMP-17 has been implicated in the activation of one of the key aggrecanases ADAMTS4 (see Section 1.1.2), revealing an association between increased levels of MMP-17 expression and inflammatory processes in areas of osteoarthritis cartilage damage (Clements et al., 2011).

7.6.2.4.2. Inflammation. Expression of MT4- and MT5-MMPs was induced in corneas infected with Pseudomonas aeruginosa. Immunohistochemistry showed predominant immunoreactivity of MT4- and MT5-MMPs in the substantia propria. Previous histologic studies have revealed different patterns of inflammatory cell infiltration with an increased number of polymorphonuclear neutrophils (PMNs) during the early stage of inflammation and increased macrophages during the late stage. These results indicate a good correlation between the over-expression of the MT-MMPs in the infected corneas and the inflammatory response, indicating that inflammatory cells, such as macrophages and PMNs, may play a role in the upregulation of MT-MMPs during corneal infection, which in turn can lead to the destruction of corneal tissue (Dong et al., 2001).

\subsubsection{MT6-MMP (MMP-25)}

MT6-MMP was identified from peripheral blood leukocytes and this MMP was also termed leukolysin due to the high expression of MT6-MMP in leukocytes (Pei, 1999b).

7.6.3.1. Structure. The natural MT6-MMP expressed on the cell surface is a major reduction-sensitive form of MW $120 \mathrm{kDa}$, likely representing enzyme homodimers held by disulfide bridges. Among the membrane type-MMPs, the stem region of MT6-MMP contains three cysteine residues at positions 530, 532, and 534 which may contribute to the dimerization interface, which provides structural stability to the protein (Zhao et al., 2008). A site-directed mutagenesis study of the Cys residues in the stem region shows that Cys (532) is involved in MT6-MMP dimerization by forming an intermolecular disulfide bond. The mutagenesis data also suggest that Cys (530) and Cys (534) form an intramolecular disulfide bond. Dimerization is not essential for transport of MT6-MMP to the cell surface, partitioning into lipid rafts or cleavage of alpha-1-proteinase inhibitor. However, monomeric forms of MT6-MMP exhibited enhanced autolysis and metalloprotease-dependent degradation (Zhao et al., 2008).

7.6.3.2. Biological features. MT6-MMP seems to function like stromelysin-1 (see Section 5.1.1), being able to cleave type-IV collagen, gelatin, fibronectin and fibrin (English et al., 2001). However, it differs from stromelysin-1 and MT1-MMP, since it is able to cleave laminin-I and cannot activate progelatinase B (see Table 3). MT6-MMP could play a role in cellular 
migration and invasion of the extracellular matrix and basement membranes and its activity may be tightly regulated by all members of the TIMP family (English et al., 2001).

The regulation mechanisms, the functional role, and the repertoire of physiologically relevant cleavage targets of MT6MMP remain largely unknown. A number of extracellular matrix components can be cleaved, including fibronectin, fibrin, gelatin, type IV collagen, and chondroitin and dermatan sulfate proteoglycan (English et al., 2001; Kang et al., 2001). The catalytic domain of MT6-MMP has been reported to cleave galectin-3, urokinase plasminogen activator receptor chondroitin dermatan sulfate proteoglycans and myelin basic protein (Kang et al., 2001; Radichev et al., 2010; Shiryaev et al., 2009b; Sohail et al., 2008).

Polymorphonuclear neutrophils (PMNs) express high levels of MT6-MMP, suggesting that it plays a role in the physiology of human PMNs. It is present in the membrane, granules and nuclear/endoplasmic reticulum/Golgi fractions of PMNs where it is displayed as a disulfide-linked homodimer of $120 \mathrm{kDa}$. Stimulation of PMNs resulted in secretion of active MT6-MMP into the supernatants. Conversely, membrane-bound MT6-MMP is located in the lipid rafts of resting PMNs and stimulation does not alter this location. In addition, TIMP-2, a natural inhibitor of MT6-MMP, does not co-localize with it in the lipid rafts. Interestingly, living PMNs do not display MT6-MMP on the cell surface. However, induction of apoptosis induces MT6-MMP relocation on PMNs' cell surface (Fortin et al., 2011).

There are conflicting results that point out the potential role of MT6-MMP in the mechanisms of MMP-2 activation (Nie and Pei, 2003; Sohail et al., 2008). Even though MT6-MMP does not stimulate cell migration, it generates however a significant level of gelatinolysis of the fluorescein isothiocyanate-labeled gelatin and exhibits an intrinsic, albeit low, ability to activate MMP-2. Because of its lipid raft localization, cellular MT6-MMP is inefficiently internalized. MT6-MMP is predominantly localized in the cell-to-cell junctions (Radichev et al., 2010).

MMP-25 is also present in the extracts of the developing tendon, suggesting its involvement in fibrillogenesis and matrix assembly (Smith et al., in press).

MMP-25 gene expression is high during the development of mouse secondary palate, wherefore MMP-25 is the only MMP showing a functional role (Brown and Nazarali, 2010).

7.6.3.3. Neoplastic diseases. Elevated MT6-MMP mRNA expression was found in several human cancers including brain (anaplastic astrocytomas and glioblastomas) (Nuttall et al., 2003; Velasco et al., 2000), colon (Velasco et al., 2000), urothelial (Wallard et al., 2006), and prostate (Riddick et al., 2005) cancers. In gliomas, expression of MT6-MMP mRNA was suggested to contribute to the disease progression (Nuttall et al., 2003). However, in vitro studies showed no effects of MT6-MMP overexpression on tumor cell migration and invasion, and therefore the precise role of MT6-MMP in colon cancer remains elusive. These findings are similar to those obtained with MT4-MMP in colon cancer cells, in which the protease expression does not confer a migratory and/or invasive phenotype or ability to activate pro-MMP-2 (Sun et al., 2007).

Although more studies are required to unveil the roles of GPI-MT-MMPs in cancer, the data so far obtained suggest that these proteases influence cancer progression by mechanisms that are different from the TM-MT-MMPs. First, GPI-MTMMPs do not act as progelatinase activators; second, their ECM degradation profile appears to be very limited; third, GPI-MT-MMPs do not promote tumor cell migration and invasion; and fourth, their inhibition profile appears unique.

7.6.3.4. Non-neoplastic diseases. MMP-25 plays a significant role in the fragmentation autoimmune multiple sclerosis (MS). MT6-MMP proteolysis of the myelin basic protein (MBP) isoforms generated the highly immunogenic N-terminal MBP peptide which efficiently stimulated the proliferation of the specific T cell clone isolated from mice with experimental autoimmune encephalomyelitis (EAE) (Shiryaev et al., 2009b).

Furthermore, the depletion of dietary antioxidants in asthma may result in upregulation of MMP-25 (Baines et al., 2009).

Among MMP members MMP-8, MMP-9 and MMP-13 play the most prominent role in the pathogenesis of periodontal disease progression (Giannobile, 2008). Like these MMPs, MMP-25 participates into the progression of the periodontal lesion. Expression of MMP-25 is associated with periodontal and peri-implant inflammation. Immunoblot in myeloma cell lysates reveals 29-57-kDa MMP-25, but not in macrophages, and partly active MMP-25 is observed in inflamed gingival crevicular fluid and peri-implant sulcular fluid (Kuula et al., 2008).

7.6.3.4.1. Biomarker role. MMP-25 is a serum marker for atopic asthma or chronic obstructive pulmonary disease (COPD). Among individuals with history of chronic airways inflammation (asthma and COPD) serum MMP-25 is a metabolic marker associated with chronic atopy-associated respiratory inflammation. Common factors may stimulate increased production or release of both MMP-25 from myeloid cells and IgE from lymphoid cells (Blumenthal et al., 2010).

\section{Other matrix metalloproteinases}

\section{1. $M M P-12$}

\subsubsection{Structure and function}

MMP-12, also called macrophage metalloelastase, is the most active MMP against elastin (Shapiro, 1998). MMP-12 is a $54 \mathrm{kDa}$ proenzyme that is processed into a $45 \mathrm{kDa}$ and then a $22 \mathrm{kDa}$ active forms (Shapiro et al., 1992). The human gene, which is designated human macrophage metalloelastase, produces a 1.8 -kb transcript encoding a 470 -amino acid protein 
that is 64\% identical to the mouse protein (Shapiro et al., 1992). MMP-12 is predominantly expressed in alveolar macrophages, in airway epithelial and smooth muscle cells (Belaaouaj et al., 1995; Shapiro et al., 1992) under the control of other matrix components, such as hyaluronan fragments, cytokines and growth factors (e.g., TGF- $\beta$, IFN- $\gamma$ and EGF), and serine protease, such as thrombin and plasmin (Horton et al., 1999; Raza et al., 2000). A peculiar feature of MMP-12 is that it autocatalytically loses its hemopexin-like domain shortly after activation, apparently without loss of the elastin-degrading function (Curci et al., 1998).

The functional role of MMP-12 appears related to the remodeling of the extracellular matrix in tissues from fetal development to the entire adult life (Kerkela et al., 2001) and it is highly associated with inflammatory diseases involving macrophage infiltration, such as skin diseases (Saarialho-Kere et al., 1999), atherosclerosis (Matsumoto et al., 1998), aneurysms (Curci et al., 1998) and cancers (Kerkela et al., 2000). Moreover, MMP-12 is predominantly involved in acute and chronic pulmonary inflammatory diseases associated with an intense airway remodeling, particularly in the pathogenesis of COPD and emphysema.

Beside elastin, MMP-12 can cleave many of the other components of the extracellular matrix, such as fibronectin, fibrillin1, laminin, entactin, type IV collagen fragments, vitronectin, heparin and chondroitin sulfates (Chen, 2004; Taddese et al., 2010) as well as non-matrix proteins, such as myelin basic protein, tissue factor pathway inhibitor (TFPI) (Belaaouaj et al., 2000), pro-TNF- $\alpha$, (Chandler et al., 1996) plasminogen (Dong et al., 1997) and the blood plasma serpin $\alpha 1$-proteinase inhibitor (Banda et al., 1988) (see Table 3). MMP-12 is also involved in the in vivo activation of other MMPs, such as proMMP-2 and pro-MMP-3, which in turn can activate pro-MMP-1 and pro-MMP-9 (Bhaskaran et al., 2008). Moreover, unlike other MMPs, MMP-12 exhibits the unique property of being able to cleave N-terminal of Arg residues (Gronski et al., 1997).

\subsubsection{Neoplastic diseases}

8.1.2.1. Epidemiological aspects. MMP-12 has been proven to be of relevance for cancers development and prognosis in various organ systems. Increased expression and polymorphisms of MMP-12 have been shown to affect the development of several cancers. In addition, MMP-12 turns out to be the most efficient MMP for producing an endogenous inhibition of endothelial cell proliferation from specific degradation of plasminogen, thus identifying MMP-12 as one of the regulators of angiogenesis and tumor evolution (Shapiro, 1998).

Recently, an association between functional polymorphisms on MMP-12 gene promoter region and epithelial ovarian carcinoma has been described (Jia et al., 2010). MMP-12 genotype is also associated with an elevated esophageal adenocarcinoma (EA) risk and may have prognostic significance in EA patients (Bradbury et al., 2009). Moreover, increased expression of MMP-12 correlates with invasion and differentiation of endometrial adenocarcinoma, epidermal tumors and lung cancer, suggesting an involvement of MMP-12 in tumorigenesis (Hofmann et al., 2005; Kerkela et al., 2000; Yang et al., 2007b).

8.1.2.2. Tumorigenesis. Latest findings report that MMP-12 overexpression in myeloid lineage cells significantly contributes to modulate myelopoiesis, immune suppression and lung tumorigenesis (Qu et al., 2011). Furthermore, human patients affected by Chronic obstructive pulmonary disease (COPD), especially with a smoking history, are a high-risk population for developing lung cancer (Mannino et al., 2003). The molecular mechanism that links COPD and lung cancer is poorly understood, but evidence for an association of polymorphisms of MMP-12 with an higher risk of lung cancer has been provided (Su et al., 2006; Sun et al., 2006). In addition, a comparative genomic hybridization analysis identified in non-small cell lung cancer (NSCLC) an amplified MMP cluster region (11q22) with overexpression of MMP-1, MMP-12 and MMP-13 (Dehan et al., 2007).

The clinical relevance of MMP-12 has been observed in NSCLC, wherefore MMP-12 expression significantly correlates with local recurrence and metastatic disease (Hofmann et al., 2005). Although these studies showed association of MMP12 overexpression with lung cancer, the role of MMP-12 up-regulation remains to be defined. Qu and colleagues tried to delineate the involvement of MMP-12 in the transition process between emphysema (and its associated inflammation) and tumor (Qu et al., 2009). Since MMP-12 overexpression in epithelial cells has been reported in inflammation-triggered lung remodeling, a murine CCSP-rtTA/(tetO)7-MMP-12 bitransgenic model was used. As a result of MMP-12 overexpression, sequential appearance of emphysema, epithelial dysplasia, and bronchioalveolar adenocarcinoma was developed, supporting that MMP-12 acts as a key player that controls emphysema to tumor transition in the lung. This seems partially achieved through the activation of the pro-inflammatory IL-6/Stat3 pathway and inflammatory cell infiltration (Qu et al., 2009).

\subsubsection{Non-neoplastic diseases}

8.1.3.1. Diseases of the cardiovascular system. Data have been accumulating that indicate that MMP-12 gene polymorphisms contribute to inter-individual differences in susceptibility to and outcome of cardiovascular diseases (CVD). Functional polymorphisms in the MMP-12 gene have also been related to coronary artery disease, arterial stiffness, and/or abdominal aortic aneurysm (Jormsjo et al., 2000; Ye, 2006). Furthermore, MMP-12 is up-regulated in atherosclerotic lesions and aneurysms and it may contribute to the activation of other MMPs, which in turn degrade other extracellular matrix proteins (Liang et al., 2006); therefore, this enzyme may represent a potential drug target for CVD treatment and prevention.

In a recent study Tanner and coworkers have evaluated the pharmacogenetic effects of MMP-12 variants on coronary heart disease, stroke, heart failure, combined coronary heart disease and cardiovascular disease. Their data highlight an interaction between antihypertensive drugs and MMP-12 levels for coronary heart disease and composite cardiovascular 
disease, suggesting that the expression of MMP-12 gene may provide useful clinical information with respect to treatment decisions (Tanner et al., 2011).

8.1.3.1.1. Atherosclerosis. Based on histopathological findings, such as the activation of foamy macrophages, the local production of cytokines and chemokines and the involvement of MMPs (Janssens and Lijnen, 2006), atherosclerosis and related diseases are often compared with chronic inflammatory diseases (Hansson and Libby, 2006). The use of animal models strengthens the view that MMP-12 is a player in the hardening and narrowing of the arteries; indeed, MMP-12 knockout mice studies reveal reductions in atherosclerotic lesions size and macrophage number (Johnson et al., 2005) and overexpression of MMP-12 in transgenic rabbits, which induces a stimulation of plaque growth (Liang et al., 2006).

Moreover, Johnson and colleagues have shown that in apolipoprotein E-deficient mouse, inhibition of MMP-12 (using a selective compound) delays atherosclerotic plaque development, thus supporting the hypothesis that MMP-12 is critical for both plaque initiation and progression (Johnson et al., 2008). Recently, Yamada and coworkers have investigated macrophage migration and elastolysis in relation to fatty streaks in human MMP-12 transgenic (hMMP-12 Tg) rabbits, finding that MMP-12 production by macrophages plays a role in the transition from fatty acids to fibrous plaques during the progression of atherosclerosis (Yamada et al., 2008). On the basis of these results a specific MMP-12 inhibitor might eventually turn out to be useful for the treatment of progressive atherosclerosis.

8.1.3.1.2. Aortic aneurysms. MMP-12 has been shown to be upregulated in abdominal aortic aneurysm (AAA), an inflammatory disorder characterized by local connective tissue degradation, macrophage recruitment and infiltration leading to aortic dilation and rupture (Curci et al., 1998). MMP-12 deficiency (see Table 6) attenuates aneurysm growth, possibly by decreasing macrophage recruitment (Longo et al., 2002, 2005). Savio and colleagues support a mechanism whereby MMP-12 inactivation reduces macrophage recruitment into aneurysmal lesion sites by a decrease of proinflammatory cytokines expression and of the consequent vascular wall destruction (Savio et al., 2008). Moreover, recent findings indicate that MMP-12 activity significantly contributes to angiotensin II-induced aneurysm progression in the context of TGF- $\beta$ neutralization and it is critically required for full elastin degradation and vessel rupture (Wang et al., 2010b).

Further, evidence has been provided that deficiency of MMP-12 protects apolipoprotein E-deficient mice against atherosclerotic media degradation and the accompanying ectasia and aortic dilatation (Luttun et al., 2004). However, even in the absence of MMP-12 aneurysms can still form, suggesting that its function is restricted to an enhancement of the elastic fiber degeneration by other MMPs (Longo et al., 2002; Pyo et al., 2000).

8.1.3.2. Diseases of the nervous system. With respect to the CNS, the literature on MMP-12 is sparse. Several studies highlight a linkage between MMP-12 activity and various CNS pathologies.

8.1.3.2.1. Encephalomyelitis. MMP-12 is up-regulated during experimental autoimmune encephalomyelitis and might play an active role during mononuclear cell infiltration in response to CNS infection (Pagenstecher et al., 1998). Several MMPs are increased in experimental autoimmune encephalomyelitis (EAE), an animal model of brain inflammation, with different roles in the pathogenesis. In particular, it was found that MMP-12 plays a protective role by favoring a T-helper 2 (TH2) bias, thereby reducing the TH1 autoimmune response (Weaver et al., 2005).

8.1.3.2.2. Meningitis. Evidence of an association between MMP-12-dependent elastin degradation and the inflammatory reaction of the angiostrongyliasis meningitis has been provides (Wei et al., 2011).

8.1.3.2.3. Multiple sclerosis. Myelin formation is critical for the proper function and survival of axons (Lappe-Siefke et al., 2003). During this event, oligodendrocytes (OLs) mature and they extend processes to contact and enwrap axons (Trapp et al., 1997). The loss of myelin thus results in multiple sclerosis (MS), where demyelination and concurrent axonal loss lead to a relevant disability. It has been reported that MMP-12 is present in active MS lesions in which its expression could be localized into phagocytic macrophages (Vos et al., 2003). In addition, investigation of serum, cerebrospinal fluid and brain tissue of patients with multiple sclerosis has revealed an increase in MMP-12 activity (Kurzepa et al., 2005). MMP-12 is also produced in large amounts by OLs in vitro to regulate their maturation and morphological differentiation (Larsen and Yong, 2004). Many growth factors are involved in the normal development of myelin, among these the insulin-like growth factor 1 (IGF-1) is very important for oligodendrocyte maturation (D'Ercole et al., 2002). IGF binding protein 6 (IGFBP-6), which sequesters IGF-1 and is synthesized in the CNS by astrocytes and OLs (Mewar and McMorris, 1997), is a substrate for MMP-12 processing (see Table 3), suggesting a function for MMP-12 in developmental myelination through the regulation of IGF-1 bioavailability (Larsen et al., 2006).

8.1.3.2.4. Spinal cord injury (SCI). Spinal cord injury (SCI) is a leading cause of permanent disability in young adults, resulting in partial or complete loss of motor and sensory function below the lesion site. Wells and colleagues have evaluated the role of MMP-12 in SCI showing that the expression of MMP-12 is markedly enhanced after mouse spinal cord compression and that MMP-12 null mice have better recovery from SCI than WT controls (Wells et al., 2003).

8.1.3.2.5. Intracerebral hemorrhage. An involvement of MMP-12 in intracerebral hemorrhage (ICH) has also been postulated, since MMP-12 expression has a negative impact on sensorimotor function following intracerebral hemorrhage in mice (Wells et al., 2005). 
8.1.3.3. Disease of joints and muscular system.

8.1.3.3.1. Joints disease. Medial collateral ligament (MCL) injuries heal by a wound repair scar response controlled by a complex cellular and cytokine environment. Many enzymes participate in wound repair, particularly the matrix metalloproteinases. Investigations on MMP-12-deficient and wild-type (WT) mice indicate that MMP-12 is critical in the multifactorial cascade of knee MCL injury healing in both the early inflammatory phase and in tissue repair and remodeling (Wright et al., 2006). Moreover, an involvement of MMP-12 in the etiology of the anterior cruciate ligament (ACL) ruptures has been postulated (September et al., 2007), being also supported by the evidence of an association between the chromosomal region 11q22, in which MMP-12 gene localizes, and the risk of ACL rupture (Posthumus et al., in press).

8.1.3.3.2. Rheumatoid arthritis. Rheumatoid arthritis (RA) is a chronic disease characterized by articular tissue destruction and irreversible joint damage (Firestein, 2003). Ample evidence has shown that in the synovial membrane of RA patients, the number of macrophages is correlated to the extent of joint destruction (Tak et al., 1997). Further, it has been demonstrated that synovial tissue and fluid obtained from patients with rheumatoid arthritis contained higher activity of MMP12 (Liu et al., 2004).

In addition, the effects of overexpressed MMP-12 on the development of experimentally induced inflammatory arthritis has been investigated using human MMP-12 transgenic rabbits which specifically express the human MMP-12 transgene in the macrophage lineage. These results demonstrate that an increased proteolytic activity of MMP-12 magnifies the degradation of the articular connective tissue and cartilage, thus playing a critical role in the development of an inflammatory joint disease (Wang et al., 2004c). Association of MMP-12 genotype with functional disability in rheumatoid arthritis patients was further demonstrated (Ye et al., 2007).

8.1.3.4. Diseases of the respiratory system. The role of MMP-12 is well documented in the pathogenesis of acute and chronic pulmonary inflammatory diseases associated with an intense airway remodeling. Its production stems from macrophage activation but also from resident cells synthesis (i.e., epithelial cells, smooth muscle cells and endothelial cells along alveolar wall of lungs) (Lavigne et al., 2004; Lian et al., 2005).

8.1.3.4.1. Emphysema/chronic obstructive pulmonary disease. Chronic obstructive pulmonary disease (COPD) refers to chronic lung diseases, such as chronic bronchitis (CB), refractory asthma and emphysema, that are generically characterized by progressive airflow limitation and increased shortness of breath (Barnes et al., 2003). COPD represents a worldwide health problem largely attributable to exposure to tobacco smoke (Murray and Lopez, 1997). The pathogenesis of COPD is mainly characterized by an increase in inflammatory cells, such as macrophages, neutrophils and dendritic cells (DCs), in small airways and lung parenchyma (Barnes et al., 2003; Domagala-Kulawik et al., 2003). These cells are all capable of releasing proteinases in excess of their inhibitors, leading to an injury of the extra-cellular matrix (ECM) in the respiratory tract (Van der Vaart et al., 2004).

An essential aspect of COPD and emphysema involving MMP-12 is the degradation of elastin. Elastin is vital for the elastic recoil of the small airways and their ability to resist to a negative pressure collapse. In emphysema, elastin content of the lung parenchyma is decreased and elastic fibers are disorganized and probably nonfunctional (Shapiro, 2000). Moreover, elastin degradation products, such as desmosine, are increased in the urine of subjects with COPD (Stone et al., 1995) and correlate with the rate of lung function decline (Gottlieb et al., 1996).

In vitro studies on alveolar macrophages collected from COPD patients have shown their ability to degrade more elastin than macrophages collected from healthy volunteers (Russell et al., 2002). Furthermore, the resulting elastin fragments are chemotactic for monocytes, resulting in perpetuation of the inflammation (Houghton et al., 2006). Evidence from genetic studies, animal models and human disease linked variations in MMP-12 expression with both lung function in smokers and the risk of developing COPD (Babusyte et al., 2007; Demedts et al., 2006; Hautamaki et al., 1997; Hunninghake et al., 2009). Recently, case-control genetic studies on MMP-12 have given evidence for an association between MMP-12 polymorphisms and severe COPD (Haq et al., 2010, in press).

In several mouse models of COPD, deletion of MMP-12 expression induces resistance to emphysema despite prolonged cigarette-smoke exposure, impairing recruitment of monocytes/macrophages into lung possibly due to the loss of MMP12 mediated generation of elastin fragments (chemotactic for monocytes) (Churg et al., 2002; Hautamaki et al., 1997; Leclerc et al., 2006) (see Table 6). Inflammatory lesions in mouse lung exposed to cigarette smoke is also associated with a significant increase of alveolar macrophages expressing MMP-12 (Valença et al., 2004).

Elevated MMP-12 protein levels have been also detected in the airways of patients affected by COPD. Specifically, MMP12 production by macrophages is significantly increased in sputum (Demedts et al., 2006; LaPan et al., 2010) in bronchoalveolar lavage (BAL) and bronchial biopsies of COPD subjects (Babusyte et al., 2007; Molet et al., 2005).

The macrophage recruitment in lungs and emphysema induced by long-term exposure to cigarette smoke is linked to the elastolytic properties of MMP-12 (Hautamaki et al., 1997). Indeed, it was suggested that elastin degradation took place during development of pulmonary change in mice exposed to cigarette smoke and activation of MMP-12 may be a susceptibility factor for emphysema (Valença et al., 2004). Furthermore, Churg and coworkers have shown that in neutrophils from the bronchoalveolar lavage (BAL) of an acute model of smoke exposure desmosine and hydroxyproline, markers for elastin and collagen breakdown, respectively, cannot be detected in MMP-12 knockout mice; this evidence indeed suggests that acute smoke-induced connective tissue breakdown requires both neutrophils and MMP-12 and that the neutrophil influx is dependent on the presence of MMP-12 (Churg et al., 2002). The mechanism by which MMP-12 triggers cell activation and recruitment associated with inflammatory process in mouse airways involves the induction of TNF- $\alpha$ release from 
macrophages, followed by endothelial cell activation, neutrophil recruitment and secretion of matrix degrading proteases (Churg et al., 2003, 2004). The direct effect of MMP-12 has been also evaluated using a recombinant form of human MMP-12 (rhMMP-12). It was reported that rhMMP-12 catalytic domain, directly injected in the airways of mice, is able to induce an intense inflammatory response characterized by a rapid accumulation of neutrophils correlated to a transient increase in cytokines and chemokines levels (TNF- $\alpha$, MIP- $1 \alpha$, MCP-1, IL-6, and KC) and to gelatinase activation. A later response was also observed mainly due to macrophage recruitment (Nenan et al., 2005). The effect of rhMMP-12 was also examined on human alveolar type II-like epithelial cells (A549) and on human bronchial epithelial cells (BEAS-2B), showing that rhMMP-12 enhances the release of several chemokines by A549 cells, in particular, monocyte chemoattractant protein-1 (MCP-1)/CCL2, growth-related oncogene- $\alpha$ (GRO- $\alpha$ )/CXCL1, and IL-8/CXCL8 (Le Quement et al., 2008). In their study Chen and colleagues have partially elucidated the immunological mechanisms leading to pathologic changes associated to COPD (Chen et al., 2011b). They have shown that cigarette smoke is a powerful T helper 17 [Th17] cells adjuvant and that interleukin 17A receptor [IL-17RA] signaling is required for chemokine expression necessary for MMP-12 induction and tissue emphysema (Chen et al., 2011b).

A protective role for MMP-12 inhibitors has been postulated (Hu et al., 2007; Lagente et al., 2009), since MMP-12 inhibitors can substantially ameliorate morphological emphysema, small airway remodeling and the functional consequences of these lesions in a non-murine species. These findings strengthen the idea that MMP-12 is an important mediator of the anatomical changes behind COPD in humans, identifying MMP-12 as a potential therapeutic target (Churg et al., 2007).

8.1.3.4.2. Bronchial asthma. Asthma is associated with airway inflammation induced by environmental factors, such as allergens, pollutants and respiratory infections. During the course of the disease, an airway remodeling develops that includes epithelial damage, smooth muscle and mucus gland hyperplasia, bronchial hyper-responsiveness, angiogenesis, collagen deposition and airway wall fibrosis (Pascual and Peters, 2005). Few data have been published on the potential role of MMP-12 in asthma or in allergic airway inflammation; however, MMP-12 mRNA levels have been shown to increase in the lungs of mice subjected to allergens exposure, as compared to sham exposed counterparts (Cataldo et al., 2002). MMP-12 deficient mice display less inflammation than wild type mice and show a less important peribronchial fibrosis when challenged repetitively for 3 months with allergens (Warner et al., 2004), clearly indicating that in these models MMP-12 plays an important proinflammatory role in the development of the allergic response. In another experimental model of allergic bronchial asthma it has been shown an elevated MMP-12 up-regulation after the induction of asthmatic reaction in rats (Chiba et al., 2007).

MMP-12 is mainly expressed in airway epithelia and alveolar macrophages under the control of TNF- $\alpha$, EGF and IFN- $\gamma$ (Lavigne et al., 2004). An up-regulation of MMP-12 was also observed in bronchial smooth muscle cells by IL-1 $\beta$ and TNF$\alpha$, contributing to the altered extracellular matrix composition and to the degradative environment in the airway during chronic asthma (Araujo et al., 2008).

8.1.3.4.3. Lung fibrosis. Lung fibrosis is characterized by an increase in the number of fibroblasts in the interalveolar septa together with an increase in collagen and elastin deposition. This thickening of the septa results in the loss of elasticity and decreased gas exchange and the most severe forms of lung fibrosis (such as idiopathic pulmonary fibrosis) are fatal. The administration of bleomycin in rodent airways induces similar inflammatory and fibrotic responses as those observed in patients with pulmonary fibrosis. During the acute phase of this bleomycin-induced injury, a significant activation of MMP-12 has been observed in mice and rats (Koslowski et al., 1998; Swiderski et al., 1998). It has also been shown that pro-MMP-12 is converted into its active form earlier after bleomycin treatment during the peak time of macrophage levels and is associated only to areas of hemorrhagy (Swaisgood et al., 2000). In an immune complex-induced acute lung injury model using mice containing a targeted disruption of the MMP-12 gene, neutrophil influx into the alveolar space and lung permeability in knockout mice has been reduced by $50 \%$ of that observed in wild-type littermates. These results have been correlated with the histological evidence of a reduced injury in MMP-12-knockout mice (Warner et al., 2001b).

Using inducible transgenic mouse models for IL-13 and/or TGF- $\beta$ it has been shown that MMP-12 deficiency regulates cytokine-induced inflammation and fibrosis; however, the mechanism remains unclear (Kang et al., 2007; Lanone et al., 2002). MMP-12-knockout mice were reported to be protected against Fas-L and bleomycin or transforming growth factor- $\beta$ (TGF- $\beta$ )-induced fibrosis (Kang et al., 2007; Matute-Bello et al., 2007), even though another study failed to detect any change in inflammation or collagen deposition in MMP-12-deficient mice in bleomycin-induced pulmonary fibrosis (Manoury et al., 2006).

Finally, it has been observed that IL-13-induced alveolar enlargement, lung enlargement, compliance alterations, and respiratory failure and death were markedly decreased in the absence of MMP-12. A deficiency in MMP-12 decreases the recovery of leukocytes, eosinophils, and macrophages, but not of lymphocytes or neutrophils (Lanone et al., 2002).

8.1.3.4.4. Idiopathic pneumonia syndrome (IPS). Idiopathic pneumonia syndrome (IPS) is a significant clinical problem encountered among patients treated with bone marrow transplantation (BMT). Although the specific etiology of IPS remains unclear, its mechanism includes cytokine-modulated immunological mechanisms during the acute and chronic phases after BMT leading to progressive, inflammatory and fibrotic lung disease. In recent findings it was shown that MMP-12 is important for limiting the development of IPS by allowing proper remodeling of extracellular matrix and effective repair of BMTrelated injury (England et al., 2011).

8.1.3.5. Diseases of the digestive system. Infection with the parasitic helminth Schistosoma mansoni brings about a significant liver fibrosis and extracellular matrix (ECM) remodeling. Global gene analysis reveal that MMP-12 is highly induced in the 
liver and lung in response to S. mansoni eggs (Sandler et al., 2003); therefore, a role of MMP-12 in the chronic helminth-induced inflammation and fibrosis has been postulated. In their study Satish and co-workers, using MMP-12-deficient mice, observe that MMP-12 promotes inflammation and Th2 cytokine-driven fibrosis in schistosomiasis by limiting the expression of ECM-degrading MMPs, such as MMP-2 and MMP-13 (Madala et al., 2010).

\subsection{MMP-19}

\subsubsection{Structure and activation}

MMP-19, also known as RASI-1, is a soluble 54 kDa protease with structural features that hinder its classification in each MMPs subfamily (see Fig. 1 and Table 2): the separated chromosomal localization (14q21) further reinforces this statement (Pendas et al., 1997).

Since the three-dimensional structure of the molecule is not available, some major structural insights have been deduced from its primary sequence and include: (i) an acidic residues-rich hinge region, (ii) a 26 residues-tail at the C-terminus hemopexin-like domain, where two putative $\mathrm{N}$-glycosylation sites are further located (iii) an additional cysteine residue in the catalytic site with no apparent functional role (Mueller et al., 2000a; Pendas et al., 1997; Stracke et al., 2000).

The catalytic zinc ion is coordinated by the conserved consensus sequence, but the domain structure significantly differ from that of other soluble MMPs and does not harbor any predictable glycosylation sites (Stracke et al., 2000). The hemopexin domain adopts the classical $\beta$-propeller conformation and includes several Thr residues essential for cell surface localization of the enzyme (Mysliwy et al., 2006).

\subsubsection{Biological features}

Promoter elements of MMP-19 gene suggest either a constitutive or inducible expression of the enzyme (Mueller et al., 2000a). As a matter of the fact, vascular smooth muscle cells, epithelial and basal keratinocytes in vitro constitutively secrete the enzyme, whereas endotheliocytes and macrophages do express it upon stimulation (Murphy et al., 1999). Similarly, the MMP-19 distribution in vivo is physiologically relevant in adult lung, intestine, pancreas, ovary and placenta, while circulating leukocytes and monocytes/macrophages do not secrete appreciable amount of this enzyme. Stimuli that trigger its expression have not been defined yet with the exception of TNF- $\alpha$ (Hieta et al., 2003).

Substrates of MMP-19 initially included collagen IV, tenascin C, fibrin, fibrinogen, laminin, nidogen envisaging, consistently with the constitutive basal expression, the association of MMP-19 biological activity to pro-homeostatic role in ECM turn-over and remodeling (Pendas et al., 1997) (see Table 3). The subsequent identification of novel substrates, as aggrecan, IGFBP-3 and plasminogen has revealed a broader contribute of MMP-9 to the aetiology of physiological and pathological processes (Brauer et al., 2011). Inhibition by natural inhibitors mainly occurs through interaction of the hemopexin domain with TIMP-2 and TIMP-4.

\subsubsection{Neoplastic diseases}

8.2.3.1. Epidemiological and molecular aspects. Association of MMP-19 with tumor aetiology is controversial as from divergent findings in animal models and in epidemiological studies. Only few aspects of its activity have been recently clarified (Brauer et al., 2011; Müller et al., 2010), and several aspects still remain obscure.

Elevated concentrations of MMP-19 are found in the microenvironment of some human tumors. In particular, tumors from the central nervous system, such as glioma, display a diffuse MMP-19 expression which further correlates with an advanced state and invasiveness of the tumor (Brauer et al., 2011; Chan et al., 2011; Velinov et al., 2007). Nonetheless, MMP-19 quantification has become a WHO-accepted value for human glioma stadiation (Lettau et al., 2010; Müller et al., 2010; Stojic et al., 2008).

MMP-19 expression further increases during melanoma progression being maximal in the advanced "vertical growth" phase and MMP-19-I- mice show normal development but decreased susceptibility to skin tumors. Notably, the enzyme up-regulation inversely correlates to the E-cadherin loss, suggesting the existence of a cell adhesiveness-related modulation of MMP-19 transcription which could account for the requisite of inducibility (Müller et al., 2010).

8.2.3.2. Invasiveness. The affinity for matrix and, mainly, basement membrane components inevitably links MMP-19 activity to cell invasiveness. In this respect, consistent evidences highlight a role in the modulation of cell motility even under physiological conditions: degradation of laminin is associated to promotion of epithelial cells as well as keratinocytes migration and proliferation (Sadowski et al., 2003, 2005). Myeloid cells and T-lymphocytes efficiently compartmentalize the enzyme at their leading edge through interaction with unknown cell surface molecules, to improving extravasation and migration toward the flogistic site (Beck et al., 2008; Mauch et al., 2002). Additional evidence of a positive influence of MMP-19 in tumor growth come from studies in vitro on breast cancer cells where siRNA-mediated inhibition of the enzyme down-regulates tumor invasiveness (Hegedüs et al., 2008).

However, the controversy about MMP-19 association to neoplastic diseases stems from several evidences that question the pro-malignant effect; thus, in the advanced grade human tumors MMP-19 is found down-regulated (Müller et al., 2010). Furthermore, in experimental models MMP-19 content in epithelial cells is detectable until the malignant transformation occurs (Impola et al., 2003). Similarly, mice lacking MMP-19 show increased angiogenesis and tumor malignancy (Jost et al., 2006). 
8.2.3.3. Angiogenesis. There is compelling evidence that angiogenesis is a critical step of tumor progression which could be targeted by MMP-19. The contribution of the enzyme to the promotion of physiological angiogenesis is documented; thus, MMP-19-mediated degradation of nidogen provides a scaffold for endothelial cells migration, furnishing stimuli for the dedifferentiation (Titz et al., 2004). Conversely, an anti-angiogenic role in tumors is actually more convincing, as from MMP-19 deficient mice which show early onset of tumoral angiogenesis (Jost et al., 2006). Intriguingly, a molecular rationale for this observation comes from plasminogen: fragments released upon MMP-19 degradation exhibit angiostatin-like properties and reduce endothelial cells proliferation and sprouting (Brauer et al., 2011).

\subsubsection{Non neoplastic diseases}

8.2.4.1. Diseases of the nervous system.

8.2.4.1.1. Multiple sclerosis. MMP-19 expression is highly up-regulated in multiple sclerosis lesions and decreases upon therapeutical administration of interferon- $\beta$ (Van Horssen et al., 2006).

Glial cells provide the reservoir of MMP-19 and, interestingly, surrounding-lesions astrocytes, which do not constitutively secrete the enzyme, induce the expression in response to not yet identified pro-inflammatory stimuli.

In MS lesions, aberrant degradation of Tenascin C occurs, leading to impaired neurite remyelinization. As previously reported, tenascin $\mathrm{C}$ is cleaved very efficiently by MMP-19.

MMP-19 further cleaves aggrecan, another molecule which undergoes proteolytical degradation in the onset and progression of MS. Therefore, MMP-19 pathogenic activity is presumably linked to destruction of axons architecture (Bernal et al., 2009).

8.2.4.2. Diseases of joints and muscular system. RASI-I, the alternative scientific name of the enzyme, is the acronym of Rheumatoid Arthritis Synovium Inflamed -1 because the enzyme has been initially found in the inflamed synovium of patients affected by this auto-immune disease (Sedlacek et al., 1998). Subsequent investigations about its role and biology have revealed the insights above discussed, but the association of MMP-19 and rheumatoid arthritis, even though devoid of molecular findings, has always represented an intriguing matter of discussion. In this sense, IgG Anti-MMP-19 antibodies have been identified in synovial fluid, allowing to hypothesize a direct involvement of the enzyme in the onset of auto-immune cascade (Sedlacek et al., 1998). Moreover, MMP-19 degrades the cartilage Oligomeric Matrix Protein (COMP) and aggrecan, compromising cartilage integrity (Stracke et al., 2000).

\subsection{MMP-21}

\subsubsection{Structure}

No details about the MMP-21 structure are available up to now. On the other hand, bio-informatic analysis of the aminoacidic sequence suggests a conserved domain composition, including the propeptide, the catalytic and the hemopexin-like domains (Marchenko et al., 2003).

\subsubsection{Biological Features}

The only available informations come from analysis of promoter elements which envisage a highly tissue-specific distribution of the enzyme. Transcription factors binding sites suggest that its expression should be modulated during differentiative processes and in embryogenesis (Ahokas et al., 2002, 2003; Skoog et al., 2009).

\subsubsection{Neoplastic diseases}

In several human tumors, including colorectal cancer, breast cancer, prostate cancer, pancreatic cancer, Merkel cell carcinoma, hepatocellular carcinoma, up-regulated levels of MMP-21 have been found, generally in association with poor prognosis (Bister et al., 2007; Hegedüs et al., 2008; Huang et al., 2011; Suomela et al., 2009; Wu et al., 2011b).

However, no molecular mechanisms of its pathological activity have been described.

\section{4. $M M P-28$}

\subsubsection{Structure and function}

Matrix metalloproteinase-28 (MMP-28), also known as epilysin, is a newly discovered MMP expressed in a number of normal tissues, such as testis, lung, intestine, heart brain and skin (Bernal et al., 2005; Illman et al., 2001; Lohi et al., 2001) as well as in a variety of tumors and tumor cell lines (Marchenko and Strongin, 2001).

MMP-28 is a $59 \mathrm{kDa}$ protein, closely related to MMP-19 at the level of the amino acid sequence (Mueller et al., 2000b), with a great similarity (97\%) in the catalytic domain between human and mouse proteins and overall $85 \%$ identity (Illman et al., 2008).

MMP-28 contains an activation sequence recognized by the furin endoprotease following the pro-domain (Mueller et al., 2000a,b), but it does not include domains characteristic of other furin-dependent MMP families (such as the disintegrin and thrombospondin-like regions) or the transmembrane group, as found in membrane-type MMPs (Lohi et al., 2001) (see Fig. 1). Moreover, the MMP-28 promoter has a unique conserved GF-box that is required for basal expression in keratinocytes (Illman et al., 2001). 
An overexpression of MMP-28 was observed in multiple disease states (Overall and Kleifeld, 2006b), but it was particularly evident in osteoarthritic cartilage, degenerated intervertebral disc (IVD) tissue and during conditions of demyelation (Davidson et al., 2006; Kevorkian et al., 2004; Werner et al., 2008).

Despite increasing interest in the role of MMP-28, little is known about its substrates (see Table 3). Recombinant MMP-28 has been reported to degrade casein in vitro and it is thought to cleave several neural proteins such as neurite outgrowth inhibitor A (Nogo-A), neural cell adhesion molecule (NCAM-1) and neuregulin 1 (NRG1) (Lohi et al., 2001; Werner et al., 2007, 2008).

\subsubsection{Neoplastic diseases}

Increased expression of MMP-28 was observed in a variety of tumors and tumor cell lines and its role seems to vary based on tumor type and stage of the disease (Lin et al., 2006; Marchenko and Strongin, 2001; Wallard et al., 2006). Only in the case of colon carcinoma MMP-28, unlike other MMPs, is downregulated, as compared to normal tissues (Bister et al., 2004).

Lin and colleagues have observed a closed correlation between MMP-28 levels and oral squamous cell carcinoma (OSCC), supporting a role for MMP-28 in the anchorage-independent growth of both OSCC and esophageal carcinomas (Lin et al., 2006).

Importantly, an overexpression of MMP-28 in lung adenocarcinoma cells induces an epithelial to mesenchymal transition (EMT) via activation of the latent transforming growth factor $\beta$ (TGF- $\beta$ ) (Illman et al., 2006; Illman et al., 2008). MMP-28-induced EMT is associated with loss of E-cadherin, a major mediator of cell-cell adhesion, as well as increased expression of MMP-9 (gelatinase B) and MMP-14 (MT1-MMP) (Illman et al., 2006; Illman et al., 2008).

Recent immuno-histochemical studies indicate a marked overexpression of MMP-28 in gastric carcinoma relative to normal epithelial cells (Jian et al., 2011). MMP-28 appears to be significantly associated with the depth of tumor invasion, lymph node metastasis and a poorer overall survival, representing a potential novel therapeutic target for the prevention and treatment of metastases in gastric cancer (Jian et al., 2011).

\subsubsection{Non-neoplastic diseases}

8.4.3.1. Disease of the nervous system. MMP-28 activity was shown to be involved in the development of several aspects of the neural microenvironment (Werner et al., 2007). MMP-28 plays an evolutionarily conserved role in neural development and it likely modulates the axonal-glial extracellular microenvironment (Werner et al., 2007). In addition, protein expression is inversely correlated with the expression of myelin-associated glycoprotein (MAG) during nerve regeneration (Werner et al., 2007).

Given the temporally regulated pattern of expression of MMP-28 prior to myelination in both developmental and regenerative states, it is likely that MMP-28 plays a functional role in the maturation of nerves. Moreover, under conditions of demyelination in vivo, MMP-28 is upregulated, inducing signaling in vitro consistent with myelination inhibition and suggesting that inhibition of MMP-28 may be beneficial for promoting the myelin repair (Werner et al., 2008).

8.4.3.2. Disease of joints and muscular system. The regulation and elevation in expression of the catabolic MMPs is of high importance in the human intervertebral disc (IVD), since it results in matrix destruction associated with disc degeneration. A role of MMP-28 in ECM modulation in the healthy and degenerating disc has been suggested (Gruber et al., 2009), reporting that both MMP-28 and the MMP-28 precursor are detectable in the disc cell cytoplasm and in the ECM of more degenerated specimens, with greater cellular localization in the outer annulus and in herniated disc specimens (Gruber et al., 2009). Recently, the expression levels of MMP-28 have been investigated in traumatic and degenerative human intervertebral disc (IVD) tissue with different degrees of degeneration (Klawitter et al., 2011). The findings demonstrate that while MMP-28 expression increases in individual cases with trauma or disc degeneration, there is no significant correlation between the grade of disease and MMP-28 expression. In addition, unlike many other MMPs, MMP-28 is not regulated by various inflammatory mediators (IL-1 $\beta$, TNF- $\alpha$, LPS) or by the histone deacetylase inhibitor trichostatin A (Klawitter et al., 2011). Future studies are needed to identify the role of MMP-28 in the intervertebral disc more conclusively.

8.4.3.3. Wound healing. MMP-28 is expressed in the basal and suprabasal epidermis in intact skin, whilst in the injured skin its expression is observed in basal keratinocytes both at the wound edge and some distance from it (Lohi et al., 2001; Saarialho-Kere et al., 2002). Wound healing studies showed that MMP-28 was spatially and temporally regulated, with a strong up-regulation of MMP-28 occurring in mitotic cells of wounded skin; this suggests that MMP-28 may be required to restructure the basement membrane or to degrade adhesive proteins between keratinocytes in order to supply new cells for the migrating front (Lohi et al., 2001; Saarialho-Kere et al., 2002). In primary cultures of human keratinocytes, MMP-28 expression is strongly stimulated by tumor necrosis factor $\alpha$ (TNF- $\alpha$ ) (Saarialho-Kere et al., 2002). Moreover, mechanical compression significantly up-regulates MMP-28 secretion in hypertrophic scars (Reno et al., 2005).

In a recent work, the localization of both catalytically active and mutated inactive, overexpressed MMP-28 has been characterized in established epithelial cell lines (Heiskanen et al., 2009). These authors found that the protein localizes abundantly to the basolateral side of the cells and associates with a drastic reduction of basolateral ECM, as observed by the disappearance of collagen type IV, laminin and fibronectin. 


\section{Acknowledgements}

The authors are very thankful to Profs. Paolo Ascenzi, Hideaki Nagase, Ghislain Opdenakker, Chris M. Overall, Tayebeh Pourmotabbed, James P. Quigley, Harald Tschesche and Philippe van den Steen for several fruitful discussions during the writing of this review.

\section{References}

Adachi, Y., Yamamoto, H., Itoh, F., Arimura, Y., Nishi, M., Endo, T., Imai, K., 2001. Clinicopathologic and prognostic significance of matrilysin expression at the invasive front in human colorectal cancers. Int. J. Cancer 95, 290-294.

Adachi, Y., Yamamoto, H., Itoh, F., Hinoda, Y., Okada, Y., Imai, K., 1999. Contribution of matrilysin (MMP-7) to the metastatic pathway of human colorectal cancers. Gut 45, 252-258.

Agarwal, A.K., Fryns, J.P., Auchus, R.J., Garg, A., 2003a. Zinc metalloproteinase, ZMPSTE24, is mutated in mandibuloacral dysplasia. Hum. Mol. Genet. 12, 1995-2001.

Agarwal, D., Goodison, S., Nicholson, B., Tarin, D., Urquidi, V., 2003b. Expression of matrix metalloproteinase 8 (MMP-8) and tyrosinase-related protein-1 (TYRP-1) correlates with the absence of metastasis in an isogenic human breast cancer model. Differentiation 71, 114-125.

Agrawal, S.M., Silva, C., Tourtellotte, W.W., Yong, V.W., 2011. EMMPRIN: a novel regulator of leukocyte transmigration into the CNS in multiple sclerosis and experimental autoimmune encephalomyelitis. J. Neurosci. 31, 669-677.

Ahmad, Z., Zackai, E., Medne, L., Garg, A., 2010. Early onset mandibuloacral dysplasia due to compound heterozygous mutations in ZMPSTE24. Am. J. Med. Genet. 152A, 2703-2710.

Ahmed, S., Pakozdi, A., Koch, A.E., 2006. Regulation of interleukin-1beta-induced chemokine production and matrix metalloproteinase 2 activation by epigallocatechin-3-gallate in rheumatoid arthritis synovial fibroblasts. Arthritis Rheum. 54, 2393-2401.

Ahn, G.O., Brown, J.M., 2008. Matrix metalloproteinase-9 is required for tumor vasculogenesis but not for angiogenesis: role of bone marrow-derived myelomonocytic cells. Cancer Cell 13, 193-205.

Ahokas, K., Lohi, J., Illman, S.A., Llano, E., Elomaa, O., Impola, U., Karjalainen-Lindsberg, M.L., Saarialho-Kere, U., 2003. Matrix metalloproteinase-21 is expressed epithelially during development and in cancer and is up-regulated by transforming growth factor-beta1 in keratinocytes. Lab. Invest. 83, $1887-1899$

Ahokas, K., Lohi, J., Lohi, H., Elomaa, O., Karjalainen-Lindsberg, M.L., Kere, J., Saarialho-Kere, U., 2002. Matrix metalloproteinase-21, the human orthologue for XMMP, is expressed during fetal development and in cancer. Gene 301, 31-41.

Aihara, R., Mochiki, E., Nakabayashi, T., Akazawa, K., Asao, T., Kuwano, H., 2005. Clinical significance of mucin phenotype, beta-catenin and matrix metalloproteinase 7 in early undifferentiated gastric carcinoma. Br. J. Surg. 92, 454-462.

Airola, K., Johansson, N., Kariniemi, A.L., Kähäri, V.M., Saarialho-Kere, U., 1997. Human collagenase-3 is expressed in malignant squamous epithelium of the skin. J. Invest. Dermatol. 109, 225-331.

Akhtar, N., Rasheed, Z., Ramamurthy, S., Anbazhagan, A.N., Voss, F.R., Haqqi, T.M., 2010. MicroRNA-27b regulates the expression of matrix metalloproteinase 13 in human osteoarthritis chondrocytes. Arthritis Rheum. 62, 1361-1371.

Ala-aho, R., Grenman, R., Seth, P., Kähäri, V.M., 2002a. Adenoviral delivery of p53 gene suppresses expression of collagenase-3 (MMP-13) in squamous carcinoma cells. Oncogene 21, 1187-1195.

Ala-Aho, R., Johansson, N., Baker, A.H., Kähäri, V.M., 2002b. Expression of collagenase-3 (MMP-13) enhances invasion of human fibrosarcoma HT-1080 cells. Int. J. Cancer 97, 283-289.

Alam, M., Mohammad, A., Rahman, S., Todd, K., Shuaibm, A., 2011. Hyperthermia up-regulates matrix metalloproteinases and accelerates basement membrane degradation in experimental stroke. Neurosci. Lett. 495, 135-149.

Albrecht, M., Doroszewicz, J., Gillen, S., Gomes, I., Wilhelm, B., Stief, T., Aumüller, G., 2004. Proliferation of prostate cancer cells and activity of neutral endopeptidase is regulated by bombesin and IL-1beta with IL-1beta acting as a modulator of cellular differentiation. Prostate 58, 82-94.

Amberger, V.R., Paganetti, P.A., Seulberger, H., Eldering, J.A., Schwab, M.E., 1994. Characterization of a membrane-bound metalloendoprotease of rat C6 glioblastoma cells. Cancer Res. 54, 4017-4025.

Annabi, B., Shedid, D., Ghosn, P., Kenigsberg, R.L., Desrosiers, R.R., Bojanovski, M.W., Beaulieu, E., Nassif, E., Moumdjan, R., Beliveau, R., 2002. Differential regulation of matrix metalloproteinase activities in abdominal aortic aneurysms. J. Vasc. Surg. 35, 539-546.

Anthony, D.C., Miller, K.M., Fearn, S., Townsend, M.J., Opdenakker, G., Wells, G.M., Clements, J.M., Chandler, S., Gearing, A.J., Perry, V.H., 1998. Matrix metalloproteinase expression in an experimentally-induced DTH model of multiple sclerosis in the rat CNS. J. Neuroimmunol. 87, 62-72.

Apte, S.S., 2009. A disintegrin-like and metalloprotease (reprolysin type) with thrombospondin type 1 motifs: the ADAMTS family. Int. J. Biochem. Cell Biol. $36,981-985$

Araujo, B.B., Dolhnikoff, M., Silva, L.F., Elliot, J., Lindeman, J.H., Ferreira, D.S., Mulder, A., Gomes, H.A., Fernezlian, S.M., James, A., Mauad, T., 2008. Extracellular matrix components and regulators in the airway smooth muscle in asthma. Eur. Respir. J. 32, 61-69.

Ardi, V.C., Kupriyanova, T.A., Deryugina, E.I., Quigley, J.P., 2007. Human neutrophils uniquely release TIMP-free MMP-9 to provide a potent catalytic stimulator of angiogenesis. Proc. Natl. Acad. Sci. USA 104, 20262-20267.

Aresu, L., Benali, S., Garbisa, S., Gallo, E., Castagnaro, M., 2011. Matrix metalloproteinases and their role in the renal epithelial mesenchymal transition. Histol. Histopathol. 26, 307-313

Arlt, M., Kopitz, C., Pennington, C., Watson, K.L., Krell, H.W., Bode, W., Gansbacher, B., Khokha, R., Edwards, D.R., Kruger, A., 2002. Increase in gelatinasespecificity of matrix metalloproteinase inhibitors correlates with antimetastatic efficacy in a T-cell lymphoma model. Cancer Res. 62, 5543-5550.

Arthur, M.J., 2000. Fibrogenesis II. Metalloproteinases and their inhibitors in liver fibrosis. Am. J. Physiol. Gastrointest. Liver Physiol. 279, G245-G249.

Asahina, M., Yoshiyama, Y., Hattori, T., 2001. Expression of matrix metalloproteinase-9 and urinary-type plasminogen activator in Alzheimer's disease brain Clin. Neuropathol. 20, 60-63.

Atkinson, J.J., Senior, R.M., 2003. Matrix metalloproteinase-9 in lung remodeling. Am. J. Respir. Cell Mol. Biol. 28, 12-24.

Atkinson, J., Holmbeck, K., Yamada, S., Birkedal-Hansen, H., Parks, W.C., Senior, R.M., 2005. Membrane-type 1 matrix metalloproteinase is required for normal alveolar development. Dev. Dyn. 232, 1079-1090.

Atkinson, J.J., Toennies, H.M., Holmbeck, K., Senior, R.M., 2007. Membrane type 1 matrix metalloproteinase is necessary for distal airway epithelial repair and keratinocyte growth factor receptor expression after acute injury. Am. J. Physiol. Lung Cell. Mol. Physiol. 293, L600-L610.

Aureli, L., Gioia, M., Cerbara, I., Monaco, S., Fasciglione, G.F., Marini, S., Ascenzi, P., Topai, A., Coletta, M., 2008. Structural bases for substrate and inhibitor recognition by matrix metalloproteinases. Curr. Med. Chem. 15, 2192-2222.

Avolio, C., Ruggieri, M., Giuliani, F., Liuzzi, G.M., Leante, R., Riccio, P., Livrea, P., Trojano, M., 2003. Serum MMP-2 and MMP-9 are elevated in different multiple sclerosis subtypes. J. Neuroimmunol. 136, 46-53.

Ayoub, A.E., Cai, T.Q., Kaplan, R.A., Luo, J., 2005. Developmental expression of matrix metalloproteinases 2 and 9 and their potential role in the histogenesis of the cerebellar cortex. J. Comp. Neurol. 481, 403-415.

Azar, D.T., Casanova, F.H., Mimura, T., Jain, S., Zhou, Z., Han, K.Y., Chang, J.H., 2010. Corneal epithelial MT1-MMP inhibits vascular endothelial cell proliferation and migration. Cornea 29, 321-330.

Babusyte, A., Stravinskaite, K., Jeroch, J., Lotvall, J., Sakalauskas, R., Sitkauskiene, B., 2007. Patterns of airway inflammation and MMP-12 expression in smokers and ex-smokers with COPD. Respir. Res. 8, 81 
Baciu, P.C., Suleiman, E.A., Deryugina, E.I., Strongin, A.Y., 2003. Membrane type-1 matrix metalloproteinase (MT1-MMP) processing of pro-alphav integrin regulates cross-talk between alphavbeta3 and alpha2beta1 integrins in breast carcinoma cells. Exp. Cell Res. 291, $167-175$.

Badiga, A.V., Chetty, C., Kesanakurti, D., Are, D., Gujrati, M., Klopfenstein, J.D., Dinh, D.H., Rao, J.S., 2011. MMP-2 siRNA inhibits radiation-enhanced invasiveness in glioma cells. PLoS One 6, e20614.

Bafetti, L.M., Young, T.N., Itoh, Y., Stack, M.S., 1998. Intact vitronectin induces matrix metalloproteinase-2 and tissue inhibitor of metalloproteinases-2 expression and enhanced cellular invasion by melanoma cells. J. Biol. Chem. 273, 143-149.

Baines, K.J., Wood, L.G., Gibson, P.G., 2009. The nutrigenomics of asthma: molecular mechanisms of airway neutrophilia following dietary antioxidant withdrawal. OMICS 13, 355-365.

Balbin, M., Fueyo, A., Knäuper, V., Pendas, A.M., Lopez, J.M., Jimenez, M.G., Murphy, G., Lopez-Otin, C., 1998. Collagenase 2 (MMP-8) expression in murine tissue-remodeling processes. Analysis of its potential role in postpartum involution of the uterus. J. Biol. Chem. 273, 23959-23968.

Balbin, M., Fueyo, A., Knäuper, V., Lopez, J.M., Alvarez, J., Sanchez, L.M., Quesada, V., Bordallo, J., Murphy, G., Lopez-Otin, C., 2001. Identification and enzymatic characterization of two diverging murine counterparts of human interstitial collagenase (MMP-1) expressed at sites of embryo implantation. J. Biol. Chem. 276, 10253-10262.

Balbin, M., Fueyo, A., Tester, A.M., Pendas, A.M., Pitiot, A.S., Astudillo, A., Overall, C.M., Shapiro, S.D., Lopez-Otin, C., 2003. Loss of collagenase-2 confers increased skin tumor susceptibility to male mice. Nat. Genet. 35, 252-257.

Banda, M.J., Rice, A.G., Griffin, G.L., Senior, R.M., 1988. Alpha 1-proteinase inhibitor is a neutrophil chemoattractant after proteolytic inactivation by macrophage elastase. J. Biol. Chem. 263, 4481-4484.

Baramova, E.N., Bajou, K., Remacle, A., L'Hoir, C., Krell, H.W., Weidle, U.H., Noel, A., Foidart, J.M., 1997. Involvement of PA/plasmin system in the processing of pro-MMP-9 and in the second step of pro-MMP-2 activation. FEBS Lett. 405, 157-162.

Barascuk, N., Vassiliadis, E., Larsen, L., Wang, J., Zheng, Q., Xing, R., Cao, Y., Crespo, C., Lapret, I., Sabatini, M., Villeneuve, N., Vilaine, J.P., Rasmussen, L.M., Register, T.C., Karsdal, M.A., 2011. Development and validation of an enzyme-linked immunosorbent assay for the quantification of a specific MMP-9 mediated degradation fragment of type III collagen - a novel biomarker of atherosclerotic plaque remodeling. Clin. Biochem. $44,900-906$.

Barksby, H.E., Milner, J.M., Patterson, A.M., Peake, N.J., Hui, W., Robson, T., Lakey, R., Middleton, J., Cawston, T.E., Richards, C.D., Rowan, A.D., 2006. Matrix metalloproteinase 10 promotion of collagenolysis via procollagenase activation: implications for cartilage degradation in arthritis. Arthritis Rheum. 54 , $3244-3253$.

Barmina, O.Y., Walling, H.W., Fiacco, G.J., Freije, J.M., Lopez-Otin, C., Jeffrey, J.J., Partridge, N.C., 1999. Collagenase-3 binds to a specific receptor and requires the low-density lipoprotein receptor-related protein for internalization. J. Biol. Chem. 274, 30087-30093.

Barnes, P.J., Shapiro, S.D., Pauwels, R.A., 2003. Chronic obstructive pulmonary disease: molecular and cellular mechanisms. Eur. Respir. J. 22, 672-688.

Bar-Or, A., Nuttall, R.K., Duddy, M., Alter, A., Kim, H.J., Ifergan, I., Pennington, C.J., Bourgoin, P., Edwards, D.R., Yong, V.W., 2003. Analyses of all matrix metalloproteinase members in leukocytes emphasize monocytes as major inflammatory mediators in multiple sclerosis. Brain 126, $2738-2749$.

Barret, A.J., Rawlings, N.D., Woessner, J.F., 2004. The Handbook of Proteolytic Enzymes, second ed. Academic Press, San Diego, USA.

Barrowman, J., Michaelis, S., 2009. ZMPSTE24, an integral membrane zinc metalloprotease with a connection to progeroid disorders. Biol. Chem. 390, 761773.

Basset, P., Bellocq, J.P., Wolf, C., Stoll, I., Hutin, P., Limacher, J.M., Podhajcer, O.L., Chenard, M.P., Rio, M.C., Chambon, P., 1990. A novel metalloproteinase gene specifically expressed in stromal cells of breast carcinomas. Nature 348, 699-704.

Basset, P., Bellocq, J.P., Lefebvre, O., Noel, A., Chenard, M.P., Wolf, C., Anglard, P., Rio, M.C., 1997. Stromelysin-3: a paradigm for stroma-derived factors implicated in carcinoma progression. Crit. Rev. Oncol. Hematol. 26, 43-53.

Bassi, D.E., Mahloogi, H., Klein-Szanto, A.J., 2000. The proprotein convertases furin and PACE4 play a significant role in tumor progression. Mol. Carcinog. 28, 63-69.

Bau, B., Gebhard, P.M., Haag, J., Knorr, T., Bartnik, E., Aigner, T., 2002. Relative messenger RNA expression profiling of collagenases and aggrecanases in human articular chondrocytes in vivo and in vitro. Arthritis Rheum. 46, 2648-2657.

Bayes-Genis, A., Conover, C.A., Overgaard, M.T., Bailey, K.R., Christiansen, M., Holmes Jr., D.R., Virmani, R., Oxvig, C., Schwartz, R.S., 2001. Pregnancyassociated plasma protein A as a marker of acute coronary syndromes. N. Engl. J. Med. 345, 1022-1029.

Beck, I.M., Rückert, R., Brandt, K., Mueller, M.S., Sadowski, T., Brauer, R., Schirmacher, P., Mentlein, R., Sedlacek, R., 2008. MMP19 is essential for T cell development and T cell-mediated cutaneous immune responses. PLoS One 3, e2343.

Becker, J.W., Marcy, A.I., Rokosz, L.L., Axel, M.G., Burbaum, J.J., Fitzgerald, P.M., Cameron, P.M., Esser, C.K., Hagmann, W.K., Hermes, J.D., Springer, J.P., 1995. Stromelysin-1: three-dimensional structure of the inhibited catalytic domain and of the C-truncated proenzyme. Protein Sci. 4, $1966-1976$.

Belaaouaj, A., Shipley, J.M., Kobayashi, D.K., Zimonjic, D.B., Popescu, N., Silverman, G.A., Shapiro, S.D., 1995. Human macrophage metalloelastase. Genomic organization, chromosomal location, gene linkage, and tissue-specific expression. J. Biol. Chem. 270, 14568-14575.

Belaaouaj, A.A., Li, A., Wun, T.C., Welgus, H.G., Shapiro, S.D., 2000. Matrix metalloproteinases cleave tissue factor pathway inhibitor. Effects on coagulation. J. Biol. Chem. 275, 27123-27128.

Beliën, A.T., Paganetti, P.A., Schwab, M.E., 1999. Membrane-type 1 matrix metalloprotease (MT1-MMP) enables invasive migration of glioma cells in central nervous system white matter. J. Cell Biol. 144, 373-384.

Beliveau, A., Mott, J.D., Lo, A., Chen, E.I., Koller, A.A., Yaswen, P., Muschler, J., Bissell, M.J., 2010. Raf-induced MMP9 disrupts tissue architecture of human breast cells in three-dimensional culture and is necessary for tumor growth in vivo. Genes Dev. 24, 2800-2811.

Belkin, A.M., Akimov, S.S., Zaritskaya, L.S., Ratnikov, B.I., Deryugina, E.I., Strongin, A.Y., 2001. Matrix-dependent proteolysis of surface transglutaminase by membrane-type metalloproteinase regulates cancer cell adhesion and locomotion. J. Biol. Chem. 276, 18415-18422.

Bendardaf, R., Lamlum, H., Vihinen, P., Ristamaki, R., Laine, J., Pyrhonen, S., 2003. Low collagenase-1 (MMP-1) and MT1-MMP expression levels are favourable survival markers in advanced colorectal carcinoma. Oncology 65, 337-346.

Bendrik, C., Robertson, J., Gauldie, J., Dabrosin, C., 2008. Gene transfer of matrix metalloproteinase-9 induces tumor regression of breast cancer in vivo. Cancer Res. 68, 3405-3412.

Benesova, Y., Vasku, A., Stourac, P., Hladikova, M., Beranek, M., Kadanka, Z., Novotna, H., Bednarik, J., 2008. Matrix metalloproteinase-9 and matrix metalloproteinase-2 gene polymorphisms in multiple sclerosis. J. Neuroimmunol. 205, 105-109.

Benesova, Y., Vasku, A., Novotna, H., Litzman, J., Stourac, P., Beranek, M., Kadanka, Z., Bednarik, J., 2009. Matrix metalloproteinase-9 and matrix metalloproteinase-2 as biomarkers of various courses in multiple sclerosis. Mult. Scler. 15, 316-322.

Bergers, G., Brekken, R., McMahon, G., Vu, T.H., Itoh, T., Tamaki, K., Tanzawa, K., Thorpe, P., Itohara, S., Werb, Z., Hanahan, D., 2000. Matrix metalloproteinase9 triggers the angiogenic switch during carcinogenesis. Nat. Cell Biol. 2, 737-744.

Bergmann, U., Michaelis, J., Oberhoff, R., Knäuper, V., Beckmann, R., Tschesche, H., 1998. Enzyme linked immunosorbent assays (ELISA) for the quantitative determination of human leukocyte collagenase and gelatinase. J. Clin. Chem. Clin. Biochem. 27, 351-359.

Bernal, F., Hartung, H.P., Kieseier, B.C., 2005. Tissue mRNA expression in rat of newly described matrix metalloproteinases. Biol. Res. 38, 267-271.

Bernal, F., Elias, B., Hartung, H.P., Kieseier, B.C., 2009. Regulation of matrix metalloproteinases and their inhibitors by interferon-beta: a longitudinal study in multiple sclerosis patients. Mult. Scler. 15, 721-727.

Bernstein, H.G., Bukowska, A., Krell, D., Bogerts, B., Ansorge, S., Lendeckel, U., 2003. Comparative localization of ADAMs 10 and 15 in human cerebral cortex normal aging, Alzheimer disease and Down syndrome. J. Neurocytol. 32, 153-160.

Berry, D.L., Rose, C.S., Remo, B.F., Brown, D.D., 1998. The expression pattern of thyroid hormone response genes in remodeling tadpole tissues defines distinct growth and resorption gene expression programs. Dev. Biol. 203, 24-35.

Betsuyaku, T., Nishimura, M., Takeyabu, K., Tanino, M., Venge, P., Xu, S., Kawakami, Y., 1999. Neutrophil granule proteins in bronchoalveolar lavage fluid from subjects with subclinical emphysema. Am. J. Respir. Crit. Care Med. 159, 1985-1991. 
Bhaskar, V., Yin, J., Mirza, A.M., Phan, D., Vanegas, S., Issafras, H., Michelson, K., Hunter, J.J., Kantak, S.S., 2011. Monoclonal antibodies targeting IL-1 beta reduce biomarkers of atherosclerosis in vitro and inhibit atherosclerotic plaque formation in apolipoprotein E-deficient mice. Atherosclerosis 216 , 313320.

Bhaskaran, R., Palmier, M.O., Lauer-Fields, J.L., Fields, G.B., Van Doren, S.R., 2008. MMP-12 catalytic domain recognizes triple helical peptide models of collagen V with exosites and high activity. J. Biol. Chem. 283, 21779-21788.

Billinghurst, R.C., Dahlberg, L., Ionescu, M., Reiner, A., Bourne, R., Rorabeck, C., Mitchell, P., Hambor, J., Diekmann, O., Tschesche, H., Chen, J., van Wart, H., Poole, A.R., 1997. Enhanced cleavage of type II collagen by collagenases in osteoarthritic articular cartilage. J. Clin. Invest. 99, $1534-1545$.

Bilousova, T.V., Rusakov, D.A., Ethell, D.W., Ethell, I.M., 2006. Matrix metalloproteinase-7 disrupts dendritic spines in hippocampal neurons through NMDA receptor activation. J. Neurochem. 97, 44-56.

Binder, C., Hagemann, T., Husen, B., Schulz, M., Einspanier, A., 2002. Relaxin enhances in vitro invasiveness of breast cancer cell lines by upregulation of matrix metalloproteases. Mol. Hum. Reprod. 8, 789-796.

Binker, M.G., Binker-Cosen, A.A., Gaisano, H.Y., de Cosen, R.H., Cosen-Binker, L.I., 2011. TGF- $\beta 1$ increases invasiveness of SW1990 cells through Rac1/ROS/NF$\kappa \mathrm{B} / \mathrm{IL}-6 / \mathrm{MMP}-2$. Biochem. Biophys. Res. Commun. 405, 140-145.

Bisson, C., Blacher, S., Polette, M., Blanc, J.F., Kebers, F., Desreux, J., Tetu, B., Rosenbaum, J., Foidart, J.M., Birembaut, P., Noel, A., 2003. Restricted expression of membrane type 1-matrix metalloproteinase by myofibroblasts adjacent to human breast cancer cells. Int. J. Cancer $105,7-13$

Bister, V.O., Salmela, M.T., Karjalainen-Lindsberg, M.L., Uria, J., Lohi, J., Puolakkainen, P., Lopez-Otin, C., Saarialho-Kere, U., 2004. Differential expression of three matrix metalloproteinases, MMP-19, MMP-26, and MMP-28, in normal and inflamed intestine and colon cancer. Dig. Dis. Sci. 49, 653-661.

Bister, V., Skoog, T., Virolainen, S., Kiviluoto, T., Puolakkainen, P., Saarialho-Kere, U., 2007. Increased expression of matrix metalloproteinases-21 and -26 and TIMP-4 in pancreatic adenocarcinoma. Mod. Pathol. 20, 1128-1140.

Biswas, M.H., Du, C., Zhang, C., Straubhaar, J., Languino, L.R., Balaji, K.C., 2010. Protein kinase D1 inhibits cell proliferation through matrix metalloproteinase2 and matrix metalloproteinase-9 secretion in prostate cancer. Cancer Res. 70, 2095-2104.

Björklund, M., Koivunen, E., 2005. Gelatinase-mediated migration and invasion of cancer cells. Biochim. Biophys. Acta 1755, 37-69.

Bjørn, S.F., Hastrup, N., Larsen, J.F., Lund, L.R., Pyke, C., 2000. Messenger RNA for membrane-type 2 matrix metalloproteinase, MT2-MMP, is expressed in human placenta of first trimester. Placenta 21, 170-176.

Bland, N.D., Pinney, J.W., Thomas, J.E., Turner, A.J., Isaac, R.E., 2008. Bioinformatic analysis of the neprilysin (M13) family of peptidases reveals complex evolutionary and functional relationships. BMC Evol. Biol. 23, 8-16.

Blavier, L., Lazaryev, A., Shi, X.H., Dorey, F.J., Shackleford, G.M., DeClerck, Y.A., 2010. Stromelysin-1 (MMP-3) is a target and a regulator of Wnt1-induced epithelial-mesenchymal transition (EMT). Cancer Biol. Ther. 10, 198-208.

Blobel, C.P., 2005. ADAMs: key components in EGFR signalling and development. Nat. Rev. Mol. Cell Biol. 6, 32-43.

Blumenthal, M.N., Zhong, W., Miller, M., Wendt, C., Connett, J.E., Pei, D., 2010. Serum metalloproteinase leukolysin (MMP-25/MT-6): a potential metabolic marker for atopy-associated inflammation. Clin. Exp. Allergy 40, 859-866.

Bode, W., 1995. A helping hand for collagenases: the haemopexin-like domain. Structure 3, 527-530.

Bode, W., Fernandez-Catalan, C., Tschesche, H., Grams, F., Nagasa, H., Maskos, K., 1999. Structural properties of matrix metalloproteinases. Cell. Mol. Life Sci. $55,639-652$.

Boldt, H.B., Conover, C.A., 2007. Pregnancy-associated plasma protein-A (PAPP-A): a local regulator of IGF bioavailability through cleavage of IGFBPs. Growth Horm. IGF Res. 17, 10-18.

Bolignano, D., Donato, V., Lacquaniti, A., Fazio, M.R., Bono, C., Coppolino, G., Buemi, M., 2010. Neutrophil gelatinase-associated lipocalin (NGAL) in human neoplasias: a new protein enters the scene. Cancer Lett. 288, 10-16.

Bolon, I., Devouassoux, M., Robert, C., Moro, D., Brambilla, C., Brambilla, E., 1997. Expression of urokinase-type plasminogen activator, stromelysin 1, stromelysin 3, and matrilysin genes in lung carcinomas. Am. J. Pathol. 150, 1619-1629.

Bonacci, G., Schopfer, F.J., Batthyany, C.I., Rudolph, T.K., Rudolph, V., Khoo, N.K., Kelley, E.E., Freeman, B.A., 2011. Electrophilic fatty acids regulate matrix metalloproteinase activity and expression. J. Biol. Chem. 286, 16074-16081.

Bord, S., Horner, A., Hembry, R.M., Compston, J.E., 1998. Stromelysin-1 (MMP-3) and stromelysin-2 (MMP-10) expression in developing human bone: potential roles in skeletal development. Bone 23, 7-12.

Borges, L.F., Touat, Z., Leclercq, A., Zen, A.A., Jondeau, G., Franc, B., Philippe, M., Meilhac, O., Gutierrez, P.S., Michel, J.B., 2009. Tissue diffusion and retention of metalloproteinases in ascending aortic aneurysms and dissections. Hum. Pathol. 40, 306-313.

Borkakoti, N., 2000. Structural studies of matrix metalloproteinases. J. Mol. Med. 78, 261-268.

Borrell-Pages, M., Rojo, F., Albanell, J., Baselga, J., Arribas, J., 2003. TACE is required for the activation of the EGFR by TGF-alpha in tumors. EMBO J. 22, 11141124.

Boulay, A., Masson, R., Chenard, M.P., El Fahime, M., Cassard, L., Bellocq, J.P., Sautes-Fridman, C., Basset, P., Rio, M.C., 2001. High cancer cell death in syngeneic tumors developed in host mice deficient for the stromelysin-3 matrix metalloproteinase. Cancer Res. 61, $2189-2193$.

Bourboulia, D., Stetler-Stevenson, W.G., 2010. Matrix metalloproteinases (MMPs) and tissue inhibitors of metalloproteinases (TIMPs): positive and negative regulators in tumor cell adhesion. Semin. Cancer Biol. 20, 161-168.

Bourguignon, L.Y., Gunja-Smith, Z., Iida, N., Zhu, H.B., Young, L.J., Muller, W.J., Cardiff, R.D., 1998. CD44v(3,8-10) is involved in cytoskeleton-mediated tumor cell migration and matrix metalloproteinase (MMP-9) association in metastatic breast cancer cells. J. Cell. Physiol. 176, $206-215$.

Bozzi, M., Inzitari, R., Sbardella, D., Monaco, S., Pavoni, E., Gioia, M., Marini, S., Morlacchi, S., Sciandra, F., Castagnola, M., Giardina, B., Brancaccio, A., Coletta, M., 2009. Enzymatic processing of beta-dystroglycan recombinant ectodomain by MMP-9: identification of the main cleavage site. IUBMB Life 61, 11431152 .

Bradbury, P.A., Zhai, R., Hopkins, J., Kulke, M.H., Heist, R.S., Singh, S., Zhou, W., Ma, C., Xu, W., Asomaning, K., Ter-Minassian, M., Wang, Z., Su, L., Christiani, D.C., Liu, G., 2009. Matrix metalloproteinase 1, 3 and 12 polymorphisms and esophageal adenocarcinoma risk and prognosis. Carcinogenesis 30, 793798.

Bralet, J., Schwartz, J.C., 2001. Vasopeptidase inhibitors: an emerging class of cardiovascular drugs. Trends Pharmacol. Sci. 22, 106-109.

Brauer, R., Beck, I.M., Roderfeld, M., Roeb, E., Sedlacek, R., 2011. Matrix metalloproteinase-19 inhibits growth of endothelial cells by generating angiostatinlike fragments from plasminogen. BMC Biochem. 12, 38.

Brew, K., Nagase, H., 2010. The tissue inhibitors of metalloproteinases (TIMPs): an ancient family with structural and functional diversity. Biochim. Biophys. Acta 1803, 55-71.

Briknarova, K., Grishaev, A., Banyai, L., Tordai, H., Patthy, L., Llinas, M., 1999. The second type II module from human matrix metalloproteinase 2: structure, function and dynamics. Structure 7, 1235-1245.

Brinckerhoff, C.E., Suzuki, K., Mitchell, T.I., 1990. Rabbit procollagenase synthesized and secreted by a high yield mammalian expression vector requires stromelysin (matrix metalloproteinase-3) for maximal activation. J. Biol. Chem. 265, 22262-22269.

Brinckerhoff, C.E., Rutter, J.L., Benbow, U., 2000. Interstitial collagenases as markers of tumor progression. Clin. Cancer Res. 6, 4823-4830.

Brinckerhoff, C.E., Matrisian, L.M., 2002. Matrix metalloproteinases: a tail of a frog that became a prince. Nat. Rev. Mol. Cell Biol. 3, $207-214$.

Broide, D.H., 2008. Immunologic and inflammatory mechanisms that drive asthma progression to remodelling. J. Allergy Clin. Immunol. 121, 560-570.

Brooks, P.C., Strömblad, S., Sanders, L.C., von Schalscha, T.L., Aimes, R.T., Stetler-Stevenson, W.G., Quigley, J.P., Cheresh, D.A., 1996. Localization of matrix metalloproteinase MMP-2 to the surface of invasive cells by interaction with integrin alpha v beta 3. Cell 85, $683-693$.

Brown, G.D., Nazarali, A.J., 2010. Matrix metalloproteinase-25 has a functional role in mouse secondary palate development and is a downstream target of TGF- $\beta 3$. BMC Dev. Biol. 10, 93.

Buhler, L.A., Samara, R., Guzman, E., Wilson, C.L., Krizanac-Bengez, L., Janigro, D., Ethell, D.W., 2009. Matrix metalloproteinase-7 facilitates immune access to the CNS in experimental autoimmune encephalomyelitis. BMC Neurosci. 10, 17. 
Buhmeida, A., Bendardaf, R., Hilska, M., Collan, Y., Laato, M., Syrjänen, S., Syrjänen, K., Pyrhönen, S., 2009. Prognostic significance of matrix metalloproteinase-9 (MMP-9) in stage II colorectal carcinoma. J. Gastrointest. Cancer 40, 91-97.

Bujak, M., Dobaczewski, M., Chatila, K., Mendoza, L.H., Li, N., Reddy, A., Frangogiannis, N.G., 2008. Interleukin-1 receptor type I signaling critically regulates infarct healing and cardiac remodeling. Am. J. Pathol. 173, 57-67.

Burke, B., 2004. The role of matrix metalloproteinase 7 in innate immunity 2004. Immunobiology 209, 51-56.

Burrage, P.S., Mix, K.S., Brinckerhoff, C.E., 2006. Matrix metalloproteinases: role in arthritis. Front. Biosci. 11, 529-543.

Butoi Dragomir, E., Gan, A.M., Manduteanu, I., Stan, D., Calin, M., Pirvulescu, M., Koenen, R.R., Weber, C., Simionescu, M., 2011. Cross talk between smooth muscle cells and monocytes/activated monocytes via CX3CL1/CX3CR1 axis augments expression of pro-atherogenic molecules. Biochim. Biophys. Acta. 1813, 2026-2035.

Bylander, J., Li, Q., Ramesh, G., Zhang, B., Reeves, W.B., Bond, J.S., 2008. Targeted disruption of the meprin metalloproteinase beta gene protects against renal ischemia-reperfusion injury in mice. Am. J. Physiol. Renal Physiol. 294, F480-F490.

Byun, D., Mohan, S., Yoo, M., Sexton, C., Baylink, D.J., Qin, X., 2001. Pregnancy-associated plasma protein-A accounts for the insulin-like growth factor (IGF)binding protein-4 (IGFBP-4) proteolytic activity in human pregnancy serum and enhances the mitogenic activity of IGF by degrading IGFBP-4 in vitro. J. Clin. Endocrinol. Metab. 86, 847-854.

Caceres, L.C., Bonacci, G.R., Sanchez, M.C., Chiabrando, G.A., 2010. Activated $\alpha(2)$ macroglobulin induces matrix metalloproteinase 9 expression by lowdensity lipoprotein receptor-related protein 1 through MAPK-ERK1/2 and NF- $\mathrm{KB}$ activation in macrophage-derived cell lines. J. Cell. Biochem. 111, 607617.

Cai, H., Babic, I., Wei, X., Huang, J., Witte, O.N., 2011. Invasive prostate carcinoma driven by c-Src and androgen receptor synergy. Cancer Res. 71, $862-872$. Campbell, D.J., 2003. Vasopeptidase inhibition: a double-edged sword? Hypertension 41, 383-389.

Cao, J., Rehemtulla, A., Bahou, W., Zucker, S., 1996. Membrane type matrix metalloproteinase 1 activates pro-gelatinase A without furin cleavage of the Nterminal domain. J. Biol. Chem. 271, 30174-30180.

Cao, J., Zhu, T., Lu, L., Geng, L., Wang, L., Zhang, Q., Yang, K., Wang, H., Shen, W., 2011. Estrogen induces cardioprotection in male C57BL/6J mice after acute myocardial infarction via decreased activity of matrix metalloproteinase-9 and increased Akt-Bcl-2 anti-apoptotic signaling. Int. J. Mol. Med. 28, 231237.

Capell, B.C., Collins, F.S., 2006. Human laminopathies: nuclei gone genetically awry. Nat. Rev. Genet. 7, 940-952.

Capell, B.C., Collins, F.S., Nabel, E.G., 2007. Mechanisms of cardiovascular disease in accelerated aging syndromes. Circ. Res. 101, 13-26.

Carl-McGrath, S., Lendeckel, U., Ebert, M., Roessner, A., Rocken, C., 2005. The disintegrin-metalloproteinases ADAM9, ADAM12, and ADAM15 are upregulated in gastric cancer. Int. J. Oncol. 26, 17-24.

Carrel, S., Schmidt-Kessen, A., Mach, J.P., Heumann, D., Girardet, C., 1983. Expression of common acute lymphoblastic leukemia antigen (CALLA) on human malignant melanoma cell lines. J. Immunol. 130, 2456-2460.

Carrell, T.W., Burnand, K.G., Wells, G.M., Clements, J.M., Smith, A., 2002. Stromelysin-1 (matrix metalloproteinase-3) and tissue inhibitor of metalloproteinase-3 are overexpressed in the wall of abdominal aortic aneurysms. Circulation 105, 477-482.

Carty, D.M., Delles, C., Dominiczak, A.F., 2008. Novel biomarkers for predicting preeclampsia. Trends Cardiovasc. Med. 18, 186-194.

Cataldo, D.D., Tournoy, K.G., Vermaelen, K., Munaut, C., Foidart, J.M., Louis, R., Noel, A., Pauwels, R.A., 2002. Matrix metalloproteinase-9 deficiency impairs cellular infiltration and bronchial hyperresponsiveness during allergen-induced airway inflammation. Am. J. Pathol. 161, 491-498.

Cataldo, D.D., Gueders, M.M., Rocks, N., Sounni, N.E., Evrard, B., Bartsch, P., Louis, R., Noel, A., Foidart, J.M., 2003. Pathogenic role of matrix metalloproteases and their inhibitors in asthma and chronic obstructive pulmonary disease and therapeutic relevance of matrix metalloproteases inhibitors. Cell. Mol. Biol. 49, 875-884.

Cauwe, B., Martens, E., Van den Steen, P.E., Proost, P., Van Aelst, I., Blockmans, D., Opdenakker, G., 2008. Adenylyl cyclase-associated protein-1/CAP1 as a biological target substrate of gelatinase B/MMP-9. Exp. Cell Res. 314, 2739-2749.

Cauwe, B., Martens, E., Proost, P., Opdenakker, G., 2009. Multidimensional degradomics identifies systemic autoantigens and intracellular matrix proteins as novel gelatinase B/MMP-9 substrates. Integr. Biol. (Camb.) 1, 404-426.

Cauwe, B., Martens, E., Sagaert, X., Dillen, C., Geurts, N., Li, S., Mertens, J., Thijs, G., Van den Steen, P.E., Heremans, H., De Vos, R., Blockmans, D., Arnold, B., Opdenakker, G., 2011. Deficiency of gelatinase B/MMP-9 aggravates lpr-induced lymphoproliferation and lupus-like systemic autoimmune disease. J. Autoimmun. 36, 239-252.

Cazorla, M., Hernandez, L., Nadal, A., Balbin, M., Lopez, J.M., Vizoso, F., Fernandez, P.L., Iwata, K., Cardesa, A., Lopez-Otin, C., Campo, E., 1998. Collagenase-3 expression is associated with advanced local invasion in human squamous cell carcinomas of the larynx. J. Pathol. 186, 144-150.

Cha, J., Auld, D.S., 1997. Site-directed mutagenesis of the active site glutamate in human matrilysin: investigation of its role in catalysis. Biochemistry 36 , 16019-16024.

Chabottaux, V., Sounni, N.E., Pennington, C.J., English, W.R., van den Brule, F., Blacher, S., Gilles, C., Munaut, C., Maquoi, E., Lopez-Otin, C., Murphy, G., Edwards, D.R., Foidart, J.F.M., Noel, A., 2006. Membrane-type 4 matrix metalloproteinase promotes breast cancer growth and metastases. Cancer Res. 66, 5165-5172.

Chabry, J., Checler, F., Vincent, J.P., Mazella, J., 1990. Colocalization of neurotensin receptors and of the neurotensin-degrading enzyme endopeptidase 24.16 in primary cultures of neurons. J. Neurosci. 10, 3916-3921.

Chaffer, C.L., Dopheide, B., McCulloch, D.R., Lee, A.B., Moseley, J.M., Thompson, E.W., Williams, E.D., 2005. Upregulated MT1-MMP/TIMP-2 axis in the TSUPr1-B1/B2 model of metastatic progression in transitional cell carcinoma of the bladder. Clin. Exp. Metastasis 22, 115-125.

Chambers, A.F., Matrisian, L.M., 1997. Changing views of the role of matrix metalloproteinases in metastasis. J. Natl. Cancer Inst. 89, 1260-1270.

Chan, K.C., Ko, J.M., Lung, H.L., Sedlacek, R., Zhang, Z.F., Luo, D.Z., Feng, Z.B., Chen, S., Chen, H., Chan, K.W., Tsao, S.W., Chua, D.T., Zabarovsky, E.R., Stanbridge, E.J., Lung, M.L., 2011. Catalytic activity of matrix metalloproteinase-19 is essential for tumor suppressor and anti-angiogenic activities in nasopharyngeal carcinoma. Int. J. Cancer 129, 1826-1837.

Chandler, S., Cossins, J., Lury, J., Wells, G., 1996. Macrophage metalloelastase degrades matrix and myelin proteins and processes a tumour necrosis factoralpha fusion protein. Biochem. Biophys. Res. Commun. 228, 421-429.

Chang, J.H., Javier, J.A., Chang, G.Y., Oliveira, H.B., Azar, D.T., 2005. Functional characterization of neostatins, the MMP-derived, enzymatic cleavage products of type XVIII collagen. FEBS Lett. 579, 3601-3606.

Chang, Y.H., Lin, I.L., Tsay, G.J., Yang, S.C., Yang, T.P., Ho, K.T., Hsu, T.C., Shiau, M.Y., 2008. Elevated circulatory MMP-2 and MMP-9 levels and activities in patients with rheumatoid arthritis and systemic lupus erythematosus. Clin. Biochem. 41, 955-959.

Chang, H.J., Yang, M.J., Yang, Y.H., Hou, M.F., Hsueh, E.J., Lin, S.R., 2009. MMP13 is potentially a new tumor marker for breast cancer diagnosis. Oncol. Rep. 22, 1119-1127.

Chantrain, C.F., Shimada, H., Jodele, S., Groshen, S., Ye, W., Shalinsky, D.R., Werb, Z., Coussens, L.M., DeClerck, Y.A., 2004. Stromal matrix metalloproteinase-9 regulates the vascular architecture in neuroblastoma by promoting pericyte recruitment. Cancer Res. 64, 1675-1686.

Chapman, R.E., Scott, A.A., Deschamps, A.M., Lowry, A.S., Stroud, R.E., Ikonomidis, J.S., Spinale, F.G., 2003. Matrix metalloproteinase abundance in human myocardial fibroblasts: effects of sustained pharmacologic matrix metalloproteinase inhibition. J. Mol. Cell. Cardiol. 35, 539-548.

Charbonneau, M., Harper, K., Grondin, F., Pelmus, M., McDonald, P.P., Dubois, C.M., 2007. Hypoxia-inducible factor mediates hypoxic and tumor necrosis factor alpha-induced increases in tumor necrosis factor-alpha converting enzyme/ADAM17 expression by synovial cells. J. Biol. Chem. 282, 3371433724.

Chaudhary, A.K., Singh, M., Bharti, A.C., Asotra, K., Sundaram, S., Mehrotra, R., 2010. Genetic polymorphisms of matrix metalloproteinases and their inhibitors in potentially malignant and malignant lesions of the head and neck. J. Biomed. Sci. 17, 1-13.

Checler, F., Barelli, H., Dauch, P., Dive, V., Vincent, J.P., 1995. Neurolysin: purification and assays. Methods Enzymol. 248, 593-614. 
Chen, E.I., Kridel, S.J., Howard, E.W., Li, W., Godzik, A., Smith, J.W., 2002. A unique substrate recognition profile for matrix metalloproteinase-2. J. Biol. Chem. 277, 4485-4491.

Chen, E.I., Li, W., Godzik, A., Howard, E.W., Smith, J.W., 2003. A residue in the S2 subsite controls substrate selectivity of matrix metalloproteinase-2 and matrix metalloproteinase-9. J. Biol. Chem. 278, 17158-17163.

Chen, Y.E., 2004. MMP-12, an old enzyme plays a new role in the pathogenesis of rheumatoid arthritis? Am. J. Pathol. 165, $1069-1070$.

Chen, W., Hartman, R., Ayer, R., Marcantonio, S., Kamper, J., Tang, J., Zhang, J.H., 2009. Matrix metalloproteinases inhibition provides neuroprotection against hypoxia-ischemia in the developing brain. J. Neurochem. 111, 726-736.

Chen, L., Di, D., Luo, G., Zheng, L., Tan, Y., Zhang, X., Xu, N., 2010. Immunochemical staining of MT2-MMP correlates positively to angiogenesis of human esophageal cancer. Anticancer Res. 30, 4363-4368.

Chen, Y., Nixon, N.B., Dawes, P.T., Mattey, D.L., in press. Influence of variations across the MMP-1 and -3 genes on the serum levels of MMP-1 and -3 and disease activity in rheumatoid arthritis. Genes Immun.

Chen, K.C., Wang, Y.S., Hu, C.Y., Chang, W.C., Liao, Y.C., Dai, C.Y., Juo, S.H., 2011a. OxLDL up-regulates microRNA-29b, leading to epigenetic modifications of MMP-2/MMP-9 genes: a novel mechanism for cardiovascular diseases. FASEB J. 25, 1718-1728.

Chen, K., Pociask, D.A., McAleer, J.P., Chan, Y.R., Alcorn, J.F., Kreindler, J.L., Keyser, M.R., Shapiro, S.D., Houghton, A., Kolls, J.K., Zheng, M., 2011b. IL-17RA is required for CCL2 expression, macrophage recruitment, and emphysema in response to cigarette smoke. PLoS ONE 6, e20333.

Cheng, C., Tempel, D., van Haperen, R., van Damme, L., Algür, M., Krams, R., de Crom, R., 2009. Activation of MMP8 and MMP13 by angiotensin II correlates to severe intra-plaque hemorrhages and collagen breakdown in atherosclerotic lesions with a vulnerable phenotype. Atherosclerosis $204,26-33$.

Chetty, C., Lakka, S.S., Bhoopathi, P., Kunigal, S., Geiss, R., Rao, J.S., 2008. Tissue inhibitor of metalloproteinase 3 suppresses tumor angiogenesis in matrix metalloproteinase 2-down-regulated lung cancer. Cancer Res. 68, 4736-4745.

Chetty, C., Lakka, S.S., Bhoopathi, P., Rao, J.S., 2010. MMP-2 alters VEGF expression via alphaVbeta3 integrin-mediated PI3K/AKT signaling in A549 lung cancer cells. Int. J. Cancer 127, 1081-1095.

Chiao, Y.A., Zamilpa, R., Lopez, E.F., Dai, Q., Escobar, G.P., Hakala, K., Weintraub, S.T., Lindsey, M.L., 2010. In vivo matrix metalloproteinase-7 substrates identified in the left ventricle post-myocardial infarction using proteomics. J. Proteome Res. 9, 2649-2657.

Chiba, Y., Yu, Y., Sakai, H., Misawa, M., 2007. Increase in the expression of matrix metalloproteinase-12 in the airways of rats with allergic bronchial asthma. Biol. Pharm. Bull. 30, 318-323.

Chin, J.R., Murphy, G., Werb, Z., 1985. Stromelysin, a connective tissue degrading metalloendopeptidase secreted by stimulated rabbit synovial fibroblasts in parallel with collagenase. Biosynthesis, isolation, characterization, and substrates. J. Biol. Chem. 260, 12367-12376.

Cho, C., Bunch, D.O., Faure, J.E., Goulding, E.H., Eddy, E.M., Primakoff, P., Myles, D.G., 1998. Fertilization defects in sperm from mice lacking fertilin beta. Science 281, 1857-1859.

Cho, B.S., Roelofs, K.J., Ford, J.W., Henke, P.K., Upchurch Jr., G.R., 2010. Decreased collagen and increased matrix metalloproteinase-13 in experimental abdominal aortic aneurysms in males compared with females. Surgery 147, 258-267.

Choi, D.H., Kim, Y.J., Kim, Y.G., Joh, T.H., Beal, M.F., Kim, Y.S., 2011. Role of matrix metalloproteinase 3-mediated alpha-synuclein cleavage in dopaminergic cell death. J. Biol. Chem. 286, 14168-14177.

Choi, B.D., Jeong, S.J., Wang, G., Park, J.J., Lim, D.S., Kim, B.H., Cho, Y.I., Kim, C.S., Jeong, M.J., in press. Secretory leukocyte protease inhibitor is associated with MMP-2 and MMP-9 to promote migration and invasion in SNU638 gastric cancer cells. Int. J. Mol. Med.

Choke, E., Cockerill, G., Wilson, W.R., Sayed, S., Dawson, J., Loftus, I., Thompson, M.M., 2005. A review of biological factors implicated in abdominal aortic aneurysm rupture. Eur. J. Vasc. Endovasc. Surg. 30, 227-244.

Chu, D., Zhao, Z., Zhou, Y., Li, Y., Li, J., Zheng, J., Zhao, Q., Wang, W., in press. Matrix metalloproteinase-9 is associated with relapse and prognosis of patients with colorectal cancer. Ann. Surg. Oncol.

Chung, L., Shimokawa, K., Dinakapardian, D., Grams, F., Fields, G.B., Nagase, H., 2000. Identification of the (183)RWTNNFREY(191) region as a critical segment of matrix metalloproteinase 1 for the expression of collagenolytic activity. J. Biol. Chem. 275, 29610-29617.

Chung, L., Dinakarpandian, D., Yoshida, N., Lauer-Fields, J.L., Fields, G.B., Visse, R., Nagase, H., 2004. Collagenase unwinds triple-helical collagen prior to peptide bond hydrolysis. EMBO J. 23, 3020-3030.

Churg, A., Zay, K., Shay, S., Xie, C., Shapiro, S.D., Hendricks, R., Wright, J.L., 2002. Acute cigarette smoke-induced connective tissue breakdown requires both neutrophils and macrophage metalloelastase in mice. Am. J. Respir. Cell Mol. Biol. 27, 368-374.

Churg, A., Wang, R.D., Tai, H., Wang, X., Xie, C., Dai, J., Shapiro, S.D., Wright, J.L., 2003. Macrophage metalloelastase mediates acute cigarette smoke-induced inflammation via TNF- $\alpha$ release. Am. J. Respir. Crit. Care Med. 167, 1083-1089.

Churg, A., Wang, R.D., Tai, H., Wang, X., Xie, C., Wright, J.L., 2004. Tumor necrosis factor- $\alpha$ drives $70 \%$ of cigarette smoke-induced emphysema in the mouse. Am. J. Respir. Crit. Care Med. 170, 492-498.

Churg, A., Wang, R., Wang, X., Onnervik, P., Thim, K., Wright, J.L., 2007. Effect of an MMP-9/MMP-12 inhibitor on smoke-induced emphysema and airway remodelling in guinea pigs. Thorax 62, 706-713.

Clements, K.M., Price, J.S., Chambers, M.G., Visco, D.M., Poole, A.R., Mason, R.M., 2003. Gene deletion of either interleukin-1beta, interleukin-1betaconverting enzyme, inducible nitric oxide synthase, or stromelysin 1 accelerates the development of knee osteoarthritis in mice after surgical transection of the medial collateral ligament and partial medial meniscectomy. Arthritis Rheum. 48, 3452-3463.

Clements, K.M., Flannelly, J.K., Tart, J., Brockbank, S.M., Wardale, J., Freeth, J., Parker, A.E., Newham, P., 2011. Matrix metalloproteinase 17 is necessary for cartilage aggrecan degradation in an inflammatory environment. Ann. Rheum. Dis. 70, 683-689.

Cleutjens, J.P., Creemers, E.E., 2002. Integration of concepts: cardiac extracellular matrix remodeling after myocardial infarction. J. Card. Fail. 8, S344-S348.

Colige, A., Nuytinck, L., Hausser, I., van Essen, A.J., Thiry, M., Herens, C., Ades, L.C., Malfait, F., Paepe, A.D., Franck, P., Wolff, G., Oosterwijk, J.C., Smitt, J.H., Lapière, C.M., Nusgens, B.V., 2004. Novel types of mutation responsible for the dermatosparactic type of Ehlers-Danlos syndrome (Type VIIC) and common polymorphisms in the ADAMTS2 gene. J. Invest. Dermatol. 123, 656-663.

Collier, I.E., Saffarian, S., Marmer, B.L., Elson, E.L., Goldberg, G., 2001. Substrate recognition by gelatinase A: the C-terminal domain facilitates surface diffusion. Biophys. J. 81, 2370-2377.

Colotti, C., Angeli, V., Del Ry, S., Maltinti, M., Vittorini, S., Giannessi, D., 2007. Matrix metalloprotease-2 and -9 concentration and activity in serum and culture medium samples: a methodological reappraisal. Clin. Chem. Lab. Med. 45, 1292-1298.

Comabella, M., Rio, J., Espejo, C., Ruiz de Villa, M., Al-Zayat, H., Nos, C., Deisenhammer, F., Baranzini, S.E., Nonell, L., Lopez, C., Julia, E., Oksenberg, J.R., Montalban, X., 2009. Changes in matrix metalloproteinases and their inhibitors during interferon-beta treatment in multiple sclerosis. Clin. Immunol. $130,145-150$.

Conant, K., McArthur, J.C., Griffin, D.E., Sjulson, L., Wahl, L.M., Irani, D.N., 1999. Cerebrospinal fluid levels of MMP-2, 7, and 9 are elevated in association with human immunodeficiency virus dementia. Ann. Neurol. 46, 391-398.

Conover, C.A., 2010. PAPP-A: a new anti-aging target? Aging Cell 9, 942-946.

Conover, C.A., Boldt, H.B., Bale, L.K., Clifton, K.B., Grell, J.A., Mader, J.R., Mason, E.J., Powell, D.R., 2011. Pregnancy-associated plasma protein-A2 (PAPP-A2): tissue expression and biological consequences of gene knockout in mice. Endocrinology 152, 2837-2844.

Consuegra-Sanchez, L., Fredericks, S., Kaski, J.C., 2009a. Pregnancy-associated plasma protein-A (PAPP-A) and cardiovascular risk. Atherosclerosis 203, 346352.

Consuegra-Sanchez, L., Fredericks, S., Kaski, J.C., 2009b. Pregnancy-associated plasma protein A: has this biomarker crossed the boundary from research to clinical practice? Drug News Perspect. 22, 341-348.

Copin, J.C., Bengualid, D.J., Da Silva, R.F., Kargiotis, O., Schaller, K., Gasche, Y., 2011. Recombinant tissue plasminogen activator induces blood-brain barrier breakdown by a matrix metalloproteinase-9-independent pathway after transient focal cerebral ischemia in mouse. Eur. J. Neurosci. 34, 1085-1092. 
Cosgrove, G.P., Schwarz, M.I., Geraci, M.W., Brown, K.K., Worthen, G.S., 2002. Overexpression of matrix metalloproteinase-7 in pulmonary fibrosis. Chest $121,25 S-26 S$.

Coulson-Thomas, V.J., Gesteira, T.F., Coulson-Thomas, Y.M., Vicente, C.M., Tersariol, I.L., Nader, H.B., Toma, L., 2010. Fibroblast and prostate tumor cell crosstalk: fibroblast differentiation, TGF- $\beta$, and extracellular matrix down-regulation. Exp. Cell Res. 316, 3207-3226.

Coussens, L.M., Werb, Z., 1996. Matrix metalloproteinases and the development of cancer. Chem. Biol. 3, 895-904.

Coussens, L.M., Tinkle, C.L., Hanahan, D., Werb, Z., 2000. MMP-9 supplied by bone marrow-derived cells contributes to skin carcinogenesis. Cell 103, 481490.

Coussens, L., Fingleton, B., Matrisian, L.N., 2002. Matrix metalloproteinase inhibitors and cancer: trials and tribulations. Science 295, $2387-2392$.

Culpitt, S.V., Rogers, D.F., Traves, S.L., Barnes, P.J., Donnelly, L.E., 2005. Sputum matrix metalloproteases: comparison between chronic obstructive pulmonary disease and asthma. Respir. Med. 99, 703-710.

Curci, J.A., Liao, S., Huffman, M.D., Shapiro, S.D., Thompson, R.W., 1998. Expression and localization of macrophage elastase (matrix metalloproteinase-12) in abdominal aortic aneurysms. J. Clin. Invest. 102, 1900-1910.

Cyr, N.E., Kua, L.H., Bruce, L.A., Chadwick, J.G., Tetel, M.J., Wolfson, A.J., 2010. Nuclear thimet oligopeptidase is coexpressed with oestrogen receptor alpha in hypothalamic cells and regulated by oestradiol in female mice. J. Neuroendocrinol. 22, 936-943.

Daniele, A., Zito, A.F., Giannelli, G., Divella, R., Asselti, M., Mazzocca, A., Paradiso, A., Quaranta, M., 2010. Expression of metalloproteinases MMP-2 and MMP9 in sentinel lymph node and serum of patients with metastatic and non-metastatic breast cancer. Anticancer Res. 30, 3521-3527.

Danielsen, P.L., Holst, A.V., Maltesen, H.R., Bassi, M.R., Holst, P.J., Heinemeier, K.M., Olsen, J., Danielsen, C.C., Poulsen, S.S., Jorgensen, L.N., Agren, M.S., 2011. Matrix metalloproteinase-8 overexpression prevents proper tissue repair. Surgery 150 (5), 897-906.

D’Armiento, J., Dalal, S.S., Okada, Y., Berg, R.A., Chada, K., 1992. Collagenase expression in the lungs of transgenic mice causes pulmonary emphysema. Cell 71, 955-961.

Daull, P., Jeng, A.Y., Battistini, B., 2007. Towards triple vasopeptidase inhibitors for the treatment of cardiovascular diseases. J. Cardiovasc. Pharmacol. 50, 247-256.

Davidson, B., Goldberg, I., Gotlieb, W.H., Kopolovic, J., Ben Baruch, G., Nesland, J.M., Reich, R., 2002. The prognostic value of metalloproteinases and angiogenic factors in ovarian carcinoma. Mol. Cell. Endocrinol. 187, 39-45.

Davidson, R.K., Waters, J.G., Kevorkian, L., Darrah, C., Cooper, A., Donell, S.T., Clark, I.M., 2006. Expression profiling of metalloproteinases and their inhibitors in synovium and cartilage. Arthritis Res. Ther. 8, R124.

Davies, S., Dai, D., Pickett, G., Thiel, K.W., Korovkina, V.P., Leslie, K.K., 2011. Effects of bevacizumab in mouse model of endometrial cancer: defining the molecular basis for resistance. Oncol. Rep. 25, 855-862.

Davis, T.P., Gillespie, T.J., Konings, P.N.M., 1992. Specificity of neurotensin metabolism by regional rat brain slices. J. Neurochem. 58, 608-617.

Deb, S., Wenjun Zhang, J., Gottschall, P.E., 2003. Beta-amyloid induces the production of active, matrix-degrading proteases in cultured rat astrocytes. Brain Res. 970, 205-213.

Decock, J., Hendrickx, W., Vanleeuw, U., Van Belle, V., Van Huffel, S., Christiaens, M.R., Ye, S., Paridaens, R., 2008. Plasma MMP1 and MMP8 expression in breast cancer: protective role of MMP8 against lymph node metastasis. BMC Cancer 8, 77.

De Coignac, A.B., Elson, G., Delneste, Y., Magistrelli, G., Jeannin, P., Aubry, J.P., Berthier, O., Schmitt, D., Bonnefoy, J.Y., Gauchat, J.F., 2000. A novel matrilysin-like proteinase. Eur. J. Biochem. 267, 3323-3329.

Deguchi, J.O., Aikawa, E., Libby, P., Vachon, J.R., Inada, M., Krane, S.M., Whittaker, P., Aikawa, M., 2005. Matrix metalloproteinase-13/collagenase-3 deletion promotes collagen accumulation and organization in mouse atherosclerotic plaques. Circulation 112, 2708-2715.

Dehan, E., Ben-Dor, A., Liao, W., Lipson, D., Frimer, H., Rienstein, S., Simansky, D., Krupsky, M., Yaton, P., Friedman, E., Rechavi, G., Perlman, M., AviramGoldring, A., Izraeli, S., Bittner, M., Yakhini, Z., Kaminski, N., 2007. Chromosomal aberrations and gene expression profiles in non-small cell lung cancer. Lung Cancer 56, 175-184.

Delassus, G.S., Cho, H., Eliceiri, G.L., 2011. New signaling pathways from cancer progression modulators to mRNA expression of matrix metalloproteinases in breast cancer cells. Cell Physiol. 226 (12), 3378-3384.

Del Zoppo, G.J., Milner, R., Mabuchi, T., Hung, S., Wang, X., Berg, G.I., Koziol, J.A., 2007. Microglial activation and matrix protease generation during focal cerebral ischemia. Stroke 38, 646-651.

Demedts, I.K., Morel-Montero, A., Lebecque, S., Pacheco, Y., Cataldo, D., Joos, G.F., Pauwels, R.A., Brusselle, G.G., 2006. Elevated MMP-12 protein levels in induced sputum from patients with COPD. Thorax 61, 196-201.

Denecke, J., Brune, T., Feldhaus, T., Robenek, H., Kranz, C., Auchus, R.J., Agarwal, A.K., Marquardt, T., 2006. A homozygous ZMPSTE24 null mutation in combination with a heterozygous mutation in the LMNA gene causes Hutchinson-Gilford progeria syndrome (HGPS): insights into the pathophysiology of HGPS. Hum. Mutat. 27, 524-531.

Deng, Y.P., Li, W., Li, Y.L., Xu, H., Liang, S.S., Zhang, L.H., Li, Y.L., 2009. MT1-MMP up-regulates VEGF expression in human breast carcinoma MCF-7 cells and induces tumor angiogenesis. Zhonghua Zhong Liu Za Zhi 31, 727-731.

Denny, M.F., Chandaroy, P., Killen, P.D., Caricchio, R., Lewis, E.E., Richardson, B.C., Lee, K.D., Gavalchin, J., Kaplan, M.J., 2006. Accelerated macrophage apoptosis induces autoantibody formation and organ damage in systemic lupus erythematosus. J. Immunol. 176, $2095-2104$.

D’Ercole, A.J., Ye, P., O’Kusky, J.R., 2002. Mutant mouse models of insulin-like growth factor actions in the central nervous system. Neuropeptides 36, 209220.

Deryugina, E.I., Soroceanu, L., Strongin, A.Y., 2002a. Up-regulation of vascular endothelial growth factor by membrane-type 1 matrix metalloproteinase stimulates human glioma xenograft growth and angiogenesis. Cancer Res. 62, 580-588.

Deryugina, E., Ratnikov, B.I., Postnova, T.I., Rozanov, D.V., Strongin, A.Y., 2002b. Processing of integrin alpha(v) subunit by membranetype 1 matrix metalloproteinase stimulates migration of breast carcinoma cells on vitronectin and enhances tyrosine phosphorylation of focal adhesion kinase. J. Biol. Chem. 277, 9749-9756.

Deryugina, E.I., Quigley, J.P., 2006. Matrix metalloproteinases and tumor metastasis. Cancer Metastasis Rev. 25, 9-34.

Deshmukh, H.S., McLachlan, A., Atkinson, J.J., Hardie, W.D., Korfhagen, T.R., Dietsch, M., Liu, Y., Di, P.Y., Wesselkamper, S.C., Borchers, M.T., Leikauf, G.D., 2009. Matrix metalloproteinase-14 mediates a phenotypic shift in the airways to increase mucin production. Am. J. Respir. Crit. Care Med. 180, 834-845.

Devarajan, P., Mookhtiar, K., van Wart, H., Berliner, N., 1991. Structure and expression of the cDNA encoding human neutrophil collagenase. Blood 77, 27312738.

Diaz-Sanchez, M., Williams, K., DeLuca, G.C., Esiri, M.M., 2006. Protein co-expression with axonal injury in multiple sclerosis plaques. Acta Neuropathol. 111, 289-299.

Di Bella, D., Lazzaro, F., Brusco, A., Plumari, M., Battaglia, G., Pastore, A., Finardi, A., Cagnoli, C., Tempia, F., Frontali, M., Veneziano, L., Sacco, T., Boda, E., Brussino, A., Bonn, F., Castellotti, B., Baratta, S., Mariotti, C., Gellera, C., Fracasso, V., Magri, S., Langer, T., Plevani, P., Di Donato, S., Muzi-Falconi, M., Taroni, F., 2010. Mutations in the mitochondrial protease gene AFG3L2 cause dominant hereditary ataxia SCA28. Nat. Genet. 42, 313-321.

Doerner, A.M., Zuraw, B.L., 2009. TGF-beta1 induced epithelial to mesenchymal transition (EMT) in human bronchial epithelial cells is enhanced by IL-1beta but not abrogated by corticosteroids. Respir. Res. 10, 100.

Doi, Y., Mizuno, T., Maki, Y., Jin, S., Mizoguchi, H., Ikeyama, M., Doi, M., Michikawa, M., Takeuchi, H., Suzumura, A., 2009. Microglia activated with the toll-like receptor 9 ligand CpG attenuate oligomeric amyloid \{beta neurotoxicity in in vitro and in vivo models of Alzheimer's disease. Am. J. Pathol. 175, 21212132.

Domagala-Kulawik, J., Maskey-Warzechowska, M., Kraszewska, I., Chazan, R., 2003. The cellular composition and macrophage phenotype in induced sputum in smokers and ex-smokers with COPD. Chest 123, 1054-1059.

Dong, Z., Kumar, R., Yang, X., Fidler, I.J., 1997. Macrophage-derived metalloelastase is responsible for the generation of angiostatin in Lewis lung carcinoma. Cell 88, 801-810. 
Dong, Z., Katar, M., Alousi, S., Berk, R.S., 2001. Expression of membrane-type matrix metalloproteinases 4, 5, and 6 in mouse corneas infected with P. aeruginosa. Invest. Ophthalmol. Vis. Sci. 42, 3223-3227.

Du, R., Petritsch, C., Lu, K., Liu, P., Haller, A., Ganss, R., Song, H., Vandenberg, S., Bergers, G., 2008. Matrix metalloproteinase-2 regulates vascular patterning and growth affecting tumor cell survival and invasion in GBM. Neuro Oncol. 10, 254-264.

Du, S., Sandoval, F., Trinh, P., Voskuhl, R.R., 2010. Additive effects of combination treatment with anti-inflammatory and neuroprotective agents in experimental autoimmune encephalomyelitis. J. Neuroimmunol. 219, 64-74.

Dubois, B., Masure, S., Hurtenbach, U., Paemen, L., Heremans, H., van den Oord, J., Sciot, R., Meinhardt, T., Hämmerling, G., Opdenakker, G., Arnold, B., 1999. Resistance of young gelatinase B-deficient mice to experimental autoimmune encephalomyelitis and necrotizing tail lesions. J. Clin. Invest. 104, 15071515.

Duffy, M.J., Maguire, T.M., Hill, A., McDermott, E., O’Higgins, N., 2000. Breast Cancer Res. 2, 252-257.

Dufour, A., Zucker, S., Sampson, N.S., Kuscu, C., Cao, J., 2010. Role of matrix metalloproteinase-9 dimers in cell migration: design of inhibitory peptides. J. Biol. Chem. 285, 35944-35956.

Dumin, J.A., Dickeson, S.K., Stricker, T.P., Bhattacharyya-Pakrasi, M., Roby, J.D., Santoro, S.A., Parks, W.C., 2001. Pro-collagenase-1 (matrix metalloproteinase1) binds the alpha(2)beta(1) integrin upon release from keratinocytes migrating on type I collagen. J. Biol. Chem. 276, $29368-29374$.

Dunn, J.R., Reed, J.E., du Plessis, D.G., Shaw, E.J., Reeves, P., Gee, A.L., Warnke, P., Walker, C., 2006. Expression of ADAMTS-8, a secreted protease with antiangiogenic properties, is downregulated in brain tumours. Br. J. Cancer 94, 1186-1193.

Durham, S.K., Kiefer, M.C., Riggs, B.L., Conover, C.A., 1994. Regulation of insulin-like growth factor binding protein 4 by a specific insulin-like growth factor binding protein 4 proteinase in normal human osteoblast-like cells: implications in bone cell physiology. J. Bone Miner. Res. 9 , $111-117$.

Eckman, E.A., Reed, D.K., Eckman, C.B., 2001. Degradation of the Alzheimer's amyloid peptide by endothelin-converting enzyme. J. Biol. Chem. 276, 2454024548.

Eckman, E.A., Watson, M., Marlow, L., Sambamurti, K., Eckman, C.B., 2003. Alzheimer's disease $\beta$-amyloid peptide is increased in mice deficient in endothelin-converting enzyme. J. Biol. Chem. 278, 2081-2084

Edwards, D.R., Handsley, M.M., Pennington, C.J., 2008. The ADAM metalloproteinases. Mol. Aspects Med. 29, 258-289.

Egeblad, M., Werb, Z., 2002. New functions for the matrix metalloproteinases in cancer progression. Nat. Rev. Cancer 2, 161-174.

Ehrenfeld, P., Conejeros, I., Pavicic, M.F., Matus, C.E., Gonzalez, C.B., Quest, A.F., Bhoola, K.D., Poblete, M.T., Burgos, R.A., Figueroa, C.D., 2011. Activation of kinin B1 receptor increases the release of metalloproteases-2 and -9 from both estrogen-sensitive and -insensitive breast cancer cells. Cancer Lett. 301 , 106-118.

Eliason, J.L., Hannawa, K.K., Ailawadi, G., Sinha, I., Ford, J.W., Deogracias, M.P., Roelofs, K.J., Woodrum, D.T., Ennis, T.L., Henke, P.K., Stanley, J.C., Thompson, R.W., Upchurch Jr., G.R., 2005. Neutrophil depletion inhibits experimental abdominal aortic aneurysm formation. Circulation 112, 232-240.

Elkington, P.T., Nuttall, R.K., Boyle, J.J., O'Kane, C.M., Horncastle, D.E., Edwards, D.R., Friedland, J.S., 2005. Mycobacterium tuberculosis, but not vaccine BCG, specifically upregulates matrix metalloproteinase-1. Am. J. Respir. Crit. Care Med. 172, 1596-1604.

Elkington, P., Shiomi, T., Breen, R., Nuttall, R.K., Ugarte-Gil, C.A., Walker, N.F., Saraiva, L., Pedersen, B., Mauri, F., Lipman, M., Edwards, D.R., Robertson, B.D., D’Armiento, J., Friedland, J.S., 2011. MMP-1 drives immunopathology in human tuberculosis and transgenic mice. J. Clin. Invest. 121, 1827-1833.

Elkins, P.A., Ho, Y.S., Smith, W.W., Janson, C.A., D’Alessio, K.J., McQueney, M.S., Cummings, M.D., Romanic, A.M., 2002. Structure of the C-terminally truncated human ProMMP9, a gelatin-binding matrix metalloproteinase. Acta Crystallogr. D Biol. Crystallogr. 58, 1182-1192.

Ellenrieder, V., Alber, B., Lacher, U., Hendler, S.F., Menke, A., Boeck, W., Wagner, M., Wilda, M., Friess, H., Büchler, M., Adler, G., Gress, T.M., 2000. Role of MTMMPs and MMP-2 in pancreatic cancer progression. Int. J. Cancer 85, 14-20.

Elnemr, A., Yonemura, Y., Bandou, E., Kinoshita, K., Kawamura, T., Takahashi, S., Tochiori, S., Endou, Y., Sasaki, T., 2003. Expression of collagenase-3 (matrix metalloproteinase-13) in human gastric cancer. Gastric Cancer 6, 30-38.

Emlen, W., Niebur, J., Kadera, R., 1994. Accelerated in vitro apoptosis of lymphocytes from patients with systemic lupus erythematosus. J. Immunol. 152, 3685-3692.

Emoto, N., Yanagisawa, M., 1995. Endothelin-converting enzyme-2 is a membrane-bound, phosphoramidon-sensitive metalloprotease with acidic pH optimum. J. Biol. Chem. 270, 15262-15268.

Endo, K., Takino, T., Miyamori, H., Kinsen, H., Yoshizaki, T., Furukawa, M., Sato, H., 2003. Cleavage of syndecan-1 by membrane type matrix metalloproteinase-1 stimulates cell migration. J. Biol. Chem. 278, 40764-40770.

England, K.A., Price, A.P., Tram, K.V., Shapiro, S.D., Blazar, B.R., Panoskaltsis-Mortari, A., 2011. Evidence for early fibrosis and increased airway resistance in bone marrow transplant recipient mice deficient in MMP12. Am. J. Physiol. Lung Cell. Mol. Physiol. 301, L519-L526.

English, W.R., Puente, X.S., Freije, J.M., Knäuper, V., Amour, A., Merryweather, A., Lopez-Otin, C., Murphy, G., 2000. metalloproteinase (MMP-17) has tumor necrosis factor-alpha convertase activity but does not activate pro-MMP2. J. Biol. Chem. 275, 14046-14055.

English, W.R., Velasco, G., Stracke, J.O., Knäuper, V., Murphy, G., 2001. Catalytic activities of membrane-type 6 matrix metalloproteinase (MMP-25). FEBS Lett. 491, 137-142.

Erler, J.T., Bennewith, K.L., Cox, T.R., Lang, G., Bird, D., Koong, A., Le, Q.T., Giaccia, A.J., 2009. Hypoxia-induced lysyl oxidase is a critical mediator of bone marrow cell recruitment to form the premetastatic niche. Cancer Cell 15, 35-44.

Esser, K., Tursun, B., Ingenhoven, M., Michaelis, G., Pratje, E., 2002. A novel two-step mechanism for removal of a mitochondrial signal sequence involves the mAAA complex and the putative rhomboid protease Pcp1. J. Mol. Biol. 323, 835-843.

Ezhilarasan, R., Jadhav, U., Mohanam, I., Rao, J.S., Gujrati, M., Mohanam, S., 2009. The hemopexin domain of MMP-9 inhibits angiogenesis and retards the growth of intracranial glioblastoma xenograft in nude mice. Int. J. Cancer 124, 306-315.

Fabriek, B.O., Møller, H.J., Vloet, R.P., van Winsen, L.M., Hanemaaijer, R., Teunissen, C.E., Uitdehaag, B.M., van den Berg, T.K., Dijkstra, C.D., 2007. Proteolytic shedding of the macrophage scavenger receptor CD163 in multiple sclerosis. J. Neuroimmunol. 187, 179-186.

Fager, N., Jaworski, D.M., 2000. Differential spatial distribution and temporal regulation of tissue inhibitor of metalloproteinase mRNA expression during rat central nervous system development. Mech. Dev. 98, 105-109.

Fahrenholz, F., Postina, R., 2006. $\alpha$-Secretase activation - an approach to Alzheimer's disease therapy. Neurodegener. Dis. 3, 255-261.

Fainardi, E., Castellazzi, M., Tamborino, C., Trentini, A., Manfrinato, M.C., Baldi, E., Tola, M.R., Dallocchio, F., Granieri, E., Bellini, T., 2009. Potential relevance of cerebrospinal fluid and serum levels and intrathecal synthesis of active matrix metalloproteinase-2 (MMP-2) as markers of disease remission in patients with multiple sclerosis. Mult. Scler. 15, 547-554.

Fariñas, I., Lopez-Otin, C., 2009. Metalloproteinase MT5-MMP is an essential modulator of neuro-immune interactions in thermal pain stimulation. Proc. Natl. Acad. Sci. USA 106, 16451-16456.

Fasciglione, G.F., Marini, S., D’Alessio, S., Politi, V., Coletta, M., 2000. pH- and temperature-dependence of functional modulation in metalloproteinases. A comparison between neutrophil collagenase and gelatinases A and B. Biophys. J. 79, 2138-2149.

Fenton, K., Fismen, S., Hedberg, A., Seredkina, N., Fenton, C., Mortensen, E.S., Rekvig, O.P., 2009. Anti-dsDNA antibodies promote initiation, and acquired loss of renal Dnase1 promotes progression of lupus nephritis in autoimmune (NZBxNZW)F1 mice. PLoS One 4 (12), e8474.

Fernandes, K.S., Brum, D.G., Sandrim, V.C., Guerreiro, C.T., Barreir, A.A., Tanus-Santos, J.E., 2009. Matrix metalloproteinase-9 genotypes and haplotypes are associated with multiple sclerosis and with the degree of disability of the disease. J. Neuroimmunol. 214, $128-131$.

Fernandes, J.C., Martel-Pelletier, J., Lascau-Coman, V., Moldovan, F., Jovanovic, D., Raynauld, J.P., Pelletier, J.P., 1998. Collagenase-1 and collagenase-3 synthesis in early experimental osteoarthritic canine cartilage. An immunohistochemical study. J. Rheumatol. 25, 1585-1594.

Fernandez-Catalan, C., Bode, W., Huber, R., Turk, D., Calvete, J.J., Lichte, A., Tschesche, H., Maskos, K., 1998. Crystal structure of the complex formed by the membrane type 1-matrix metalloproteinase with the tissue inhibitor of metalloproteinases-2, the soluble progelatinase A receptor. EMBO J. 17, 52385248.

Ferrara, N., Gerber, H.P., LeCouter, J., 2003. The biology of VEGF and its receptors. Nat. Med. 9, 669-676. 
Ferreirinha, F., Quattrini, A., Pirrozi, M., Valsecchi, V., Dina, G., Broccoli, V., Auricchio, A., Piemonte, F., Tozzi, G., Gaeta, L., Casari, G., Ballabio, A., Rugarli, E.I., 2004. Axonal degeneration in paraplegin-deficient mice is associated with abnormal mitochondria and impairment of axonal transport. J. Clin. Invest. $113,231-242$.

Ferro, E.S., Carreno, F.R., Goni, C., Garrido, P.A., Guimaraes, A.O., Castro, L.M., Oliveira, V., Araujo, M.C., Rioli, V., Gomes, M.D., Fontenele-Neto, J.D., Hyslop, S., 2004. The intracellular distribution and secretion of endopeptidases 24.15 (EC 3.4.24.15) and 24.16 (EC 3.4.24.16). Protein Pept. Lett. 11, 415-421.

Fields, G.B., van Wart, H.E., Birkedal-Hansen, H., 1987. Sequence specificity of human skin fibroblast collagenase. Evidence for the role of collagen structure in determining the collagenase cleavage site. J. Biol. Chem. 262, 6221-6226.

Finder, V.H., 2010. disease: a general introduction and pathomechanism. J. Alzheimers Dis. 22, 5-19.

Fingleton, B., 2006. Matrix metalloproteinases: roles in cancer and metastasis. Front. Biosci. 11, 479-491.

Fingleton, B., 2007. Matrix metalloproteinases as valid clinical targets. Curr. Pharm. Des. 13, 333-346.

Finlay, G.A., O’Driscoll, L.R., Russell, K.J., D’Arcy, E.M., Masterson, J.B., Fitzgerald, M.X., O’Connor, C.M., 1997. Matrix metalloproteinase expression and production by alveolar macrophages in emphysema. Am. J. Respir. Crit. Care Med. 156, 240-247.

Fiore, E., Fusco, C., Romero, P., Stamenkovic, I., 2002. Matrix metalloproteinase 9 (MMP-9/gelatinase B) proteolytically cleaves ICAM-1 and participates in tumor cell resistance to natural killer cell-mediated cytotoxicity. Oncogene 21, 5213-5223.

Fiotti, N., Xiong, W., Giansante, C., 2010. MMP-2 genetic variant and plaque features of instability. Atherosclerosis 210, 43-44.

Firestein, G.S., 2003. Evolving concepts of rheumatoid arthritis. Nature 423, 356-361.

Flex, A., Gaetani, E., Proia, A.S., Pecorini, G., Straface, G., Biscetti, F., Fioroni, G., Sabusco, A., Flore, R., Tondi, P., Pola, P., Pola, R., 2006. Analysis of functional polymorphisms of metalloproteinase genes in persons with vascular dementia and Alzheimer's disease. J. Gerontol. 61, 1065-1069.

Folgueras, A.R., Fueyo, A., Garcia-Suarez, O., Cox, J., Astudillo, A., Tortorella, P., Campestre, C., Gutierrez-Fernandez, A., Fanjul-Fernandez, M., Pennington, C.J., Edwards, D.R., Overall, C.M., Lopez-Otin, C., 2008. Collagenase-2 deficiency or inhibition impairs experimental autoimmune encephalomyelitis in mice. J. Biol. Chem. 283, 9465-9474.

Folgueras, A.R., Valdes-Sanchez, T., Llano, E., Menendez, L., Baamonde, A., Denlinger, B.L., Belmonte, C., Juarez, L., Lastra, A., Garcia-Suarez, O., Astudillo, A., Kirstein, M., Pendas, A.M., Fortin, C.F., Sohail, A., Sun, Q., McDonald, P.P., Fridman, R., Fülöp, T., 2010. MT6-MMP is present in lipid rafts and faces inward in living human PMNs but translocates to the cell surface during neutrophil apoptosis. Int. Immunol. 22, 637-649.

Fortin, J.P., Chinnapen, D., Beinborn, M., Lencer, W., Kopin, A.S., 2011. Discovery of sual-action membrane-anchored modulators of incretin receptors. PLoS One 6 (9), e24693.

Fosang, A.J., Last, K., Knäuper, V., Murphy, G., Neame, P.J., 1996. Degradation of cartilage aggrecan by collagenase-3 (MMP-13). FEBS Lett. 380, 17-20.

Fowlkes, J.L., Enghild, J.J., Suzuki, K., Nagase, H., 1994. Matrix metalloproteinases degrade insulin-like growth factor-binding protein-3 in dermal fibroblast cultures. J. Biol. Chem. 269, 25742-25746.

Frederick, L.A., Matthews, J.A., Jamieson, L., Justilien, V., Thompson, E.A., Radisky, D.C., Fields, A.P., 2008. Matrix metalloproteinase-10 is a critical effector of protein kinase Ciota-Par6alpha-mediated lung cancer. Oncogene 27, 4841-4853.

Freedland, S.J., Seligson, D.B., Liu, A.Y., Pantuck, A.J., Paik, S.H., Horvath, S., Wieder, J.A., Zisman, A., Nguyen, D., Tso, C.L., Palotie, A.V., Belldegrun, A.S., 2003. Loss of CD10 (neutral endopeptidase) is a frequent and early event in human prostate cancer. Prostate 55, 71-80.

Freemont, A.J., Byers, R.J., Taiwo, Y.O., Hoyland, J.A., 1999. In situ zymographic localisation of type II collagen degrading activity in osteoarthritic human articular cartilage. Ann. Rheum. Dis. 58, 357-365.

Freije, J.M., Diez-Itza, I., Balbin, M., Sanchez, L.M., Blasco, R., Tolivia, J., Lopez-Otin, C., 1994. Molecular cloning and expression of collagenase-3, a novel human matrix metalloproteinase produced by breast carcinomas. J. Biol. Chem. 269, 16766-16773.

Fujikawa, K., Suzuki, H., McMullen, B., Chung, D., 2001. Purification of human von Willebrand factor-cleaving protease and its identification as a new member of the metalloproteinase family. Blood 98, 1662-1666.

Fujita, Y., Shiomi, T., Yanagimoto, S., Matsumoto, H., Toyama, Y., Okada, Y., 2006. Tetraspanin CD151 is expressed in osteoarthritic cartilage and is involved in pericellular activation of pro-matrix metalloproteinase 7 in osteoarthritic chondrocytes. Arthritis Rheum. 54, 3233-3243.

Furlan, M., 1996. Von Willebrand factor: molecular size and functional activity. Ann. Hematol. 72, 341-348.

Furuse, M., Fujita, K., Hiiragi, T., Fujimoto, K., Tsukita, S., 1998. Claudin-1 and -2: novel integral membrane proteins localizing at tight junctions with no sequence similarity to occludin. J. Cell Biol. 141, 1539-1550.

Gaetje, R., Holtrich, U., Engels, K., Kourtis, K., Cikrit, E., Kissler, S., Rody, A., Karn, T., Kaufmann, M., 2007. Expression of membrane-type 5 matrix metalloproteinase in human endometrium and endometriosis. Gynecol. Endocrinol. 23, 567-573.

Gakh, O., Cavadini, P., Isaya, G., 2002. Mitochondrial processing peptidases. Biochim. Biophys. Acta 1592, 63-77.

Galis, Z.S., Sukhova, G.K., Kranzhofer, R., Clark, S., Libby, P., 1995. Macrophage foam cells from experimental atheroma constitutively produce matrixdegrading proteinases. Proc. Natl. Acad. Sci. USA 92, 402-406.

Galis, Z.S., Khatri, J.J., 2002. Matrix metalloproteinases in vascular remodeling and atherogenesis: the good, the bad, and the ugly. Circ. Res. 90, $251-262$.

Galis, Z.S., Johnson, C., Godin, D., Magid, R., Shipley, J.M., Senior, R.M., Ivan, E., 2002. Targeted disruption of the matrix metalloproteinase-9 gene impairs smooth muscle cell migration and geometrica arterial remodeling. Circ. Res. 91, 852-859.

Gall, A.L., Ruff, M., Kannan, R., Cuniasse, P., Yiotakis, A., Dive, V., Rio, M.C., Basset, P., Moras, D., 2001. Crystal structure of the stromelysin-3 (MMP-11) catalytic domain complexed with a phosphinic inhibitor mimicking the transition-state. J. Mol. Biol. 307, 577-586.

Galvez-Gastelum, F.J., Garcia-Bañuelos, J.J., Beas-Zarate, C., Segura-Flores, A., Gonzalez, H., Chaparro-Huerta, V., Salazar-Montes, A., Sandoval-Rodriguez, A.S., Bueno-Topete, M., Lucano-Landeros, S., Medina-Preciado, D., Gonzalez-Garcia, I., Armendariz-Borunda, J., 2011. Combinatorial gene therapy induces regression of hepatic encephalopathy. Gene Ther. 18, 88-94.

Ganji, P.C., Nalla, A.K., Gupta, R., Mohanam, S., Gujrati, M., Dinh, D.H., Raom, J.S., 2011. siRNA-mediated downregulation of MMP-9 and uPAR in combination with radiation induces G2/M cell-cycle arrest in Medulloblastoma. Mol. Cancer Res. 9, 51-66.

Garcia, S., Forteza, J., Lopez-Otin, C., Gomez-Reino, J.J., Gonzalez, A., Conde, C., 2010. Matrix metalloproteinase-8 deficiency increases joint inflammation and bone erosion in the K/BxN serum-transfer arthritis model. Arthritis Res. Ther. 12, R224.

Garcia-Montojo, M., Dominguez-Mozo, M.I., de las Heras, V., Bartolome, M., Garcia-Martinez, A., Arroyo, R., Alvarez-Lafuente, R., 2010. Neutralizing antibodies, MxA expression and MMP-9/TIMP-1 ratio as markers of bioavailability of interferon-beta treatment in multiple sclerosis patients: a twoyear follow-up study. Eur. J. Neurol. 17, 470-478.

Garcia-Prieto, E., Gonzalez-Lopez, A., Cabrera, S., Astudillo, A., Gutierrez-Fernandez, A., Fanjul-Fernandez, M., Batalla-Solis, E., Puente, X.S., Fueyo, A., LopezOtin, C., Albaiceta, G.M., 2010. Resistance to bleomycin-induced lung fibrosis in MMP-8 deficient mice is mediated by interleukin-10. PLoS One 5, e13242.

Garg, P., Sarma, D., Jeppsson, S., Patel, N.R., Gewirtz, A.T., Merlin, D., Sitaraman, S.V., 2010. Matrix metalloproteinase-9 functions as a tumor suppressor in colitis-associated cancer. Cancer Res. 70, 792-801.

Garton, K.J., Gough, P.J., Raines, E.W., 2006. Emerging roles for ectodomain shedding in the regulation of inflammatory responses. J. Leukoc. Biol. 79, 11051106.

Garvican, E.R., Vaughan-Thomas, A., Redmond, C., Clegg, P.D., 2008. MT3-MMP (MMP-16) is downregulated by in vitro cytokine stimulation of cartilage, but unaltered in naturally occurring equine osteoarthritis and osteochondrosis. Connect. Tissue Res. 49, 62-67.

Gaultier, A., Cousin, H., Darribere, T., Alfandari, D., 2002. ADAM13 disintegrin and cysteine-rich domains bind to the second heparin-binding domain of fibronectin. J. Biol. Chem. 277, 23336-23344

Gauthier, M.C., Racine, C., Ferland, C., Flamand, N., Chakir, J., Tremblay, G.M., 2003. Expression of membrane type-4 matrix metalloproteinase (metalloproteinase-17) by human eosinophils. Int. J. Biochem. Cell Biol. 35, 1667-1673.

Ge, G., Greenspan, D.S., 2006. Developmental roles of the BMP1/TLD metalloproteinases. Birth Defects Res. C Embryo Today 78, 47-68.

Genden, E.M., Molineaux, C.J., 1991. Inhibition of endopeptidase-24.15 decreases blood pressure in normotensive rats. Hypertension 18, 360-365. 
Gerstein, E.S., Sini, L., Ryabov, A.B., Dvorova, E.K., Yurchenko, A.A., Stilidi, I.S., Kushlinskii, N.E., Davydov, M.I., 2009. Comparative enzyme immunoassay of matrix metalloproteinases-2, -7, -9 and their tissue inhibitor-2 in tumors and plasma of patients with gastric cancer. Bull. Exp. Biol. Med. 148, 899-902.

Ghaderian, S.M., Akbarzadeh Najar, R., Tabatabaei Panah, A.S., 2010. Genetic polymorphisms and plasma levels of matrix metalloproteinases and their relationships with developing acute myocardial infarction. Coron. Artery Dis. 21, 330-335.

Giannelli, G., Falk-Marzillier, J., Schiraldi, O., Stetler-Stevenson, W.G., Quaranta, V., 1997. Induction of cell migration by matrix metalloprotease-2 cleavage of laminin-5. Science 277, 225-228.

Giannobile, W.V., 2008. Host-response therapeutics for periodontal diseases. J. Periodontol. 79, 1592-1600.

Gill, J.H., Kirwan, I.G., Seargent, J.M., Martin, S.W., Tijani, S., Anikin, V.A., Mearns, A.J., Bibby, M.C., Anthoney, A., Loadman, P.M., 2004. MMP-10 is overexpressed, proteolytically active, and a potential target for therapeutic intervention in human lung carcinomas. Neoplasia 6 , $777-785$.

Gill, S.E., Parks, W.C., 2008. Metalloproteinases and their inhibitors: regulators of wound healing. Int. J. Biochem. Cell Biol. $40,1334-1347$.

Gilles, C., Polette, M., Piette, J., Munaut, C., Thompson, E.W., Birembaut, P., Foidart, J.M., 1996. High level of MT-MMP expression is associated with invasiveness of cervical cancer cells. Int. J. Cancer 65, 209-213.

Gilles, C., Polette, M., Seiki, M., Birembaut, P., Thompson, E.W., 1997. Implication of collagen type I-induced membrane-type 1-matrix metalloproteinase expression and matrix metalloproteinase-2 activation in the metastatic progression of breast carcinoma. Lab. Invest. 76, 651-660.

Gilles, C., Polette, M., Coraux, C., Tournier, J.M., Meneguzzi, G., Munaut, C., Volders, L., Rousselle, P., Birembaut, P., Foidart, J.M., 2001. Contribution of MT1MMP and of human laminin-5 gamma2 chain degradation to mammary epithelial cell migration. J. Cell Sci. 114, 2967-2976.

Gingras, D., Bousquet-Gagnon, N., Langlois, S., Lachambre, M.P., Annabi, B., Beliveau, R., 2001. Activation of the extracellular signal regulated protein kinase (ERK) cascade by membrane-type-1 matrix metalloproteinase (MT1-MMP). FEBS Lett. 507, 231-236.

Gioia, M., Fasciglione, G.F., Marini, S., D’Alessio, S., De Sanctis, G., Diekmann, O., Pieper, M., Politi, V., Tschesche, H., Coletta, M., 2002. Modulation of the catalytic activity of neutrophil collagenase MMP-8 on bovine collagen I. Role of the activation cleavage and of the hemopexin-like domain. J. Biol. Chem. $277,23123-23130$

Gioia, M., Monaco, S., Fasciglione, G.F., Coletti, A., Modesti, A., Marini, S., Coletta, M., 2007. Characterization of the mechanisms by which gelatinase A, neutrophil collagenase, and membrane-type metalloproteinase MMP-14 recognize collagen I and enzymatically process the two alpha-chains. J. Mol. Biol. 368, 1101-1113.

Gioia, M., Monaco, S., Van Den Steen, P.E., Sbardella, D., Grasso, G., Marini, S., Overall, C.M., Opdenakker, G., Coletta, M., 2009. The collagen binding domain of gelatinase A modulates degradation of collagen IV by gelatinase B. J. Mol. Biol. 386, 419-434.

Gioia, M., Fasciglione, G.F., Monaco, S., Iundusi, R., Sbardella, D., Marini, S., Tarantino, U., Coletta, M., 2010. pH dependance of the enzymatic processing of collagen I by MMP-1 (fibroblast collagenase), MMP-2 (gelatinase A) and MMP-14 ectodomain. J. Biol. Inorg. Chem. 15, $1219-1232$.

Giricz, Z., Lalu, M.M., Csonka, C., Bencsik, P., Schulz, R., Ferdinandy, P., 2006. Hyperlipidemia attenuates the infarct size-limiting effect of ischemic preconditioning: role of matrix metalloproteinase-2 inhibition. J. Pharmacol. Exp. Ther. 316, 154-161.

Giricz, O., Lauer, J.L., Fields, G.B., 2010. Variability in melanoma metalloproteinase expression profiling. J. Biomol. Tech. 21, $194-204$.

Glass-Marmor, L., Paperna, T., Galboiz, Y., Miller, A., 2009. Immunomodulation by chronobiologically-based glucocorticoids treatment for multiple sclerosis relapses. J. Neuroimmunol. 210, 124-127.

Goldbach-Mansky, R., Lee, J.M., Hoxworth, J.M., Smith 2nd, D., Duray, P., Schumacher Jr., R.H., Yarboro, C.H., Klippel, J., Kleiner, D., El-Gabalawy, H.S., 2000. Active synovial matrix metalloproteinase-2 is associated with radiographic erosions in patients with early synovitis. Arthritis Res. 2, 145-153.

Gondi, C.S., Talluri, L., Dinh, D.H., Gujrati, M., Rao, J.S., 2009. RNAi-mediated downregulation of MMP-2 activates the extrinsic apoptotic pathway in human glioma xenograft cells. Int. J. Oncol. 35, 851-859.

Gottlieb, D.J., Stone, P.J., Sparrow, D., Gale, M.E., Weiss, S.T., Snider, G.L., O’Connor, G.T., 1996. Urinary desmosine excretion in smokers with and without rapid decline of lung function: the Normative Aging Study. Am. J. Respir. Crit. Care Med. 154, 1290-1295.

Gough, P.J., Gomez, I.G., Wille, P.T., Raines, E.W., 2006. Macrophage expression of active MMP-9 induces acute plaque disruption in apoE-deficient mice. J. Clin. Invest. 116, 59-69.

Gounni-Abdelilah, S., Wellemans, V., Agouli, M., Guenounou, M., Hamid, Q., Beck, L.A., Lamkhioued, B., 2006. Increased expression of Th2-associated chemokines in bullous pemphigoid disease. Role of eosinophils in the production and release of these chemokines. Clin. Immunol. 120, 220-231.

Gronski Jr., T.J., Martin, R.L., Kobayashi, D.K., Walsh, B.C., Holman, M.C., Huber, M., Van Wart, H.E., Shapiro, S.D., 1997. Hydrolysis of a broad spectrum of extracellular matrix proteins by human macrophage elastase. J. Biol. Chem. 272, 12189-12194.

Gruber, H.E., Ingram, J.A., Hoelscher, G.L., Zinchenko, N., Norton, H.J., Hanley Jr., E.N., 2009. Matrix metalloproteinase 28, a novel matrix metalloproteinase, is constitutively expressed in human intervertebral disc tissue and is present in matrix of more degenerated discs. Arthritis Res. Ther. 11 , R184.

Grzmil, P., Kim, Y., Shamsadin, R., Neesen, J., Adham, I.M., Heinlein, U.A., Schwarzer, U.J., Engel, W., 2001. Human cyritestin genes (CYRN1 and CYRN2) are non-functional. Biochem. J. 357, 551-556.

Gu, Z., Kaul, M., Yan, B., Kridel, S.J., Cui, J., Strongin, A., Smith, J.W., Liddington, R.C., Lipton, S.A., 2002. S-nitrosylation of matrix metalloproteinases: signaling pathway to neuronal cell death. Science 297, 1186-1190.

Guadamillas, M.C., Gonzalo, P., Hernandez-Riquer, M.V., Pollan, A., Grande-Garcia, A., Bartolomé, R.A., Vasanji, A., Ambrogio, C., Chiarle, R., Teixidó, J., Risteli, J., Apte, S.S., del Pozo, M.A., Arroyo, A.G., 2010. MT1-MMP is required for myeloid cell fusion via regulation of Rac1 signaling. Dev. Cell $18,77-89$.

Gueders, M.M., Balbin, M., Rocks, N., Foidart, J.M., Gosset, P., Louis, R., Shapiro, S., Lopez-Otin, C., Noël, A., Cataldo, D.D., 2005. Matrix metalloproteinase-8 deficiency promotes granulocytic allergen-induced airway inflammation. J. Immunol. 175, 2589-2597.

Guo, S., Wang, S., Kim, W.J., Lee, S.R., Frosch, M.P., Bacskai, B.J., Greenberg, S.M., Lo, E.H., 2006. Effects of apoE isoforms on beta-amyloid-induced matrix metalloproteinase-9 in rat astrocytes. Brain Res. 1111, 222-236.

Gutierrez-Fernandez, A., Inada, M., Balbin, M., Fueyo, A., Pitiot, A.S., Astudillo, A., Hirose, K., Hirata, M., Shapiro, S.D., Noël, A., Werb, Z., Krane, S.M., LopezOtin, C., Puente, X.S., 2007. Increased inflammation delays wound healing in mice deficient in collagenase-2 (MMP-8). FASEB J. 21, 2580-2591.

Ha, H.Y., Moon, H.B., Nam, M.S., Lee, J.W., Ryoo, Z.Y., Lee, T.H., Lee, K.K., So, B.J., Sato, H., Seiki, M., Yu, D.Y., 2001. Overexpression of membrane-type matrix metalloproteinase-1 gene induces mammary gland abnormalities and adenocarcinoma in transgenic mice. Cancer Res. 61, $984-990$.

Haas, T.L., Davis, S.J., Madri, J.A., 1998. Three-dimensional type I collagen lattices induce coordinate expression of matrix metalloproteinases MT1-MMP and MMP-2 in microvascular endothelial cells. J. Biol. Chem. 273, 3604-3610.

Habelhah, H., Okada, F., Kobayashi, M., Nakai, K., Choi, S., Hamada, J., Moriuchi, T., Kaya, M., Yoshida, K., Fujinaga, K., Hosokawa, M., 1999. Increased E1AF expression in mouse fibrosarcoma promotes metastasis through induction of MT1-MMP expression. Oncogene 18, $1771-1776$.

Hafezi-Moghadam, A., Ley, K., 1999. Relevance of L-selectin shedding for leukocyte rolling in vivo. J. Exp. Med. 189, 939-948.

Hägglund, A.C., Ny, A., Leonardsson, G., Ny, T., 1999. Regulation and localization of matrix metalloproteinases and tissue inhibitors of metalloproteinases in the mouse ovary during gonadotropin-induced ovulation. Endocrinology 140, 4351-4358.

Hahn, B.H., 1998. Antibodies to DNA. N. Engl. J. Med. 338, 1359-1368.

Hahn, B.H., 2003. Systemic lupus erythematosus and accelerated atherosclerosis. N. Engl. J. Med. 349, 2379-2380.

Hamann, G.F., Okada, Y., Fitridge, R., del Zoppo, G.J., 1995. Microvascular basal lamina antigens disappear during cerebral ischemia and reperfusion. Stroke $26,2120-2126$

Hanemaaijer, R., Sorsa, T., Konttinen, Y.T., Ding, Y., Sutinen, M., Visser, H., van Hinsbergh, V.W., Helaakoski, T., Kainulainen, T., Rönkä, H., Tschesche, H., Salo, T., 1997. Matrix metalloproteinase-8 is expressed in rheumatoid synovial fibroblasts and endothelial cells. Regulation by tumor necrosis factoralpha and doxycycline. J. Biol. Chem. 272, 31504-31509.

Hansson, G.K., 2005. Inflammation, atherosclerosis, and coronary artery disease. N. Engl. J. Med. 352, 1685-1695.

Hansson, G.K., Libby, P., 2006. The immune response in atherosclerosis: a double-edged sword. Nat. Rev. Immunol. 6, 508-519.

Hansson, J., Vasan, R.S., Ärnlöv, J., Ingelsson, E., Lind, L., Larsson, A., Michaëlsson, K., Sundström, J., 2011. Biomarkers of extracellular matrix metabolism (MMP-9 and TIMP-1) and risk of stroke, myocardial infarction, and cause-specific mortality: cohort study. PLoS One 6, e16185. 
Hao, L., Du, M., Lopez-Campistrous, A., Fernandez-Patron, C., 2004. Agonist-induced activation of matrix metalloproteinase-7 promotes vasoconstriction through the epidermal growth factor-receptor pathway. Circ. Res. 94, 68-76.

Haq, I., Chappell, S., Johnson, S.R., Lotya, J., Daly, L., Morgan, K., Guetta-Baranes, T., Roca, J., Rabinovich, R., Millar, A.B., Seamas, C.D., Keatings, V., MacNee, W., Stolk, J., Hiemstra, P.S., Miniati, M., Monti, S., O'Connor, C.M., Kalsheker, N., 2010. Association of MMP-12 polymorphisms with severe and very severe COPD: a case control study of MMPs-1, 9 and 12 in a European population. BMC Med. Genet. 11, 7.

Haq, I., Lowrey, G.E., Kalsheker, N., Johnson, S.R., in press. Matrix metalloproteinase-12 (MMP-12) SNP affects MMP activity, lung macrophage infiltration and protects against emphysema in COPD. Thorax.

Harrell, P.C., McCawley, L.J., Fingleton, B., McIntyre, J.O., Matrisian, L.M., 2005. Proliferative effects of apical, but not basal, matrix metalloproteinase-7 activity in polarized MDCK cells. Exp. Cell Res. 303, 308-320.

Harrison, R.K., Chang, B., Niedzwiecki, L., Stein, R.L., 1992. Mechanistic studies on the human matrix metalloproteinase stromelysin. Biochemistry 31, 10757-10762.

Harty, M.W., Huddleston, H.M., Papa, E.F., Puthawala, T., Tracy, A.P., Ramm, G.A., Gehring, S., Gregory, S.H., Tracy Jr., T.F., 2005. Repair after cholestatic liver injury correlates with neutrophil infiltration and matrix metalloproteinase 8 activity. Surgery 138, 313-320.

Hashimoto, G., Inoki, I., Fujii, Y., Aoki, T., Ikeda, E., Okada, Y., 2002. Matrix metalloproteinases cleave connective tissue growth factor and reactivate angiogenic activity of vascular endothelial growth factor 165. J. Biol. Chem. 277, 36288-36295.

Hashimoto, G., Shimoda, M., Okada, Y., 2004. ADAMTS4 (aggrecanase-1) interaction with the C-terminal domain of fibronectin inhibits proteolysis of aggrecan. J. Biol. Chem. 279, 32483-32491.

Hasty, K.A., Pourmotabbed, T.F., Goldberg, G.I., Thompson, J.P., Spinella, D.G., Stevens, R.M., Mainardi, C.L., 1990. Human neutrophil collagenase. A distinct gene product with homology to other matrix metalloproteinases. J. Biol. Chem. 265, 11421-11424.

Hattori, Y., Nerusu, K.C., Bhagavathula, N., Brennan, M., Hattori, N., Murphy, H.S., Su, L.D., Wang, T.S., Johnson, T.M., Varani, J., 2003. Vascular expression of matrix metalloproteinase-13 (collagenase-3) in basal cell carcinoma. Exp. Mol. Pathol. 74, 230-237.

Hattori, N., Mochizuki, S., Kishi, K., Nakajima, T., Takaishi, H., D’Armiento, J., Okada, Y., 2009. MMP-13 plays a role in keratinocyte migration, angiogenesis, and contraction in mouse skin wound healing. Am. J. Pathol. 175, 533-546.

Hauser, S., Bickel, L., Weinspach, D., Gerg, M., Schäfer, M.K., Pfeifer, M., Hazin, J., Schelter, F., Weidle, U.H., Ramser, J., Volkmann, J., Meindl, A., Schmitt, M., Schrötzlmair, F., Altevogt, P., Krüger, A., 2011. Full-length L1CAM and not its $\Delta 2 \Delta 27$ splice variant promotes metastasis through induction of gelatinase expression. PLoS One 6, e18989.

Hautamaki, R.D., Kobayashi, D.K., Senior, R.M., Shapiro, S.D., 1997. Requirement for macrophage elastase for cigarette smoke-induced emphysema in mice. Science 277, 2002-2004.

Hayashita-Kinoh, H., Kinoh, H., Okada, A., Komori, K., Itoh, Y., Chiba, T., Kajita, M., Yana, I., Seiki, M., 2001. Membrane-type 5 matrix metalloproteinase is expressed in differentiated neurons and regulates axonal growth. Cell Growth Differ. 12, 573-580.

Hawinkels, L.J., Kuiper, P., Wiercinska, E., Verspaget, H.W., Liu, Z., Pardali, E., Sier, C.F., ten Dijke, P., 2010. Matrix metalloproteinase-14 (MT1-MMP)mediated endoglin shedding inhibits tumor angiogenesis. Cancer Res. 70, 4141-4150.

Hawkins, B.T., Davis, T.P., 2005. The blood-brain barrier/neurovascular unit in health and disease. Pharmacol. Rev. 57, $173-185$.

Hegedüs, L., Cho, H., Xie, X., Eliceiri, G.L., 2008. Additional MDA-MB-231 breast cancer cell matrix metalloproteinases promote invasiveness. J. Cell. Physiol. $216,480-485$.

Heiskanen, T.J., Illman, S.A., Lohi, J., Keski-Oja, J., 2009. Epilysin (MMP-28) is deposited to the basolateral extracellular matrix of epithelial cells. Matrix Biol. 28, 74-83.

Heljasvaara, R., Nyberg, P., Luostarinen, J., Parikka, M., Heikkila, P., Rehn, M., Sorsa, T., Salo, T., Pihlajaniemi, T., 2005. Generation of biologically active endostatin fragments from human collagen XVIII by distinct matrix metalloproteases. Exp. Cell Res. 307, $292-304$.

Henderson, B.C., Tyagi, S.C., 2006. Oxidative mechanism and homeostasis of proteinase/antiproteinase in congestive heart failure. J. Mol. Cell. Cardiol. 41, 959-962.

Henderson, B.C., Tyagi, N., Ovechkin, A., Kartha, G.K., Moshal, K.S., Tyagi, S.C., 2007a. Oxidative remodeling in pressure overload induced chronic heart failure. Eur. J. Heart Fail. 9, 450-457.

Henderson, B.C., Sen, U., Reynolds, C., Moshal, K.S., Ovechkin, A., Tyagi, N., Kartha, G.K., Rodriguez, W.E., Tyagi, S.C., 2007b. Reversal of systemic hypertension-associated cardiac remodeling in chronic pressure overload myocardium by ciglitazone. Int. J. Biol. Sci. 3, 385-392.

Henney, A.M., Wakeley, P.R., Davies, M.J., Foster, K., Hembry, R., Murphy, G., Humphries, S., 1991. Localization of stromelysin gene expression in atherosclerotic plaques by in situ hybridization. Proc. Natl. Acad. Sci. USA 88, 8154-8158.

Heo, J.H., Lucero, J., Abumiya, T., Koziol, J.A., Copeland, B.R., del Zoppo, G.J., 1999. Matrix metalloproteinases increase very early during experimental focal cerebral ischemia. J. Cereb. Blood Flow Metab. 19, 624-633.

Heo, S.H., Cho, C.H., Kim, H.O., Jo, Y.H., Yoon, K.S., Lee, J.H., Park, J.C., Park, K.C., Ahn, T.B., Chung, K.C., Yoon, S.S., Chang, D.I., 2011. Plaque rupture is a determinant of vascular events in carotid artery atherosclerotic disease: involvement of matrix metalloproteinases 2 and 9. J. Clin. Neurol. 7, 69-76.

Herman, M.P., Sukhova, G.K., Libby, P., Gerdes, N., Tang, N., Horton, D.B., Kilbride, M., Breitbart, R.E., Chun, M., Schönbeck, U., 2001. Expression of neutrophil collagenase (matrix metalloproteinase-8) in human atheroma: a novel collagenolytic pathway suggested by transcriptional profiling. Circulation 104, 1899-1904.

Hernandez-Guillamon, M., Delgado, P., Ortega, L., Pares, M., Rosell, A., Garcia-Bonilla, L., Fernandez-Cadenas, I., Borrell-Pages, M., Boada, M., Montaner, J., 2009. Neuronal TIMP-1 release accompanies astrocytic MMP-9 secretion and enhances astrocyte proliferation induced by beta-amyloid 25-35 fragment. J. Neurosci. Res. 87, 21115-21125.

Hernandez-Guillamon, M., Mawhirt, S., Fossati, S., Blais, S., Pares, M., Penalba, A., Boada, M., Couraud, P.O., Neubert, T.A., Montaner, J., Ghiso, J., Rostagno, A., 2010. Matrix metalloproteinase 2 (MMP-2) degrades soluble vasculotropic amyloid-beta E22Q and L34V mutants, delaying their toxicity for human brain microvascular endothelial cells. J. Biol. Chem. 285, 27144-27158.

Herrmann, J.L., Abarbanell, A.M., Wang, Y., Weil, B.R., Poynter, J.A., Manukyan, M.C., Meldrum, D.R., 2011. Transforming growth factor- $\alpha$ enhances stem cellmediated postischemic myocardial protection. Ann. Thorac. Surg. 92, 1719-1725.

Hersh, L.B., Rodgers, D.W., 2008. Neprilysin and amyloid beta peptide degradation. Curr. Alzheimer Res. 5, $225-231$.

Heymans, S., Luttun, A., Nuyens, D., Theilmeier, G., Creemers, E., Moons, L., Dyspersin, G.D., Cleutjens, J.P., Shipley, M., Angellilo, A., Levi, M., Nübe, O., Baker, A., Keshet, E., Lupu, F., Herbert, J.M., Smits, J.F., Shapiro, S.D., Baes, M., Borgers, M., Collen, D., Daemen, M.J., Carmeliet, P., 1999. Inhibition of plasminogen activators or matrix metalloproteinases prevents cardiac rupture but impairs therapeutic angiogenesis and causes cardiac failure. Nat. Med. 5, 11351142.

Heymans, S., Lupu, F., Terclavers, S., Vanwetswinkel, B., Herbert, J.M., Baker, A., Collen, D., Carmeliet, P., Moons, L., 2005. Loss or inhibition of uPA or MMP-9 attenuates LV remodeling and dysfunction after acute pressure overload in mice. Am. J. Pathol. 166, 15-25.

Hiden, U., Wadsack, C., Prutsch, N., Gauster, M., Weiss, U., Frank, H.G., Schmitz, U., Fast-Hirsch, C., Hengstschläger, M., Pötgens, A., Rüben, A., Knöfler, M., Haslinger, P., Huppertz, B., Bilban, M., Kaufmann, P., Desoye, G., 2007. The first trimester human trophoblast cell line ACH-3P: a novel tool to study autocrine/paracrine regulatory loops of human trophoblast subpopulations - TNF-alpha stimulates MMP-15 expression. BMC Dev. Biol. 7, 137.

Hieta, N., Impola, U., Lopez-Otin, C., Saarialho-Kere, U., Kähäri, V.M., 2003. Matrix metalloproteinase-19 expression in dermal wounds and by fibroblasts in culture. J. Invest. Dermatol. 121, 997-1004

Hiller, O., Lichte, A., Oberpichler, A., Kocourek, A., Tschesche, H., 2000. Matrix metalloproteinases collagenase-2, macrophage elastase, collagenase-3, and membrane type 1-matrix metalloproteinase impair clotting by degradation of fibrinogen and factor XII. J. Biol. Chem. 275, 33008-33013.

Himelstein, B.P., Canete-Soler, R., Bernhard, E.J., Muschel, R.J., 1994. Induction of fibroblast 92 kDa gelatinase/type IV collagenase expression by direct contact with metastatic tumor cells. J. Cell Sci. 107, 477-486.

Hiraoka, M., Furukawa, T., 1998. Functional modulation of cardiac ATP-sensitive K(+) channels. News Physiol. Sci. 13, 131-137. 
Hiratsuka, S., Watanabe, A., Aburatani, H., Maru, Y., 2006. Tumour-mediated upregulation of chemoattractants and recruitment of myeloid cells predetermines lung metastasis. Nat. Cell Biol. 8, 1369-1375.

Hirose, T., Riefe, R.A., Smith Jr., G.N., Stevens, R.M., Mainardi, C.L., Hasty, K.A., 1992. Characterization of type V collagenase (gelatinase) in synovial fluid of patients with inflammatory arthritis. J. Rheumatol. 19, 593-599.

Hirose, T., Patterson, C., Pourmotabbed, T., Mainardi, C.L., Hasty, K.A., 1993. Structure-function relationship of human neutrophil collagenase: identification of regions responsible for substrate specificity and general proteinase activity. Proc. Natl. Acad. Sci. USA 90, $2569-2573$.

Hofmann, H.S., Hansen, G., Richter, G., Taege, C., Simm, A., Silber, R.E., Burdach, S., 2005. Matrix metalloproteinase-12 expression correlates with local recurrence and metastatic disease in non-small cell lung cancer patients. Clin. Cancer Res. 11, 1086-1092.

Hohlbaum, A.M., Moe, S., Marshak-Rothstein, A., 2000. Opposing effects of transmembrane and soluble Fas ligand expression on inflammation and tumor cell survival. J. Exp. Med. 191, 1209-1220.

Hojilla, C.V., Mohammed, F.F., Khokha, R., 2003. Br. J. Cancer 89, 1817-1821.

Holman, C.M., Kan, C.-C., Gehring, M.R., van Wart, H.E., 1999. Role of His-224 in the anomalous pH dependence of human stromelysin-1. Biochemistry 38, 677-681.

Holmbeck, K., Bianco, P., Caterina, J., Yamada, S., Kromer, M., Kuznesov, S.A., Mankani, M., Robey, P.G., Poole, A.R., Pidoux, I., Ward, J.M., Birkdal-Hansen, H., 1999. MT1-MMP-deficient mice develop dwarfism, osteopenia, arthritis and connective tissue disease due to inadequate collagen turnover. Cell 99, $81-$ 92.

Hopkins, D.R., Keles, S., Greenspan, D.S., 2007. The bone morphogenetic protein 1/Tolloid-like metalloproteinases. Matrix Biol. 26, 508-523.

Horton, M.R., Shapiro, S., Bao, C., Lowenstein, C.J., Noble, P.W., 1999. Induction and regulation of macrophage metalloelastase by hyaluronan fragments in mouse macrophages. J. Immunol. 162, 4171-4176.

Hoshino, M., Nakamura, Y., Sim, J., Shimojo, J., Isogai, S., 1998. Bronchial subepithelial fibrosis and expression of matrix metalloproteinase-9 in asthmatic airway inflammation. J. Allergy Clin. Immunol. 102, 783-788.

Hotary, K., Allen, E., Punturieri, A., Yana, I., Weiss, S.J., 2000. Regulation of cell invasion and morphogenesis in a three-dimensional type I collagen matrix by membrane-type matrix metalloproteinases 1, 2, and 3. J. Cell Biol. 149, 1309-1323.

Hotary, K.B., Allen, E.D., Brooks, P.C., Datta, N.S., Long, M.W., Weiss, S.J., 2003. Membrane type I matrix metalloproteinase usurps tumor growth control imposed by the three-dimensional extracellular matrix. Cell 114, 33-45.

Houghton, A.M., Quintero, P.A., Perkins, D.L., Kobayashi, D.K., Kelley, D.G., Marconcini, L.A., Mecham, R.P., Senior, R.M., Shapiro, S.D., 2006. Elastin fragments drive disease progression in a murine model of emphysema. J. Clin. Invest. 116, 753-759.

Howard, L., Maciewicz, R.A., Blobel, C.P., 2000. Cloning and characterization of ADAM28: evidence for autocatalytic pro-domain removal and for cell surface localization of mature ADAM28. Biochem. J. 348, 21-27.

Hu, J., Van den Steen, P.E., Sang, Q.-X.A., Opdenakker, G., 2007. Matrix metalloproteinase inhibitors as therapy for inflammatory and vascular diseases. Nat. Rev. Drug Discov. 6, 480-498.

Hu, Q., Chen, C., Khatibi, N.H., Li, L., Yang, L., Wang, K., Han, J., Duan, W., Zhang, J.H., Zhou, C., 2010. Lentivirus-mediated transfer of MMP-9 shRNA provides neuroprotection following focal ischemic brain injury in rats. Brain Res. 1367, 347-359.

Huang, Y., Li, W., Chu, D., Zheng, J., Ji, G., Li, M., Zhang, H., Wang, W., Du, J., Li, J., 2011. Overexpression of matrix metalloproteinase-21 is associated with poor overall survival of patients with colorectal cancer. J. Gastrointest. Surg. 15, 1188-1194.

Hulkkonen, J., Pertovaara, M., Antonen, J., Pasternack, A., Hurme, M., Pöllänen, P., Lehtimäki, T., 2004. Matrix metalloproteinase 9 (MMP-9) gene polymorphism and MMP-9 plasma levels in primary Sjogren's syndrome. Rheumatology (Oxford) 43, 1476-1479.

Hunninghake, G.M., Cho, M.H., Tesfaigzi, Y., Soto-Quiros, M.E., Avila, L., Lasky-Su, J., Stidley, C., Melen, E., Söderhäll, C., Hallberg, J., Kull, I., Kere, J., Svartengren, M., Pershagen, G., Wickman, M., Lange, C., Demeo, D.L., Hersh, C.P., Klanderman, B.J., Raby, B.A., Sparrow, D., Shapiro, S.D., Silverman, E.K. Litonjua, A.A., Weiss, S.T., Celedon, J.C., 2009. MMP12, lung function, and COPD in high-risk populations. N. Engl. J. Med. 361, 2599-2608.

Huo, N., Ichikawa, Y., Kamiyama, M., Ishikawa, T., Hamaguchi, Y., Hasegawa, S., Nagashima, Y., Miyazaki, K., Shimada, H., 2002. MMP-7 (matrilysin) accelerated growth of human umbilical vein endothelial cells. Cancer Lett. 177, 95-100.

Hwang, L., Leichter, R., Okamoto, A., Payan, D., Collins, S.M., Bunnett, N.W., 1993. Downregulation of neutral endopeptidase (EC 3.4.24.11) in the inflamed rat intestine. Am. J. Physiol. 264, G735-G743.

Ihling, C., Szombathy, T., Bohrmann, B., Brockhaus, M., Schaefer, H.E., Loezer, B.M., 2001. Coexpression of endothelin-converting enzyme-1 and endothelin-1 in diverse stages of human atherosclerosis. Circulation 104, 864-869.

Ihling, C., Bohrmann, B., Schaefer, H.E., Technau-Ihling, K., Loezer, B.M., 2004. Endothelin-1 and endothelin converting enzyme-1 in human atherosclerosisnovel targets for pharmacotherapy in atherosclerosis. Curr. Vasc. Pharmacol. 2, 249-258.

Ii, M., Yamamoto, H., Adachi, Y., Maruyama, Y., Shinomura, Y., 2006. Role of matrix metalloproteinase-7 (matrilysin) in human cancer invasion, apoptosis growth, and angiogenesis. Exp. Biol. Med. 231, 20-27.

Iida, J., Wilhelmson, K.L., Price, M.A., Wilson, C.M., Pei, D., Furcht, L.T., McCarthy, J.B., 2004. Membrane type-1 matrix metalloproteinase promotes human melanoma invasion and growth. J. Invest. Dermatol. 122, 167-176.

Iimuro, Y., Nishio, T., Morimoto, T., Nitta, T., Stefanovic, B., Choi, S.K., Brenner, D.A., Yamaoka, Y., 2003. Delivery of matrix metalloproteinase-1 attenuates established liver fibrosis in the rat. Gastroenterology 124, 445-458.

Ikari, A., Sato, T., Takiguchi, A., Atomi, K., Yamazaki, Y., Sugatani, J., 2011. Claudin-2 knockdown decreases matrix metalloproteinase-9 activity and cell migration via suppression of nuclear Sp1 in A549 cells. Life Sci. 88, 628-633.

Illman, S.A., Keski-Oja, J., Lohi, J., 2001. Promoter characterization of the human and mouse epilysin (MMP-28) genes. Gene 275, 185-194.

Illman, S.A., Lehti, K., Keski-Oja, J., Lohi, J., 2006. Epilysin (MMP-28) induces TGF-beta mediated epithelial to mesenchymal transition in lung carcinoma cells. J. Cell Sci. 119, 3856-3865.

Illman, S.A., Lohi, J., Keski-Oja, J., 2008. Epilysin (MMP-28) - structure, expression and potential functions. Exp. Dermatol. 17, 897-907.

Imai, K., Yokohama, Y., Nakanishi, I., Ohuchi, E., Fujii, Y., Nakai, N., Okada, Y., 1995. Matrix metalloproteinase 7 (matrilysin) from human rectal carcinoma cells. Activation of the precursor, interaction with other matrix metalloproteinases and enzymic properties. J. Biol. Chem. 270 , 6691-6697.

Imai, K., Hiramatsu, A., Fukushima, D., Pierschbacher, M.D., Okada, Y., 1997. Degradation of decorin by matrix metalloproteinases: identification of the cleavage sites, kinetic analyses and transforming growth factor-beta1 release. Biochem. J. 322, 809-814.

Imai, K., Dalal, S.S., Chen, E.S., Downey, R., Schulman, L.L., Ginsburg, M., D’Armiento, J., 2001. Human collagenase (matrix metalloproteinase-1) expression in the lungs of patients with emphysema. Am. J. Respir. Crit. Care Med. 163, 786-791.

Impola, U., Toriseva, M., Suomela, S., Jeskanen, L., Hieta, N., Jahkola, T., Grenman, R., Kähäri, V.M., Saarialho-Kere, U., 2003. Matrix metalloproteinase-19 is expressed by proliferating epithelium but disappears with neoplastic dedifferentiation. Int. J. Cancer 103, $709-716$.

Impola, U., Jeskanen, L., Ravanti, L., Syrjanen, S., Baldursson, B., Kähäri, V.M., Saarialho-Kere, U., 2005. Expression of matrix metalloproteinase MMP-7 and MMP-13 and loss of MMP-19 and p16 are associated with malignant progression in chronic wounds. Br. J. Dermatol. 152, 720-726.

Inoue, A., Takahashi, H., Harada, H., Kohno, S., Ohue, S., Kobayashi, K., Yano, H., Tanaka, J., Ohnishi, T., 2010. Cancer stem-like cells of glioblastoma characteristically express MMP-13 and display highly invasive activity. Int. J. Oncol. 37, 1121-1131.

Iredale, J.P., Benyon, R.C., Pickering, J., McCullen, M., Northrop, M., Pawley, S., Hovell, C., Arthur, M.J., 1998. Mechanisms of spontaneous resolution of rat liver fibrosis. Hepatic stellate cell apoptosis and reduced hepatic expression of metalloproteinase inhibitors. J. Clin. Invest. 102, 538-549.

Ito, K., Funayama, S., Hitomi, Y., Nomura, S., Katsura, K., Saito, M., Hayashi, T., Kaneko, N., Nohno, K., Igarashi, A., 2009. Proteome analysis of gelatin-bound salivary proteins in patients with primary Sjögren's syndrome: identification of matrix metalloproteinase-9. Clin. Chim. Acta 403, 269-271.

Itoh, T., Tanioka, M., Yoshida, H., Yoshioka, T., Nishimoto, H., Itohara, S., 1998. Reduced angiogenesis and tumor progression in gelatinase A-deficient mice. Cancer Res. 58, 1048-1051. 
Itoh, T., Tanioka, M., Matsuda, H., Nishimoto, H., Yoshioka, T., Suzuki, R., Uehira, M., 1999. Experimental metastasis is suppressed in MMP-9-deficient mice. Clin. Exp. Metastasis 17, 177-181.

Itoh, Y., Takamura, A., Ito, N., Maru, Y., Sato, H., Suenaga, N., Aoki, T., Seiki, M., 2001. Homophilic complex formation of MT1-MMP facilitates proMMP-2 activation on the cell surface and promotes tumor cell invasion. EMBO J. 20, 4782-4793.

Itoh, T., Matsuda, H., Tanioka, M., Kuwabara, K., Itohara, S., Suzuki, R., 2002. The role of matrix metalloproteinase-2 and matrix metalloproteinase-9 in antibody-induced arthritis. J. Immunol. 169, 2643-2647.

Itoh, Y., Seiki, M., 2006. MT1-MMP: a potent modifier of pericellular microenvironment. J. Cell. Physiol. 206, 1-8.

Iwata, N., Tsubuki, S., Takaki, Y., Watanabe, K., Seikiguchi, M., Hosoki, E., Kawashima-Morishima, M., Lee, H.-J., Hama, E., Sekine-Aizawa, Y., Saido, T.C., 2000. Identification of the major A_1-42-degrading catabolic pathway in brain parenchyma: suppression leads to biochemical and pathological deposition. Nat. Med. 6, 143-150.

Janssens, S., Lijnen, H.R., 2006. What has been learned about the cardiovascular effects of matrix metalloproteinases from mouse models? Cardiovasc. Res. 69, 585-594.

Jaworski, D.M., 2000. Developmental regulation of membrane type-5 matrix metalloproteinase (MT5-MMP) expression in the rat nervous system. Brain Res. 860, 174-177.

Jefferis, B.J., Whincup, P., Welsh, P., Wannamethee, G., Rumley, A., Lennon, L., Thomson, A., Lawlor, D., Carson, C., Ebrahim, S., Lowe, G., 2010. Prospective study of matrix metalloproteinase-9 and risk of myocardial infarction and stroke in older men and women. Atherosclerosis 208, 557-563.

Jeffries, M.A., Dozmorov, M., Tang, Y., Merrill, J.T., Wren, J.D., Sawalha, A.H., 2011. Genome-wide DNA methylation patterns in CD4+ T cells from patients with systemic lupus erythematosus. Epigenetics 6, 593-601.

Jeng, A.Y., Chou, M., Sawyer, W.K., Caplan, S.L., Von Linden-Reed, J., Jeune, M., Prescott, M.F., 1999. Enhanced expression of matrix metalloproteinase-3, -12, and -13 mRNAs in the aortas of apolipoprotein E-deficient mice with advanced atherosclerosis. Ann. NY Acad. Sci. 878, 555-558.

Jensen, S.A., Vainer, B., Bartels, A., Brünner, N., Sørensen, J.B., 2010. Expression of matrix metalloproteinase 9 (MMP-9) and tissue inhibitor of metalloproteinases 1 (TIMP-1) by colorectal cancer cells and adjacent stroma cells-associations with histopathology and patients outcome. Eur. J. Cancer 46, 3233-3242.

Jia, J., Kang, S., Zhao, J., Zhang, X., Wang, N., Zhou, R., Li, Y., 2010. Association of functional polymorphisms on MMP-12 and MMP-13 gene promoter region with epithelial ovarian carcinoma. Zhonghua Yi Xue Yi Chuan Xue Za Zhi 27, 209-213.

Jian, P., Yanfang, T., Zhuan, Z., Jian, W., Xueming, Z., Jian, N., 2011. MMP28 (epilysin) as a novel promoter of invasion and metastasis in gastric cancer. BMC Cancer 11, 200.

Jin, X., Yagi, M., Akiyama, N., Hirosaki, T., Higashi, S., Lin, C.Y., Dickson, R.B., Kitamura, H., Miyazaki, K., 2006. Matriptase activates stromelysin (MMP-3) and promotes tumor growth and angiogenesis. Cancer Sci. 97, 1327-1334.

Jo, Y.K., Park, S.J., Shin, J.H., Kim, Y., Hwang, J.J., Cho, D.H., Kim, J.C., 2011. ARP101, a selective MMP-2 inhibitor, induces autophagy-associated cell death in cancer cells. Biochem. Biophys. Res. Commun. 404, 1039-1043.

Joergensen, M.T., Brünner, N., De Muckadell, O.B., 2010. Comparison of circulating MMP-9, TIMP-1 and CA19-9 in the detection of pancreatic cancer. Anticancer Res. 30, 587-592.

Johansson, N., Saarialho-Kere, U., Airola, K., Herva, R., Nissinen, L., Westermarck, J., Vuorio, E., Heino, J., Kähäri, V.M., 1997a. Collagenase-3 (MMP-13) is expressed by hypertrophic chondrocytes, periosteal cells, and osteoblasts during human fetal bone development. Dev. Dyn. 208, 387-397.

Johansson, N., Airola, K., Grenman, R., Kariniemi, A.L., Saarialho-Kere, U., Kähäri, V.M., 1997b. Expression of collagenase-3 (matrix metalloproteinase-13) in squamous cell carcinomas of the head and neck. Am. J. Pathol. 151, 499-508.

Johansson, N., Vaalamo, M., Grenman, S., Hietanen, S., Klemi, P., Saarialho-Kere, U., Kähäri, V.M., 1999. Collagenase-3 (MMP-13) is expressed by tumor cells in invasive vulvar squamous cell carcinomas. Am. J. Pathol. 154, 469-480.

Johnson, L.L., Pavlovsky, A.G., Johnson, A.R., Janowicz, J.A., Man, C.-F., Ortwine, D.F., Purchase II, C.F., White, A.D., 2000. A rationalization of the acidic pH dependence for stromelysin-1 (matrix metalloproteinase-3) catalysis and inhibition. J. Biol. Chem. 275, 11026-11033.

Johnson, J.L., George, S.J., Newby, A.C., Jackson, C.L., 2005. Divergent effects of matrix metalloproteinases 3, 7, 9, and 12 on atherosclerotic plaque stability in mouse brachiocephalic arteries. Proc. Natl. Acad. Sci. USA 102, 15575-15580.

Johnson, A.R., Pavlovsky, A.G., Ortwine, D.F., Prior, F., Man, C.F., Bornemeier, D.A., Banotai, C.A., Mueller, W.T., McConnell, P., Yan, C., Baragi, V., Lesch, C., Roark, W.H., Wilson, M., Datta, K., Guzman, R., Han, H.K., Dyer, R.D., 2007. Discovery and characterization of a novel inhibitor of matrix metalloprotease13 that reduces cartilage damage in vivo without joint fibroplasia side effects. J. Biol. Chem. 282, 27781-27791.

Johnson, J.L., Newby, A.C., Jackson, C.L., Devel, L., Czarny, B., Rogakos, V., Beau, F., Yiotakis, A., Dive, V., 2008. Inhibition of MMP-12 retards atherosclerotic plaque development and increases stability in the apolipoprotein E-deficient mouse. Circulation 118, S483.

Johnston, P., Chojnowski, A.J., Davidson, R.K., Riley, G.P., Donell, S.T., Clark, I.M., 2007. A complete expression profile of matrix-degrading metalloproteinases in Dupuytren's disease. J. Hand Surg. Am. 32, 343-351.

Jones, L.E., Humphreys, M.J., Campbell, F., Neoptolemos, J.P., Boyd, M.T., 2004. Comprehensive analysis of matrix metalloproteinase and tissue inhibitor expression in pancreatic cancer: increased expression of matrix metalloproteinase-7 predicts poor survival. Clin. Cancer Res. $10,2832-2845$.

Jones, G.C., Riley, G.P., 2005. ADAMTS proteinases: a multi-domain, multi-functional family with roles in extracellular matrix turnover and arthritis. Arthritis Res. Ther. 7, 160-169.

Jormsjo, S., Ye, S., Moritz, J., Walter, D.H., Dimmeler, S., Zeiher, A.M., Henney, A., Hamsten, A., Eriksson, P., 2000. Allele-specific regulation of matrix metalloproteinase-12 gene activity is associated with coronary artery luminal dimensions in diabetic patients with manifest coronary artery disease. Circ. Res. 86, 998-1003.

Jonsson-Rylander, A.C., Nilsson, T., Fritsche-Danielson, R., Hammarstrom, A., Behrendt, M., Andersson, J.O., Lindgren, K., Andersson, A.K., Wallbrandt, P., Rosengren, B., Brodin, P., Thelin, A., Westin, A., Hurt-Camejo, E., Lee-Søgaard, C.H., 2005. Role of ADAMTS-1 in atherosclerosis: remodeling of carotid artery, immunohistochemistry, and proteolysis of versican. Arterioscler Thromb. Vasc. Biol. 25, 180-185.

Jost, M., Folgueras, A.R., Frerart, F., Pendas, A.M., Blacher, S., Houard, X., Berndt, S., Munaut, C., Cataldo, D., Alvarez, J., Melen-Lamalle, L., Foidart, J.M., LopezOtin, C., Noël, A., 2006. Earlier onset of tumoral angiogenesis in matrix metalloproteinase-19-deficient mice. Cancer Res. 66, 5234-5241.

Juncker-Jensen, A., Lund, L.R., 2011. Phenotypic overlap between MMP-13 and the plasminogen activation system during wound healing in mice. PLoS One 6, e16954.

Jung, M., Römer, A., Keyszer, G., Lein, M., Kristiansen, G., Schnorr, D., Loening, S.A., Jung, K., 2003. mRNA expression of the five membrane-type matrix metalloproteinases MT1-MT5 in human prostatic cell lines and their down-regulation in human malignant prostatic tissue. Prostate 55, 89-98.

Jung, H.O., Uhm, J.S., Seo, S.M., Kim, J.H., Youn, H.J., Baek, S.H., Chung, W.S., Seung, K.B., 2010. Angiotensin II-induced smooth muscle cell migration is mediated by LDL receptor-related protein 1 via regulation of matrix metalloproteinase 2 expression. Biochem. Biophys. Res. Commun. 402, 577-582.

Jüngel, A., Ospelt, C., Lesch, M., Thiel, M., Sunyer, T., Schorr, O., Michel, B.A., Gay, R.E., Kolling, C., Flory, C., Gay, S., Neidhart, M., 2010. Effect of the oral application of a highly selective MMP-13 inhibitor in three different animal models of rheumatoid arthritis. Ann. Rheum Dis. 69, $898-902$.

Jury, J.A., Frayne, J., Hall, L., 1997. The human fertilin alpha gene is non-functional: implications for its proposed role in fertilization. Biochem. J. 321, 577581.

Kadono, Y., Shibahara, K., Namiki, M., Watanabe, Y., Seiki, M., Sato, H., 1998. Membrane type 1-matrix metalloproteinase is involved in the formation of hepatocyte growth factor/scatter factor-induced branching tubules in madin-darby canine kidney epithelial cells. Biochem. Biophys. Res. Commun. 251 , 681-687.

Kadonosono, T., Kato, M., Ueda, M., 2007. Metallopeptidase neurolysin as a novel molecular tool for analysis of properties of cancer-producing matrix metalloproteinases-2 and -9. Appl. Microbiol. Biotechnol. 75, 1285-1291.

Kajita, M., Kinoh, H., Ito, N., Takamura, A., Itoh, Y., Okada, A., Sato, H., Seiki, M., 1999. Human membrane type-4 matrix metalloproteinase (MT4-MMP) is encoded by a novel major transcript: isolation of complementary DNA clones for human and mouse mt4-mmp transcripts. FEBS Lett. 457, 353-356. 
Kajita, M., Itoh, Y., Chiba, T., Mori, H., Okada, A., Kinoh, H., Seiki, M., 2001. Membrane-type 1 matrix metalloproteinase cleaves CD44 and promotes cell migration. J. Cell Biol. 153, 893-904.

Kaminska, M., Foley, S., Maghni, K., Storness-Bliss, C., Coxson, H., Ghezzo, H., Lemière, C., Olivenstein, R., Ernst, P., Hamid, Q., Martin, J., 2009. Airway remodeling in subjects with severe asthma with or without chronic persistent airflow obstruction. J. Allergy Clin. Immunol. 124 , $45-51$.

Kanayama, H., Yokota, K., Kurokawa, Y., Murakami, Y., Nishitani, M., Kagawa, S., 1998. Prognostic values of matrix metalloproteinase-2 and tissue inhibitor of metalloproteinase-2 expression in bladder cancer. Cancer 82, 1359-1366.

Kaneko, H., Anzai, T., Horiuchi, K., Kohno, T., Nagai, T., Anzai, A., Takahashi, T., Sasaki, A., Shimoda, M., Maekawa, Y., Shimizu, H., Yoshikawa, T., Okada, Y., Yozu, R., Fukuda, K., 2011. Tumor necrosis factor- $\alpha$ converting enzyme is a key mediator of abdominal aortic aneurysm development. Atherosclerosis 218, 470-478.

Kanemitsu, H., Tomiyama, T., Mori, H., 2003. Human neprilysin is capable of degrading amyloid beta peptide not only in the monomeric form but also the pathological oligomeric form. Neurosci. Lett. 350, 113-116.

Kang, T., Yi, J., Guo, A., Wang, X., Overall, C.M., Jiang, W., Elde, R., Borregaard, N., Pei, D., 2001. Subcellular distribution and cytokine- and chemokineregulated secretion of leukolysin/MT6-MMP/MMP-25 in neutrophils. J. Biol. Chem. 276, 21960-21968.

Kang, H.R., Cho, S.J., Lee, C.G., Homer, R.J., Elias, J.A., 2007. Transforming growth factor (TGF)- $\beta$ stimulates pulmonary fibrosis and inflammation via a Baxdependent, bid-activated pathway that involves matrix metalloproteinase-12. J. Biol. Chem. 282, 7723-7732.

Kang, M.J., Jung, S.A., Jung, J.M., Kim, S.E., Jung, H.K., Kim, T.H., Shim, K.N., Yi, S.Y., Yoo, K., Moon, I.H., 2011. Associations between single nucleotide polymorphisms of MMP2, VEGF, and HIF1A genes and the risk of developing colorectal cancer. Anticancer Res. 31, 575-584.

Kaplan, R.N., Rafii, S., Lyden, D., 2006. Preparing the soil: the premetastatic niche. Cancer Res. 66, 11089-11093.

Kaplan, R.N., Riba, R.D., Zacharoulis, S., Bramley, A.H., Vincent, L., Costa, C., MacDonald, D.D., Jin, D.K., Shido, K., Kerns, S.A., Zhu, Z., Hicklin, D., Wu, Y., Port, J.L., Altorki, N., Port, E.R., Ruggero, D., Shmelkov, S.V., Jensen, K.K., Rafii, S., Lyden, D., 2005. VEGFR1-positive haematopoietic bone marrow progenitors initiate the pre-metastatic niche. Nature 438, 820-827.

Karlberg, T., van den Berg, S., Hammarström, M., Sagemark, J., Johansson, I., Holmberg-Schiavone, L., Schüler, H., 2009. Crystal structure of the ATPase domain of the human AAA+ protein paraplegin/SPG7. PLoS One 4, e6975.

Karroum, A., Mirshahi, P., Benabbou, N., Faussat, A.M., Soria, J., Therwath, A., Mirshahi, M., Hatmi, M., 2010. Matrix metalloproteinase-9 is required for tubular network formation and migration of resistant breast cancer cells MCF-7 through PKC and ERK1/2 signalling pathways. Cancer Lett. 295, 242251.

Kasperkiewicz, M., Zillikens, D., Schmidt, E., in press. Pemphigoid diseases: pathogenesis, diagnosis, and treatment. Autoimmunity.

Kato, R., Kiryu-Seo, S., Kiyama, H., 2002. Damage-induced neuronal endopeptidase (DINE/ECEL) expression is regulated by leukemia inhibitory factor and deprivation of nerve growth factor in rat sensory ganglia after nerve injury. J. Neurosci. 22, 9410-9418.

Katzenstein, A.L.A., Myers, J.L., 1998. Idiopathic pulmonary fibrosis - clinical relevance of pathologic classification. Am. J. Respir. Crit. Care Med. 157, 13011315.

Kay, J.N., De la Huerta, I., Kim, I.J., Zhang, Y., Yamagata, M., Chu, M.W., Meister, M., Sanes, J.R., 2011. Retinal ganglion cells with distinct directional preferences differ in molecular identity, structure, and central projections. J. Neurosci. 31, 7753-7762.

Kenny, A.J., Stephenson, S.L., 1988. Role of endopeptidase-24.11 in the inactivation of atrial natriuretic peptide. FEBS Lett. 232, 1-8.

Kenny, P.A., Bissell, M.J., 2007. Targeting TACE-dependent EGFR ligand shedding in breast cancer. J. Clin. Invest. 117, 337-345.

Kerkela, E., Ala-Aho, R., Jeskanen, L., Rechardt, O., Grenman, R., Shapiro, S.D., Kahäri, V.M., Saarialho-Kere, U., 2000. Expression of human macrophage metalloelastase (MMP-12) by tumor cells in skin cancer. J. Invest. Dermatol. 114, 1113-1119.

Kerkela, E., Bohling, T., Herva, R., Uria, J.A., Saarialho-Kere, U., 2001. Human macrophage metalloelastase (MMP-12) expression is induced in chondrocytes during fetal development and malignant transformation. Bone 29, 487-493.

Kessenbrock, K., Plaks, V., Werb, Z., 2010. Matrix metalloproteinases: regulators of the tumor microenvironment. Cell 141, 52-67.

Kessler, E., Takahara, K., Biniaminov, L., Brusel, M., Greenspan, D.S., 1996. Bone morphogenetic protein-1: the type I procollagen C-proteinase. Science 271, 360-362.

Kest, B., Orlowski, M., Bodnar, R.J., 1991. Increases in opioid-mediated swim antinociception following endopeptidase 24.15 inhibition. Physiol. Behav. 50 , $843-845$

Kevorkian, L., Young, D.A., Darrah, C., Donell, S.T., Shepstone, L., Porter, S., Brockbank, S.M., Edwards, D.R., Parker, A.E., Clark, I.M., 2004. Expression profiling of metalloproteinases and their inhibitors in cartilage. Arthritis Rheum. 50, 131-141.

Khokha, R., Zimmer, M.J., Wilson, S.M., Chambers, A.F., 1992. Up-regulation of TIMP-1 expression in B16-F10 melanoma cells suppresses their metastatic ability in chick embryo. Clin. Exp. Metastasis 10, 365-370.

Kiili, M., Cox, S.W., Chen, H.Y., Wahlgren, J., Maisi, P., Eley, B.M., Salo, T., Sorsa, T., 2002. Collagenase-2 (MMP-8) and collagenase-3 (MMP-13) in adult periodontitis: molecular forms and levels in gingival crevicular fluid and immunolocalisation in gingival tissue. J. Clin. Periodontol. 29 , 224-232.

Kim, H.E., Dalal, S.S., Young, E., Legato, M.J., Weisfeldt, M.L., D’Armiento, J., 2000. Disruption of the myocardial extracellular matrix leads to cardiac dysfunction. J. Clin. Invest. 106, 857-866.

Kim, K.C., Lee, C.H., 2005. MAP kinase activation is required for the MMP-9 induction by TNF-stimulation. Arch. Pharm. Res. $28,1257-1262$.

Kim, E.S., Sohn, Y.W., Moon, A., 2007. TGF-beta-induced transcriptional activation of MMP-2 is mediated by activating transcription factor (ATF)2 in human breast epithelial cells. Cancer Lett. 252, 147-156.

Kim, S.T., Kim, E.M., Choi, J.H., Son, H.J., Ji, I.J., Joh, T.H., Chung, S.J., Hwang, O., 2010. Matrix metalloproteinase-3 contributes to vulnerability of the nigral dopaminergic neurons. Neurochem. Int. 56, 161-167.

Kim, E.J., Cho, H.J., Park, D., Kim, J.Y., Kim, Y.B., Park, T.G., Shim, C.K., Oh, Y.K., 2011a. Antifibrotic effect of MMP-13-encoding plasmid DNA delivered using polyethylenimine shielded with hyaluronic acid. Mol. Ther. 19, 355-361.

Kim, K.S., Choi, H.M., Lee, Y.A., Choi, I.A., Lee, S.H., Hong, S.J., Yang, H.I., Yoo, M.C., 2011b. Expression levels and association of gelatinases MMP-2 and MMP-9 and collagenases MMP-1 and MMP-13 with VEGF in synovial fluid of patients with arthritis. Rheumatol. Int. 31, 543-547.

Kirkegaard, I., Uldbjerg, N., Oxvig, C., 2010. Biology of pregnancy-associated plasma protein-A in relation to prenatal diagnostics: an overview. Acta Obstet. Gynecol. Scand. 9, 1118-1125.

Kiryu-Seo, S., Kiyama, H., 2004. DINE (damage induced neuronal endopeptidase). Protein Pept. Lett. 11, 451-460.

Kiryu-Seo, S., Sasaki, M., Yokohama, H., Nakagomi, S., Hirayama, T., Aoki, S., Wada, K., Kiyama, H., 2000. Damage-induced neuronal endopeptidase (DINE) is a unique metallopeptidase expressed in response to neuronal damage and activates superoxide scavengers. Proc. Natl. Acad. Sci. USA 97, 4345-4350.

Kitagawa, Y., Kunimi, K., Uchibayashi, T., Sato, H., Namiki, M., 1999. Expression of messenger RNAs for membrane-type 1, 2, and 3 matrix metalloproteinases in human renal cell carcinomas. J. Urol. 162, 905-909.

Klatt, A.R., Paul-Klausch, B., Klinger, G., Kühn, G., Renno, J.H., Banerjee, M., Malchau, G., Wielckens, K., 2009. A critical role for collagen II in cartilage matrix degradation: collagen II induces pro-inflammatory cytokines and MMPs in primary human chondrocytes. Orthop. Res. 27, 65-70.

Klawitter, M., Quero, L., Bertolo, A., Mehr, M., Stoyanov, J., Nerlich, A.G., Klasen, J., Aebli, N., Boos, N., Wuertz, K., 2011. Human MMP28 expression is unresponsive to inflammatory stimuli and does not correlate to the grade of intervertebral disc degeneration. J. Negat. Results Biomed. 10, 9.

Klein, T., Bischoff, R., 2011. Active metalloproteases of the A Disintegrin and Metalloprotease (ADAM) family: biological function and structure. J. Proteome Res. 10, 17-33.

Knäuper, V., Wilhelm, S.M., Seperack, P.K., DeClerk, Y.A., Langley, K.E., Osthues, A., Tschesche, H., 1993. Direct activation of human neutrophil procollagenase by recombinant stromelysin. Biochem. J. 295, 581-586.

Knäuper, V., Lopez-Otin, C., Smith, B., Knight, G., Murphy, G., 1996. Biochemical characterization of human collagenase-3. J. Biol. Chem. 271, 1544-1550. 
Knäuper, V., Cowell, S., Smith, B., Lopez-Otin, C., O'Shea, M., Morris, H., Zardi, L., Murphy, G., 1997. The role of the C-terminal domain of human collagenase-3 (MMP-13) in the activation of procollagenase-3, substrate specificity, and tissue inhibitor of metalloproteinase interaction. J. Biol. Chem. 272, 76087616.

Knox, J.B., Sukhova, G.K., Whittemore, A.D., Libby, P., 1997. Evidence for altered balance between matrix metalloproteinases and their inhibitors in human aortic diseases. Circulation 95, 205-212.

Kobayashi, T., Chung, U.I., Shipani, E., Starbuck, M., Karsenty, G., Katagiri, T., Goad, D.L., Lanske, B., Kronenberg, H.M., 2002. PTHrP and Indian hedgehog control differentiation of growth plate chondrocytes at multiple steps. Development 129, 2977-2986.

Kobayashi, K., Nishioka, M., Kohno, T., Nakamoto, M., Maeshima, A., Aoyagi, K., Sasaki, H., Takenoshita, S., Sugimura, H., Yokota, J., 2004. Identification of genes whose expression is upregulated in lung adenocarcinoma cells in comparison with type II alveolar cells and bronchiolar epithelial cells in vivo. Oncogene 23, 3089-3096.

Kodali, R., Hajjou, M., Berman, A.B., Bansal, M.B., Zhang, S., Pan, J.J., Schecter, A.D., 2006. Chemokines induce matrix metalloproteinase-2 through activation of epidermal growth factor receptor in arterial smooth muscle cells. Cardiovasc. Res. 69, 706-715.

Koehne, P., Schäper, C., Graf, K., Kunkel, G., 1998. Neutral endopeptidase 24.11: its physiologic and possibly pathophysiologic role in inflammation with special effect on respiratory inflammation. Allergy 53, 1023-1042.

Köhrmann, A., Kammerer, U., Kapp, M., Dietl, J., Anacker, J., 2009. Expression of matrix metalloproteinases (MMPs) in primary human breast cancer and breast cancer cell lines: new findings and review of the literature. BMC Cancer 9, 188.

Kojro, E., Fahrenholz, F., 2005. The non-amyloidogenic pathway: structure and function of $\alpha$-secretases. Subcell. Biochem. 38, 105-127.

Kolkenbrock, H., Essers, L., Ulbrich, N., Will, H., 1999. Biochemical characterization of the catalytic domain of membrane-type 4 matrix metalloproteinase. Biol. Chem. 380, 1103-1108.

Komori, K., Nonaka, T., Okada, A., Kinoh, H., Hayashita-Kinoh, H., Yoshida, N., Yana, I., Seiki, M., 2004. Absence of mechanical allodynia and Abeta-fiber sprouting after sciatic nerve injury in mice lacking membrane-type 5 matrix metalloproteinase. FEBS Lett. 557, $125-128$.

Konstantinopoulos, P.A., Karamouzis, M.V., Papatsoris, A.G., Papavassiliou, A.G., 2008. Matrix metalloproteinase inhibitors as anticancer agents. Int. J. Biochem. Cell Biol. 40, 1156-1168.

Konttinen, Y.T., Ainola, M., Valleala, H., Ma, J., Ida, H., Mandelin, J., Kinne, R.W., Santavirta, S., Sorsa, T., Lopez-Otin, C., Takagi, M., 1999a. Analysis of 16 different matrix metalloproteinases (MMP-1 to MMP-20) in the synovial membrane: different profiles in trauma and rheumatoid arthritis. Ann. Rheum. Dis. 58, 691-697.

Konttinen, Y.T., Salo, T., Hanemaaijer, R., Valleala, H., Sorsa, T., Sutinen, M., Ceponis, A., Xu, J.W., Santavirta, S., Teronen, O., Lopez-Otin, C., 1999b. Collagenase-3 (MMP-13) and its activators in rheumatoid arthritis: localization in the pannus-hard tissue junction and inhibition by alendronate. Matrix Biol. 18, 401-412.

Koo, B.H., Han, J.H., Yeom, Y.I., Kim, D.S., 2010. Thrombin-dependent MMP-2 activity is regulated by heparan sulfate. J. Biol. Chem. 285, 41270-41279.

Koong, A.C., Denko, N.C., Hudson, K.M., Schindler, C., Swiersz, L., Koch, C., Evans, S., Ibrahim, H., Le, Q.T., Terris, D.J., Giaccia, A.J., 2000. Candidate genes for the hypoxic tumor phenotype. Cancer Res. 60, 883-887.

Koppen, M., Metodier, M., Casari, G., 2007. Variable and tissue-specific subunit composition of mitochondrial m-AAA protease complexes linked to hereditary spastic paraplegia. Mol. Cell. Biol. 27, 758-767.

Korpi, J.T., Kervinen, V., Mäklin, H., Väänänen, A., Lahtinen, M., Läärä, E., Ristimaki, A., Thomas, G., Ylipalosaari, M., Åström, P., Lopez-Otin, C., Sorsa, T., Kantela, S., Pirilä, E., Salo, T., 2008. Collagenase-2 (matrix metalloproteinase-8) plays a protective role in tongue cancer. Br. J. Cancer $98,766-775$.

Koshikawa, N., Giannelli, G., Cirulli, V., Miyazaki, K., Quaranta, V., 2000. Role of cell surface metalloprotease MT1-MMP in epithelial cell migration over laminin-5. J. Cell Biol. 148, 615-624.

Koslowski, R., Knoch, K.P., Wenzel, K.W., 1998. Proteinases and proteinase inhibitors during the development of pulmonary fibrosis in rat. Clin. Chim. Acta $271,45-56$

Kotra, L.P., Cross, J.B., Shimura, Y., Fridman, R., Schlegel, H.B., Mobashery, S., 2001. Insight into the complex and dynamic process of activation of matrix metalloproteinases. J. Am. Chem. Soc. 123, 3108-3113.

Krampert, M., Bloch, W., Sasaki, T., Bugnon, P., Rülicke, T., Wolf, E., Aumailley, M., Parks, W.C., Werner, S., 2004. Activities of the matrix metalloproteinase stromelysin-2 (MMP-10) in matrix degradation and keratinocyte organization in wounded skin. Mol. Biol. Cell 15, 5242-5254.

Krueger, S., Hundertmark, T., Kalinski, T., Peitz, U., Wex, T., Malfertheiner, P., Naumann, M., Roessner, A., 2006. Helicobacter pylori encoding the pathogenicity island activates matrix metalloproteinase 1 in gastric epithelial cells via JNK and ERK. J. Biol. Chem. 281, $2868-2875$.

Kühn, K., 1995. Basement membrane (type IV) collagen. Matrix Biol. 14, 439-445.

Kumar, B., Koul, S., Petersen, J., Khandrika, L., Hwa, J.S., Meacham, R.B., Wilson, S., Koul, H.K., 2010. p38 mitogen-activated protein kinase-driven MAPKAPK2 regulates invasion of bladder cancer by modulation of MMP-2 and MMP-9 activity. Cancer Res. 70, 832-841.

Kuno, K., Okada, Y., Kawashima, H., Nakamura, H., Miyasaka, M., Ohno, H., Matsushima, K., 2000. ADAMTS-1 cleaves a cartilage proteoglycan, aggrecan. FEBS Lett. 478, 241-245.

Kuropkat, C., Duenne, A.A., Herz, U., Renz, H., Werner, J.A., 2004. Significant correlation of matrix metalloproteinase and macrophage colony-stimulating factor serum concentrations in patients with head and neck cancer. Neoplasma 51, 375-378.

Kurzepa, J., Bartosik-Psujek, H., Suchozebrska-Jesionek, D., Rejdak, K., Stryjecka-Zimmer, M., Stelmasiak, Z., 2005. Role of matrix metalloproteinases in the pathogenesis of multiple sclerosis. Neurol. Neurochir. Pol. 39, 63-67.

Kushlinsky, N.E., Solovyov, Y.N., Babkina, I.V., Gershtein, E.S., Bulicheva, I.V., 2010. Matrix metalloproteinases 2, 7, 9 and tissue inhibitor of matrix metalloproteinase-1 in the sera of patients with bone tumors. Bull. Exp. Biol. Med. 149, 233-235.

Kuula, H., Salo, T., Pirilä, E., Hagström, J., Luomanen, M., Gutierrez-Fernandez, A., Romanos, G.E., Sorsa, T., 2008. Human beta-defensin-1 and -2 and matrix metalloproteinase-25 and -26 expression in chronic and aggressive periodontitis and in peri-implantitis. Arch. Oral Biol. 53, 175-186.

Kwon, Y., Cukierman, E., Godwin, AK., 2011. Differential expressions of adhesive molecules and proteases define mechanisms of ovarian tumor cell matrix penetration/invasion. PLoS One 6, e18872.

Lafleur, M.A., Handsley, M.M., Knäuper, V., Murphy, G., Edwards, D.R., 2002. Endothelial tubulogenesis within fibrin gels specifically requires the activity of membrane-type-matrix metalloproteinases (MT-MMPs). J. Cell Sci. 115, 3427-3438.

Lagarrigue, F., Dupuis-Coronas, S., Ramel, D., Delsol, G., Tronchère, H., Payrastre, B., Gaits-Iacovoni, F., 2010. Matrix metalloproteinase-9 is upregulated in nucleophosmin-anaplastic lymphoma kinase-positive anaplastic lymphomas and activated at the cell surface by the chaperone heat shock protein 90 to promote cell invasion. Cancer Res. 70, 6978-6987.

Lagente, V., Le Quement, C., Boichot, E., 2009. Macrophage metalloelastase (MMP-12) as a target for inflammatory respiratory diseases. Expert Opin. Ther. Targets 13, 287-295.

Laigaard, J., Sorensen, T., Frohlich, C., Pedersen, B.N., Christiansen, M., Schiott, K., Uldbjerg, N., Albrechtsen, R., Clausen, H.V., Ottesen, B., Wewer, U.M., 2003. ADAM12: a novel first-trimester maternal serum marker for Down syndrome. Prenat. Diagn. 23, 1086-1091.

Lang, R., Braun, M., Sounni, N.E., Noel, A., Frankenne, F., Foidart, J.M., Bode, W., Maskos, K., 2004. Crystal structure of the catalytic domain of MMP-16/MT3MMP: characterization of MT-MMP specific features. J. Mol. Biol. 336, 213-225.

Langers, A.M., Verspaget, H.W., Hommes, D.W., Sier, C.F., 2011. Single-nucleotide polymorphisms of matrix metalloproteinases and their inhibitors in gastrointestinal cancer. World J. Gastrointest. Oncol. 3, 79-98.

Lanone, S., Zheng, T., Zhu, Z., Liu, W., Lee, C.G., Ma, B., Chen, Q., Homer, R.J., Wang, J., Rabach, L.A., Rabach, M.E., Shipley, J.M., Shapiro, S.D., Senior, R.M., Elias, J.A., 2002. Overlapping and enzyme-specific contributions of matrix metalloproteinases-9 and -12 in IL-13-induced inflammation and remodeling. J. Clin. Invest. 110, 463-474.

LaPan, P., Brady, J., Grierson, C., Fleming, M., Miller, D., Sypek, J., Fu, B., 2010. Optimization of total protein and activity assays for the detection of MMP-12 in induced human sputum. BMC Pulm. Med. 10, 40 
Lappe-Siefke, C., Goebbels, S., Gravel, M., Nicksch, E., Lee, J., Braun, P.E., Griffiths, I.R., Nave, K.A., 2003. Disruption of Cnp1 uncouples oligodendroglial functions in axonal support and myelination. Nat. Genet. 33, 366-374.

Larsen, P.H., Yong, V.W., 2004. The expression of matrix metalloproteinase-12 by oligodendrocytes regulates their maturation and morphological differentiation. J. Neurosci. 24, 7597-7603.

Larsen, P.H., DaSilva, A.G., Conant, K., Yong, V.W., 2006. Myelin formation during development of the CNS is delayed in matrix metalloproteinase-9 and-12 null mice. J. Neurosci. 26, 2207-2214.

La Russa, A., Cittadella, R., De Marco, E.V., Valentino, P., Andreoli, V., Trecroci, F., Latorre, V., Gambardella, A., Quattrone, A., 2010. Single nucleotide polymorphism in the MMP-9 gene is associated with susceptibility to develop multiple sclerosis in an Italian case-control study. J. Neuroimmunol. 225, 175-179.

Lauricella-Lefebvre, M.A., Castronovo, V., Sato, H., Seiki, M., French, D.L., Merville, M.P., 1993. Stimulation of the 92-kDa type IV collagenase promoter and enzyme expression in human melanoma cells. Invasion Metastasis 13, 289-300.

Laursen, L.S., Overgaard, M.T., Søe, R., Boldt, H.B., Sottrup-Jensen, L., Giudice, L.C., Conover, C.A., Lee, S., Russo, D., Redman, C.M., 2000. The Kell blood group system: Kell and XK membrane proteins. Semin. Hematol. 37, 113-121.

Lavigne, M.C., Thakker, P., Gunn, J., Wong, A., Miyashiro, J.S., Wasserman, A.M., 2004. Human bronchial epithelial cells express and secrete MMP-12. Biochem. Biophys. Res. Commun. 324, 534-546.

Laxton, R.C., Hu, Y., Duchene, J., Zhang, F., Zhang, Z., Leung, K.Y., Xiao, Q., Scotland, R.S., Hodgkinson, C.P., Smith, K., Willet, J., Lopez-Otin, C., Simpson, I.A., Kiechl, S., Ahluwalia, A., Xu, Q., Ye, S., 2009. A role of matrix metalloproteinase-8 in atherosclerosis. Circ. Res. 105, 921-929.

Leclerc, O., Lagente, V., Planquois, J.M., Berthelier, C., Artola, M., Eichholtz, T., Bertrand, C.P., Schmidlin, F., 2006. Involvement of MMP-12 and phosphodiesterase type 4 in cigarette smoke-induced inflammation in mice. Eur. Respir. J. 27, 1102-1109.

Lee, S., Russo, D., Redman, C.M., 2000. The Kell blood group system: Kell and XK membrane proteins. Semin. Hematol. 37, 113-121.

Lee, T., Esemuede, N., Sumpio, B.E., Gahtan, V., 2003. Thrombospondin-1 induces matrix metalloproteinase-2 activation in vascular smooth muscle cells. J. Vasc. Surg. 38, 147-154

Lee, S., Jilani, S.M., Nikolova, G.V., Carpizo, D., Iruela-Arispe, M.L., 2005. Processing of VEGF-A by matrix metalloproteinases regulates bioavailability and vascular patterning in tumors. J. Cell Biol. 169, 681-691.

Lee, H., Tsygankov, A.Y., 2010. c-Cbl regulates glioma invasion through matrix metalloproteinase 2. J. Cell. Biochem. 111, $1169-1178$.

Lefebvre, O., Regnier, C., Chenard, M.P., Wendling, C., Chambon, P., Basset, P., Rio, M.C., 1995. Developmental expression of mouse stromelysin-3 mRNA. Development 121, 947-955.

Leib, S.L., Leppert, D., Clements, J., Tauber, M.G., 2000. Matrix metalloproteinases contribute to brain damage in experimental pneumococcal meningitis. Infect. Immun. 68, 615-620.

Lemaitre, V., O’Byrne, T.K., Borczuk, A.C., Okada, Y., Tall, A.R., D’Armiento, J., 2001. ApoE knockout mice expressing human matrix metalloproteinase-1 in macrophages have less advanced atherosclerosis. J. Clin. Invest. 107, 1227-1234.

Lemaitre, V., Kim, H.E., Forney-Prescott, M., Okada, Y., D’Armiento, J., 2009. Transgenic expression of matrix metalloproteinase-9 modulates collagen deposition in a mouse model of atherosclerosis. Atherosclerosis 205, 107-112.

Lemieux, G.A., Blumenkron, F., Yeung, N., Zhou, P., Williams, J., Grammer, A.C., Petrovich, R., Lipsky, P.E., Moss, M.L., Werb, Z., 2007. The low affinity IgE receptor (CD23) is cleaved by the metalloproteinase ADAM10. J. Biol. Chem. 282, 14836-14844.

Lendeckel, U., Kohl, J., Arndt, M., Carl-McGrath, S., Donat, H., Röcken, C., 2005. Increased expression of ADAM family members in human breast cancer and breast cancer cell lines. J. Cancer Res. Clin. Oncol. 131, 41-48.

Leppert, D., Leib, S.L., Grygar, C., Miller, K.M., Schaad, U.B., Hollander, G.A., 2000. Matrix metalloproteinase (MMP)-8 and MMP-9 in cerebrospinal fluid during bacterial meningitis: association with blood-brain barrier damage and neurological sequelae. Clin. Infect. Dis. $31,80-84$.

Le Quement, C., Guenon, I., Gillon, J.Y., Lagente, V., Boichot, E., 2008. MMP-12 induces IL-8/CXCL8 secretion through EGFR and ERK1/2 activation in epithelial cells. Am. J. Physiol. Lung Cell. Mol. Physiol. 294, L1076-1084.

Lesueur, F., Oudot, T., Heath, S., Foglio, M., Lathrop, M., Prud'homme, J.F., Fischer, J., 2007. ADAM33, a new candidate for psoriasis susceptibility. PLoS One 2 , e906.

Lettau, I., Hattermann, K., Held-Feindt, J., Brauer, R., Sedlacek, R., Mentlein, R., 2010. Matrix metalloproteinase-19 is highly expressed in astroglial tumors and promotes invasion of glioma cells. J. Neuropathol. Exp. Neurol. 69, 215-223.

Levi, E., Fridman, R., Miao, H.Q., Ma, Y.S., Yayon, A., Vlodavsky, I., 1996. Matrix metalloproteinase 2 releases active soluble ectodomain of fibroblast growth factor receptor 1. Proc. Natl. Acad. Sci. USA 93, 7069-7074.

Levy, G.G., Nichols, W.C., Lian, E.C., Foroud, T., McClintick, J.N., McGee, B.M., Yang, A.Y., Siemieniak, D.R., Stark, K.R., Gruppo, R., Sarode, R., Shurin, S.B., Chandrasekaran, V., Stabler, S.P., Sabio, H., Bouhassira, E.E., Upshaw Jr., J.D., Ginsburg, D., Tsai, H.M., 2001. Mutations in a member of the ADAMTS gene family cause thrombotic thrombocytopenic purpura. Nature 413, 488-494.

Li, S.W., Sieron, A.L., Fertala, A., Hojima, Y., Arnold, W.V., Prockop, D.J., 1996. The C-proteinase that processes procollagens to fibrillar collagens is identical to the protein previously identified as bonemorphogenic protein-1. Proc. Natl. Acad. Sci. USA 93, 5127-5130.

Li, Q., Park, P.W., Wilson, C.L., Parks, W.C., 2002. Matrilysin shedding of syndecan-1 regulates chemokine mobilization and transepithelial efflux of neutrophils in acute lung injury. Cell 111, 635-646.

Li, X., Xu, L., Gao, H., Li, B., Cheng, M., 2008. Effects of grape seed proanthocyanidins extracts on AGEs and expression of bone morphogenetic protein-7 in diabetic rats. J. Nephrol. 21, 722-733.

Li, A.E., Liu, C.J., 2010. The role of ADAMTSs in arthritis. Protein \& Cell 1, 33-47.

Li, Z., Xu, X., Bai, L., Chen, W., Lin, Y., 2011. Epidermal growth factor receptor-mediated tissue transglutaminase overexpression couples acquired tumor necrosis factor-related apoptosis-inducing ligand resistance and migration through c-FLIP and MMP-9 proteins in lung cancer cells. J. Biol. Chem. 286 21164-21172.

Lian, X., Qin, Y., Hossain, S.A., Yang, L., White, A., Xu, H., Shipley, J.M., Li, T., Senior, R.M., Du, H., Yan, C., 2005. Overexpression of Stat3C in pulmonary epithelium protects against hyperoxic lung injury. J. Immunol. 174, 7250-7256.

Liang, J., Liu, E., Yu, Y., Kitajima, S., Koike, T., Jin, Y., Morimoto, M., Hatakeyama, K., Asada, Y., Watanabe, T., Sasaguri, Y., Watanabe, S., Fan, J., 2006. Macrophage metalloelastase accelerates the progression of atherosclerosis in transgenic rabbits. Circulation 113, 1993-2001.

Libby, P., 2002. Inflammation in atherosclerosis. Nature 420, 868-874.

Libby, P., Aikawa, M., Jain, M.K., 2006. Vascular endothelium and atherosclerosis. Handb. Exp. Pharmacol., $285-306$.

Lida, J., Pei, D., Kang, T., Simpson, M.A., Herlyn, M., Furcht, L.T., McCarthy, J.B., 2001. Melanoma chondroitin sulfate proteoglycan regulates matrix metalloproteinase-dependent human melanoma invasion into type I collagen. J. Biol. Chem. 276, $18786-18794$.

Li de, Q., Shang, T.Y., Kim, H.S., Solomon, A., Lokeshwar, B.L., Pflugfelder, S.C., 2003. Regulated expression of collagenases MMP-1, -8, and -13 and stromelysins MMP-3, -10 , and -11 by human corneal epithelial cells. Invest. Ophthalmol. Vis. Sci. 44, 2928-2936.

Lijnen, H.R., Ugwu, F., Bini, A., Collen, D., 1998. Generation of an angiostatin-like fragment from plasminogen by stromelysin-1 (MMP-3). Biochemistry 37, 4699-4702.

Lin, T.M., Halbert, S.P., Spellacy, W.N., 1974. Measurement of pregnancy-associated plasma proteins during human gestation. J. Clin. Invest. 54, 576-582.

Lin, H.C., Chang, J.H., Jain, S., Gabison, E.E., Kure, T., Kato, T., Fukai, N., Azar, D.T., 2001. Matrilysin cleavage of corneal collagen type XVIII NC1 domain and generation of a 28-kDa fragment. Invest. Ophthalmol. Vis. Sci. 42, 2517-2524.

Lin, M.H., Liu, S.Y., Su, H.J., Liu, Y.C., 2006. Functional role of matrix metalloproteinase-28 in the oral squamous cell carcinoma. Oral Oncol. 42, 907-913.

Lin, C.C., Kuo, C.T., Cheng, C.Y., Wu, C.Y., Lee, C.W., Hsieh, H.L., Lee, I.T., Yang, C.M., 2009. IL-1 beta promotes A549 cell migration via MAPKs/AP-1- and NFkappaB-dependent matrix metalloproteinase-9 expression. Cell. Signal. 21, 1652-1662. 
Lin, L., Bankaitis, E., Heimbach, L., Li, N., Abrink, M., Pejler, G., An, L., Diaz, L.A., Werb, Z., Liu, Z., 2011a. Dual targets for mouse mast cell protease-4 in mediating tissue damage in experimental bullous pemphigoid. J. Biol. Chem. 286, 37358-37367.

Lin, F., Wang, X., Jie, Z., Hong, X., Li, X., Wang, M., Yu, Y., 2011b. Inhibitory effects of miR-146b-5p on cell migration and invasion of pancreatic cancer by targeting MMP-16. J. Huazhong Univ. Sci. Technolog. Med. Sci. 31, 509-514.

Linask, K.K., Han, M., Cai, D.H., Brauer, P.R., Maisastry, S.M., 2005. Cardiac morphogenesis: matrix metalloproteinase coordination of cellular mechanisms underlying heart tube formation and directionality of looping. Dev. Dyn. 233, 739-753.

Lindsey, M.L., Escobar, G.P., Dobrucki, L.W., Goshorn, D.K., Bouges, S., Mingoia, J.T., McClister Jr., D.M., Su, H., Gannon, J., MacGillivray, C., Lee, R.T., Sinusas, A.J., Spinale, F.G., 2006. Matrix metalloproteinase-9 gene deletion facilitates angiogenesis after myocardial infarction. Am. J. Physiol. Heart Circ. Physiol. 290, H232-H239.

Lindy, O., Konttinen, Y.T., Sorsa, T., Ding, Y., Santavirta, S., Ceponis, A., Lopez-Otin, C., 1997. Matrix metalloproteinase 13 (collagenase 3) in human rheumatoid synovium. Arthritis Rheum. 40, 1391-1399.

Liotta, L.A., Tryggvason, K., Garbisa, S., Hart, I., Foltz, C.M., Shafie, S., 1980. Metastatic potential correlates with enzymatic degradation of basement membrane collagen. Nature 284, 67-68.

Lipman, M.L., Panda, D., Bennett, H.P., Henderson, J.E., Shane, E., Shen, Y., Goltzman, D., Karaplis, A.C., 1998. Cloning of human PEX cDNA. Expression, subcellular localization, and endopeptidase activity. J. Biol. Chem. 273, 13729-13737.

Littlepage, L.E., Sternlicht, M.D., Rougier, N., Phillips, J., Gallo, E., Yu, Y., Williams, K., Brenot, A., Gordon, J.I., Werb, Z., 2010. Matrix metalloproteinases contribute distinct roles in neuroendocrine prostate carcinogenesis, metastasis, and angiogenesis progression. Cancer Res. 70, $2224-2234$.

Liu, Z., Shipley, J.M., Vu, T.H., Zhou, X., Diaz, L.A., Werb, Z., Senior, R.M., 1998. Gelatinase B-deficient mice are resistant to experimental bullous pemphigoid. J. Exp. Med. 188, 475-482.

Liu, M., Sun, H., Wang, X., Koike, T., Mishima, H., Ikeda, K., Watanabe, T., Ochiai, N., Fan, J., 2004. Increased expression of macrophage elastase (MMP-12) is associated with rheumatoid arthritis. Arthritis Rheum. 50, 3112-3117.

Liu, Z., Li, N., Diaz, L.A., Shipley, M., Senior, R.M., Werb, Z., 2005. Synergy between a plasminogen cascade and MMP-9 in autoimmune disease. J. Clin. Invest. $115,879-887$

Liu, B., Zhou, Z., 2008. Lamin A/C, laminopathies and premature ageing. Histol. Histopathol. 23, 747-763.

Liu, W., Hendren, J., Qin, X.J., Shen, J., Liu, K.J., 2009. Normobaric hyperoxia attenuates early blood-brain barrier disruption by inhibiting MMP-9-mediated occludin degradation in focal cerebral ischemia. J. Neurochem. 108, 811-820.

Liu, L., Wu, J., Ying, Z., Chen, B., Han, A., Liang, Y., Song, L., Yuan, J., Li, J., Li, M., 2010. Astrocyte elevated gene-1 upregulates matrix metalloproteinase-9 and induces human glioma invasion. Cancer Res. 70, 3750-3759.

Liu, L., Wu, J., Wu, C., Wang, Y., Zhong, R., Zhang, X., Tan, W., Nie, S., Miao, X., Lin, D., 2011. A functional polymorphism (-1607 1G $\rightarrow 2$ G) in the matrix metalloproteinase-1 promoter is associated with development and progression of lung cancer. Cancer 117 (22), $5172-5181$.

Llano, E., Pendas, A.M., Freije, J.P., Nakano, A., Knäuper, V., Murphy, G., Lopez-Otin, C., 1999. Identification and characterization of human MT5-MMP, a new membrane-bound activator of progelatinase A overexpressed in brain tumors. Cancer Res. 59, 2570-2576.

Lochter, A., Galosy, S., Muschler, J., Freedman, N., Werb, Z., Bissell, M.J., 1997. Matrix metalloproteinase stromelysin-1 triggers a cascade of molecular alterations that leads to stable epithelial-to-mesenchymal conversion and a premalignant phenotype in mammary epithelial cells. J. Cell Biol. 139, 1861-1872.

Lohi, J., Wilson, C.L., Roby, J.D., Parks, W.C., 2001. Epilysin, a novel human matrix metalloproteinase (MMP-28) expressed in testis and keratinocytes and in response to injury. J. Biol. Chem. 276, 10134-10144.

Lomas, D.A., Mahadeva, R., 2002. Alpha1-antitrypsin polymerization and the serpinopathies: pathobiology and prospects for therapy. J. Clin. Invest. 110, 1585-1590.

Lombardi, F., Fasciglione, G.F., D’Apice, M.R., Vielle, A., D’Adamo, M., Sbraccia, P., Marini, S., Borgiani, P., Coletta, M., Novelli, G., 2008. Increased release and activity of matrix metalloproteinase-9 in patients with mandibuloacral dysplasia type A, a rare premature ageing syndrome. Clin. Genet. 74 , $374-383$.

Longo, G.M., Xiong, W., Greiner, T.C., Zhao, Y., Fiotti, N., Baxter, B.T., 2002. Matrix metalloproteinases 2 and 9 work in concert to produce aortic aneurysms. J. Clin. Invest. 110, 625-632.

Longo, G.M., Buda, S.J., Fiotta, N., Xiong, W., Griener, T., Shapiro, S., Baxter, B.T., 2005. MMP-12 has a role in abdominal aortic aneurysms in mice. Surgery $137,457-462$.

Lorenzl, S., Albers, D.S., Relkin, N., Ngyuen, T., Hilgenberg, S.L., Chirichigno, J., Cudkowicz, M.E., Beal, M.F., 2003. Increased plasma levels of matrix metalloproteinase-9 in patients with Alzheimer's disease. Neurochem. Int. 43, 191-196.

Lopez, B., Gonzalez, A., Diez, J., 2010. Circulating biomarkers of collagen metabolism in cardiac diseases. Circulation 121, 1645-1654.

Lottaz, D., Maurer, C.A., Hahn, D., Buchler, M.W., Sterchi, E.E., 1999. Nonpolarized secretion of human meprin alpha in colorectal cancer generates an increased proteolytic potential in the stroma. Cancer Res. 59, 1127-1133.

Luan, Y., Kong, L., Howell, D.R., Ilalov, K., Fajardo, M., Bai, X.H., Di Cesare, P.E., Goldring, M.B., Abramson, S.B., Liu, C.J., 2008. Inhibition of ADAMTS-7 and ADAMTS-12 degradation of cartilage oligomeric matrix protein by alpha-2-macroglobulin. Osteoarthritis Cartilage 16, 1413-1420.

Luchtefeld, M., Grote, K., Grothusen, C., Bley, S., Bandlow, N., Selle, T., Strüber, M., Haverich, A., Bavendiek, U., Drexler, H., Schieffer, B., 2005. Angiotensin II induces MMP-2 in a p47phox-dependent manner. Biochem. Biophys. Res. Commun. 328, 183-188.

Lum, L., Reid, M.S., Blobel, C.P., 1998. Intracellular maturation of the mouse metalloprotease disintegrin MDC15. J. Biol. Chem. 273, 26236-26247.

Lund, J., Qin, Q.P., Ilva, T., Pettersson, K., Voipio-Pulkki, L.M., Porela, P., Pulkki, K., 2003. Circulating pregnancy-associated plasma protein a predicts outcome in patients with acute coronary syndrome but no troponin I elevation. Circulation 108, 1924-1926.

Luo, Y.P., Zhong, M., Wang, L.P., Zhong, M., Sun, G.Q., Li, J., 2009. Inhibitory effects of RNA interference on MMP-24 expression and invasiveness of ovarian cancer SKOV(3) cells. Nan Fang Yi Ke Da Xue Xue Bao 29, 781-784.

Lusis, A.J., 2000. Atherosclerosis. Nature 407, 233-241.

Luttun, A., Lutgens, E., Manderveld, A., Maris, K., Collen, D., Carmeliet, P., Moons, L., 2004. Loss of matrix metalloproteinase-9 or matrix metalloproteinase-12 protects apolipoprotein E-deficient mice against atherosclerotic media destruction but differentially affects plaque growth. Circulation 109, 1408-1414.

Lynch, C.C., Hikosaka, A., Acuff, H.B., Martin, M.D., Kawai, N., Singh, R.K., Vargo-Gogola, T.C., Begtrup, J.L., Peterson, T.E., Fingleton, B., Shirai, T., Matrisian, L.M., Futakuchi, M., 2005. MMP-7 promotes prostate cancer-induced osteolysis via the solubilization of RANKL. Cancer Cell 7, 485-496.

Ma, X., Jiang, Y., Wu, A., Chen, X., Pi, R., Liu, M., Liu, Y., 2010. Berberine attenuates experimental autoimmune encephalomyelitis in C57 BL/6 mice. PLoS One 5 , e13489.

Ma, S., Yang, D., Li, D., Tang, B., Sun, M., Yang, Y., 2011. Cardiac extracellular matrix tenascin-C deposition during fibronectin degradation. Biochem. Biophys. Res. Commun. 409, 321-327.

Madala, S.K., Pesce, J.T., Ramalingam, T.R., Wilson, M.S., Minnicozzi, S., Cheever, A.W., Thompson, R.W., Mentink-Kane, M.M., Wynn, T.A., 2010. Matrix metalloproteinase 12-deficiency augments extracellular matrix degrading metalloproteinases and attenuates IL-13-dependent fibrosis. J. Immunol. 184, 3955-3963.

Madlener, M., Werner, S., 1997. cDNA cloning and expression of the gene encoding murine stromelysin-2 (MMP-10). Gene 202, 75-81.

Madlener, M., Parks, W.C., Werner, S., 1998. Matrix metalloproteinases (MMPs) and their physiological inhibitors (TIMPs) are differentially expressed during excisional skin wound repair. Exp. Cell Res. 242, 201-210.

Maeda, S., Dean, D.D., Gomez, R., Schwartz, Z., Boyan, B.D., 2002. The first stage of transforming growth factor-1 activation is release of the large latent complex from the extracellular matrix of growth plate chondrocytes by matrix vesicle stromelysin-1 (MMP-3). Calcif. Tissue Int. $70,54-65$.

Mäkelä, M., Salo, T., Larjava, H., 1998. MMP-9 from TNF alpha-stimulated keratinocytes binds to cell membranes and type I collagen: a cause for extended matrix degradation in inflammation? Biochem. Biophys. Res. Commun. 253, 325-335. 
Malfait, A.M., Liu, R.Q., Ijiri, K., Komiya, S., Tortorella, M.D., 2002. Inhibition of ADAM-TS4 and ADAM-TS5 prevents aggrecan degradation in osteoarthritic cartilage. J. Biol. Chem. 277, 22201-22208.

Mallat, Z., 2009. Matrix metalloproteinase-8 and the regulation of blood pressure, vascular inflammation, and atherosclerotic lesion growth. Circ. Res. 105, $827-829$.

Mallya, S.K., Mookhtiar, K.A., Gao, Y., Brew, K., Dioszegi, M., Birkedal-Hansen, H., van Wart, H.E., 1990. Characterization of 58-kilodalton human neutrophil collagenase: comparison with human fibroblast collagenase. Biochemistry 29, 10628-10634.

Maltecca, F., Aghaie, A., Schroder, D.G., Schroeder, D.G., Cassina, L., Taylor, B.A., Phillips, S.J., Malaguti, M., Previtali, S., Guénet, J.L., Quattrini, A., Cox, G.A., Casari, G., 2008. The motor protein AFG3L2 is essential for axonal development. J. Neurosci. 28, 2827-2836.

Mannino, D.M., Aguayo, S.M., Petty, T.L., Redd, S.C., 2003. Low lung function and incident lung cancer in the United States: data From the First National Health and Nutrition Examination Survey follow-up. Arch. Intern. Med. 163, 1475-1480.

Manoury, B., Nenan, S., Guenon, I., Boichot, E., Planquois, J.M., Bertrand, C.P., Lagente, V., 2006. Macrophage metalloelastase (MMP-12) deficiency does not alter bleomycin-induced pulmonary fibrosis in mice. J. Inflamm. $3,2$.

Manso, H., Krug, T., Sobral, J., Albergaria, I., Gaspar, G., Ferro, J.M., Oliveira, S.A., Vicente, A.M., 2010. Variants of the Matrix Metalloproteinase-2 but not the Matrix Metalloproteinase-9 genes significantly influence functional outcome after stroke. BMC Med. Genet. 11, 40.

Maquoi, E., Sounni, N.E., Devy, L., Olivier, F., Frankenne, F., Krell, H.W., Grams, F., Foidart, J.M., Noel, A., 2004. Anti-invasive, antitumoral, and antiangiogenic efficacy of a pyrimidine-2,4,6-trione derivative, an orally active and selective matrix metalloproteinases inhibitor. Clin. Cancer Res. 10 , 4038-4047.

Marchenko, G.N., Strongin, A.Y., 2001. MMP-28, a new human matrix metalloproteinase with an unusual cysteine-switch sequence is widely expressed in tumors. Gene 265, 87-93.

Marchenko, G.N., Ratnikov, B.I., Rozanov, D.V., Godzik, A., Deryugina, E.I., Strongin, A.Y., 2001. Characterization of matrix metalloproteinase-26, a novel metalloproteinase widely expressed in cancer cells of epithelial origin. Biochem. J. 356, 705-718.

Marchenko, G.N., Marchenko, N.D., Strongin, A.Y., 2003. The structure and regulation of the human and mouse matrix metalloproteinase-21 gene and protein. Biochem. J. 372, 503-515.

Mari, B.P., Anderson, I.C., Mari, S.E., Ning, Y., Lutz, Y., Kobzik, L., Shipp, M.A., 1998. Stromelysin-3 is induced in tumor/stroma cocultures and inactivated via a tumor-specific and basic fibroblast growth factor-dependent mechanism. J. Biol. Chem. 273, 618-626.

Marini, S., Fasciglione, G.F., De Sanctis, G., D’Alessio, S., Politi, V., Coletta, M., 2000. Cleavage of bovine collagen I by neutrophil collagenase MMP-8. Effect of $\mathrm{pH}$ on the catalytic properties as compared to synthetic substrates. J. Biol. Chem. 275, 18657-18663.

Martin, M.D., Matrisian, L.M., 2007. The other side of MMPs: protective roles in tumor progression. Cancer Metastasis Rev. $26,717-724$.

Maskos, K., 2005. Crystal structures of MMPs in complex with physiological and pharmacological inhibitors. Biochimie 87, $249-263$.

Matrisian, L.M., Bowden, G.T., Krieg, P., Furstenberger, G., Briand, J.-P., Leroy, P., Breathnach, R., 1986. The mRNA coding for the secreted protease transin is expressed more abundantly in malignant than in benign tumors. Proc. Natl. Acad. Sci. USA 83, 9413-9417.

Matsas, R., Fulcher, I.S., Kenny, A.J., Turner, A.J., 1983. Substance P and [Leu]enkephalin are hydrolyzed by an enzyme in pig caudate synaptic membranes that is identical with the endopeptidase of kidney microvilli. Proc. Natl. Acad. Sci. USA 80, 3111-3115.

Matsumoto, S., Kobayashi, T., Katoh, M., Saito, S., Ikeda, Y., Kobori, M., Masuho, Y., Watanabe, T., 1998. Expression and localization of matrix metalloproteinase-12 in the aorta of cholesterol-fed rabbits: relationship to lesion development. Am. J. Pathol. 153, 109-119.

Matsumoto, H., Niimi, A., Takemura, M., Ueda, T., Minakuchi, M., Tabuena, R., Chin, K., Mio, T., Ito, Y., Muro, S., Hirai, T., Morita, S., Fukuhara, S., Mishima, M. 2005. Relationship of airway wall thickening to an imbalance between matrix metalloproteinase-9 and its inhibitor in asthma. Thorax $60,277-281$.

Matsumoto, Y., Park, I.K., Kohyama, K., 2009. Matrix metalloproteinase (MMP)-9, but not MMP-2, is involved in the development and progression of C protein-induced myocarditis and subsequent dilated cardiomyopathy. J. Immunol. 183, 4773-4781.

Matsumura, Y., Ikegawa, R., Takaoka, M., Morimoto, S., 1990. Conversion of porcine big endothelin to endothelin by an extract from the porcine aortic endothelial cells. Biochem. Biophys. Res. Commun. 167, 203-210.

Mattout, A., Dechat, T., Adam, S.A., Goldman, R.D., Gruenbaum, Y., 2006. Nuclear lamins, diseases and aging. Curr. Opin. Cell Biol. 18, 335-341.

Matucci-Cerinic, M., Iannone, F., Carossino, A., Pignone, A., Leoncini, G., Generini, S., Lapadula, G., Cagnoni, M., 1999. Discrepant expression of neprilysin on fibroblasts in diffuse systemic sclerosis. J. Rheumatol. 26, 347-351.

Matute-Bello, G., Wurfel, M.M., Lee, J.S., Park, D.R., Frevert, C.W., Madtes, D.K., Shapiro, S.D., Martin, T.R., 2007. Essential role of MMP-12 in Fas-induced lung fibrosis. Am. J. Respir. Cell Mol. Biol. 37, 210-221.

Matziari, M., Dive, V., Yiotakis, A., 2007. Matrix metalloproteinase 11 (MMP-11; stromelysin-3) and synthetic inhibitors. Med. Res. Rev. 27, 528-552.

Mauch, S., Kolb, C., Kolb, B., Sadowski, T., Sedlacek, R., 2002. Matrix metalloproteinase-19 is expressed in myeloid cells in an adhesion-dependent manner and associates with the cell surface. J. Immunol. 168, 1244-1251.

Mazzocca, A., Coppari, R., De Franco, R., Cho, J.Y., Libermann, T.A., Pinzani, M., Toker, A., 2005. A secreted form of ADAM9 promotes carcinoma invasion through tumor-stromal interactions. Cancer Res. 65, 4728-4738.

McCawley, L.J., Matrisian, L.M., 2001. Matrix metalloproteinases: they're not just for matrix anymore. Curr. Opin. Cell Biol. 3, 534-540.

McCawley, L.J., Crawford, H.C., King Jr., L.E., Mudgett, J., Matrisian, L.M., 2004. A protective role for matrix metalloproteinase-3 in squamous cell carcinoma. Cancer Res. 64, 6965-6972.

McGuire, J.K., Li, Q., Parks, W.C., 2003. Matrilysin (matrix metalloproteinase-7) mediates E-cadherin ectodomain shedding in injured lung epithelium. Am. J. Pathol. 162, 1831-1843.

McQuibban, G.A., Gong, J.H., Tam, E.M., McCulloch, C.A., Clark-Lewis, I., Overall, C.M., 2000. Inflammation dampened by gelatinase A cleavage of monocyte chemoattractant protein-3. Science 289, 1202-1206.

Mehra, D., Sternberg, D.I., Jia, Y., Canfield, S., Lemaitre, V., Nkyimbeng, T., Wilder, J., Sonett, J., D’Armiento, J., 2010. Altered lymphocyte trafficking and diminished airway reactivity in transgenic mice expressing human MMP-9 in a mouse model of asthma. Am. J. Physiol. Lung Cell. Mol. Physiol. 298, L189-L196.

Mercer, B.A., Kolesnikova, N., Sonett, J., D’Armiento, J., 2004. Extracellular regulated kinase/mitogen activated protein kinase is upregulated in pulmonary emphysema and mediates matrix metalloproteinase-1 induction by cigarette smoke. J. Biol. Chem. 279, 17690-17696.

Meyer, E., Vollmer, J.Y., Bovey, R., Stamenkovic, I., 2005. Matrix metalloproteinases 9 and 10 inhibit protein kinase C-potentiated, p53-mediated apoptosis. Cancer Res. 65, 4261-4272.

Mewar, R., McMorris, F.A., 1997. Expression of insulin-like growth factorbinding protein messenger RNAs in developing rat oligodendrocytes and astrocytes. J. Neurosci. Res. 50, 721-728.

Michael, M., Babic, B., Khokha, R., Tsao, M., Ho, J., Pintilie, M., Leco, K., Chamberlain, D., Shepherd, F.A., 1999. Expression and prognostic significance of metalloproteinases and their tissue inhibitors in patients with small-cell lung cancer. J. Clin. Oncol. 17, 1802-1808.

Mias, C., Lairez, O., Trouche, E., Roncalli, J., Calise, D., Seguelas, M.H., Ordener, C., Piercecchi-Marti, M.D., Auge, N., Salvayre, A.N., Bourin, P., Parini, A., Cussac, D., 2009. Mesenchymal stem cells promote matrix metalloproteinase secretion by cardiac fibroblasts and reduce cardiac ventricular fibrosis after myocardial infarction. Stem Cells 27, 2734-2743.

Milner, R., Crocker, S.J., Hung, S., Wang, X., Frausto, R.F., del Zoppo, G.J., 2007. Fibronectin- and vitronectin-induced microglial activation and matrix metalloproteinase-9 expression is mediated by integrins alpha5beta1 and alphavbeta5. J. Immunol. $178,8158-8167$.

Milward, E., Kim, K.J., Szklarczyk, A., Nguyen, T., Melli, G., Nayak, M., Deshpande, D., Fitzsimmons, C., Hoke, A., Kerr, D., Griffin, J.W., Calabresi, P.A., Conant, K., 2008. Cleavage of myelin associated glycoprotein by matrix metalloproteinases. J. Neuroimmunol. 193, 140-148.

Mimori, K., Fukagawa, T., Kosaka, Y., Ishikawa, K., Iwatsuki, M., Yokobori, T., Hirasaki, S., Takatsuno, Y., Sakashita, H., Ishii, H., Sasako, M., Mori, M., 2008. A large-scale study of MT1-MMP as a marker for isolated tumor cells in peripheral blood and bone marrow in gastric cancer cases. Ann. Surg. Oncol. 15, 2934-2942. 
Mimura, T., Han, K.Y., Onguchi, T., Chang, J.H., Kim, T.I., Kojima, T., Zhou, Z., Azar, D.T., 2009. MT1-MMP-mediated cleavage of decorin in corneal angiogenesis. J. Vasc. Res. 46, 541-550.

Min, C., Eddy, S.F., Sherr, D.H., Sonenshein, G.E., 2008. NF-kappaB and epithelial to mesenchymal transition of cancer. J. Cell. Biochem. 104, 733-744.

Minond, D., Lauer-Fields, J.L., Cudic, M., Overall, C.M., Pei, D., Brew, K., Visse, R., Nagase, H., Fields, G.B., 2006. The roles of substrate thermal stability and P2 and $\mathrm{P}^{\prime}{ }^{\prime}$ subsite identity on matrix metalloproteinase triple-helical peptidase activity and collagen specificity. J. Biol. Chem. $281,38302-38313$.

Mitchell, P.G., Magna, H.A., Reeves, L.M., Lopresti-Morrow, L.L., Yocum, S.A., Rosner, P.J., Geoghegan, K.F., Hambor, J.E., 1996. Cloning, expression, and type II collagenolytic activity of matrix metalloproteinase-13 from human osteoarthritic cartilage. J. Clin. Invest. 97, 761-768.

Miura, S., Ohno, I., Suzuki, J., Suzuki, K., Okada, S., Okuyama, A., Nawata, J., Ikeda, J., Shirato, K., 2003. Inhibition of matrix metalloproteinases prevents cardiac hypertrophy induced by beta-adrenergic stimulation in rats. J. Cardiovasc. Pharmacol. 42, 174-181.

Miyamori, H., Takino, T., Seiki, M., Sato, H., 2000. Human membrane type-2 matrix metalloproteinase is defective in cell-associated activation of progelatinase A. Biochem. Biophys. Res. Commun. 267, 796-800.

Miyamoto, S., Yano, K., Sugimoto, S., Ishii, G., Hasebe, T., Endoh, Y., Kodama, K., Goya, M., Chiba, T., Ochiai, A., 2004. Matrix metalloproteinase-7 facilitates insulin-like growth factor bioavailability through its proteinase activity on insulin-like growth factor binding protein 3. Cancer Res. 64, 665-671.

Miyoshi, Y., Akagi, M., Agarwal, A.K., Namba, N., Kato-Nishimura, K., Mohri, I., Yamagata, M., Nakajima, S., Mushiake, S., Shima, M., Auchus, R.J., Taniike, M., Garg, A., Ozono, K., 2008. Severe mandibuloacral dysplasia caused by novel compound heterozygous ZMPSTE24 mutations in two Japanese siblings. Clin. Genet. 73, 535-544

Moake, J.L., 2004. von Willebrand factor, ADAMTS-13, and thrombotic thrombocytopenic purpura. Semin. Hematol. 41, 4-14.

Mochizuki, S., Shimoda, M., Shiomi, T., Fujii, Y., Okada, Y., 2004. ADAM28 is activated by MMP-7 (matrilysin-1) and cleaves insulin-like growth factor binding protein-3. Biochem. Biophys. Res. Commun. 315, 79-84.

Mochizuki, S., Okada, Y., 2007. ADAMs in cancer cell proliferation and progression. Cancer Sci. 98, 621-628.

Mohammad, M.A., Ismael, N.R., Shaarawy, S.M., El-Merzabani, M.M., 2010. Prognostic value of membrane type 1 and 2 matrix metalloproteinase expression and gelatinase A activity in bladder cancer. Int. J. Biol. Markers 25, 69-74.

Moilanen, M., Pirilä, E., Grenman, R., Sorsa, T., Salo, T., 2002. Expression and regulation of collagenase-2 (MMP-8) in head and neck squamous cell carcinomas. J. Pathol. 197, 72-81.

Moilanen, M., Sorsa, T., Stenman, M., Nyberg, P., Lindy, O., Vesterinen, J., Paju, A., Konttinen, Y.T., Stenman, U.H., Salo, T., 2003. Tumor associated trypsinogen2 (trypsinogen-2) activates procollagenases (MMP-1, -8, -13) and stromelysin-1 (MMP-3) and degrades type I collagen. Biochemistry 42, 5414-5420.

Moldovan, F., Pelletier, J.P., Hambor, J., Cloutier, J.M., Martel-Pelletier, J., 1997. Collagenase-3 (matrix metalloprotease 13) is preferentially localized in the deep layer of human arthritic cartilage in situ: in vitro mimicking effect by transforming growth factor beta. Arthritis Rheum. 40, $1653-1661$.

Molet, S., Belleguic, C., Lena, H., Germain, N., Bertrand, C.P., Shapiro, S.D., Planquois, J.M., Delaval, P., Lagente, V., 2005. Increase in macrophage elastase (MMP-12) in lungs from patients with chronic obstructive pulmonary disease. Inflamm. Res. 54, 31-36.

Molloy, K.J., Thompson, M.M., Jones, J.L., Schwalbe, E.C., Bell, P.R., Naylor, A.R., Loftus, I.M., 2004. Unstable carotid plaques exhibit raised matrix metalloproteinase-8 activity. Circulation 110, 337-343.

Monaco, S., Sparano, V., Gioia, M., Sbardella, D., Di Pierro, D., Marini, S., Coletta, M., 2006. Enzymatic processing of collagen IV by MMP-2 (gelatinase A) affects neutrophil migration and it is modulated by extracatalytic domains. Protein Sci. 15, 2805-2815.

Monea, S., Jordan, B.A., Srivastava, S., DeSouza, S., Ziff, E.B., 2006. Membrane localization of membrane type 5 matrix metalloproteinase by AMPA receptor binding protein and cleavage of cadherins. J. Neurosci. 26, 2300-2312.

Montaner, J., 2009. Blood biomarkers to guide stroke thrombolysis. Front. Biosci. 1, 200-208.

Montel, V., Kleeman, J., Agarwal, D., Spinella, D., Kawai, K., Tarin, D., 2004. Altered metastatic behavior of human breast cancer cells after experimental manipulation of matrix metalloproteinase 8 gene expression. Cancer Res. 64, 1687-1694.

Montero, I., Orbe, J., Varo, N., Beloqui, O., Monreal, J.I., Rodriguez, J.A., Diez, J., Libby, P., Paramo, J.A., 2006. C-reactive protein induces matrix metalloproteinase-1 and -10 in human endothelial cells: implications for clinical and subclinical atherosclerosis. J. Am. Coll. Cardiol. 47, 1369-1378.

Moon, S.K., Cho, S.H., Kim, K.W., Jeon, J.H., Ko, J.H., Kim, B.Y., Kim, C.H., 2007. Overexpression of membrane sialic acid-specific sialidase Neu3 inhibits matrix metalloproteinase-9 expression in vascular smooth muscle cells. Biochem. Biophys. Res. Commun. 356, 542-547.

Morancho, A., Rosell, A., Garcia-Bonilla, L., Montaner, J., 2010. Metalloproteinase and stroke infarct size: role for anti-inflammatory treatment? Ann. NY Acad. Sci. 1207, 123-133.

Morgunova, E., Tuuttila, A., Bergmann, U., Isupov, M., Lindqvist, Y., Schneider, G., Tryggvason, K., 1999. Structure of human pro-matrix metalloproteinase-2: activation mechanism revealed. Science $284,1667-1670$.

Morgunova, E., Tuuttila, A., Bergmann, U., Tryggvason, K., 2002. Structural insight into the complex formation of latent matrix metalloproteinase 2 with tissue inhibitor of metalloproteinase 2. Proc. Natl. Acad. Sci. USA 99, 7414-7419.

Moriguchi-Goto, S., Yamashita, A., Tamura, N., Soejima, K., Takahashi, M., Nakagaki, T., Goto, S., Asada, Y., 2009. ADAMTS-13 attenuates thrombus formation on type I collagen surface and disrupted plaques under flow conditions. Atherosclerosis 203, 409-416.

Morrison, C.J., Butler, G.S., Bigg, H.F., Roberts, C.R., Soloway, P.D., Overall, C.M., 2001. Cellular activation of MMP-2 (gelatinase A) by MT2-MMP occurs via a TIMP-2-independent pathway. J. Biol. Chem. 276, 47402-47410.

Morrison, C., Mancini, S., Cipollone, J., Kappelhoff, R., Roskelley, C., Overall, C.M., 2011. Microarray and proteomic analysis of breast cancer cell and osteoblast co-cultures: the role of osteoblast matrix metalloproteinase (MMP)-13 in bone metastasis. J. Biol. Chem. 276, 47402-47410.

Motrescu, E.R., Blaise, S., Etique, N., Messaddeq, N., Chenard, M.P., Stoll, I., Tomasetto, C., Rio, M.C., 2008. Matrix metalloproteinase-11/stromelysin-3 exhibits collagenolytic function against collagen VI under normal and malignant conditions. Oncogene 27, 6347-6355.

Mountain, D.J., Singh, M., Menon, B., Singh, K., 2007. Interleukin-1beta increases expression and activity of matrix metalloproteinase-2 in cardiac microvascular endothelial cells: role of PKCalpha/beta1 and MAPKs. Am. J. Physiol. Cell Physiol. 292, C867-C875.

Mu, D., Cambier, S., Fjellbirkeland, L., Baron, J.L., Munger, J.S., Kawakatsu, H., Sheppard, D., Broaddus, V.C., Nishimura, S.L., 2002. The integrin alpha(v)beta8 mediates epithelial homeostasis through MT1-MMP-dependent activation of TGF-beta1. J. Cell Biol. 157, 493-507.

Mudgett, J.S., Hutchinson, N.I., Chartrain, N.A., Forsyth, A.J., McDonnell, J., Singer, I.I., Bayne, E.K., Flanagan, J., Kawka, D., Shen, C.F., Stevens, K., Chen, H., Trumbauer, M., Visco, D.M., 1998. Susceptibility of stromelysin-1-deficient mice to collagen-induced arthritis and cartilage destruction. Arthritis Rheum. 41, 110-121.

Mueller, M.S., Harnasch, M., Kolb, C., Kusch, J., Sadowski, T., Sedlacek, R., 2000a. The murine ortholog of matrix metalloproteinase 19: its cloning, gene organization, and expression. Gene 256, 101-111.

Mueller, M.S., Mauch, S., Sedlacek, R., 2000b. Structure of the human MMP-19 gene. Gene 252, 27-37.

Mukherjee, R., Snipes, J.M., Saunders, S.M., Zavadzkas, J.A., Spinale, F.G., in press. Discordant activation of gene promoters for matrix metalloproteinases and tissue inhibitors of the metalloproteinases following myocardial infarction. J. Surg. Res.

Muller, D., Quantin, B., Gesnel, M.C., Millon-Collard, R., Abecassis, J., Breathnach, R., 1988. The collagenase gene family in humans consists of at least four members. Biochem. J. 253, 187-192.

Muller, D., Wolf, C., Abecassis, J., Millon, R., Engelmann, A., Bronner, G., Rouyer, N., Rio, M.C., Eber, M., Methlin, G., Chambon, P., Basset, P., 1993. Increased stromelysin 3 gene expression is associated with increased local invasiveness in head and neck squamous cell carcinomas. Cancer Res. 53, 165-169.

Müller, M., Beck, I.M., Gadesmann, J., Karschuk, N., Paschen, A., Proksch, E., Djonov, V., Reiss, K., Sedlacek, R., 2010. progression and increases invasion of melanoma cells. Mod. Pathol. 23, 511-521.

Munaut, C., Salonurmi, T., Kontusaari, S., Reponen, P., Morita, T., Foidart, J.M., Tryggvason, K., 1999. Murine matrix metalloproteinase 9 gene. 5'-upstream region contains cis-acting elements for expression in osteoclasts and migrating keratinocytes in transgenic mice. J. Biol. Chem. 274, 5588-5596.

Munaut, C., Boniver, J., Foidart, J.M., Deprez, M., 2002. Macrophage migration inhibitory factor (MIF) expression in human glioblastomas correlates with vascular endothelial growth factor (VEGF) expression. Neuropathol. Appl. Neurobiol. 28, 452-460. 
Munger, J.S., Huang, X., Kawakatsu, H., Griffiths, M.J., Dalton, S.L., Wu, J., Pittet, J.F., Kaminski, N., Garat, C., Matthay, M.A., Rifkin, D.B., Sheppard, D., 1999. The integrin alpha v beta 6 binds and activates latent TGF beta 1: a mechanism for regulating pulmonary inflammation and fibrosis. Cell 96, 319-328.

Muñoz, L.E., Janko, C., Schulze, C., Schorn, C., Sarter, K., Schett, G., Herrmann, M., 2010. Autoimmunity and chronic inflammation - two clearance-related steps in the etiopathogenesis of SLE. Autoimmun. Rev. 10, 38-42.

Muroski, M.E., Roycik, M.D., Newcomer, R.G., Van den Steen, P.E., Opdenakker, G., Monroe, H.R., Sahab, Z.J., Sang, Q.X., 2008. Matrix metalloproteinase-9/ gelatinase B is a putative therapeutic target of chronic obstructive pulmonary disease and multiple sclerosis. Curr. Pharm. Biotechnol. 9, 34-46.

Murphy, G., Allan, J.A., Willenbrock, F., Cockett, M.I., O'Connell, J.P., Docherty, A.J.P., 1992. The role of the C-terminal domain in collagenase and stromelysin specificity. J. Biol. Chem. 267, 9612-9618.

Murphy, G., Nguyen, Q., Cockett, M.I., Atkinson, S.J., Allan, J.A., Knight, C.G., Willenbrock, F., Docherty, A.J., 1994. Assessment of the role of the fibronectin-like domain of gelatinase A by analysis of a deletion mutant. J. Biol. Chem. 269, 6632-6636.

Murphy, G., Knäuper, V., Cowell, S., Hembry, R., Stanton, H., Butler, G., Freije, J., Pendas, A.M., Lopez-Otin, C., 1999. Evaluation of some newer matrix metalloproteinases. Ann. NY Acad. Sci. 878, 25-39.

Murphy, G., 2008. The ADAMs: signalling scissors in the tumour microenvironment. Nat. Rev. Cancer 12, 929-941.

Murray, C.J., Lopez, A.D., 1997. Alternative projections of mortality and disability by cause 1990-2020: global burden of disease study. Lancet 349, 14981504

Mysliwy, J., Dingley, A.J., Sedlacek, R., Grötzinger, J., 2006. Structural characterization and binding properties of the hemopexin-like domain of the matrixmetalloproteinase-19. Protein Expr. Purif. 46, 406-413.

Nagano, O., Murakami, D., Hartmann, D., De Strooper, B., Saftig, P., Iwatsubo, T., Nakajima, M., Shinohara, M., Saya, H., 2004. Cell-matrix interaction via CD44 is independently regulated by different metalloproteinases activated in response to extracellular Ca(2+) influx and PKC activation. J. Cell Biol. 165, 893902 .

Nagase, H., Fields, G.B., 1996. Human matrix metalloproteinase specificity studies using collagen sequence-based synthetic peptides. Biopolymers 40, 399416.

Nagase, H., Woessner Jr., J.F., 1999. Matrix metalloproteinases. J. Biol. Chem. 274, 21491-21494.

Nagase, H., 2001. Metalloproteases. Curr. Protoc. Protein Sci. 21, 2104-2124.

Nagase, H., Kashiwagi, M., 2003. Aggrecanases and cartilage matrix degradation. Arthritis Res. Ther. 5, 94-103.

Nagase, H., Visse, R., Murphy, G., 2006. Structure and function of matrix metalloproteinases and TIMPs. Cardiovasc. Res. 69, $562-573$.

Nagashima, Y., Hasegawa, S., Koshikawa, N., Taki, A., Ichikawa, Y., Kitamura, H., Misugi, K., Kihira, Y., Matuo, Y., Yasumitsu, H., Miyazaki, K., 1997. Expression of matrilysin in vascular endothelial cells adjacent to matrilysin-producing tumors. Int. J. Cancer 72, 441-445.

Nagata, K., Kiryu-Seo, S., Kiyama, H., 2006. Localization and ontogeny of damage-induced neuronal endopeptidase mRNA-expressing neurons in the rat nervous system. Neuroscience 141, 299-310.

Nakada, M., Nakamura, H., Ikeda, E., Fujimoto, N., Yamashita, J., Sato, H., Seiki, M., Okada, Y., 1999. Expression and tissue localization of membrane-type 1, 2, and 3 matrix metalloproteinases in human astrocytic tumors. Am. J. Pathol. 154, 417-428.

Nakaji, K., Ihara, M., Takahashi, C., Itohara, S., Noda, M., Takahashi, R., Tomimoto, H., 2006. Matrix metalloproteinase-2 plays a critical role in the pathogenesis of white matter lesions after chronic cerebral hypoperfusion in rodents. Stroke 37, 2816-2823.

Nakken, K.E., Nygård, S., Haaland, T., Berge, K.E., Arnkvaern, K., Ødegaard, A., Labori, K.J., Raeder, M.G., 2007. Multiple inflammatory-, tissue remodelling- and fibrosis genes are differentially transcribed in the livers of Abcb4 (-/-) mice harbouring chronic cholangitis. Scand. J. Gastroenterol. $42,1245-1255$.

Nap, A.W., Dunselman, G.A., de Goeij, A.F., Evers, J.L., Groothuis, P.G., 2004. Inhibiting MMP activity prevents the development of endometriosis in the chicken chorioallantoic membrane model. Hum. Reprod. 19, 2180-2187.

Neidhart, M., Seemayer, C.A., Hummel, K.M., Michel, B.A., Gay, R.E., Gay, S., 2003. Functional characterization of adherent synovial fluid cells in rheumatoid arthritis: destructive potential in vitro and in vivo. Arthritis Rheum. 48, 1873-1880.

Nelissen, I., Martens, E., Van den Steen, P.E., Proost, P., Ronsse, I., Opdenakker, G., 2003. Gelatinase B/matrix metalloproteinase-9 cleaves interferon-beta and is a target for immunotherapy. Brain 126, 1371-1381.

Nenan, S., Planquois, J.M., Berna, P., De Mendez, I., Hitier, S., Shapiro, S.D., Boichot, E., Lagente, V., Bertrand, C.P., 2005. Analysis of the inflammatory response induced by rhMMP-12 catalytic domain instilled in mouse airways. Int. Immunopharmacol. 5, 511-524.

Neuhold, L.A., Killar, L., Zhao, W., Sung, M.L., Warner, L., Kulik, J., Turner, J., Wu, W., Billinghurst, C., Meijers, T., Poole, A.R., Babij, P., De Gennaro, L.J., 2001. Postnatal expression in hyaline cartilage of constitutively active human collagenase-3 (MMP-13) induces osteoarthritis in mice. J. Clin. Invest. 107, 3544.

Nie, J., Pei, D., 2003. Direct activation of pro-matrix metalloproteinase-2 by leukolysin/membrane-type 6 matrix metalloproteinase/matrix metalloproteinase-25 at the Asn(109)-Tyr bond. Cancer Res. 63, 6758-6762.

Nie, R., Xie, S., Du, B., Liu, X., Deng, B., Wang, J., 2009. Extracellular matrix metalloproteinase inducer (EMMPRIN) is increased in human left ventricle after acute myocardial infarction. Arch. Med. Res. 40, 605-611.

Niimi, Y., Pawankar, R., Kawana, S., 2006. Increased expression of matrix metalloproteinase-2, matrix metalloproteinase-9 and matrix metalloproteinase-13 in lesional skin of bullous pemphigoid. Int. Arch. Allergy Immunol. 139, 104-113.

Nikkola, J., Vihinen, P., Vuoristo, M.S., Kellokumpu-Lehtinen, P., Kähäri, V.M., Pyrhönen, S., 2005. High serum levels of matrix metalloproteinase-9 and matrix metalloproteinase-1 are associated with rapid progression in patients with metastatic melanoma. Clin. Cancer Res. 11, 5158-5166.

Nilsson, L., Jonasson, L., Nijm, J., Hamsten, A., Eriksson, P., 2006. Increased plasma concentration of matrix metalloproteinase-7 in patients with coronary artery disease. Clin. Chem. 52, 1522-1527.

Nishida, Y., Miyamori, H., Thompson, E.W., Takino, T., Endo, Y., Sato, H., 2008. Activation of matrix metalloproteinase-2 (MMP-2) by membrane type 1 matrix metalloproteinase through an artificial receptor for proMMP-2 generates active MMP-2. Cancer Res. 68, 9096-9104.

Nishimura, H., Cho, C., Branciforte, D.R., Myles, D.G., Primakoff, P., 2001. Analysis of loss of adhesive function in sperm lacking cyritestin or fertilin beta. Dev. Biol. 233, 204-213.

Nishimura, H., Kim, E., Nakanishi, T., Baba, T., 2004. Possible function of the ADAM1a/ADAM2 fertilin complex in the appearance of ADAM3 on the sperm surface. J. Biol. Chem. 279, 34957-34962.

Nishizuka, I., Ichikawa, Y., Ishikawa, T., Kamiyama, M., Hasegawa, S., Momiyama, N., Miyazaki, K., Shimada, H., 2001. Matrilysin stimulates DNA synthesis of cultured vascular endothelial cells and induces angiogenesis in vivo. Cancer Lett. 173, 175-182.

Noe, V., Fingleton, B., Jacobs, K., Crawford, H.C., Vermeulen, S., Steelant, W., Bruyneel, E., Matrisian, L.M., Mareel, M., 2001. Release of an invasion promoter Ecadherin fragment by matrilysin and stromelysin-1. J. Cell Sci. 114, 111-118.

Noel, A., Santavicca, M., Stoll, I., L'Hoir, C., Staub, A., Murphy, G., Rio, M.C., Basset, P., 1995. Identification of structural determinants controlling human and mouse stromelysin-3 proteolytic activities. J. Biol. Chem. 270, 22866-22872.

Noel, A., Boulay, A., Kebers, F., Kannan, R., Hajitou, A., Calberg-Bacq, C.M., Basset, P., Rio, M.C., Foidart, J.M., 2000. Demonstration in vivo that stromelysin-3 functions through its proteolytic activity. Oncogene 19, 1605-1612.

Nolden, M., Ehses, S., Koppen, M., Bernacchia, A., Rugarli, E.I., Langer, T., 2005. The m-AAA protease defective in hereditary spastic paraplegia controls ribosome assembly in mitochondria. Cell 123, 277-289.

Norman, M.U., Lew, R.A., Smith, A.I., Hickey, M.J., 2003. Metalloendopeptidases EC 3.4.24.15/16 regulate bradykinin activity in the cerebral microvasculature. Am. J. Physiol. Heart Circ. Physiol. 284, H1942-H1948.

Noseworthy, J.H., Lucchinetti, C., Rodriguez, M., Weinshenker, B.G., 2000. Multiple sclerosis. N. Engl. J. Med. 343, 938-952.

Nuttall, R.K., Pennington, C.J., Taplin, J., Wheal, A., Yong, V.W., Forsyth, P.A., Edwards, D.R., 2003. Elevated membrane-type matrix metalloproteinases in gliomas revealed by profiling proteases and inhibitors in human cancer cells. Mol. Cancer Res. 1, 333-345. 
Nyberg, P., Heikkila, P., Sorsa, T., Luostarinen, J., Heljasvaara, R., Stenman, U.H., Pihlajaniemi, T., Salo, T., 2003. Endostatin inhibits human tongue carcinoma cell invasion and intravasation and blocks the activation of matrix metalloprotease-2, -9 , and -13. J. Biol. Chem. $278,22404-22411$.

Nygardas, P.T., Hinkkanen, A.E., 2002. Up-regulation of MMP-8 and MMP-9 activity in the BALB/c mouse spinal cord correlates with the severity of experimental autoimmune encephalomyelitis. Clin. Exp. Immunol. 128, 245-254.

Nwomeh, B.C., Liang, H.X., Cohen, I.K., Yager, D.R., 1999. MMP-8 is the predominant collagenase in healing wounds and nonhealing ulcers. J. Surg. Res. 81, $189-195$.

Oblander, S., Zhou, Z., Galvez, B.G., Starcher, B., Shannon, J.M., Durbeej, M., Arroyo, A.G., Tryggvason, K., Apte, S.S., 2005. Distinctive functions of membrane type 1 matrix-metalloprotease (MT1-MMP or MMP-14) in lung and submandibular gland development are independent of its role in pro-MMP-2 activation. Dev. Biol. 277, 255-269.

O'Connell, J., Bennett, M.W., O'Sullivan, G.C., Collins, J.K., Shanahan, F., 1999. The Fas counterattack: cancer as a site of immune privilege. Immunol. Today 20, $46-52$.

Offersen, B.V., Knap, M.M., Horsman, M.R., Verheijen, J., Hanemaaijer, R., Overgaard, J., 2010. Matrix metalloproteinase-9 measured in urine from bladder cancer patients is an independent prognostic marker of poor survival. Acta Oncol. 49, 1283-1287.

Ohnishi, Y., Tajima, S., Ishibashi, A., 2001. Coordinate expression of membrane type-matrix metalloproteinases-2 and 3 (MT2-MMP and MT3-MMP) and matrix metalloproteinase-2 (MMP-2) in primary and metastatic melanoma cells. Eur. J. Dermatol. 11, $420-423$.

Ohuchi, I., Imai, K., Fuji, Y., Sato, H., Seiki, M., Okada, Y., 1997. Membrane type 1 matrix metalloproteinase digests interstitial collagens and other extracellular matrix macromolecules. J. Biol. Chem. 272, 2446-2451.

Okada, A., Bellocq, J.P., Rouyer, N., Chenard, M.P., Rio, M.C., Chambon, P., Basset, P., 1995. Membrane-type matrix metalloproteinase (MT-MMP) gene is expressed in stromal cells of human colon, breast, and head and neck carcinomas. Proc. Natl. Acad. Sci. USA 92, $2730-2734$.

Okada, Y., 2009. Proteinases and matrix degradation. In: Firestein, G.S., Budd, R.C., Harris, E.D., Jr., McInnes, I.B., Ruddy, S., Sergent, J.S. (Eds.), Kelly's Texbook of Rheumatology, eighth ed. Saunders Elsevier, Philadelphia, PA, pp. 115-134.

Okamoto, Y., Satomura, K., Ohsuzu, F., Nakamura, H., Takeuchi, K., Yoshioka, M., 2001. Expression of matrix metalloproteinase 3 in experimental atherosclerotic plaques. J. Atheroscler. Thromb. 8, 50-64.

Okumura, Y., Sato, H., Seiki, M., Kido, H., 1997. Proteolytic activation of the precursor of membrane type 1 matrix metalloproteinase by human plasmin. A possible cell surface activator. FEBS Lett. 402, 181-184.

Oneda, B., Lods, N., Lottaz, D., Becker-Pauly, C., Stöcker, W., Pippin, J., Huguenin, M., Ambort, D., Marti, H.P., Sterchi, E.E., 2008. Metalloprotease meprin beta in rat kidney: glomerular localization and differential expression in glomerulonephritis. PLoS One 3, e2278.

Ongusaha, P.P., Kwak, J.C., Zwible, A.J., Macip, S., Higashiyama, S., Taniguchi, N., Fang, L., Lee, S.W., 2004. HB-EGF is a potent inducer of tumor growth and angiogenesis. Cancer Res. 64, 5283-5290.

Opdenakker, G., van den Steen, P.E., Dubois, B., Nelissen, I., Van Coillie, E., Masure, S., Proost, P., van Damme, J., 2001. Gelatinase B functions as regulator and effector in leukocyte biology. J. Leukoc. Biol. 69, 851-859.

Opdenakker, G., Nelissen, I., van Damme, J., 2003. Functional roles and therapeutic targeting of gelatinase B and chemokines in multiple sclerosis. Lancet Neurol. 2, 747-756.

Opdenakker, G., Dillen, C., Fiten, P., Martens, E., Van Aelst, I., Van den Steen, P.E., Nelissen, I., Starckx, S., Descamps, F.J., Hu, J., Piccard, H., Van Damme, J., Wormald, M.R., Rudd, P.M., Dwek, R.A., 2006. Remnant epitopes, autoimmunity and glycosylation. Biochim. Biophys. Acta $1760,610-615$.

Osman, I., Yee, H., Taneja, S.S., Levinson, B., Zeleniuch-Jacquotte, A., Chang, C., Nobert, C., Nanus, D.M., 2004. Neutral endopeptidase protein expression and prognosis in localized prostate cancer. Clin. Cancer Res. 10, 4096-4100.

Osteen, K.G., Igarshi, T.M., Bruner-Tran, K.L., 2003. Progesterone action in the human endometrium: induction of a unique tissue environment which limits matrix metalloproteinase (MMP) expression. Front. Biosci. 8, d78-d86.

Otterness, I.G., Bliven, M.L., Eskra, J.D., Koppele, J.M., Stukenbrok, H.A., Milici, A.J., 2000. Cartilage damage after intraarticular exposure to collagenase 3. Osteoarthritis Cartilage 8, 366-373.

Ottl, J., Gabriel, D., Murphy, G., Knäuper, V., Tominaga, Y., Nagase, H., 2000. Recognition and catabolism of synthetic heterotrimeric collagen peptides by matrix metalloproteinases. Chem. Biol. 7, 119-132.

Overall, C.M., Lopez-Otin, C., 2002. Strategies for MMP inhibition in cancer: innovations for the post-trial era. Nat. Rev. Cancer 2, 657-672.

Overall, C.M., Kleifeld, O., 2006a. Tumour microenvironment-opinion: validating matrix metalloproteinases as drug targets and anti-targets for cancer therapy. Nat. Rev. Cancer 6, 227-239.

Overall, C.M., Kleifeld, O., 2006b. Towards third generation matrix metalloproteinase inhibitors for cancer therapy. Br. J. Cancer 94, 941-946.

Overall, C.M., Butler, G.S., 2007. Protease yoga: extreme flexibility of a matrix metalloproteinase. Structure 15, 1159-1161.

Oxvig, C., 2001. Pregnancy-associated plasma protein-A (PAPP-A) cleaves insulin-like growth factor binding protein (IGFBP)-5 independent of IGF: implications for the mechanism of IGFBP-4 proteolysis by PAPP-A. FEBS Lett. 504, 36-40.

Oyamada, S., Bianchi, C., Takai, S., Chu, L.M., Sellke, F.W., 2011. Chymase inhibition reduces infarction and matrix metalloproteinase-9 activation and attenuates inflammation and fibrosis after acute myocardial ischemia/reperfusion. J. Pharmacol. Exp. Ther. 339, $143-151$.

Owen, C.A., Hu, Z., Lopez-Otin, C., Shapiro, S.D., 2004. Membrane-bound matrix metalloproteinase-8 on activated polymorphonuclear cells is a potent, tissue inhibitor of metalloproteinase-resistant collagenase and serpinase. J. Immunol. 172, 7791-7803.

Pachter, J.S., de Vries, H.E., Fabry, Z., 2003. The blood-brain barrier and its role in immune privilege in the central nervous system. J. Neuropathol. Exp. Neurol. 62, 593-604.

Paganetti, P.A., Caroni, P., Schwab, M.E., 1988. Glioblastoma infiltration into central nervous system tissue in vitro: involvement of a metalloprotease. J. Cell Biol. 107, 2281-2291.

Page-McCaw, A., Ewald, A.J., Werb, Z., 2007. Matrix metalloproteinases and the regulation of tissue remodelling. Nat. Rev. Mol. Cell Biol. 8, 221-233.

Pagenstecher, A., Stalder, A.K., Kincaid, C.L., Shapiro, S.D., Campbell, I.L., 1998. Differential expression of matrix metalloproteinase and tissue inhibitor of matrix metalloproteinase genes in the mouse central nervous system in normal and inflammatory states. Am. J. Pathol. 152, 729-741.

Pakozdi, A., Amin, M.A., Haas, C.S., Martinez, R.J., Haines 3rd, G.K., Santos, L.L., Morand, E.F., David, J.R., Koch, A.E., 2006. Macrophage migration inhibitory factor: a mediator of matrix metalloproteinase-2 production in rheumatoid arthritis. Arthritis Res. Ther. 8, R132.

Palosaari, H., Wahlgren, J., Larmas, M., Ronka, H., Sorsa, T., Salo, T., Tjäderhane, L., 2000. The expression of MMP-8 in human odontoblasts and dental pulp cells is down-regulated by TGF-beta1. J. Dent. Res. 79, 77-84.

Papandreou, C.N., Usmani, B., Geng, Y., Bogenrieder, T., Freeman, R., Wilk, S., Finstad, C.L., Reuter, V.E., Powell, C.T., Scheinberg, D., Magill, C., Scher, H.I., Albino, A.P., Nanus, D.M., 1998. Neutral endopeptidase 24.11 loss in metastatic human prostate cancer contributes to androgen-independent progression. Nat. Med. 4, 50-57.

Papaspyridonos, M., Smith, A., Burnand, K.G., Taylor, P., Padayachee, S., Suckling, K.E., James, C.H., Greaves, D.R., Patel, L., 2006. Novel candidate genes in unstable areas of human atherosclerotic plaques. Arterioscler. Thromb. Vasc. Biol. 26, 1837-1844.

Pardo, A., Barrios, R., Gaxiola, M., Segura-Valdez, L., Carrillo, G., Estrada, A., Meija, M., Selman, M., 2000. Increase of lung neutrophils in hypersensitivity pneumonitis is associated with lung fibrosis. Am. J. Respir. Crit. Care Med. 161, 1698-1704.

Park, H.I., Ni, J., Gerkema, F.E., Liu, D., Belozerov, V.E., Sang, Q.X., 2000. Identification and characterization of human endometase (matrix metalloproteinase26) from endometrial tumor. J. Biol. Chem. 275, 20540-20544.

Partridge, C.A., Phillips, P.G., Niedbala, M.J., Jeffrey, J.J., 1997. Localization and activation of type IV collagenase/gelatinase at endothelial focal contacts. Am. J. Physiol. 272, L813-L822.

Paschoalin, T., Carmona, A.K., Rodrigues, E.G., Oliveira, V., Monteiro, H.P., Juliano, M.A., Juliano, L., Travassos, L.R., 2007. Characterization of thimet oligopeptidase and neurolysin activities in B16F10-Nex2 tumor cells and their involvement in angiogenesis and tumor growth. Mol. Cancer $9,6-44$. 
Pascual, R.M., Peters, S.P., 2005. Airway remodeling contributes to the progressive loss of lung function in asthma: an overview. J. Allergy Clin. Immunol. $116,477-486$.

Patel, S., Sumitra, G., Koner, B.C., Saxena, A., 2011. Role of serum matrix metalloproteinase-2 and -9 to predict breast cancer progression. Clin. Biochem. 44, $869-872$.

Pathak, S., Goldofsky, E., Vivas, E.X., Bonagura, V.R., Vambutas, A., 2011. IL-1 $\beta$ is overexpressed and aberrantly regulated in corticosteroid nonresponders with autoimmune inner ear disease. J. Immunol. 186, 1870-1879.

Patterson, B.C., Sang, Q.A., 1997. Angiostatin-converting enzyme activities of human matrilysin (MMP-7) and gelatinase B/type IV collagenase (MMP-9). J. Biol. Chem. 272, 28823-28825.

Patterson, M.L., Atkinson, S.J., Knäuper, V., Murphy, G., 2001. Specific collagenolysis by gelatinase A, MMP-2, is determined by the hemopexin domain and not the fibronectin-like domain. FEBS Lett. 503, 158-162.

Pavlaki, M., Cao, J., Hymowitz, M., Chen, W.T., Bahou, W., Zucker, S., 2002. A conserved sequence within the propeptide domain of membrane type 1 matrix metalloproteinase is critical for function as an intramolecular chaperone. J. Biol. Chem. 277, 2740-2749.

Pei, D., Majmudar, G., Weiss, S.J., 1994. Hydrolytic inactivation of a breast carcinoma cell-derived serpin by human stromelysin-3. J. Biol. Chem. 269, 2584925855.

Pei, D., Weiss, S.J., 1995. Furin-dependent intracellular activation of the human stromelysin-3 zymogen. Nature 375, 244-247.

Pei, D., Weiss, S.J., 1996. Transmembrane-deletion mutants of the membrane-type matrix metalloproteinase-1 process progelatinase A and express intrinsic matrix-degrading activity. J. Biol. Chem. 271, 9135-9140.

Pei, D., 1999a. Identification and characterization of the fifth membrane-type matrix metalloproteinase MT5-MMP. J. Biol. Chem. 274, 8925-8932.

Pei, D., 1999b. Leukolysin/MMP25/MT6-MMP: a novel matrix metalloproteinase specifically expressed in the leukocyte lineage. Cell Res. 9, 291-303.

Pelletier, M., Roberge, C.J., Gauthier, M., Vandal, K., Tessier, P.A., Girard, D., 2001. Activation of human neutrophils in vitro and dieldrin-induced neutrophilic inflammation in vivo. J. Leukoc. Biol. 70, 367-373.

Pendas, A.M., Knäuper, V., Puente, X.S., Llano, E., Mattei, M.G., Apte, S., Murphy, G., Lopez-Otin, C., 1997. Identification and characterization of a novel human matrix metalloproteinase with unique structural characteristics, chromosomal location, and tissue distribution. J. Biol. Chem. 272, $4281-4286$.

Pendas, A.M., Uria, J.A., Jimenez, M.G., Balbin, M., Freije, J.P., Lopez-Otin, C., 2000. An overview of collagenase-3 expression in malignant tumors and analysis of its potential value as a target in antitumor therapies. Clin. Chim. Acta 291, 137-155.

Petrow, P.K., Wernicke, D., Schulze Westhoff, C., Hummel, K.M., Brauer, R., Kriegsmann, J., Gromnica-Ihle, E., Gay, R.E., Gay, S., 2002. Characterisation of the cell type specificity of collagenase 3 mRNA expression in comparison with membrane type 1 matrix metalloproteinase and gelatinase A in the synovial membrane in rheumatoid arthritis. Ann. Rheum. Dis. 61, 391-397.

Pilcher, B.K., Dumin, J.A., Sudbeck, B.D., Krane, S.M., Welgus, H.G., Parks, W.C., 1997. The activity of collagenase-1 is required for keratinocyte migration on a type I collagen matrix. J. Cell Biol. 137, 1445-1457.

Pirard, B., 2007. Insight into the structural determinants for selective inhibition of matrix metalloproteinases. Drug Discov. Today 12, 640-646.

Plaisier, M., Kapiteijn, K., Koolwijk, P., Fijten, C., Hanemaaijer, R., Grimbergen, J.M., Mulder-Stapel, A., Quax, P.H., Helmerhorst, F.M., van Hinsbergh, V.W., 2004. Involvement of membrane-type matrix metalloproteinases (MT-MMPs) in capillary tube formation by human endometrial microvascular endothelial cells: role of MT3-MMP. Clin. Endocrinol. Metab. 89, 5828-5836.

Plumb, J., McQuaid, S., Cross, A.K., Surr, J., Haddock, G., Bunning, R.A., Woodroofe, M.N., 2006. Upregulation of ADAM-17 expression in active lesions in multiple sclerosis. Mult. Scler. 12, 375-385.

Polette, M., Nawrocki, B., Gilles, C., Sato, H., Seiki, M., Tournier, J.M., Birembaut, P., 1996. MT-MMP expression and localisation in human lung and breast cancers. Virch. Arch. 428, 29-35.

Polette, M., Birembaut, P., 1998. Membrane-type metalloproteinases in tumor invasion. Int. J. Biochem. Cell Biol. 30, 1195-1202.

Pollio, G., Hoozemans, J.J., Andersen, C.A., Roncarati, R., Rosi, M.C., van Haastert, E.S., Seredenina, T., Diamanti, D., Gotta, S., Fiorentini, A., Magnoni, L., Raggiaschi, R., Rozemuller, A.J., Casamenti, F., Caricasole, A., Terstappen, G.C., 2008. Increased expression of the oligopeptidase THOP1 is a neuroprotective response to Abeta toxicity. Neurobiol. Dis. 31, 145-158.

Polyak, K., Weinberg, R.A., 2009. Transitions between epithelial and mesenchymal states: acquisition of malignant and stem cell traits. Nat. Rev. Cancer 9, $265-273$.

Poola, I., DeWitty, R.L., Marshalleck, J.J., Bhatnagar, R., Abraham, J., Leffall, L.D., 2005. Identification of MMP-1 as a putative breast cancer predictive marker by global gene expression analysis. Nat. Med. 11, 481-483.

Porte, H., Chastre, E., Prevot, S., Nordlinger, B., Empereur, S., Basset, P., Chambon, P., Gespach, C., 1995. Neoplastic progression of human colorectal cancer is associated with overexpression of the stromelysin-3 and BM-40/SPARC genes. Int. J. Cancer 64, 70-75.

Porter, S., Clark, I.M., Kevorkian, L., Edwards, D.R., 2005. The ADAMTS metalloproteinases. Biochem. J. 386, $15-27$.

Posthumus, M., Collins, M., van der Merwe, L., O’Cuinneagain, D., van der Merwe, W., Ribbans, W.J., Schwellnus, M.P., Raleigh, S.M., in press. Matrix metalloproteinase genes on chromosome 11q22 and the risk of anterior cruciate ligament (ACL) rupture. Scand. J. Med. Sci. Sports.

Powell, W.C., Fingleton, B., Wilson, C.L., Boothby, M., Matrisian, L.M., 1999. The metalloproteinase matrilysin proteolytically generates active soluble Fas ligand and potentiates epithelial cell apoptosis. Curr. Biol. 9, 1441-1447.

Power, C., O'Connor, C.M., MacFarlane, D., O’Mahoney, S., Gaffney, K., Hayes, J., Fitzgerald, M.X., 1994. Neutrophil collagenase in sputum from patients with cystic fibrosis. Am. J. Respir. Crit. Care Med. 150, 818-822.

Pratta, M.A., Yao, W., Decicco, C., Tortorella, M.D., Liu, R.Q., Copeland, R.A., Magolda, R., Newton, R.C., Trzaskos, J.M., Arner, E.C., 2003. Aggrecan protects cartilage collagen from proteolytic cleavage. J. Biol. Chem. 278, 45539-45545.

Prikk, K., Maisi, P., Pirilä, E., Sepper, R., Salo, T., Wahlgren, J., Sorsa, T., 2001. In vivo collagenase-2 (MMP-8) expression by human bronchial epithelial cells and monocytes/macrophages in bronchiectasis. J. Pathol. 194, 232-238.

Prikk, K., Maisi, P., Pirilä, E., Reintam, M.A., Salo, T., Sorsa, T., Sepper, R., 2002. Airway obstruction correlates with collagenase-2 (MMP-8) expression and activation in bronchial asthma. Lab. Invest. 82, 1535-1545.

Prockop, D.J., Kivirikko, K.I., 1995. Collagens: molecular biology, diseases, and potentials for therapy. Annu. Rev. Biochem. 64, 403-434.

Puente, X.S., Pendas, A.M., Llano, E., Velasco, G., Lopez Otin, C., 1996. Molecular cloning of a novel membrane-type matrix metalloproteinase from a human breast carcinoma. Cancer Res. 56, 944-949.

Pyo, R., Lee, J.K., Shipley, J.M., Curci, J.A., Mao, D., Ziporin, S.J., Ennis, T.L., Shapiro, S.D., Senior, R.M., Thompson, R.W., 2000. Targeted gene disruption of matrix metalloproteinase-9 (gelatine B) suppresses development of experimental abdominal aortic aneurysms. J. Clin. Invest. 105, 1641-1649.

Qu, P., Du, H., Wang, X., Yan, C., 2009. Matrix metalloproteinase 12 overexpression in lung epithelial cells plays a key role in emphysema to lung bronchioalveolar adenocarcinoma transition. Cancer Res. 69, 7252-7261.

Qu, P., Yan, C., Du, H., 2011. Matrix metalloproteinase 12 overexpression in myeloid lineage cells plays a key role in modulating myelopoiesis, immune suppression, and lung tumorigenesis. Blood 117, 4476-4489.

Quantin, B., Murphy, G., Breathnach, R., 1989. Pump-1 cDNA codes for a protein with characteristics similar to those of classical collagenase family members. Biochemistry 28, 5327-5334.

Quaschning, T., 2005. Vasopeptidase inhibition for blood pressure control: emerging experience. Curr. Pharm. Des. 11, $3293-3299$.

Quillard, T., Tesmenitsky, Y., Croce, K., Travers, R., Shvartz, E., Koskinas, K.C., Sukhova, G., Aikawa, E., Aikawa, M., Libby, P., 2011. Selective inhibition of matrix metalloproteinase-13 increases collagen content of established mouse atheromas. Arterioscler. Thromb. Vasc. Biol. 31, 2464-2472.

Ra, H.J., Harju-Baker, S., Zhang, F., Linhardt, R.J., Wilson, C.L., Parks, W.C., 2009. Control of promatrilysin (MMP7) activation and substrate-specific activity by sulfated glycosaminoglycans. J. Biol. Chem. 284, 27924-27932.

Radichev, I.A., Remacle, A.G., Shiryaev, S.A., Purves, A.N., Johnson, S.L., Pellecchia, M., Strongin, A.Y., 2010. Biochemical characterization of the cellular glycosylphosphatidylinositol-linked membrane type-6 matrix metalloproteinase. J. Biol. Chem. 285, 16076-16086. 
Radjabi, A.R., Sawada, K., Jagadeeswaran, S., Eichbichler, A., Kenny, H.A., Montag, A., Bruno, K., Lengyel, E., 2008. Thrombin induces tumor invasion through the induction and association of matrix metalloproteinase-9 and beta1-integrin on the cell surface. J. Biol. Chem. $283,2822-2834$.

Ragin, A.B., Wu, Y., Ochs, R., Scheidegger, R., Cohen, B.A., McArthur, J.C., Epstein, L.G., Conant, K., 2009. Serum matrix metalloproteinase levels correlate with brain injury in human immunodeficiency virus infection. J. Neurovirol. 15, 275-281.

Ragin, A.B., Wu, Y., Ochs, R., Du, H., Epstein, L.G., Conant, K., McArthur, J.C., 2011. Marked relationship between matrix metalloproteinase 7 and brain atrophy in HIV infection. J. Neurovirol. 17, 153-158.

Rajah, R., Nunn, S.E., Herrick, D.J., Grunstein, M.M., Cohen, P., 1996. Leukotriene D4 induces MMP-1, which functions as an IGFBP protease in human airway smooth muscle cells. Am. J. Physiol. 271, L1014-L1022.

Rajoria, S., Suriano, R., George, A., Shanmugam, A., Schantz, S.P., Geliebter, J., Tiwari, R.K., 2011. Estrogen induced metastatic modulators MMP-2 and MMP-9 are targets of 3,3'-diindolylmethane in thyroid cancer. PLoS One 6, e15879.

Ram, M., Sherer, Y., Shoenfeld, Y., 2006. Matrix metalloproteinase-9 and autoimmune diseases. J. Clin. Immunol. 26, 299-307.

Ramirez, C.L., Cadinanos, J., Varela, I., Freije, J.M., Lopez-Otin, C., 2007. Human progeroid syndromes, aging and cancer: new genetic and epigenetic insights into old questions. Cell. Mol. Life Sci. 64, 155-170.

Ramos-Fernandez, M., Bellolio, M.F., Stead, L.G., 2011. Matrix metalloproteinase-9 as a marker for acute ischemic stroke: a systematic review. J. Stroke Cerebrovasc. Dis. 20, 47-54

Ranasinghe, H.S., Williams, C.E., Christophidis, L.J., Mitchell, M.D., Fraser, M., Scheepens, A., 2009. Proteolytic activity during cortical development is distinct from that involved in hypoxic ischemic injury. Neuroscience 158, 732-744.

Ranogajec, I., Jakić-Razumović, J., Puzović, V., Gabrilovac, J., in press. Prognostic value of matrix metalloproteinase-2 (MMP-2), matrix metalloproteinase-9 (MMP-9) and aminopeptidase N/CD13 in breast cancer patients. Med. Oncol.

Ratjen, F., Hartog, C.M., Paul, K., Wermelt, J., Braun, J., 2002. Matrix metalloproteases in BAL fluid of patients with cystic fibrosis and their modulation by treatment with dornase alpha. Thorax 57, 930-934.

Ratnikov, B.I., Rozanov, D.V., Postnova, T.I., Baciu, P.G., Zhang, H., DiScipio, R.G., Chestukhina, G.G., Smith, J.W., Deryugina, E.I., Strongin, A.Y., 2002. An alternative processing of integrin alpha(v) subunit in tumor cells by membrane type-1 matrix metalloproteinase. J. Biol. Chem. 277, $7377-7385$

Raza, S.L., Nehring, L.C., Shapiro, S.D., Cornelius, L.A., 2000. Proteinase-activated receptor-1 regulation of macrophage elastase (MMP-12) secretion by serine proteinases. J. Biol. Chem. 275, 41243-41250.

Reboul, P., Pelletier, J.P., Tardif, G., Cloutier, J.M., Martel-Pelletier, J., 1996. The new collagenase, collagenase-3, is expressed and synthesized by human chondrocytes but not by synoviocytes: a role in osteoarthritis. J. Clin. Invest. 97, 2011-2019.

Red Eagle, A.R., Hanson, R.L., Jiang, W., Han, X., Matters, G.L., Imperatore, G., Knowler, W.C., Bond, J.S., 2005. Meprin beta metalloprotease gene polymorphisms associated with diabetic nephropathy in the Pima Indians. Hum. Genet. 118, 12-22.

Redman, C.M., Russo, D., Lee, S., 1999. Kell, Kx and the McLeod syndrome. Baillieres Best Pract. Res. Clin. Haematol. 12, 621-635.

Redondo-Muñoz, J., Ugarte-Berzal, E., Garcia-Marco, J.A., del Cerro, M.H., Van den Steen, P.E., Opdenakker, G., Terol, M.J., Garcia-Pardo, A., 2008. Alpha4beta1 integrin and 190-kDa CD44v constitute a cell surface docking complex for gelatinase B/MMP-9 in chronic leukemic but not in normal B cells. Blood 112, 169-178.

Reeps, C., Pelisek, J., Seidl, S., Schuster, T., Zimmermann, A., Kuehnl, A., Eckstein, H.H., 2009. Inflammatory infiltrates and neovessels are relevant sources of MMPs in abdominal aortic aneurysm wall. Pathobiology 76, 243-252.

Reichel, C.A., Rehberg, M., Bihari, P., Moser, C.M., Linder, S., Khandoga, A., Krombach, F., 2008. Gelatinases mediate neutrophil recruitment in vivo: evidence for stimulus specificity and a critical role in collagen IV remodeling. J. Leukoc. Biol. 83, 864-874.

Reinemer, P., Grams, F., Huber, R., Kleine, T., Schnierer, S., Piper, M., Tschesche, H., Bode, W., 1994. Structural implications for the role of the N terminus in the "superactivation" of collagenases. A crystallographic study. FEBS Lett. 338, 227-233.

Reiss, K., Maretzky, T., Ludwig, A., Tousseyn, T., de Strooper, B., Hartmann, D., Saftig, P., 2005. ADAM10 cleavage of N-cadherin and regulation of cell-cell adhesion and beta-catenin nuclear signalling. EMBO J. 24, 742-752.

Reno, F., Sabbatini, M., Stella, M., Magliacani, G., Cannas, M., 2005. Effect of in vitro mechanical compression on epilysin (matrix metalloproteinase-28) expression in hypertrophic scars. Wound Repair Regen. 13, 255-261.

Ribeiro, A.S., Albergaria, A., Sousa, B., Correia, A.L., Bracke, M., Seruca, R., Schmitt, F.C., Paredes, J., 2010. Extracellular cleavage and shedding of P-cadherin: a mechanism underlying the invasive behaviour of breast cancer cells. Oncogene 29, 392-402.

Riddick, A.C., Shukla, C.J., Pennington, C.J., Bass, R., Nuttall, R.K., Hogan, A., Sethia, U.K., Ellis, V., Collins, A.T., Maitland, N.J., Ball, R.Y., Edwards, D.R., 2005. Identification of degradome components associated with prostate cancer progression by expression analysis of human prostatic tissues. Br. J. Cancer 92 , 2171-2180.

Riggins, K.S., Mernaugh, G., Su, Y., Quaranta, V., Koshikawa, N., Seiki, M., Pozzi, A., Zent, R., 2010. MT1-MMP-mediated basement membrane remodeling modulates renal development. Exp. Cell Res. 316, 2993-3005.

Rio, M.C., 2002. Stromelysin-3, a Particular Member of the Matrix Metalloproteinase Family, vol. 4, Kluwer Academic ed.. Kluwer Academic Publisher, Dordrecht.

Rio, M.C., 2005. From a unique cell to metastasis is a long way to go: clues to stromelysin-3 participation. Biochimie 87, 299-306.

Rivera, S., Tremblay, E., Timsit, S., Canals, O., Ben-Ari, Y., Khrestchatisky, M., 1997. Tissue inhibitor of metalloproteinases-1 (TIMP-1) is differentially induced in neurons and astrocytes after seizures: evidence for developmental, immediate early gene, and lesion response. J. Neurosci. $17,4223-4235$.

Rizki, A., Weaver, V.M., Lee, S.Y., Rozenberg, G.I., Chin, K., Myers, C.A., Bascom, J.L., Mott, J.D., Semeiks, J.R., Grate, L.R., Mian, I.S., Borowsky, A.D., Jensen, R.A., Idowu, M.O., Chen, F., Chen, D.J., Petersen, O.W., Gray, J.W., Bissell, M.J., 2008. A human breast cell model of preinvasive to invasive transition. Cancer Res. 68, 1378-1387

Rocks, N., Paulissen, G., Quesada-Calvo, F., Munaut, C., Gonzalez, M.L., Gueders, M., Hacha, J., Gilles, C., Foidart, J.M., Noel, A., Cataldo, D.D., 2008. ADAMTS-1 metalloproteinase promotes tumor development through the induction of a stromal reaction in vivo. Cancer Res. 68, 9541-9550.

Rodriguez, J.A., Orbe, J., Martinez de Lizarrondo, S., Calvayrac, O., Rodriguez, C., Martinez-Gonzalez, J., Paramo, J.A., 2008. Metalloproteinases and atherothrombosis: MMP-10 mediates vascular remodeling promoted by inflammatory stimuli. Front. Biosci. 13, $2916-2921$.

Rodriguez-Feo, J.A., Hellings, W.E., Moll, F.L., De Vries, J.P., van Middelaar, B.J., Algra, A., Sluijter, J., Velema, E., van den Broek, T., Sessa, W.C., De Kleijn, D.P., Pasterkamp, G., 2008. Caveolin-1 influences vascular protease activity and is a potential stabilizing factor in human atherosclerotic disease. PLoS One 3, e2612.

Rojiani, M.V., Alidina, J., Esposito, N., Rojiani, A.M., 2010. Expression of MMP-2 correlates with increased angiogenesis in CNS metastasis of lung carcinoma. Int. J. Clin. Exp. Pathol. 3, 775-781.

Romanic, A.M., Burns-Kurtis, C.L., Ao, Z., Arleth, A.J., Ohlstein, E.H., 2001. Upregulated expression of human membrane type-5 matrix metalloproteinase in kidneys from diabetic patients. Am. J. Physiol. Renal Physiol. 281, F309-F317.

Roques, B.P., Fournié-Zaluski, M.C., Soroca, E., Lecomte, J.M., Malfroy, B., Llorens, C., Schwartz, J.C., 1980. The enkephalinase inhibitor thiorphan shows antinociceptive activity in mice. Nature 288, 286-288.

Rosas, I.O., Richards, T.J., Konishi, K., Zhang, Y., Gibson, K., Lokshin, A.E., Lindell, K.O., Cisneros, J., Macdonald, S.D., Pardo, A., Sciurba, F., Dauber, J., Selman, M., Gochuico, B.R., Kaminski, N., 2008. MMP1 and MMP7 as potential peripheral blood biomarkers in idiopathic pulmonary fibrosis. PLoS Med. 5, e93.

Rosell, A., Alvarez-Sabin, J., Arenillas, J.F., Rovira, A., Delgado, P., Fernandez-Cadenas, I., Penalba, A., Molina, C.A., Montaner, J., 2005. A matrix metalloproteinase protein array reveals a strong relation between MMP-9 and MMP-13 with diffusion-weighted image lesion increase in human stroke. Stroke 36, 1415-1420.

Rosenberg, G.A., 2005. Matrix metalloproteinases biomarkers in multiple sclerosis. Lancet 365, 1291-1293.

Rosenblum, G., Van den Steen, P.E., Cohen, S.R., Grossmann, J.G., Frenkel, J., Sertchook, R., Slack, N., Strange, R.W., Opdenakker, G., Sagi, I., 2007a. Insights into the structure and domain flexibility of full-length pro-matrix metalloproteinase-9/gelatinase B. Structure 15, 1227-1236. 
Rosenblum, G., Meroueh, S., Toth, M., Fisher, J.F., Fridman, R., Mobashery, S., Sagi, I., 2007b. Molecular structures and dynamics of the stepwise activation mechanism of a matrix metalloproteinase zymogen: challenging the cysteine switch dogma. J. Am. Chem. Soc. 129, $13566-13574$.

Ross, H.H., Fillmore, H.L., 2007. Identification of a novel human MT5-MMP transcript variant in multipotent NT2 cells. FEBS Lett. 581, $5923-5928$.

Rouyer, N., Wolf, C., Chenard, M.P., Rio, M.C., Chambon, P., Bellocq, J.P., Basset, P., 1994. Stromelysin-3 gene expression in human cancer: an overview. Invasion Metastasis 14, 269-275.

Roy, R., Wewer, U.M., Zurakowski, D., Pories, S.E., Moses, M.A., 2004. ADAM 12 cleaves extracellular matrix proteins and correlates with cancer status and stage. J. Biol. Chem. 279, 51323-51330.

Rozanov, D.V., Deryugina, E.I., Ratnikov, B.I., Monosov, E.Z., Marchenko, G.N., Quigley, J.P., Strongin, A.Y., 2001. Mutation analysis of membrane type-1 matrix metalloproteinase (MT1-MMP). The role of the cytoplasmic tail Cys(574), the active site Glu(240), and furin cleavage motifs in oligomerization, processing, and self-proteolysis of MT1-MMP expressed in breast carcinoma cells. J. Biol. Chem. 276, 25705-25714.

Rozanov, D., Hahn-Dantona, E., Strickland, D.K., Strongin, A.Y., 2004. The low density lipoprotein receptor-related protein LRP is regulated by membrane type-1 matrix metalloproteinase (MT1-MMP) proteolysis in malignant cells. J. Biol. Chem. 279, 4260-4268.

Rugarli, E.I., Langer, T., 2006. Translating m-AAA protease function in mitochondria to hereditary spastic paraplegia. Trends Mol. Med. 12, 262-269.

Ruiz, V., Ordoñez, R.M., Berumen, J., Ramirez, R., Uhal, B., Becerril, C., Pardo, A., Selman, M., 2003. Unbalanced collagenase/TIMP-1 expression and epithelial apoptosis in experimental lung fibrosis. Am. J. Physiol. Lung Cell. Mol. Physiol. 285, L1026-L1036.

Rundhaug, E.J., 2003. Matrix metalloproteinases, angiogenesis, and cancer. Clin. Cancer Res. 9, 551-554.

Russell, R.E., Thorley, A., Culpitt, S.V., Dodd, S., Donnelly, L.E., Demattos, C., Fitzgerald, M., Barnes, P.J., 2002. Alveolar macrophage-mediated elastolysis: roles of matrix metalloproteinases, cysteine, and serine proteases. Am. J. Physiol. Lung Cell. Mol. Physiol. 283, L867-L873.

Saarialho-Kere, U., Kerkela, E., Jeskanen, L., Hasan, T., Pierce, R., Starcher, B., Raudasoja, R., Ranki, A., Oikarinen, A., Vaalamo, M., 1999. Accumulation of matrilysin (MMP-7) and macrophage metalloelastase (MMP-12) in actinic damage. J. Invest. Dermatol. 113, 664-672.

Saarialho-Kere, U., Kerkela, E., Jahkola, T., Suomela, S., Keski-Oja, J., Lohi, J., 2002. Epilysin (MMP-28) expression is associated with cell proliferation during epithelial repair. J. Invest. Dermatol. 119, 14-21.

Sabatine, M.S., Ploughman, L., Simonsen, K.L., Iakoubova, O.A., Kirchgessner, T.G., Ranade, K., Tsuchihashi, Z., Zerba, K.E., Long, D.U., Tong, C.H., Packard, C.J., Pfeffer, M.A., Devlin, J.J., Shepherd, J., Campos, H., Sacks, F.M., Braunwald, E., 2008. Association between ADAMTS1 matrix metalloproteinase gene variation, coronary heart disease, and benefit of statin therapy. Arterioscler. Thromb. Vasc. Biol. 28, 562-567.

Sabbagh, Y., Jones, A.O., Tenenhouse, H.S., 2000. PHEXdb, a locus-specific database for mutations causing X-linked hypophosphatemia. Hum. Mutat. 16, 1-6.

Sabeh, F., Li, X.Y., Saunders, T.L., Rowe, R.G., Weiss, S.J., 2009. Secreted versus membrane-anchored collagenases: relative roles in fibroblast-dependent collagenolysis and invasion. J. Biol. Chem. 28, 23001-23011.

Saber, T., Veale, D.J., Balogh, E., McCormick, J., Nicanultaigh, S., Connolly, M., Fearon, U., 2011. Toll-like receptor 2 induced angiogenesis and invasion is mediated through the Tie2 signalling pathway in rheumatoid arthritis. PLoS One 6, e23540.

Sacre, S.M., Andreakos, E., Kiriakidis, S., Amjadi, P., Lundberg, A., Giddins, G., Feldmann, M., Brennan, F., Foxwell, B.M., 2007. The Toll-like receptor adaptor proteins MyD88 and Mal/TIRAP contribute to the inflammatory and destructive processes in a human model of rheumatoid arthritis. Am. J. Pathol. 170, $518-525$.

Sadler, J.E., Mannucci, P.M., Berntorp, E., Bochkov, N., Boulyjenkov, V., Ginsburg, D., Meyer, D., Peake, I., Rodeghiero, F., Srivastava, A., 2000. Impact, diagnosis and treatment of von Willebrand disease. Thromb. Haemost. 84, 160-174.

Sadowski, T., Dietrich, S., Koschinsky, F., Sedlacek, R., 2003. Matrix metalloproteinase 19 regulates insulin-like growth factor-mediated proliferation, migration, and adhesion in human keratinocytes through proteolysis of insulin-like growth factor binding protein-3. Mol. Biol. Cell 14, 4569-4580.

Sadowski, T., Dietrich, S., Koschinsky, F., Ludwig, A., Proksch, E., Titz, B., Sedlacek, R., 2005. Matrix metalloproteinase 19 processes the laminin 5 gamma 2 chain and induces epithelial cell migration. Cell. Mol. Life Sci. 62, 870-880.

Saftig, P., Hartmann, D., 2005. ADAM10 A major membrane protein ectodomain sheddase involved in regulated intramembrane proteolysis. In: Hooper, N.M., Lendeckel, U. (Eds.), The Adam Family of Proteases. Springer, pp. 85-121.

Sakakibara, M., Koizumi, S., Saikawa, Y., Wada, H., Ichihara, T., Sato, H., Horita, S., Mugishima, H., Kaneko, Y., Koike, K., 1999. Membrane-type matrix metalloproteinase-1 expression and activation of gelatinase A as prognostic markers in advanced pediatric neuroblastoma. Cancer 85, $231-239$.

Salinas, S., Proukakis, C., Crosby, A., Warner, T.T., 2008. Hereditary spastic paraplegia: clinical features and pathogenetic mechanisms. Lancet Neurol. 7, 1127-1138.

Salmela, M.T., Pender, S.L., Karjalainen-Lindsberg, M.L., Puolakkainen, P., Macdonald, T.T., Saarialho-Kere, U., 2004. Collagenase-1 (MMP-1), matrilysin-1 (MMP-7), and stromelysin-2 (MMP-10) are expressed by migrating enterocytes during intestinal wound healing. Scand. J. Gastroenterol. 39, 10951104.

Salsas-Escat, R., Nerenberg, P.S., Stultz, C.M., 2010. Cleavage site specificity and conformational selection in type I collagen degradation. Biochemistry 49 4147-4158.

Salter, R.C., Ashlin, T.G., Kwan, A.P., Ramji, D.P., 2010. ADAMTS proteases: key roles in atherosclerosis? J. Mol. Med. 88, $1203-1211$.

Sanchez, A.J., Puerta, C., Ballester, S., Gonzalez, P., Arriaga, A., Garcia-Merino, A., 2005. Rolipram impairs NF-kappaB activity and MMP-9 expression in experimental autoimmune encephalomyelitis. J. Neuroimmunol. 168, 13-20.

Sandler, N.G., Mentink-Kane, M.M., Cheever, A.W., Wynn, T.A., 2003. Global gene expression profiles during acute pathogen-induced pulmonary inflammation reveal divergent roles for Th1 and Th2 responses in tissue repair. J. Immunol. 171, 3655-3667.

Sands, M.F., Ohtake, P.J., Mahajan, S.D., Takyar, S.S., Aalinkeel, R., Fang, Y.V., Blume, J.W., Mullan, B.A., Sykes, D.E., Lachina, S., Knight, P.R., Schwartz, S.A., 2009. Tissue inhibitor of metalloproteinase-1 modulates allergic lung inflammation in murine asthma. Clin. Immunol. 130, 186-198.

Sands, M.F., in press. Localization of matrix metalloproteinase (MMP)-9 in lung tissue of a murine model of allergic asthma. Immunol. Invest.

Sandy, J.D., Neame, P.J., Boynton, R.E., Flannery, C.R., 1991. Catabolism of aggrecan in cartilage explants. J. Biol. Chem. $266,8683-8685$.

Sanjmyatav, J., Steiner, T., Wunderlich, H., Diegmann, J., Gajda, M., Junker, K., 2011. A specific gene expression signature characterizes metastatic potential in clear cell renal cell carcinoma. J. Urol. 186, 289-294.

Sans-Fons, M.G., Sole, S., Sanfeliu, C., Planas, A.M., 2010. Matrix metalloproteinase-9 and cell division in neuroblastoma cells and bone marrow macrophages. Am. J. Pathol. 177, 2870-2885.

Sariahmetoglu, M., Crawford, B.D., Leon, H., Sawicka, J., Li, L., Ballermann, B.J., Holmes, C., Berthiaume, L.G., Holt, A., Sawicki, G., Schulz, R., 2007. Regulation of matrix metalloproteinase-2 (MMP-2) activity by phosphorylation. FASEB J. 21, 2486-2495.

Sato, H., Takino, T., Okada, Y., Cao, J., Shinagawa, A., Yamamoto, E., Seiki, M., 1994. A matrix metalloproteinase expressed on the surface of invasive tumour cells. Nature 370, 61-65.

Sato, H., Seiki, M., 1996. Membrane-type matrix metalloproteinases (MTMMPs) in tumor metastasis. J. Biochem. 119, $209-215$.

Sato, H., Kinoshita, T., Takino, T., Nakayama, K., Seiki, M., 1996. Activation of a recombinant membrane type 1-matrix metalloproteinase (MT1-MMP) by furin and its interaction with tissue inhibitor of metalloproteinases (TIMP)-2. FEBS Lett. 393, 101-104.

Sato, H., Takino, T., 2010. Coordinate action of membrane-type matrix metalloproteinase-1 (MT1-MMP) and MMP-2 enhances pericellular proteolysis and invasion. Cancer Sci. 101, 843-847.

Saus, J., Quinones, S., Otani, Y., Nagase, H., Harris Jr., E.D., Kurkinen, M., 1988. The complete primary structure of human matrix metalloproteinase-3. Identity with stromelysin. J. Biol. Chem. 263, 6742-6745.

Savio, M.D.A., Halpern, A.R., Wu, Y., Li, W., Sypek, J., Quinet, E.M., 2008. MMP-12 inhibitor reduces severity of ANGII-induced aortic abdominal aneurysms in apoE-/-. Circulation 118, S473.

Schechter, I., Berger, A., 1967. On the size of the active site in proteases. I. Papain. Biochem. Biophys. Res. Commun. 27, 157-162.

Schenk, S., Quaranta, V., 2003. Tales from the cryptic sites of the extracellular matrix. Trends Cell Biol. 13, $366-375$. 
Scherf, D.B., Dally, H., Müller, P., Werle-Schneider, G., Jäger, B., Edler, L., Tuengerthal, S., Fischer, J.R., Drings, P., Bartsch, H., Risch, A., 2010. Single nucleotide polymorphisms in matrix metalloproteinase genes and lung cancer chemotherapy response and prognosis. Eur. Respir. J. 35, 381-390.

Schlomann, U., Wildeboer, D., Webster, A., Antropova, O., Zeuschner, D., Knight, C.G., Docherty, A.J., Lambert, M., Skelton, L., Jockusch, H., Bartsch, J.W., 2002. The metalloprotease disintegrin ADAM8. Processing by autocatalysis is required for proteolytic activity and cell adhesion. J. Biol. Chem. 277, 4821048219.

Schneider, P., Holler, N., Bodmer, J.L., Hahne, M., Frei, K., Fontana, A., Tschopp, J., 1998. Conversion of membrane-bound Fas(CD95) ligand to its soluble form is associated with downregulation of its proapoptotic activity and loss of liver toxicity. J. Exp. Med. 187, 1205-1213.

Schneider, F., Sukhova, G.K., Aikawa, M., Canner, J., Gerdes, N., Tanq, S.M., Shi, G.P., Apte, S.S., Libby, P., 2008. Matrix metalloproteinase-14 deficiency in bonemarrow-derived cells promotes collagen accumulation in mouse atherosclerotic plaques. Circulation 117, 931-939.

Schönbeck, U., Mach, F., Libby, P., 1998. Generation of biologically active IL-1 beta by matrix metalloproteinases: a novel caspase-1-independent pathway of IL-1 beta processing. J. Immunol. 161, 3340-3346.

Schönbeck, U., Mach, F., Sukhova, G.K., Atkinson, E., Levesque, E., Herman, M., Graber, P., Basset, P., Libby, P., 1999. Expression of stromelysin-3 in atherosclerotic lesions: regulation via CD40-CD40 ligand signaling in vitro and in vivo. J. Exp. Med. 189, 843-853.

Schoser, B.G., Blottner, D., Stuerenburg, H.J., 2002. Matrix metalloproteinases in inflammatory myopathies: enhanced immunoreactivity near atrophic myofibers. Acta Neurol. Scand. 105, 309-313.

Schulz, B., Pruessmeyer, J., Maretzky, T., Ludwig, A., Blobel, C.P., Saftig, P., Reiss, K., 2008. ADAM10 regulates endothelial permeability and T-cell transmigration by proteolysis of vascular endothelial cadherin. Circ. Res. 102, 1192-1201.

Schuppan, D., Schmid, M., Somasundaram, R., Ackermann, R., Ruehl, M., Nakamura, T., Riecken, E.O., 1998. Collagens in the liver extracellular matrix bind hepatocyte growth factor. Gastroenterology 114, 139-152.

Seals, D.F., Courtneidge, S.A., 2003. The ADAMs family of metalloproteases: multidomain proteins with multiple functions. Genes Dev. 17, 7-30.

Secchiero, P., Gonelli, A., Corallini, F., Ceconi, C., Ferrari, R., Zauli, G., 2010. Metalloproteinase-2 cleaves in vitro recombinant TRAIL: potential implications for the decreased serum levels of TRAIL after acute myocardial infarction. Atherosclerosis 211, 333-346.

Sedlacek, R., Mauch, S., Kolb, B., Schätzlein, C., Eibel, H., Peter, H.H., Schmitt, J., Krawinkel, U., 1998. Matrix metalloproteinase MMP-19 (RASI-1) is expressed on the surface of activated peripheral blood mononuclear cells and is detected as an autoantigen in rheumatoid arthritis. Immunobiology $198,408-423$

Seftor, R.E., Seftor, E.A., Koshikawa, N., Meltzer, P.S., Gardner, L.M., Bilban, M., Stetler-Stevenson, W.G., Quaranta, V., Hendrix, M.J., 2001. Cooperative interactions of laminin 5 gamma2 chain, matrix metalloproteinase-2, and membrane type-1-matrix/metalloproteinase are required for mimicry of embryonic vasculogenesis by aggressive melanoma. Cancer Res. 61, 6322-6327.

Seiki, M., 1999. Membrane-type matrix metalloproteinase. APMIS 107 (1), 137-143.

Seiki, M., 2003. Membrane-type 1 matrix metalloproteinase: a key enzyme for tumor invasion. Cancer Lett. 194, 1-11.

Seiki, M., Koshikawa, N., Yana, I., 2003. Role of pericellular proteolysis by membrane-type 1 matrix metalloproteinase in cancer invasion and angiogenesis. Cancer Metastasis Rev. 22, 129-143.

Seizer, P., Schönberger, T., Schött, M., Lang, M.R., Langer, H.F., Bigalke, B., Krämer, B.F., Borst, O., Daub, K., Heidenreich, O., Schmidt, R., Lindemann, S., Herouy, Y., Gawaz, M., May, A.E., 2010. EMMPRIN and its ligand cyclophilin A regulate MT1-MMP, MMP-9 and M-CSF during foam cell formation. Atherosclerosis 209, 51-57.

Sekine-Aizawa, Y., Hama, E., Watanabe, K., Tsubuki, S., Kanai-Azuma, M., Kanai, Y., Arai, H., Aizawa, H., Iwata, N., Saido, T.C., 2001. Matrix metalloproteinase (MMP) system in brain: identification and characterization of brain-specific MMP highly expressed in cerebellum. Eur. J. Neurosci. 13, 935-948.

Sekiya, I., Vuoristo, J.T., Larson, B.L., Prockop, D.J., 2002. In vitro cartilage formation by human adult stem cells from bone marrow stroma defines the sequence of cellular and molecular events during chondrogenesis. Proc. Natl. Acad. Sci. USA 99, 4397-4402.

Selman, M., Ruiz, V., Cabrera, S., Segura, L., Ramirez, R., Barrios, R., Pardo, A., 2000. TIMP-1, -2, -3, and -4 in idiopathic pulmonary fibrosis. A prevailing nondegradative lung microenvironment? Am. J. Physiol. Lung Cell. Mol. Physiol. 279, L562-L574.

Sepper, R., Konttinen, Y.T., Ding, Y., Takagi, M., Sorsa, T., 1995. Human neutrophil collagenase (MMP-8), identified in bronchiectasis BAL fluid, correlates with severity of disease. Chest 107, 1641-1647.

September, A.V., Schwellnus, M.P., Collins, M., 2007. Tendon and ligament injuries: the genetic component. Br. J. Sports Med. 41, 241-246.

Sha, Q., Redman, C.M., Lee, S., 2006. Endothelin-3-converting enzyme activity of the KEL1 and KEL6 phenotypes of the Kell blood group system. J. Biol. Chem. $281,7180-7182$

Shapiro, S.D., Griffin, G.L., Gilbert, D.J., Jenkins, N.A., Copeland, N.G., Welgus, H.G., Senior, R.M., Ley, T.J., 1992. Molecular cloning, chromosomal localization, and bacterial expression of a murine macrophage metalloelastase. J. Biol. Chem. 267, 4664-4671.

Shapiro, S.D., Fliszar, C.J., Broekelmann, T.J., Mecham, R.P., Senior, R.M., Welgus, H.G., 1995. Activation of the 92-kDa gelatinase by stromelysin and 4aminophenylmercuric acetate. Differential processing and stabilization of the carboxyl-terminal domain by tissue inhibitor of metalloproteinases (TIMP). J. Biol. Chem. 270, 6351-6356.

Shapiro, S.D., 1998. Matrix metalloproteinase degradation of extracellular matrix: biological consequences. Curr. Opin. Cell Biol. 10, 602-608.

Shapiro, S.D., 2000. Evolving concepts in the pathogenesis of chronic obstructive pulmonary disease. Clin. Chest Med. 21, 621-632.

Shashkin, P., Dragulev, B., Ley, K., 2005. Macrophage differentiation to foam cells. Curr. Pharm. Des. 11, 3061-3072.

Shenkman, B., 2006. The role of ADAMTS-13 in platelet adhesion in flow: methods for diagnosis of thrombotic thrombocytopenic purpura. Pathophysiol. Haemost. Thromb. 35, 98-102.

Shimada, T., Nakamura, H., Yamashita, K., Kawata, R., Murakami, Y., Fujimoto, N., Sato, H., Seiki, M., Okada, Y., 2000. Enhanced production and activation of progelatinase A mediated by membrane-type 1 matrix metalloproteinase in human oral squamous cell carcinomas: implications for lymph node metastasis. Clin. Exp. Metastasis 18, 179-188.

Shinto, L., Marracci, G., Bumgarner, L., Yadav, V., 2011. The effects of omega-3 fatty acids on matrix metalloproteinase-9 production and cell migration in human immune cells: implications for multiple sclerosis. Autoimmune Dis. 2011, 6 [Art ID. 134592].

Shiomi, T., Lemaître, V., D’Armiento, J., Okada, Y., 2010. Matrix metalloproteinases, a disintegrin and metalloproteinases, and a disintegrin and metalloproteinases with thrombospondin motifs in non-neoplastic diseases. Pathol. Int. 60, 477-496.

Shirotani, K., Tsubuki, S., Iwata, N., Takaki, Y., Harigaya, W., Maruyama, K., Kiryu-Seo, S., Kiyama, H., Iwata, H., Tomita, T., Iwatsubo, T., Saido, T.C., 2001. Neprilysin degrades both amyloid beta peptides 1-40 and 1-42 most rapidly and efficiently among thiorphan- and phosphoramidon-sensitive endopeptidases. J. Biol. Chem. 276, 21895-21901.

Shiryaev, S.A., Savinov, A.Y., Cieplak, P., Ratnikov, B.I., Motamedchaboki, K., Smith, J.W., Strongin, A.Y., 2009a. Matrix metalloproteinase proteolysis in the myelin basic protein isoforms is a source of immunogenic peptides in autoimmune multiple sclerosis. PLoS One 4, e4952.

Shiryaev, S.A., Remacle, A.G., Savinov, A.Y., Chernov, A.V., Cieplak, P., Radichev, I.A., Williams, R., Shiryaeva, T.N., Gawlik, K., Postnova, T.I., Ratnikov, B.I., Eroshkin, A.M., Motamedchaboki, K., Smith, J.W., Strongin, A.Y., 2009b. Inflammatory proprotein convertase-matrix metalloproteinase proteolytic pathway in antigen-presenting cells as a step to autoimmune multiple sclerosis. J. Biol. Chem. 284, 30615-30626.

Shlopov, B.V., Lie, W.R., Mainardi, C.L., Cole, A.A., Chubinskaya, S., Hasty, K.A., 1997. Osteoarthritic lesions: involvement of three different collagenases. Arthritis Rheum. 40, 2065-2074.

Shofuda, K., Yasumitsu, H., Nishihashi, A., Miki, K., Miyazaki, K., 1997. Expression of three membrane-type matrix metalloproteinases (MT-MMPs) in rat vascular smooth muscle cells and characterization of MT3-MMPs with and without transmembrane domain. J. Biol. Chem. 272, 9749-9754.

Shojaei, F., Wu, X., Malik, A.K., Zhong, C., Baldwin, M.E., Schanz, S., Fuh, G., Gerber, H.P., Ferrara, N., 2007. Tumor refractoriness to anti-VEGF treatment is mediated by CD11b+Gr1+ myeloid cells. Nat. Biotechnol. 25, 911-920.

Shrimpton, C.N., Smith, A.I., Lew, R.A., 2002. Soluble metalloendopeptidases and neuroendocrine signaling. Endocr. Rev. 23, 647-664. 
Sigala, F., Savvari, P., Liontos, M., Sigalas, P., Pateras, I.S., Papalampros, A., Basdra, E.K., Kolettas, E., Kotsinas, A., Papavassiliou, A.G., Gorgoulis, V.G., 2010. Increased expression of bFGF is associated with carotid atherosclerotic plaques instability engaging the NF-kappaB pathway. J. Cell. Mol. Med. 14, 22732280.

Silence, J., Lupu, F., Collen, D., Lijnen, H.R., 2001. Persistence of atherosclerotic plaque but reduced aneurysm formation in mice with stromelysin-1 (MMP-3) gene inactivation. Arterioscler. Thromb. Vasc. Biol. 21, 1440-1445.

Siller-Lopez, F., Sandoval, A., Salgado, S., Salazar, A., Bueno, M., Garcia, J., Vera, J., Galvez, J., Hernandez, I., Ramos, M., Aguilar-Cordova, E., ArmendarizBorunda, J., 2004. Treatment with human metalloproteinase-8 gene delivery ameliorates experimental rat liver cirrhosis. Gastroenterology 126 , $1122-$ 1133.

Sims, J.D., McCready, J., Jay, D.G., 2011. Extracellular heat shock protein (Hsp)70 and Hsp90 $\alpha$ assist in matrix metalloproteinase-2 activation and breast cancer cell migration and invasion. PLoS One 6, e18848.

Sirum, K.L., Brinckerhoff, C.E., 1989. Cloning of the genes for human stromelysin and stromelysin 2: differential expression in rheumatoid synovial fibroblasts. Biochemistry 28, 8691-8698.

Skiles, J.W., Gonnella, N.C., Jeng, A.Y., 2001. The design, structure, and therapeutic application of matrix metalloproteinase inhibitors. Curr. Med. Chem. 8, 425-474.

Skiles, J.W., Gonnella, N.C., Jeng, A.Y., 2004. The design, structure, and clinical update of small molecular weight matrix metalloproteinase inhibitors. Curr. Med. Chem. 11, 2911-2977.

Skoog, T., Elomaa, O., Pasonen-Seppänen, S.M., Forsberg, S., Ahokas, K., Jeskanen, L., Pärssinen, J., Tiala, I., Rollman, O., Lohi, J., Saarialho-Kere, U., 2009. Matrix metalloproteinase-21 expression is associated with keratinocyte differentiation and upregulated by retinoic acid in HaCaT cells. J. Invest. Dermatol. 129 , 119-130.

Smith Jr., G.N., Brandt, K.D., Hasty, K.A., 1994. Procollagenase is reduced to inactive fragments upon activation in the presence of doxycycline. Ann. NY Acad. Sci. 732, 436-438.

Smith, S.M., Thomas, C.E., Birk, D.E., in press. Pericellular proteins of the developing mouse tendon: a proteomic analysis. Connect. Tissue Res.

Sohail, A., Sun, Q., Zhao, H., Bernardo, M.M., Cho, J.A., Fridman, R., 2008. MT4-(MMP17) and MT6-MMP (MMP25), a unique set of membrane-anchored matrix metalloproteinases: properties and expression in cancer. Cancer Metastasis Rev. 27, 289-302.

Sohail, A., Marco, M., Zhao, H., Shi, Q., Merriman, S., Mobashery, S., Fridman, R., 2011. Characterization of the dimerization interface of membrane type 4 (MT4)-matrix metalloproteinase (MMP). J. Biol. Chem. 286, 33178-66189.

Song, R.H., Tortorella, M.D., Malfait, A.M., Alston, J.T., Yang, Z., Arner, E.C., Griggs, D.W., 2007. Aggrecan degradation in human articular cartilage explants is mediated by both ADAMTS-4 and ADAMTS-5. Arthritis Rheum. 56, 575-585.

Song, X., Wang, X., Zhuo, W., Shi, H., Feng, D., Sun, Y., Liang, Y., Fu, Y., Zhou, D., Luo, Y., 2010. The regulatory mechanism of extracellular Hsp90\{alpha\} on matrix metalloproteinase-2 processing and tumor angiogenesis. J. Biol. Chem. 285, 40039-40049.

Sounni, N.E., Devy, L., Hajitou, A., Frankenne, F., Munaut, C., Gilles, C., Deroanne, C., Thompson, E.W., Foidart, J.M., Noel, A., 2002a. MT1-MMP expression promotes tumor growth and angiogenesis through an upregulation of vascular endothelial growth factor expression. FASEB J. 16, 555-564.

Sounni, N.E., Baramova, E.N., Munaut, C., Maquoi, E., Frankenne, F., Foidart, J.M., Noel, A., 2002b. Expression of membrane type 1 matrix metalloproteinase (MT1-MMP) in A2058 melanoma cells is associated with MMP-2 activation and increased tumor growth and vascularization. Int. J. Cancer 98, 23-28.

Sounni, N.E., Janssen, M., Foidart, J.M., Noel, A., 2003. Membrane type-1 matrix metalloproteinase and TIMP-2 in tumor angiogenesis. Matrix Biol. 22, 55-61.

Sounni, N.E., Roghi, C., Chabottaux, V., Janssen, M., Munaut, C., Maquoi, E., Galvez, B.G., Gilles, C., Frankenne, F., Murphy, G., Foidart, J.M., Noel, A., 2004. Upregulation of vascular endothelial growth factor-a by active membrane-type 1 matrix metalloproteinase through activation of src-tyrosine kinases. J. Biol. Chem. 279, 13564-13574.

Sounni, N.E., Rozanov, D.V., Remacle, A.G., Golubkov, V.S., Noel, A., Strongin, A.Y., 2010. TIMP-2 binding with cellular MT1-MMP stimulates invasionpromoting MEK/ERK signaling in cancer cells. Int. J. Cancer 126, 1067-1078.

Spinale, F.G., 2007. Myocardial matrix remodeling and the matrix metalloproteinases: influence on cardiac form and function. Physiol. Rev. 87, 1285-1342.

Sreedharan, S.P., Goetzl, E.J., Malfroy, B., 1990. Elevated synovial tissue concentration of the common acute lymphoblastic leukaemia antigen (CALLA)associated neutral endopeptidase (3.4.24.11) in human chronic arthritis. Immunology 71, 142-144.

Stack, M.S., Gray, R.D., 1989. Comparison of vertebrate collagenase and gelatinase using a new fluorogenic substrate peptide. J. Biol. Chem. 264, 4277-4281.

Stack, M.S., Gray, R.D., 1990. The effect of pH, temperature, and D2O on the activity of porcine synovial collagenase and gelatinase. Arch. Biochem. Biophys. 281, 257-263.

Stadlmann, S., Pollheimer, J., Moser, P.L., Raggi, A., Amberger, A., Margreiter, R., Offner, F.A., Mikuz, G., Dirnhofer, S., Moch, H., 2003. Cytokine-regulated expression of collagenase-2 (MMP-8) is involved in the progression of ovarian cancer. Eur. J. Cancer 39, $2499-2505$.

Ståhle-Bäckdahl, M., Sandstedt, B., Bruce, K., Lindahl, A., Jimenez, M.G., Vega, J.A., Lopez-Otin, C., 1997. Collagenase-3 (MMP-13) is expressed during human fetal ossification and re-expressed in postnatal bone remodeling and in rheumatoid arthritis. Lab. Invest. 76, 717-728.

Standeven, K.F., Hess, K., Carter, A.M., Rice, G.I., Cordell, P.A., Balmforth, A.J., Lu, B., Scott, D.J., Turner, A.J., Hooper, N.M., Grant, P.J., 2011. Neprilysin, obesity and the metabolic syndrome. Int. J. Obes. 35, 1031-1040.

Stankovic, S., Konjevic, G., Gopcevic, K., Jovic, V., Inic, M., Jurisic, V., 2010. Activity of MMP-2 and MMP-9 in sera of breast cancer patients. Pathol. Res. Pract. 206, 241-247.

Stanton, H., Rogerson, F.M., East, C.J., Golub, S.B., Lawlor, K.E., Meeker, C.T., Little, C.B., Last, K., Farmer, P.J., Campbell, I.K., Fourie, A.M., Fosang, A.J., 2005. ADAMTS5 is the major aggrecanase in mouse cartilage in vivo and in vitro. Nature 434, 648-652.

Steenport, M., Khan, K.M., Du, B., Barnhard, S.E., Dannenberg, A.J., Falcone, D.J., 2009. Matrix metalloproteinase (MMP)-1 and MMP-3 induce macrophage MMP-9: evidence for the role of TNF-alpha and cyclooxygenase-2. J. Immunol. 183, 8119-8127.

Stefanidakis, M., Koivunen, E., 2006. Cell-surface association between matrix metalloproteinases and integrins: role of the complexes in leukocyte migration and cancer progression. Blood 108, 1441-1450.

Steffensen, B., Wallon, U.M., Overall, C.M., 1995. Extracellular matrix binding properties of recombinant fibronectin type II-like modules of human 72-kDa gelatinase/type IV collagenase. High affinity binding to native type I collagen but not native type IV collagen. J. Biol. Chem. $270,11555-11566$.

Stenman, M., Paju, A., Hanemaaijer, R., Tervahartiala, T., Leminen, A., Stenman, U.H., Konttinen, Y.T., Sorsa, T., 2003. Collagenases (MMP-1-8 and -13) and trypsinogen-2 in fluid from benign and malignant ovarian cysts. Tumour Biol. 24, 9-12.

Sterchi, E.E., Stöcker, W., Bond, J.S., 2008. Meprins, membrane-bound and secreted astacin metalloproteinases. Mol. Aspects Med. 29, $309-328$.

Sternberg, Z., Chadha, K., Lieberman, A., Hojnacki, D., Drake, A., Zamboni, P., Rocco, P., Grazioli, E., Weinstock-Guttman, B., Munschauer, F., 2008. Quercetin and interferon-beta modulate immune response(s) in peripheral blood mononuclear cells isolated from multiple sclerosis patients. J. Neuroimmunol. 205, 142-147.

Sternlicht, M., Lochter, A., Sympson, C.J., Huey, B., Rougier, J.P., Gray, J.W., Pinkel, D., Bissell, M.J., Werb, Z., 1999. The stromal proteinase MMP3/stromelysin-1 promotes mammary carcinogenesis. Cell 98, 137-146.

Sternlicht, M.D., Bissell, M.J., Werb, Z., 2000. The matrix metalloproteinase stromelysin-1 acts as a natural mammary tumor promoter. Oncogene 19, 1910219113.

Sternlicht, M.D., Werb, Z., 2001. How matrix metalloproteinases regulate cell behavior. Annu. Rev. Cell Dev. Biol. 17, 463-516.

Stetler-Stevenson, W.G., 1999. Matrix metalloproteinases in angiogenesis: a moving target for therapeutic intervention. J. Clin. Invest. 103, 1237-1241.

Stetler-Stevenson, W.G., Yu, A.E., 2001. Proteases in invasion: matrix metalloproteinases. Semin. Cancer Biol. 11, 143-152.

Stetler-Stevenson, W.G., 2008. Tissue inhibitors of metalloproteinases in cell signaling: metalloproteinase-independent activities. Sci. Signal 1, re6.

Stocker, W., Bode, W., 1995. Structural features of a superfamily of zinc-endopeptidases: the metzincins. Curr. Opin. Struct. Biol. 5, $383-390$.

Stojic, J., Hagemann, C., Haas, S., Herbold, C., Kühnel, S., Gerngras, S., Roggendorf, W., Roosen, K., Vince, G.H., 2008. Expression of matrix metalloproteinases MMP-1, MMP-11 and MMP-19 is correlated with the WHO-grading of human malignant gliomas. Neurosci. Res. 60, 40-49. 
Stone, P.J., Gottlieb, D.J., O’Connor, G.T., Ciccolella, D.E., Breuer, R., Bryan-Rhadfi, J., Shaw, H.A., Franzblau, C., Snider, G.L., 1995. Elastin and collagen degradation products in urine of smokers with and without chronic obstructive pulmonary disease. Am. J. Respir. Crit. Care Med. 151, 952-959.

Stracke, J.O., Hutton, M., Stewart, M., Pendas, A.M., Smith, B., Lopez-Otin, C., Murphy, G., Knäuper, V., 2000. Biochemical characterization of the catalytic domain of human matrix metalloproteinase 19. Evidence for a role as a potent basement membrane degrading enzyme. J. Biol. Chem. 275, 1480914816.

Su, L., Zhou, W., Asomaning, K., Lin, X., Wain, J.C., Lynch, T.J., Liu, G., Christiani, D.C., 2006. Genotypes and haplotypes of matrix metalloproteinase 1, 3 and 12 genes and the risk of lung cancer. Carcinogenesis 27, 1024-1029.

Sumitomo, M., Iwase, A., Zheng, R., Navarro, D., Kaminetzky, D., Shen, R., Georgescu, M.M., Nanus, D.M., 2004. Synergy in tumor suppression by direct interaction of neutral endopeptidase with PTEN. Cancer Cell 5, 67-78.

Sumitomo, M., Shen, R., Nanus, D.M., 2005. Involvement of neutral endopeptidase in neoplastic progression. Biochim. Biophys. Acta $1751,52-59$.

Sun, Y.N., Li, Y., 2004. Expression of mRNA for membrane-type 1, 2, and 3 matrix metalloproteinases in human laryngeal cancer. Chin. Med. Sci. J. 19, 170173.

Sun, T., Gao, Y., Tan, W., Ma, S., Zhang, X., Wang, Y., Zhang, Q., Guo, Y., Zhao, D., Zeng, C., Lin, D., 2006. Haplotypes in matrix metalloproteinase gene cluster on chromosome 11q22 contribute to the risk of lung cancer development and progression. Clin. Cancer Res. 12, $7009-7017$.

Sun, Q., Weber, C.R., Sohail, A., Bernardo, M.M., Toth, M., Zhao, H., Turner, J.R., Fridman, R., 2007. MMP-25 (MT6-MMP) is highly expressed in human colon cancer, promotes tumor growth, and exhibits unique biochemical properties. J. Biol. Chem. 282, 21998-22010.

Sun, L., Diamond, M.E., Ottaviano, A.J., Joseph, M.J., Ananthanarayan, V., Munshi, H.G., 2008. Transforming growth factor-beta 1 promotes matrix metalloproteinase-9-mediated oral cancer invasion through snail expression. Mol. Cancer Res. 6, 10-20.

Suomela, S., Koljonen, V., Skoog, T., Kukko, H., Böhling, T., Saarialho-Kere, U., 2009. Expression of MMP-10, MMP-21, MMP-26, and MMP-28 in Merkel cell carcinoma. Virchows Arch. 455, 495-503.

Swaisgood, C.M., French, E.L., Noga, C., Simon, R.H., Ploplis, V.A., 2000. The development of bleomycin-induced pulmonary fibrosis in mice deficient for components of the fibrinolytic system. Am. J. Pathol. 157, 177-187.

Swartz, M.A., Tschumperlin, D.J., Kamm, R.D., Drazen, J.M., 2001. Mechanical stress is communicated between different cell types to elicit matrix remodeling. Proc. Natl. Acad. Sci. USA 98, 6180-6185.

Swiderski, R.E., Dencoff, J.E., Floerchinger, C.S., Shapiro, S.D., Hunninghake, G.W., 1998. Differential expression of extracellular matrix remodelling genes in a murine model of bleomycin-induced pulmonary fibrosis. Am. J. Pathol. 152, 821-828.

Szabova, L., Yamada, S.S., Wimer, H., Chrysovergis, K., Ingvarsen, S., Behrendt, N., Engelholm, L.H., Holmbeck, K., 2009. MT1-MMP and type II collagen specify skeletal stem cells and their bone and cartilage progeny. J. Bone Miner. Res. 24, 1905-1916.

Szabova, L., Son, M.Y., Shi, J., Sramko, M., Yamada, S.S., Swaim, W.D., Zerfas, P., Kahan, S., Holmbeck, K., 2010. Membrane-type MMPs are indispensable for placental labyrinth formation and development. Blood 116, 5752-5761.

Taddese, S., Jung, M.C., Ihling, C., Heinz, A., Neubert, R.H.H., Schmelzer, C.E.H., 2010. MMP-12 catalytic domain recognizes and cleaves at multiple sites in human skin collagen type I and type III. Biochim. Biophys. Acta 1804, 731-739.

Tak, P.P., Smeets, T.J., Daha, M.R., Kluin, P.M., Meijers, K.A., Brand, R., Meinders, A.E., Breedveld, F.C., 1997. Analysis of the synovial cell infiltrate in early rheumatoid synovial tissue in relation to local disease activity. Arthritis Rheum. 40, 217-225.

Takaishi, H., Kimura, T., Dalal, S., Okada, Y., D’Armiento, J., 2008. Joint diseases and matrix metalloproteinases: a role for MMP-13. Curr. Pharm. Biotechnol. 9, 47-54.

Takino, T., Sato, H., Shinagawa, A., Seiki, M., 1995. Identification of the second membrane-type matrix metalloproteinase (MT-MMP-2) gene from a human placenta cDNA library. MT-MMPs form a unique membrane-type subclass in the MMP family. J. Biol. Chem. 270, 23013-23020.

Takino, T., Koshikawa, N., Miyamori, H., Tanaka, M., Sasaki, T., Okada, Y., Seiki, M., Sato, H., 2003. Cleavage of metastasis suppressor gene product KiSS-1 protein/metastin by matrix metalloproteinases. Oncogene 22, 4617-4626.

Tam, E.R., Wu, Y.I., Butler, G.S., Stack, M.S., Overall, C.M., 2002. Collagen binding properties of the membrane type-1 matrix metalloproteinase (MT1-MMP) hemopexin C domain. The ectodomain of the 44-kDa autocatalytic product of MT1-MMP inhibits cell invasion by disrupting native type I collagen cleavage. J. Biol. Chem. 277, 39005-39014.

Tam, E.R., Moore, T.D., Butler, G.S., Overall, C.M., 2004. Characterization of the distinct collagen binding, helicase and cleavage mechanisms of matrix metalloproteinase 2 and 14 (gelatinase A and MT1-MMP): the differential roles of the MMP hemopexin c domains and the MMP-2 fibronectin type II modules in collagen triple helicase activities. J. Biol. Chem. 279, 43336-43344.

Tamamura, Y., Otani, T., Kanatani, N., Koyama, E., Kitagaki, J., Komori, T., Yamada, Y., Costantini, F., Wakisaka, S., Pacifici, M., Iwamoto, M., Enomoto-Iwamoto, M., 2005. Developmental regulation of Wnt/beta-catenin signals is required for growth plate assembly, cartilage integrity, and endochondral ossification. J. Biol. Chem. 280, 19185-19195.

Tan, X., Egami, H., Ishikawa, S., Sugita, H., Kamohara, H., Nakagawa, M., Nozawa, F., Abe, M., Ogawa, M., 2005. Involvement of matrix metalloproteinase-7 in invasion-metastasis through induction of cell dissociation in pancreatic cancer. Int. J. Oncol 26, 1283-1289.

Tanaka, M., Itai, T., Adachi, M., Nagata, S., 1998. Downregulation of Fas ligand by shedding. Nat. Med. 4, 31-36.

Tang, N., Xie, Q., Wang, X., Li, X., Chen, Y., Lin, X., Lin, J., 2011. Inhibition of invasion and metastasis of MHCC97H cells by expression of snake venom cystatin through reduction of proteinases activity and Epithelial-Mesenchymal Transition. Arch. Pharm. Res. 34, 781-789.

Tanner, R.M., Lynch, A.I., Brophy, V.H., Eckfeldt, J.H., Davis, B.R., Ford, C.E., Boerwinkle, E., Arnett, D.K., 2011. Pharmacogenetic associations of MMP9 and MMP12 variants with cardiovascular disease in patients with hypertension. PLoS One 6, e23609.

Tchetverikov, I., Lard, L.R., DeGroot, J., Verzijl, N., TeKoppele, J.M., Breedveld, F.C., Huizinga, T.W., Hanemaaijer, R., 2003. Matrix metalloproteinases-3, -8, -9 as markers of disease activity and joint damage progression in early rheumatoid arthritis. Ann. Rheum. Dis. 62, 1094-1099.

Tenenhouse, H.S., 1999. X-linked hypophosphataemia: a homologous disorder in humans and mice. Nephrol. Dial. Transplant. 14, 333-341.

Tester, A.M., Waltham, M., Oh, S.J., Bae, S.N., Bills, M.M., Walker, E.C., Kern, F.G., Stetler-Stevenson, W.G., Lippman, M.E., Thompson, E.W., 2004. Pro-matrix metalloproteinase-2 transfection increases orthotopic primary growth and experimental metastasis of MDA-MB-231 human breast cancer cells in nude mice. Cancer Res. 64, 652-658.

Tester, A.M., Cox, J.H., Connor, A.R., Starr, A.E., Dean, R.A., Puente, X.S., Lopez-Otin, C., Overall, C.M., 2007. LPS responsiveness and neutrophil chemotaxis in vivo require PMN MMP-8 activity. PLoS One 2, e312.

Tetlow, L.C., Woolley, D.E., 1998. Comparative immunolocalization studies of collagenase 1 and collagenase 3 production in the rheumatoid lesion, and by human chondrocytes and synoviocytes in vitro. Br. J. Rheumatol. 37, 64-70.

Tetlow, L.C., Adlam, D.J., Woolley, D.E., 2001. Matrix metalloproteinase and proinflammatory cytokine production by chondrocytes of human osteoarthritic cartilage: associations with degenerative changes. Arthritis Rheum. 44, 585-594.

Théret, N., Musso, O., L'Helgoualc'h, A., Campion, J.P., Clement, B., 1998. Differential expression and origin of membrane-type 1 and 2 matrix metalloproteinases (MT-MMPs) in association with MMP2 activation in injured human livers. Am. J. Pathol. 153, 945-954.

Thewes, M., Worret, W.I., Engst, R., Ring, J., 1999. Stromelysin-3 (ST-3): immunohistochemical characterization of the matrix metalloproteinase (MMP)-11 in benign and malignant skin tumours and other skin disorders. Clin. Exp. Perinatol. 24, 122-126.

Thodeti, C.K., Albrechtsen, R., Grauslund, M., Asmar, M., Larsson, C., Takada, Y., Mercurio, A.M., Couchman, J.R., Wewer, U.M., 2003. ADAM12/syndecan-4 signaling promotes beta 1 integrin-dependent cell spreading through protein kinase Calpha and RhoA. J. Biol. Chem. $278,9576-9584$.

Thomasset, N., Lochter, A., Sympson, C.J., Lund, L.R., Williams, D.R., Behrendtsen, O., Werb, Z., Bissell, M.J., 1998. Expression of autoactivated stromelysin-1 in mammary glands of transgenic mice leads to a reactive stroma during early development. Am. J. Pathol. $153,457-467$.

Thompson, R.W., Geraghty, P.J., Lee, J.K., 2002. Abdominal aortic aneurysms: basic mechanisms and clinical implications. Curr. Probl. Surg. 39, 110-230.

Titz, B., Dietrich, S., Sadowski, T., Beck, C., Petersen, A., Sedlacek, R., 2004. Activity of MMP-19 inhibits capillary-like formation due to processing of nidogen1. Cell. Mol. Life Sci. 61, 1826-1833. 
Todorova, L., Bjermer, L., Miller-Larsson, A., Westergren-Thorsson, G., 2010. Relationship between matrix production by bronchial fibroblasts and lung function and AHR in asthma. Respir. Med. 104, 1799-1808.

Toft-Hansen, H., Nuttall, R.K., Edwards, D.R., Owens, T., 2004. Key metalloproteinases are expressed by specific cell types in experimental autoimmune encephalomyelitis. J. Immunol. 173, 5209-5218.

Tortorella, M.D., Malfait, F., Barve, R.A., Shieh, H.S., Malfait, A.M., 2009. A review of the ADAMTS family, pharmaceutical targets of the future. Curr. Pharm. 15, $2359-2374$.

Tousseyn, T., Jorissen, E., Reiss, K., Hartmann, D., 2006. (Make) stick and cut loose-disintegrin metalloproteases in development and disease. Birth Defects Res. C Embryo Today 78, 24-46.

Tran-Paterson, R., Willard, H.F., Letarte, M., 1989. The common acute lymphoblastic leukemia antigen (neutral endopeptidase-3.4.24.11) gene is located on human chromosome 3. Cancer Genet. Cytogenet. 42, 129-134.

Trapp, B.D., Nishiyama, A., Cheng, D., Macklin, W., 1997. Differentiation and death of premyelinating oligodendrocytes in developing rodent brain. J. Cell Biol. 137, 459-468

Trexler, M., Briknarov, K., Gehrmann, M., Llinas, M., Patthy, L., 2003. Peptide ligands for the fibronectin type II modules of matrix metalloproteinase 2 (MMP2). J. Biol. Chem. 278, 12241-12246.

Tromp, G., Gatalica, Z., Skunca, M., Berguer, R., Siegel, T., Kline, R.A., Kuivaniemi, H., 2004. Elevated expression of matrix metalloproteinase-13 in abdominal aortic aneurysms. Ann. Vasc. Surg. 18, 414-420.

Tsukada, H., Pourmotabbed, T., 2002. Unexpected crucial role of residue 272 in substrate specificity of fibroblast collagenase. J. Biol. Chem. 277, 2737827384.

Tsunezuka, Y., Kinoh, H., Takino, T., Watanabe, Y., Okada, Y., Shinagawa, A., Sato, H., Seiki, M., 1996. Expression of membrane-type matrix metalloproteinase 1 (MT1-MMP) in tumor cells enhances pulmonary metastasis in an experimental metastasis assay. Cancer Res. 56, $5678-5683$.

Tu, X.K., Yang, W.Z., Liang, R.S., Shi, S.S., Chen, J.P., Chen, C.M., Wang, C.H., Xie, H.S., Chen, Y., Ouyang, L.Q., 2011. Effect of baicalin on matrix metalloproteinase-9 expression and blood-brain barrier permeability following focal cerebral ischemia in rats. Neurochem. Res. 36, $2022-2028$.

Turner, A.J., Isaac, R.E., Coates, D., 2001. The neprilysin (NEP) family of zinc metalloendopeptidases: genomics and function. Bioessays 23, 261-269.

Turner, A.J., 2003. Exploring the structure and function of zinc metallopeptidases: old enzymes and new discoveries. Biochem. Soc. Trans. 31, $723-727$.

Turner, A.J., Nalivaeva, N.N., 2006. Proteinase dysbalance in pathology: the neprilysin (NEP) and angiotensin-converting enzyme (ACE) families. Cell. Mol. Biol. 52, 40-48.

Turpeenniemi-Hujanen, T., 2005. Gelatinases (MMP-2 and -9) and their natural inhibitors as prognostic indicators in solid cancers. Biochimie 87, 287-297.

Turu, M.M., Krupinski, J., Catena, E., Rosell, A., Montaner, J., Rubio, F., Alvarez-Sabin, J., Cairols, M., Badimon, L., 2006. Intraplaque MMP-8 levels are increased in asymptomatic patients with carotid plaque progression on ultrasound. Atherosclerosis 187, 161-169.

Uchinami, H., Seki, E., Brenner, D.A., D’Armiento, J., 2006. Loss of MMP-13 attenuates murine hepatic injury and fibrosis during cholestasis. Hepatology 44 420-429.

Udenfriend, S., Kodukula, K., 1995. How glycosylphosphatidylinositol-anchored membrane proteins are made. Annu. Rev. Biochem. 64, 563-591.

Ueda, J., Kajita, M., Suenaga, N., Fujii, K., Seiki, M., 2003. Sequence-specific silencing of MT1-MMP expression suppresses tumor cell migration and invasion: importance of MT1-MMP as a therapeutic target for invasive tumors. Oncogene 22, 8716-8722.

Uekita, T., Gotoh, I., Kinoshita, T., Itoh, Y., Sato, H., Shiomi, T., Okada, Y., Seiki, M., 2004. Membrane-type 1 matrix metalloproteinase cytoplasmic tail-binding protein-1 is a new member of the cupin superfamily: a possible multifunctional protein acting as an invasion suppressor down-regulated in tumors. J. Biol. Chem. 279, 12734-12743.

Ueno, H., Nakamura, H., Inoue, M., Imai, K., Noguchi, M., Sato, H., Seiki, M., Okada, Y., 1997. Expression and tissue localization of membranetypes 1, 2, and 3 matrix metalloproteinases in human invasive breast carcinomas. Cancer Res. 57, 2055-2060.

Uitto, V.J., Airola, K., Vaalamo, M., Johansson, N., Putnins, E.E., Firth, J.D., Salonen, J., Lopez-Otin, C., Saarialho-Kere, U., Kähäri, V.M., 1998. Collagenase-3 (matrix metalloproteinase-13) expression is induced in oral mucosal epithelium during chronic inflammation. Am. J. Pathol. 152, 1489-1499.

Ulrich, R., Gerhauser, I., Seeliger, F., Baumgärtner, W., Alldinger, S., 2005. Matrix metalloproteinases and their inhibitors in the developing mouse brain and spinal cord: a reverse transcription quantitative polymerase chain reaction study. Dev. Neurosci. 27, 408-418.

Uria, J.A., Balbin, M., Lopez, J.M., Alvarez, J., Vizoso, F., Takigawa, M., Lopez-Otin, C., 1998. Collagenase-3 (MMP-13) expression in chondrosarcoma cells and its regulation by basic fibroblast growth factor. Am. J. Pathol. 153, 91-101.

Uria, J.A., Lopez-Otin, C., 2000. Matrilysin-2, a new matrix metalloproteinase expressed in human tumors and showing the minimal domain organization required for secretion, latency, and activity. Cancer Res. 60, 4745-4751.

Usmani, B.A., Shen, R., Janeczko, M., Papandreou, C.N., Lee, W.H., Nelson, W.G., Nelson, J.B., Nanus, D.M., 2000. Methylation of the neutral endopeptidase gene promoter in human prostate cancers. Clin. Cancer Res. 6, 1664-1670.

Vaalamo, M., Mattila, L., Johansson, N., Kariniemi, A.L., Karjalainen-Lindsberg, M.L., Kähäri, V.M., Saarialho-Kere, U., 1997. Distinct populations of stromal cells express collagenase-3 (MMP-13) and collagenase-1 (MMP-1) in chronic ulcers, but not in normally healing wounds. J. Invest. Dermatol. 109, 96101.

Vaalamo, M., Karjalainen-Lindsberg, M.L., Puolakkainen, P., Kere, J., Saarialho-Kere, U., 1998. Distinct expression profiles of stromelysin-2 (MMP-10), collagenase-3 (MMP-13), macrophage metalloelastase (MMP-12), and tissue inhibitor of metalloproteinases-3 (TIMP-3) in intestinal ulcerations. Am. J. Pathol. 152, 1005-1014.

Vaillant, C., Didier-Baze‘s, M., Hutter, A., Belin, M.F., Thomasset, N., 1999. Spatiotemporal expression patterns of metalloproteinases and their inhibitors in the postnatal developing rat cerebellum. J. Neurosci. 19, 4994-5004.

Valença, S.S., Da Hora, K., Castro, P., Gonçalves de Moraes, V., Carvalho, L., Moraes Sobrino Porto, L.C., 2004. Emphysema and metalloelastase expression in mouse lung induced by cigarette smoke. Toxicol. Pathol. 32, 351-356.

Valkovskaya, N., Kayed, H., Felix, K., Hartmann, D., Giese, N.A., Osinsky, S.P., Friess, H., Kleeff, J., 2007. ADAM8 expression is associated with increased invasiveness and reduced patient survival in pancreatic cancer. J. Cell. Mol. Med. 11, 1162-1174.

Van den Steen, P., Rudd, P.M., Dwek, R.A., Van Damme, J., Opdenakker, G., 1998. Cytokine and protease glycosylation as a regulatory mechanism in inflammation and autoimmunity. Adv. Exp. Med. Biol. 435, 133-143.

Van den Steen, P.E., Proost, P., Wuyts, A., van Damme, J., Opdenakker, G., 2000. Neutrophil gelatinase B potentiates interleukin-8 tenfold by aminoterminal processing, whereas it degrades CTAP-III, PF-4, and GRO-alpha and leaves RANTES and MCP-2 intact. Blood 96, 2673-2681.

Van den Steen, P.E., Dubois, B., Nelissen, I., Rudd, P.M., Dwek, R.A., Opdenakker, G., 2002. Biochemistry and molecular biology of gelatinase B or matrix metalloproteinase-9 (MMP-9). Crit. Rev. Biochem. Mol. Biol. 37, 375-536.

Van den Steen, P.E., Husson, S.J., Proost, P., Van Damme, J., Opdenakker, G., 2003a. Carboxyterminal cleavage of the chemokines MIG and IP-10 by gelatinase B and neutrophil collagenase. Biochem. Biophys. Res. Commun. 310, 889-896.

Van den Steen, P.E., Wuyts, A., Husson, S.J., Proost, P., Van Damme, J., Opdenakker, G., 2003b. Gelatinase B/MMP-9 and neutrophil collagenase/MMP-8 process the chemokines human GCP-2/CXCL6, ENA-78/CXCL5 and mouse GCP-2/LIX and modulate their physiological activities. Eur. J. Biochem. 270, 3739-3749.

Van den Steen, P.E., Proost, P., Brand, D.D., Kang, A.H., Van Damme, J., Opdenakker, G., 2004. Generation of glycosylated remnant epitopes from human collagen type II by gelatinase B. Biochemistry 43, 10809-10816.

Van den Steen, P.E., Grillet, B., Opdenakker, G., 2005. Gelatinase B participates in collagen II degradation and releases glycosylated remnant epitopes in rheumatoid arthritis. Adv. Exp. Med. Biol. 564, 45-55.

Van den Steen, P.E., Van Aelst, I., Hvidberg, V., Piccard, H., Fiten, P., Jacobsen, C., Moestrup, S.K., Fry, S., Royle, L., Wormald, M.R., Wallis, R., Rudd, P.M., Dwek, R.A., Opdenakker, G., 2006. The hemopexin and O-glycosylated domains tune gelatinase B/MMP-9 bioavailability via inhibition and binding to cargo receptors. J. Biol. Chem. 281, 18626-18637. 
Van der Vaart, H., Postma, D.S., Timens, W., ten Hacken, N.H., 2004. Acute effects of cigarette smoke on inflammation and oxidative stress: a review. Thorax 59, 713-721.

Van Eerdewegh, P., Little, R.D., Dupuis, J., Del Mastro, R.G., Falls, K., Simon, J., Torrey, D., Pandit, S., McKenny, J., Braunschweiger, K., Walsh, A., Liu, Z., Hayward, B., Folz, C., Manning, S.P., Bawa, A., Saracino, L., Thackston, M., Benchekroun, Y., Capparell, N., Wang, M., Adair, R., Feng, Y., Dubois, J., FitzGerald, , Huang, H., Gibson, R., Allen, K.M., Pedan, A., Danzig, M.R., Umland, S.P., Egan, R.W., Cuss, F.M., Rorke, S., Clough, J.B., Holloway, J.W., Holgate, S.T., Keith, T.P., 2002. Association of the ADAM33 gene with asthma and bronchial hyperresponsiveness. Nature 418, $426-430$.

Van Goor, H., Melenhorst, W.B., Turner, A.J., Holgate, S.T., 2009. Adamalysins in biology and disease. J. Pathol. 219, 277-286.

Van Heesch, P.N., Struijk, P.C., Laudy, J.A., Steegers, E.A., Wildschut, H.I., 2010. Estimating the effect of gestational age on test performance of combined firsttrimester screening for Down syndrome: a preliminary study. J. Perinat. Med. 38, 305-309.

Van Horssen, J., Vos, C.M., Admiraal, L., van Haastert, E.S., Montagne, L., van der Valk, P., de Vries, H.E., 2006. Matrix metalloproteinase-19 is highly expressed in active multiple sclerosis lesions. Neuropathol. Appl. Neurobiol. 32, 585-593.

Van Lint, P., Wielockx, B., Puimege, L., Noel, A., Lopez-Otin, C., Libert, C., 2005. Resistance of collagenase-2 (matrix metalloproteinase-8)-deficient mice to TNF-induced lethal hepatitis. J. Immunol. 175, 7642-7649.

Van Lint, P., Libert, C., 2006. Matrix metalloproteinase-8: cleavage can be decisive. Cytokine Growth Factor Rev. 17, 217-223.

Van Meurs, J., van Lent, P., Stoop, R., Holthuysen, A., Singer, I., Bayne, E., Mudgett, J., Poole, R., Billinghurst, C., van der Kraan, P., Buma, P., van den Berg, W., 1999. Cleavage of aggrecan at the $\mathrm{Asn}^{341}-\mathrm{Phe}^{342}$ site coincides with the initiation of collagen damage in murine antigen-induced arthritis: a pivotal role for stromelysin 1 in matrix metalloproteinase activity. Arthritis Rheum. 42, 2074-2084.

Van Noort, J.M., Amor, S., 1998. Cell biology of autoimmune diseases. Int. Rev. Cytol. 178, 127-206.

Van Vollenhoven, R.F., 2011. Unresolved issues in biologic therapy for rheumatoid arthritis. Nat. Rev. Rheumatol. 7, 205-215.

Vargo-Gogola, T., Crawford, H.C., Fingleton, B., Matrisian, L.M., 2002. Identification of novel matrix metalloproteinase-7 (matrilysin) cleavage sites in murine and human Fas ligand. Arch. Biochem. Biophys. 408, 155-161.

Vazquez, F., Hastings, G., Ortega, M.A., Lane, T.F., Oikemus, S., Lombardo, M., Iruela-Arispe, M.L., 1999. METH-1, a human ortholog of ADAMTS-1, and METH-2 are members of a new family of proteins with angio-inhibitory activity. J. Biol. Chem. 274, 23349-23357.

Veeravalli, K.K., Chetty, C., Ponnala, S., Gondi, C.S., Lakka, S.S., Fassett, D., Klopfenstein, J.D., Dinh, D.H., Gujrati, M., Rao, J.S., 2010. MMP-9, uPAR and cathepsin B silencing downregulate integrins in human glioma xenograft cells in vitro and in vivo in nude mice. PLoS One 5, e11583.

Velasco, G., Cal, S., Merlos-Suarez, A., Ferrando, A.A., Alvarez, S., Nakano, A., Arribas, J., Lopez-Otin, C., 2000. Human MT6-matrix metalloproteinase: identification, progelatinase A activation, and expression in brain tumors. Cancer Res. 60, 877-882.

Velasco-Loyden, G., Arribas, J., Lopez-Casillas, F., 2004. The shedding of betaglycan is regulated by pervanadate and mediated by membrane type matrix metalloprotease-1. J. Biol. Chem. 279, 7721-7733.

Velinov, N., Aebersold, D., Haeni, N., Hlushchuk, R., Mishev, G., Weinstein, F., Sedlacek, R., Djonov, V., 2007. Matrix metalloproteinase-19 is a predictive marker for tumor invasiveness in patients with oropharyngeal squamous cell carcinoma. Int. J. Biol. Markers 22, $265-273$.

Vermeer, P.D., Denker, J., Estin, M., Moninger, T.O., Keshavjee, S., Karp, P., Kline, J.N., Zabner, J., 2009. MMP9 modulates tight junction integrity and cell viability in human airway epithelia. Am. J. Physiol. Lung Cell. Mol. Physiol. 296, L751-L762.

Vernier, P., Moret, F., Callier, S., Snapyan, M., Wersinger, C., Sidhu, A., 2004. The degeneration of dopamine neurons in Parkinson's disease: insights from embryology and evolution of the mesostriatocortical system. Ann. NY Acad. Sci. 1035, 231-249.

Verraes, S., Hornebeck, W., Polette, M., Borradori, L., Bernard, P., 2001. Respective contribution of neutrophil elastase and matrix metalloproteinase 9 in the degradation of BP180 (type XVII collagen) in human bullous pemphigoid. J. Invest. Dermatol. 117, 1091-1106.

Viappiani, S., Sariahmetoglu, M., Schulz, R., 2006. The role of matrix metalloproteinase inhibitors in ischemia-reperfusion injury in the liver. Curr. Pharm. Des. 12, 2923-2934.

Vincenti, M.P., Brinckerhoff, C.E., 2002. Transcriptional regulation of collagenase (MMP-1, MMP-13) genes in arthritis: integration of complex signaling pathways for the recruitment of genespecific transcription factors. Arthritis Res. 4, 157-164.

Visse, R., Nagase, H., 2003. Matrix metalloproteinases and tissue inhibitors of metalloproteinases: structure, function, and biochemistry. Circ. Res. 92, 827839.

Volcik, K.A., Campbell, S., Chambless, L.E., Coresh, J., Folsom, A.R., Mosley, T.H., Ni, H., Wagenknecht, L.E., Wasserman, B.A., Boerwinkle, E., 2010. MMP2 genetic variation is associated with measures of fibrous cap thickness: the Atherosclerosis Risk in Communities Carotid MRI Study. Atherosclerosis 210, $188-193$.

Vos, C.M., van Haastert, E.S., de Groot, C.J., van der Valk, P., de Vries, H.E., 2003. Matrix metalloproteinase-12 is expressed in phagocytotic macrophages in active multiple sclerosis lesions. J. Neuroimmunol. 138, 106-114.

Vu, T.H., Shipley, J.M., Bergers, G., Berger, J.E., Helms, J.A., Hanahan, D., Shapiro, S.D., Senior, R.M., Werb, Z., 1998. MMP-9/gelatinase B is a key regulator of growth plate angiogenesis and apoptosis of hypertrophic chondrocytes. Cell 93, 411-422.

Vu, T.H., Werb, Z., 2000. Matrix metalloproteinases: effectors of development and normal physiology. Genes Dev. 14, 2123-2133.

Wågsäter, D., Bjork, H., Zhu, C., Bjorkegren, J., Valen, G., Hamsten, A., Eriksson, P., 2008. ADAMTS-4 and -8 are inflammatory regulated enzymes expressed in macrophage-rich areas of human atherosclerotic plaques. Atherosclerosis 196, 514-522.

Wågsäter, D., Zhu, C., Björkegren, J., Skogsberg, J., Eriksson, P., 2011. MMP-2 and MMP-9 are prominent matrix metalloproteinases during atherosclerosis development in the Ldlr(-/-)Apob(100/100) mouse. Int. J. Mol. Med. 28, 247-253.

Wagstaff, L., Kelwick, R., Decock, J., Edwards, D.R., 2011. The roles of ADAMTS metalloproteinases in tumorigenesis and metastasis. Front. Biosci. 16, 18611872 .

Wald, N.J., Watt, H.C., Hackshaw, A.K., 1999. Integrated screening for Down's syndrome based on tests performed during the first andsecond trimesters. N. Engl. J. Med. 341, 461-467.

Walker, M.E., Hatfield, J.K., Brown, M.A., in press. New insights into the role of mast cells in autoimmunity: evidence for a common mechanism of action? Biochim. Biophys. Acta.

Wallard, M.J., Pennington, C.J., Veerakumarasivamm, A., Burtt, G., Mills, I.G., Warren, A., Leung, H.Y., Murphy, G., Edwards, D.R., Neal, D.E., Kelly, J.D., 2006. Comprehensive profiling and localisation of the matrix metalloproteinases in urothelial carcinoma. Br. J. Cancer 94, 569-577.

Walling, H.W., Chan, P.T., Omuram, T.H., Barmina, O.Y., Fiacco, G.J., Jeffrey, J.J., Partridge, N.C., 1998. Regulation of the collagenase-3 receptor and its role in intracellular ligand processing in rat osteoblastic cells. J. Cell. Physiol. 177, 563-574.

Walling, H.W., Raggatt, L.J., Irvine, D.W., Barmina, O.Y., Toledano, J.E., Goldring, M.B., Hruska, K.A., Adkissson, H.D., Burdge, R.A., Gatt Jr., C.J., Harwood, D.A., Partridge, N.C., 2003. Impairment of the collagenase-3 endocytotic receptor system in cells from patients with osteoarthritis. Osteoarthritis Cartilage 11, 854-863.

Walsh, N., Larkin, A., Swan, N., Conlon, K., Dowling, P., McDermott, R., Clynes, M., 2011. RNAi knockdown of Hop (Hsp70/Hsp90 organising protein) decreases invasion via MMP-2 down regulation. Cancer Lett. 306, 180-189.

Wang, X., Yi, J., Lei, J., Pei, D., 1999a. Expression, purification, and characterization of recombinant mouse MT5-MMP protein products. FEBS Lett. 462, 261266.

Wang, Y., Johnson, A.R., Ye, Q.Z., Dyer, R.D., 1999b. Catalytic activities and substrate specificity of the human membrane type 4 matrix metalloproteinase catalytic domain. J. Biol. Chem. 274, 33043-33049.

Wang, X., Ma, D., Keski-Oja, J., Pei, D., 2004a. Co-recycling of MT1-MMP and MT3-MMP through the trans-Golgi network. Identification of DKV582 as a recycling signal. J. Biol. Chem. 279, 9331-9336.

Wang, Y., Middleton, F., Horton, J.A., Reichel, L., Farnum, C.E., Damron, T.A., 2004b. Microarray analysis of proliferative and hypertrophic growth plate zones identifies differentiation markers and signal pathways. Bone 35, 1273-1293. 
Wang, X., Liang, J., Koike, T., Sun, H., Ichikawa, T., Kitajima, S., Morimoto, M., Shikama, H., Watanabe, T., Sasaguri, Y., Fan, J., 2004c. Overexpression of human matrix metalloproteinase-12 enhances the development of inflammatory arthritis in transgenic rabbits. Am. J. Pathol. 165, $1375-1383$.

Wang, F.Q., So, J., Reierstad, S., Fishman, D.A., 2005. Matrilysin (MMP-7) promotes invasion of ovarian cancer cells by activation of progelatinase. Int. J. Cancer 114, 19-31.

Wang, F., Reierstad, S., Fishman, D.A., 2006. Matrilysin over-expression in MCF-7 cells enhances cellular invasiveness and pro-gelatinase activation. Cancer Lett. 236, 292-301.

Wang, X., Chow, F.L., Oka, T., Hao, L., Lopez-Campistrous, A., Kelly, S., Cooper, S., Odenbach, J., Finegan, B.A., Schulz, R., Kassiri, Z., Lopaschuk, G.D., FernandezPatron, C., 2009. Matrix metalloproteinase-7 and ADAM-12 (a disintegrin and metalloproteinase-12) define a signaling axis in agonist-induced hypertension and cardiac hypertrophy. Circulation 119, 2480-2489.

Wang, L., Wang, C.H., Jia, J.F., Ma, X.K., Li, Y., Zhu, H.B., Tang, H., Chen, Z.N., Zhu, P., 2010a. Contribution of cyclophilin A to the regulation of inflammatory processes in rheumatoid arthritis. J. Clin. Immunol. 30, 24-33.

Wang, Y., Ait-Oufella, H., Herbin, O., Bonnin, P., Ramkhelawon, B., Taleb, S., Huang, J., Offenstadt, G., Combadière, C., Renia, L., Johnson, J.L., Tharaux, P.L., Tedgui, A., Mallat, Z., 2010b. TGF-beta activity protects against inflammatory aortic aneurysm progression and complications in angiotensin II-infused mice. J. Clin. Invest. 120, 422-432.

Wang, Y., Hu, X., Xie, X., He, A., Liu, X., Wang, J.A., in press. Effects of mesenchymal stem cells on matrix metalloproteinase synthesis in cardiac fibroblasts. Exp. Biol. Med.

Ware, L.B., Matthay, M.A., 2000. The acute respiratory distress syndrome. N. Engl. J. Med. 342, 1334-1349.

Warner, R.L., Beltran, L., Younkin, E.M., Lewis, C.S., Weiss, S.J., Varani, J., Johnson, K.J., 2001a. Role of stromelysin 1 and gelatinase B in experimental acute lung injury. Am. J. Respir. Cell Mol. Biol. 24, 537-544.

Warner, R.L., Lewis, C.S., Beltran, L., Younkin, E.M., Varani, J., Johnson, K.J., 2001b. The role of metalloelastase in immune complex-induced acute lung injury. Am. J. Pathol. 158, 2139-2144.

Warner, R.L., Lukacs, N.W., Shapiro, S.D., Bhagarvathula, N., Nerusu, K.C., Varani, J., Johnson, K.J., 2004. Role of metalloelastase in a model of allergic lung responses induced by cockroach allergen. Am. J. Pathol. 165, 1921-1930.

Watson, A.M., Benton, A.S., Rose, M.C., Freishtat, R.J., 2010. Cigarette smoke alters tissue inhibitor of metalloproteinase 1 and matrix metalloproteinase 9 levels in the basolateral secretions of human asthmatic bronchial epithelium in vitro. J. Investig. Med. 58, 725-729.

Weaver, A., Gonçalves da Silva, A., Nuttall, R.K., Edwards, D.R., Shapiro, S.D., Rivest, S., Yong, V.W., 2005. An elevated matrix metalloproteinase (MMP) in an animal model of multiple sclerosis is protective by affecting Th1/Th2 polarization. FASEB J. 19, 1668-1670.

Wei, P.C., Tsai, C.H., Chiu, P.S., Lai, S.C., 2011. Matrix metalloproteinase-12 leads to elastin degradation in BALB/c mice with eosinophilic meningitis caused by Angiostrongylus cantonensis. Int. J. Parasitol. 41, 1175-1183.

Weingarten, H., Martin, R., Feder, J., 1985. Synthetic substrates of vertebrate collagenase. Biochemistry 24, 6730-6734.

Wells, J.E., Rice, T.K., Nuttall, R.K., Edwards, D.R., Zekki, H., Rivest, S., Yong, V.W., 2003. An adverse role for matrix metalloproteinase 12 after spinal cord injury in mice. J. Neurosci. 23, 10107-10115.

Wells, J.E.A., Biernaskie, J., Szymanska, A., Larsen, P.H., Yong, V.W., Corbett, D., 2005. Matrix metalloproteinase (MMP)-12 expression has a negative impact on sensorimotor function following intracerebral haemorrhage in mice. Eur. J. Neurosci. 21, 187-196.

Werb, Z., Chin, J.R., 1998. Extracellular matrix remodeling during morphogenesis. Ann. NY Acad. Sci. 857, 110-118.

Werner, S.R., Mescher, A.L., Neff, A.W., King, M.W., Chaturvedi, S., Duffin, K.L., Harty, M.W., Smith, R.C., 2007. Neural MMP-28 expression precedes myelination during development and peripheral nerve repair. Dev. Dyn. 236, 2852-2864.

Werner, S.R., Dotzlaf, J.E., Smith, R.C., 2008. MMP-28 as a regulator of myelination. BMC Neurosci. 9, 83.

Wernicke, D., Seyfert, C., Hinzmann, B., Gromnica-Ihle, E., 1996. Cloning of collagenase 3 from the synovial membrane and its expression in rheumatoid arthritis and osteoarthritis. J. Rheumatol. 23, 590-595.

Weskamp, G., Ford, J.W., Sturgill, J., Martin, S., Docherty, A.J., Swendeman, S., Broadway, N., Hartmann, D., Saftig, P., Umland, S., Sehara-Fujisawa, A., Black, R.A., Ludwig, A., Becherer, J.D., Conrad, D.H., Blobel, C.P., 2006. ADAM10 is a principal 'sheddase' of the low-affinity immunoglobulin E receptor CD23. Nat. Immunol. 7, 1293-1298.

Westhoff, C.S., Freudiger, D., Petrow, P., Seyfert, C., Zacher, J., Kriegsmann, J., Pap, T., Gay, S., Stiehl, P., Gromnica-Ihle, E., Wernicke, D., 1999. Characterization of collagenase 3 (matrix metalloproteinase 13) messenger RNA expression in the synovial membrane and synovial fibroblasts of patients with rheumatoid arthritis. Arthritis Rheum. 42, 1517-1527.

White, J.M., 2003. ADAMs: modulators of cell-cell and cell-matrix interactions. Curr. Opin. Cell Biol. 15, 598-606.

White, J.M., Bridges, L., DeSimone, D., Tomczuk, M., Wolfsberg, T., 2005. Introduction to the ADAM Family. In: Hooper, N.M., Lendeckel, U. (Eds.), The ADAM.

Whitelock, J.M., Murdoch, A.D., Iozzo, R.V., Underwood, P.A., 1996. The degradation of human endothelial cell-derived perlecan and release of bound basic fibroblast growth factor by stromelysin, collagenase, plasmin, and heparanases. J. Biol. Chem. 271, 10079-10086.

Widerlöv, E., Lindström, L.H., Besev, G., Manberg, P.J., Nemeroff, C.B., Breese, G.R., Kizer, J.S., Prange Jr., A.J., 1982. Subnormal CSF levels of neurotensin in a subgroup of schizophrenic patients: normalization after neuroleptic treatment. Am. J. Psychiatry 139, 1122-1126.

Wiegand, C., Schönfelder, U., Abel, M., Ruth, P., Kaatz, M., Hipler, U.C., 2010. Protease and pro-inflammatory cytokine concentrations are elevated in chronic compared to acute wounds and can be modulated by collagen type I in vitro. Arch. Dermatol. Res. 302, 419-428.

Wielockx, B., Lannoy, K., Shapiro, S.D., Itoh, T., Itohara, S., Vandekerckhove, J., Libert, C., 2001. Inhibition of matrix metalloproteinases blocks lethal hepatitis and apoptosis induced by tumor necrosis factor and allows safe antitumor therapy. Nat. Med. 7, 1202-1208.

Wildeboer, D., Naus, S., Amy Sang, Q.X., Bartsch, J.W., Pagenstecher, A., 2006. Metalloproteinase disintegrins ADAM8 and ADAM19 are highly regulated in human primary brain tumors and their expression levels and activities are associated with invasiveness. J. Neuropathol. Exp. Neurol. 65, 516-527.

Wilkins-Port, C.E., Ye, Q., Mazurkiewicz, J.E., Higgins, P.J., 2009. TGF-beta1 + EGF-initiated invasive potential in transformed human keratinocytes is coupled to a plasmin/MMP-10/MMP-1-dependent collagen remodeling axis: role for PAI-1. Cancer Res. 69, 4081-4091.

Williams, H., Johnson, J.L., Jackson, C.L., White, S.J., George, S.J., 2010. MMP-7 mediates cleavage of N-cadherin and promotes smooth muscle cell apoptosis. Cardiovasc. Res. 87, 137-146.

Wilson, C.L., Matrisian, L.M., 1996. Matrilysin: an epithelial matrix metalloproteinase with potentially novel functions. Int. J. Biochem. Cell Biol. 28, 123136.

Wilson, C.L., Ouellette, A.J., Satchell, D.P., Ayabe, T., Lopez-Boado, Y.S., Stratman, J.L., Hultgren, S.J., Matrisian, L.M., Parks, W.C., 1999. Regulation of intestinal alpha-defensin activation by the metalloproteinase matrilysin in innate host defense. Science 286, $113-117$.

Wilson, W.R., Schwalbe, E.C., Jones, J.L., Bell, P.R., Thompson, M.M., 2005. Matrix metalloproteinase 8 (neutrophil collagenase) in the pathogenesis of abdominal aortic aneurysm. Br. J. Surg. 92, 828-833.

Winkler, M.K., Foldes, J.K., Bunn, R.C., Fowlkes, J.L., 2003. Implications for matrix metalloproteinases as modulators of pediatric lung disease. Am. J. Physiol. Lung Cell. Mol. Physiol. 284, L557-L565.

Witty, J.P., Wright, J.H., Matrisian, L.M., 1995. Matrix metalloproteinases are expressed during ductal and alveolar mammary morphogenesis, and misregulation of stromelysin-1 in transgenic mice induces unscheduled alveolar development. Mol. Biol. Cell 6, 1287-1303.

Woessner, J.F., Nagase, H., 2000. Matrix Metalloproteinases and TIMPs. Oxford University Press, New York.

Wolf, C., Chenard, M.P., Durand de Grossouvre, P., Bellocq, J.P., Chambon, P., Basset, P., 1992. Breast-cancer-associated stromelysin-3 gene is expressed in basal cell carcinoma and during cutaneous wound healing. J. Invest. Dermatol. 99, 870-872.

Wolfman, N.M., McPherron, A.C., Pappano, W.N., Davies, M.V., Song, K., Tomkinson, K.N., Wright, J.F., Zhao, L., Sebald, S.M., Greenspan, D.S., Lee, S.J., 2003. Activation of latent myostatin by the BMP-1/tolloid family of metalloproteinases. Proc. Natl. Acad. Sci. USA $100,15842-15846$.

Wright, R.W., Allen, T., El-Zawawy, H.B., Brodt, M.D., Silva, M.J., Gill, C.S., Sandell, L.J., 2006. Medial collateral ligament healing in macrophage metalloelastase (MMP-12)-deficient mice. J. Orthop. Res. 24, 2106-2113. 
Wu, E., Croucher, P.I., Mckie, N., 1997. Expression of members of the novel membrane linked metalloproteinase family ADAM in cells derived from a range of haematological malignancies. Biochem. Biophys. Res. Commun. 235, 437-442.

Wu, J., Ru, N.Y., Zhang, Y., Li, Y., Wei, D., Ren, Z., Huang, X.F., Chen, Z.N., Bian, H., 2011a. HAb18G/CD147 promotes epithelial-mesenchymal transition through TGF- $\beta$ signaling and is transcriptionally regulated by Slug. Oncogene 30, 4410-4427.

Wu, T., Li, Y., Liu, X., Lu, J., He, X., Wang, Q., Li, J., Du, X., 2011b. Identification of high-risk stage II and stage III colorectal cancer by analysis of MMP-21 expression. J. Surg. Oncol. 104, 787-791.

Wysocki, A.B., Staiano-Coico, L., Grinnell, F., 1993. Wound fluid from chronic leg ulcers contains elevated levels of metalloproteinases MMP-2 and MMP-9. J. Invest. Dermatol. 101, 64-68.

Xia, H., Qi, Y., Ng, S.S., Chen, X., Li, D., Chen, S., Ge, R., Jiang, S., Li, G., Chen, Y., He, M.L., Kung, H.F., Lai, L., Lin, M.C., 2009. MicroRNA-146b inhibits glioma cell migration and invasion by targeting MMPs. Brain Res. 1269, 158-165.

Xiong, W., Knispel, R., MacTaggart, J., Greiner, T.C., Weiss, S.J., Baxter, B.T., 2009. Membrane-type 1 matrix metalloproteinase regulates macrophagedependent elastolytic activity and aneurysm formation in vivo. J. Biol. Chem. 284, 1765-1771.

Xu, D., Emoto, N., Giaid, A., Slaughter, C., Kaw, S., de Wit, D., Yanagisawa, M., 1994. ECE-1: a membrane-bound metalloprotease that catalyzes the proteolytic activation of big endothelin-1. Cell 78, 473-485.

Xu, J., Rodriguez, D., Petieclere, E., Kim, J.J., Hangai, M., Moon, Y.S., Davis, G.E., Brooks, P.C., 2001. Proteolytic exposure of a cryptic site within collagen type IV is required for angiogenesis and tumor growth in vivo. J. Cell Biol. 154, 1069-1080.

Xu, X., Wang, Y., Lauer-Fields, J.L., Fields, G.B., Steffensen, B., 2004a. Contributions of the MMP-2 collagen binding domain to gelatin cleavage. Substrate binding via the collagen binding domain is required for hydrolysis of gelatin but not short peptides. Matrix Biol. 23, 171-181.

Xu, E., Lai, M., Lv, B., Xing, X., Huang, Q., Xia, X., 2004b. A single nucleotide polymorphism in the matrix metalloproteinase-2 promoter is associated with colorectal cancer. Biochem. Biophys. Res. Commun. 324, 999-1003.

Xu, X., Chen, Z., Wang, Y., Bonewald, L., Steffensen, B., 2007. Inhibition of MMP-2 gelatinolysis by targeting exodomain-substrate interactions. Biochem. J. 406, 147-155.

Xu, D., McKee, C.M., Cao, Y., Ding, Y., Kessler, B.M., Muschel, R.J., 2010. Matrix metalloproteinase-9 regulates tumor cell invasion through cleavage of protease nexin-1. Cancer Res. 70, 6988-6998.

Yamada, S., Wang, K.Y., Tanimoto, A., Fan, J., Shimajiri, S., Kitajima, S., Morimoto, M., Tsutsui, M., Watanabe, T., Yasumoto, K., Sasaguri, Y., 2008. Matrix metalloproteinase 12 accelerates the initiation of atherosclerosis and stimulates the progression of fatty streaks to fibrous plaques in transgenic rabbits. Am. J. Pathol. 172, 1419-1429.

Yamamoto, H., Adachi, Y., Itoh, F., Iku, S., Matsuno, K., Kusano, M., Arimura, Y., Endo, T., Hinoda, Y., Hosokawa, M., Imai, K., 1999. Association of matrilysin expression with recurrence and poor prognosis in human esophageal squamous cell carcinoma. Cancer Res. 59, 3313-3316.

Yamamoto, H., Itoh, F., Iku, S., Adachi, Y., Fukushima, H., Sasaki, S., Mukaiya, M., Hirata, K., Imai, K., 2001. Expression of matrix metalloproteinases and tissue inhibitors of metalloproteinases in human pancreatic adenocarcinomas: clinicopathologic and prognostic significance of matrilysin expression. J. Clin. Oncol. 19, 1118-1127.

Yamamoto, D., Takai, S., 2009. Pharmacological implications of MMP-9 inhibition by ACE inhibitors. Curr. Med. Chem. 16, $1349-1354$.

Yamanaka, H., Makino, K., Takizawa, M., Nakamura, H., Fujimoto, N., Moriya, H., Nemori, R., Sato, H., Seiki, M., Okada, Y., 2000. Expression and tissue localization of membrane-types 1, 2, and 3 matrix metalloproteinases in rheumatoid synovium. Lab. Invest. 80, 677-687.

Yan, P., Hu, X., Song, H., Yin, K., Bateman, R.J., Cirrito, J.R., Xiao, Q., Hsu, F.F., Turk, J.W., Xu, J., Hsu, C.Y., Holtzman, D.M., Lee, J.M., 2006. Matrix metalloproteinase-9 degrades amyloid-beta fibrils in vitro and compact plaques in situ. J. Biol. Chem. 281, 24566-24574.

Yana, I., Weiss, S.J., 2000. Regulation of membrane type-1 matrix metalloproteinase activation by proprotein convertases. Mol. Biol. Cell 11, 2387-2401.

Yana, I., Seiki, M., 2002. MT-MMPs play pivotal roles in cancer dissemination. Clin. Exp. Metastasis 19, 209-215.

Yang, Y., Estrada, E.Y., Thompson, J.F., Liu, W., Rosenberg, G.A., 2007a. Matrix metalloproteinase-mediated disruption of tight junction proteins in cerebral vessels is reversed by synthetic matrix metalloproteinase inhibitor in focal ischemia in rat. J. Cereb. Blood Flow Metab. $27,697-709$.

Yang, X., Dong, Y., Zhao, J., Sun, H., Deng, Y., Fan, J., Yan, Q., 2007b. Increased expression of human macrophage metalloelastase (MMP-12) is associated with the invasion of endometrial adenocarcinoma. Pathol. Res. Pract. 203, 499-505.

Yang, Y., Lu, N., Zhou, J., Chen, Z.N., Zhu, P., 2008. Cyclophilin A up-regulates MMP-9 expression and adhesion of monocytes/macrophages via CD147 signalling pathway in rheumatoid arthritis. Rheumatology (Oxford) 47, 1299-1310.

Yavari, R., Adida, C., Bray-Ward, P., Brines, M., Xu, T., 1998. Human metalloprotease-disintegrin Kuzbanian regulates sympathoadrenal cell fate in development and neoplasia. Hum. Mol. Genet. 7, 1161-1167.

Ye, S., Eriksson, P., Hamsten, A., Kurkinen, M., Humphries, S.E., Henney, A.M., 1996. Progression of coronary atherosclerosis is associated with a common genetic variant of the human stromelysin-1 promoter which results in reduced gene expression. J. Biol. Chem. 271, 13055-13060.

Ye, S., 2006. Influence of matrix metalloproteinase genotype on cardiovascular disease susceptibility and outcome. Cardiovasc. Res. 69, 636-645

Ye, S., Patodi, N., Walker-Bone, K., Reading, I., Cooper, C., Dennison, E., 2007. Variation in the matrix metalloproteinase-3, -7, -12 and -13 genes is associated with functional status in rheumatoid arthritis. Int. J. Immunogenet. 34, 81-85.

Yen, J.H., Kong, W., Ganea, D., 2010. IFN-beta inhibits dendritic cell migration through STAT-1-mediated transcriptional suppression of CCR7 and matrix metalloproteinase 9. J. Immunol. 184, 3478-3486.

Yoon, S., Kuivaniemi, H., Gatalica, Z., Olson, J.M., Buttice, G., Ye, S., Norris, B.A., Malcom, G.T., Strong, J.P., Tromp, G., 2002. MMP13 promoter polymorphism is associated with atherosclerosis in the abdominal aorta of young black males. Matrix Biol. 21, 487-498.

Yoshihara, Y., Nakamura, H., Obata, K., Yamada, H., Hayakawa, T., Fujikawa, K., Okada, Y., 2000. Matrix metalloproteinases and tissue inhibitors of metalloproteinases in synovial fluids from patients with rheumatoid arthritis or osteoarthritis. Ann. Rheum. Dis. 59, 455-461.

Yoshizaki, T., Sato, H., Maruyama, Y., Murono, S., Furukawa, M., Park, C.S., Seiki, M., 1997. Increased expression of membrane type 1-matrix metalloproteinase in head and neck carcinoma. Cancer 79, 139-144.

Yoshizaki, T., Maruyama, Y., Sato, H., Furukawa, M., 2001. Expression of tissue inhibitor of matrix metalloproteinase-2 correlates with activation of matrix metalloproteinase-2 and predicts poor prognosis in tongue squamous cell carcinoma. Int. J. Cancer 95, 44-50.

Young, S.G., Fong, L.G., Michaelis, S., 2005. Prelamin A, Zmpste24, misshapen cell nuclei, and progeria - new evidence suggesting that protein farnesylation could be important for disease pathogenesis. J. Lipid Res. 46, 2531-2558.

Young, S.G., Meta, M., Yang, S.H., Fong, L.G., 2006. Prelamin A farnesylation and progeroid syndromes. J. Biol. Chem. 281, $39741-39745$.

Yu, Q., Stamenkovic, I., 2000. Cell surface-localized matrix metalloproteinase-9 proteolytically activates TGF-beta and promotes tumor invasion and angiogenesis. Genes Dev. 14, 163-176.

Yu, W.H., Woessner, J.F.J., McNeish, J.D., Stamenkovic, I., 2002. CD44 anchors the assembly of matrilysin/MMP-7 with heparin-binding epidermal growth factor precursor and ErbB4 and regulates female reproductive organ remodeling. Genes Dev. 16, 307-323.

Zeng, B., Prasan, A., Fung, K.C., Solanki, V., Bruce, D., Freedman, S.B., Brieger, D., 2005. Elevated circulating levels of matrix metalloproteinase-9 and -2 in patients with symptomatic coronary artery disease. Intern. Med. J. 35, 331-335.

Zhang, X., Gonnella, N.C., Koehn, J., Pathak, N., Ganu, V., Melton, R., Parker, D., Hu, S.I., Nam, K.Y., 2000. Solution structure of the catalytic domain of human collagenase-3 (MMP-13) complexed to a potent non-peptidic sulfonamide inhibitor: binding comparison with stromelysin-1 and collagenase-1. J. Mol. Biol. 301, 513-524.

Zhang, J., Jin, X., Fang, S., Wang, R., Li, Y., Wang, N., Guo, W., Wang, Y., Wen, D., Wei, L., Dong, Z., Kuang, G., 2005a. The functional polymorphism in the matrix metalloproteinase-7 promoter increases susceptibility to esophageal squamous cell carcinoma, gastric cardiac adenocarcinoma and non-small cell lung carcinoma. Carcinogenesis 26, 1748-1753.

Zhang, J., Sarkar, S., Yong, V.W., 2005b. The chemokine stromal cell derived factor-1 (CXCL12) promotes glioma invasiveness through MT2-matrix metalloproteinase. Carcinogenesis 26, 2069-2077. 
Zhao, H., Bernardo, M.M., Osenkowski, P., Sohail, A., Pei, D., Nagase, H., Kashiwagi, M., Soloway, P.D., DeClerck, Y.A., Fridman, R., 2004. Differential inhibition of membrane type 3 (MT3)-matrix metalloproteinase (MMP) and MT1-MMP by tissue inhibitor of metalloproteinase (TIMP)-2 and TIMP-3 regulates pro-MMP-2 activation. J. Biol. Chem. 279, 8592-8601.

Zhao, X., Benveniste, E.N., 2008. Transcriptional activation of human matrix metalloproteinase-9 gene expression by multiple co-activators. J. Mol. Biol. 383, $945-956$.

Zhao, H., Sohail, A., Sun, Q., Shi, Q., Kim, S., Mobashery, S., Fridman, R., 2008. Identification and role of the homodimerization interface of the glycosylphosphatidylinositol-anchored membrane type 6 matrix metalloproteinase (MMP-25). J. Biol. Chem. 283, 35023-35032.

Zhao, L., Lin, S., Bales, K.R., Gelfanova, V., Koger, D., Delong, C., Hale, J., Liu, F., Hunter, J.M., Paul, S.M., 2009. Macrophage-mediated degradation of betaamyloid via an apolipoprotein E isoform-dependent mechanism. J. Neurosci. 29, 3603-3612.

Zheng, L., Lam, W.K., Tipoe, G.L., Shum, I.H., Yan, C., Leung, R., Sun, J., Ooi, G.C., Tsang, K.W., 2002. Overexpression of matrix metalloproteinase-8 and -9 in bronchiectasic airways in vivo. Eur. Respir. J. 20, 170-176.

Zhong, W.D., Han, Z.D., He, H.C., Bi, X.C., Dai, Q.S., Zhu, G., Ye, Y.K., Liang, Y.X., Qin, W.J., Zhang, Z., Zeng, G.H., Chen, Z.N., 2008. CD147, MMP-1, MMP-2 and MMP-9 protein expression as significant prognostic factors in human prostate cancer. Oncology 75, $230-236$.

Zhou, Z., Apte, S.S., Soininen, R., Cao, R., Baaklini, G.Y., Rauser, R.W., Wang, J., Cao, Y., Tryggvason, K., 2000. Impaired endochondral ossification and angiogenesis in mice deficient in membrane-type matrix metalloproteinase I. Proc. Natl. Acad. Sci. USA 97, $4052-4057$.

Zhou, H., Wong, Y.F., Wang, J., Cai, X., Liu, L., 2008. Sinomenine ameliorates arthritis via MMPs, TIMPs, and cytokines in rats. Biochem. Biophys. Res. Commun. 376, 352-357.

Zhu, P., Lu, N., Shi, Z.G., Zhou, J., Wu, Z.B., Yang, Y., Ding, J., Chen, Z.N., 2006. CD147 overexpression on synoviocytes in rheumatoid arthritis enhances matrix metalloproteinase production and invasiveness of synoviocytes. Arthritis Res. Ther. 8, R44.

Zijlstra, A., Seandel, M., Kupriyanova, T.A., Partridge, J.J., Madsen, M.A., Hahn-Dantona, E.A., Quigley, J.P., Deryugina, E.I., 2006. Proangiogenic role of neutrophil-like inflammatory heterophils during neovascularization induced by growth factors and human tumor cells. Blood 107, $317-327$.

Zitta, K., Brandt, B., Wuensch, A., Meybohm, P., Bein, B., Steinfath, M., Scholz, J., Albrecht, M., 2010. Interleukin-1beta regulates cell proliferation and activity of extracellular matrix remodelling enzymes in cultured primary pig heart cells. Biochem. Biophys. Res. Commun. 399, 542-547.

Zucker, S., Pei, D., Cao, J., Lopez-Otin, C., 2003. Membrane type-matrix metalloproteinases (MT-MMP). Curr. Top. Dev. Biol. 54, 1-74.

Zuo, F., Kaminski, N., Eugui, E., Allard, J., Yakhini, Z., Ben-Dor, A., Lollini, L., Morris, D., Kim, Y., De Lustro, B., Sheppard, D., Pardo, A., Selman, M., Heller, R.A., 2002. Gene expression analysis reveals matrilysin as a key regulator of pulmonary fibrosis in mice and humans. Proc. Natl. Acad. Sci. USA 99, 62926297. 